Universidad

Politécnica

de Cartagena
Universidad | Campus

Politécnica de Excelencia

de Cartagena Internacional

\title{
Contribution to the Design of Nanodevices and Communication \\ Protocols for Nanonetworks in the $\mathrm{THz}$
}

band

P

h

D

Department of Information and Communication Technologies

$\mathrm{T}$
$\mathrm{H}$
$\mathrm{E}$
$\mathrm{S}$
$\mathrm{I}$
$\mathrm{S}$

Sebastián Cánovas Carrasco

Directors:

Joan García Haro

Author:

Antonio Javier García Sánchez

Cartagena 2020 

Universidad

Politécnica

de Cartagena

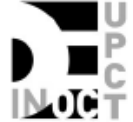

DT-16

\section{CONFORMIDAD DE SOLICITUD DEAUTORIZACIÓN DE DEPÓSITO DE}

TESIS DOCTORAL POR EL/LA DIRECTOR/A DE LA TESIS

D. Joan García Haro, Director de la Tesis doctoral "Contribution to the Design of Nanodevices and Communication Protocols for Nanonetworks in the THz band",

\section{INFORMA:}

Que la referida Tesis Doctoral, ha sido realizada por D. Sebastián Cánovas Carrasco, dentro del Programa de Doctorado "Tecnologías de la Información y las Comunicaciones", dando mi conformidad para que sea presentada ante el Comité de Dirección de la Escuela Internacional de Doctorado para ser autorizado su depósito.

\Informe positivo sobre el plan de investigación y documento de actividades del doctorando/a emitido por el Director/ Tutor (RAPI)

La rama de conocimiento en la que esta tesis ha sido desarrollada es:

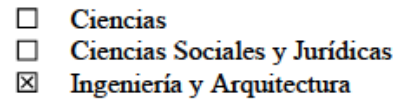

En Cartagena, a 4 de septiembre de 2020

EL DIRECTOR DE LA TESIS

JUAN|

GARCIA

Fdo.

HARO

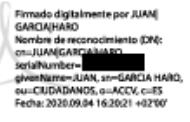



Universidad

Politécnica

de Cartagena

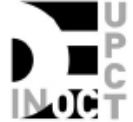

DT-16

\section{CONFORMIDAD DE SOLICITUD DEAUTORIZACIÓN DE DEPÓSITO DE}

\section{TESIS DOCTORAL POR EL/LA DIRECTOR/A DE LA TESIS}

D. Antonio Javier García Sánchez, Co-director de la Tesis doctoral "Contribution to the Design of Nanodevices and Communication Protocols for Nanonetworks in the THz band",

\section{INFORMA:}

Que la referida Tesis Doctoral, ha sido realizada por D. Sebastián Cánovas Carrasco, dentro del Programa de Doctorado "Tecnologías de la Información y las Comunicaciones", dando mi conformidad para que sea presentada ante el Comité de Dirección de la Escuela Internacional de Doctorado para ser autorizado su depósito.

\Informe positivo sobre el plan de investigación y documento de actividades del doctorando/a emitido por el Director/ Tutor (RAPI).

La rama de conocimiento en la que esta tesis ha sido desarrollada es:

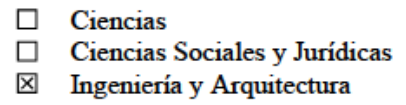

En Cartagena, a 4 de septiembre de 2020

EL CO-DIRECTOR DE LA TESIS

ANTONIO Firmado digitalmente por

JAVIER|GARCIA| $/$ ANTONIO JAMER/GARCAA]

SANCHEZ Fecha:

Fdo: 

Universidad

Politécnica

de Cartagena

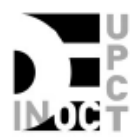

DT-17

\section{CONFORMIDAD DE DEPÓSITO DE TESIS DOCTORAL}

\section{POR LA COMISIÓN ACADÉMICA DEL PROGRAMA}

D. Jorge Larrey Ruiz, Presidente de la Comisión Académica del Programa de Doctorado Tecnologías de la Información y las Comunicaciones,

\section{INFORMA:}

Que la Tesis Doctoral titulada, "Contribution to the Design of Nanodevices and Communication Protocols for Nanonetworks in the THz band", ha sido realizada, dentro del mencionado Programa de Doctorado, por D. Sebastián Cánovas Carrasco, bajo la dirección y supervisión del Dr Joan García Haro.

En reunión de la Comisión Académica, visto que en la misma se acreditan los indicios de calidad correspondientes y la autorización del Director/a de la misma, se acordó dar la conformidad, con la finalidad de que sea autorizado su depósito por el Comité de Dirección de la Escuela Internacional de Doctorado.

囚 Evaluación positiva del plan de investigación y documento de actividades por el Presidente de la Comisión Académica del programa (RAPI)

La Rama de conocimiento por la que esta tesis ha sido desarrollada es:

\section{Ciencias}

Ciencias Sociales y Jurídicas

囚 Ingeniería y Arquitectura

En Cartagena, a 4 de septiembre de 2020

EL PRESIDENTE DE LA COMISIÓN ACADÉMICA

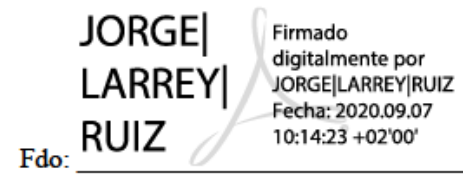





\section{Universidad}

Politécnica

de Cartagena

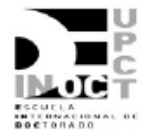

Sr. D. Sebastián Cánovas Carrasco

Visto el informe favorable del Director de Tesis y el № Bo de la Comisión Académica del Programa de Doctorado "Tecnologías de la Información y las Comunicaciones" para la presentación de la Tesis Doctoral titulada: "Contribution to the Design of Nanodevices and Communication Protocols for Nanonetworks in the THz band" solicitada por D. SEBASTIÁN CÁNOVAS CARRASCO, el Comité de Dirección de la Escuela Internacional de Doctorado de la Universidad Politécnica de Cartagena, en reunión celebrada el 29 de septiembre de 2020, considerando lo dispuesto en el artículo 23 del Reglamento de Estudios Oficiales de Doctorado de la UPCT, aprobado en Consejo de Gobierno el 17 de diciembre de 2015,

\section{ACUERDA}

Autorizar la presentación de la Tesis Doctoral a D. Sebastián Cánovas Carrasco en la modalidad de "compendio de publicaciones".

Contra el presente acuerdo, que no agota la vía administrativa, podrá formular recurso de alzada ante el Sr. Rector-Magnífico de la Universidad Politécnica de Cartagena, en el plazo de un mes a partir de la notificación de la presente.

Cartagena, 29 de septiembre de 2020

EL DIRECTOR DE LA ESCUELA

INTERNACIONAL DE DOCTORADO

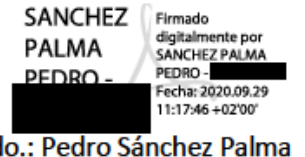

Fdo.: Pedro Sánchez Palma 

This thesis is presented as a compilation of the following articles:

1. S. Canovas-Carrasco, A.-J. Garcia-Sanchez, F. Garcia-Sanchez, and J. Garcia-Haro, "Conceptual design of a nano-networking device," Sensors, vol. 16, no. 12, p. 2104, Dec. 2016. [1]

2. S. Canovas-Carrasco, A.-J. Garcia-Sanchez, and J. Garcia-Haro, "The IEEE 1906.1 Standard: Nanocommunications as a new source of data," 2017 ITU Kaleidoscope: Challenges for a Data-Driven Society (ITU K), Nanjing, 2017, pp. 1-7. 2

3. S. Canovas-Carrasco, A.-J. Garcia-Sanchez, and J. Garcia-Haro, "A nanoscale communication network scheme and energy model for a human hand scenario," Nano Communication Networks, vol. 15, pp. 17-27, Mar. 2018. 3

4. S. Canovas-Carrasco, A.-J. Garcia-Sanchez, and J. Garcia-Haro, "On the Nature of Energy-Feasible Wireless Nanosensor Networks," Sensors, vol. 18, no. 5, p. 1356, Apr. 2018. 4

5. S. Canovas-Carrasco, A.-J. Garcia-Sanchez, and J. Garcia-Haro, "The IEEE 1906.1 standard: Some guidelines for strengthening future normalization in electromagnetic nanocommunications," IEEE Commun. Stand. Mag., vol. 2, no. 4, pp. 26-32, Dec. 2018. 5]

6. S. Canovas-Carrasco, R. M. Sandoval, A.-J. Garcia-Sanchez, and J. Garcia-Haro, "Optimal Transmission Policy Derivation for IoNT FlowGuided Nano-Sensor Networks," IEEE Internet of Things Journal, vol. 6, no. 2, pp. 2288-2298, Apr. 2019.6

7. R. Asorey-Cacheda, S. Canovas-Carrasco, A.-J. Garcia-Sanchez, J. Garcia-Haro, "An Analytical Approach to Flow-Guided Nanocommunication Networks," Sensors, vol. 20, no. 5, p. 1332, Feb. 2020. 7

\section{International mention}

Research stay at University of California, Davis (Davis, USA). From 01/09/2019 to 01/12/2019 (3 months). 



\section{Agradecimientos}

A mis tutores, Antonio y Joan, por su trabajo durante estos 4 años y guiarme hacia un campo al que podía aportar nuevas nociones.

A mis compañeros de laboratorio, por compartir conmigo café e inquietudes.

A mis padres, que me han apoyado hasta el final en cada una de mis decisiones académicas.

A mi hermano, que, como yo, no abandona su condición de ingeniero a ninguna hora del día.

A Marina, por mostrarme su cariño y apoyo incondicional durante cada día de esta etapa. 



\begin{abstract}
Electromagnetic (EM) nano-communications is a new paradigm encompassing all concerns related to the exchange of information among devices operating in the Terahertz band (0.1-10 THz). A nano-network infrastructure consisting of a high number of nano-devices should be designed to ensure robust, reliable, and coordinated data transmission. This will enable a plethora of forthcoming applications and services in different research fields, such as medicine, environmental science, on-chip communications, or software-defined materials, which will lead to outstanding advances.

Nevertheless, EM nano-communications is a recent paradigm that lacks the maturity of other well-established communication fields, so the presented thesis contributes to this maturation process by researching both the hardware and the communication mechanisms upon which THz-based nano-networks could operate. As a Thesis by compilation, the 7 articles published during this Ph.D. program are presented and commented. Each of such articles looks into different key aspects that help closing the gap between theory and practice in $\mathrm{THz}$-based nano-communications.

Thus, in the first two chapters of this document, the reader will be introduced to the context and objectives of the thesis. In particular, the second chapter is divided into two subsections: general objectives, which are set into the global background; and specific objectives, grouped by publication. In the next seven chapters, the seven corresponding articles are presented and commented. For each of them, a brief summary, the methodology followed, an overview of the results and contributions, and final conclusions are included. Finally, in the two last chapters, the final conclusions and future lines of this thesis are discussed.
\end{abstract}





\section{Contents}

$\begin{array}{ll}\text { Contents } & 1\end{array}$

I Introduction, Context, and Objectives of this thesis 5

\begin{tabular}{llll}
\hline & Introduction and context & 7
\end{tabular}

1.1 Nano-device hardware . . . . . . . . . . . . . . . . . . . 9

1.2 Characterization of the communication channel in biological sce-

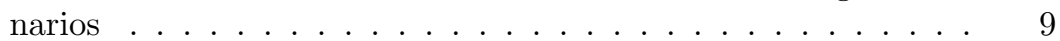

1.3 EM nano-communication techniques and nano-network architectures ........................ 10

$\begin{array}{lll}2 & \text { Objectives } & 13\end{array}$

2.1 General objectives $\ldots \ldots \ldots \ldots \ldots \ldots \ldots$

2.2 Specific objectives $\ldots \ldots \ldots \ldots \ldots \ldots$

\begin{tabular}{ll}
\hline II Original Articles & 19
\end{tabular}

1 Conceptual Design of a Nano-Networking Device 21

2 The IEEE 1906.1 standard: Nanocommunications as a new $\begin{array}{ll}\text { source of data } & 49\end{array}$

3 A nanoscale communication network scheme and energy model $\begin{array}{ll}\text { for a human hand scenario } & 57\end{array}$

4 On the nature of energy-feasible wireless nanosensor networks 69

5 The IEEE 1906.1 standard: Some guidelines for strengthening future normalization in electromagnetic nanocommunications

6 Optimal Transmission Policy Derivation for IoNT Flow-Guided Nano-Sensor Networks 
7 An Analytical Approach to Flow-Guided Nanocommunica$\begin{array}{ll}\text { tion Networks } & 103\end{array}$

\begin{tabular}{ll}
\hline III Summary of Articles & 127
\end{tabular}

1 Conceptual Design of a Nano-Networking Device 129

1.1 Brief summary $\ldots \ldots \ldots \ldots \ldots \ldots$

1.2 Methodology and tools . . . . . . . . . . . . . . . . . . . . 129

1.3 Results and Contributions . . . . . . . . . . . . . . . . 130

1.4 Conclusions . . . . . . . . . . . . . . . . . . . . . . . 130

2 The IEEE 1906.1 Standard: Nanocommunications as a new source of data 133

2.1 Brief summary . . . . . . . . . . . . . . . . . . . . . 133

2.2 Methodology and tools . . . . . . . . . . . . . . . . . . . 133

2.3 Results and Contributions . . . . . . . . . . . . . . . . . . 134

2.4 Conclusions . . . . . . . . . . . . . . . . . . . . . . . . 135

3 A nanoscale communication network scheme and energy model $\begin{array}{ll}\text { for a human hand scenario } & 137\end{array}$

3.1 Brief summary . . . . . . . . . . . . . . . . . . . . 137

3.2 Methodology and tools . . . . . . . . . . . . . . . . . . . . . 138

3.3 Results and Contributions . . . . . . . . . . . . . . . . . . . . 138

3.4 Conclusions . . . . . . . . . . . . . . . . . . . . . . . . . . . 139

4 On the Nature of Energy-Feasible Wireless Nanosensor Networks 141

4.1 Brief summary . . . . . . . . . . . . . . . . . . . . . . . 141

$4.2 \quad$ Methodology and tools . . . . . . . . . . . . . . . . . . . 142

4.3 Results and Contributions . . . . . . . . . . . . . . . . . . . . 142

4.4 Conclusions . . . . . . . . . . . . . . . . . . . . . . . . . . . 143

5 The IEEE 1906.1 standard: Some guidelines for strengthening future normalization in electromagnetic nanocommunications 145

5.1 Brief summary . . . . . . . . . . . . . . . . . . . . . . 145

5.2 Methodology and tools . . . . . . . . . . . . . . . . . 146

5.3 Results and Contributions . . . . . . . . . . . . . . . . 146

5.4 Conclusions . . . . . . . . . . . . . . . . . . . . . . . . 147

6 Optimal Transmission Policy Derivation for IoNT Flow-Guided Nano-Sensor Networks 149

6.1 Brief summary . . . . . . . . . . . . . . . . . . . . . . . 149

6.2 Methodology and tools . . . . . . . . . . . . . . . . . . 150

6.3 Results and Contributions . . . . . . . . . . . . . . . . . . . . 151

6.4 Conclusions . . . . . . . . . . . . . . . . . . . . . . . . . 152 
7 An Analytical Approach to Flow-Guided Nanocommunication Networks 153

7.1 Brief summary . . . . . . . . . . . . . . . . . . . . . . 153

7.2 Methodology and tools . . . . . . . . . . . . . . . . . 154

7.3 Results and Contributions . . . . . . . . . . . . . . . . 155

7.4 Conclusions . . . . . . . . . . . . . . . . . . . . . 157

IV Conclusions and Future Lines 159

\begin{tabular}{lll}
\hline 1 & Conclusions & 161
\end{tabular}

$\begin{array}{lll}2 & \text { Future Lines } & 165\end{array}$

\begin{tabular}{ll}
\hline V Appendices & 167 \\
\hline
\end{tabular}

\begin{tabular}{ll}
\hline VI References & 171
\end{tabular}

\begin{tabular}{ll}
\hline Bibliography & 173
\end{tabular} 



\section{Part I}

\section{Introduction, Context, and Objectives of this thesis}





\section{Chapter 1}

\section{Introduction and context}

In the so-called Internet of Things (IoT), small devices integrated into common objects are able to capture information from our environment (e.g. temperature, humidity, movement, etc.), and, if necessary, process, and analyze it. All these devices are part of a network infrastructure that joins the physical and virtual worlds, being able to interact between them autonomously. The IoT, as we know it, finds many applications in fields as wide as home automation, health care, smart cities and buildings, real-time monitoring of industrial processes, or smart transportation of people and goods, among others. Currently, one of the enabling technologies of IoT is known as Wireless Sensor Networks (WSN), in which many devices with limited energy consumption and low cost, connected between them, are able to monitor, collect, process and store information from the environment, and then make it available to the end-user. Applying this paradigm and due to the incessant technological innovations, new devices of increasingly reduced dimensions are being developed with the objective of offering novel applications and enabling a massive integration in the environment. However, aspects such as storage and processing of captured information, wireless communication coverage, or energy consumption, are much more problematic than those deployed in traditional WSN. Thus, when the scale of these new devices is in the order of micrometers or even below 8 and the terahertz $(\mathrm{THz})$ band $(0.1-10 \mathrm{THz})$ is employed, a new communication paradigm emerges which implies, on the one hand, one of the drifts of the well-known WSN and, on the other hand, an unprecedented advance within the IoT. This paradigm is the so-called electromagnetic (EM) nano-communications 9 11], which is the subject of study of this thesis.

Nanotechnology is a field of science focused on handling, controlling, and working with matter at a molecular and even atomic scale, providing the scientific community with an increasingly wide range of possibilities. Working at this scale, novel materials have been developed in the last few years that present properties that had not been observed at larger scales, creating the starting point for research on new devices and, consequently, the development of new applications. The family of two-dimensional materials, with graphene 
as the greatest exponent, is a clear example that, in a relatively short period, the foundations for developing a new generation of nano-devices can be laid down [12,13, ranging from nano-sensors 14,15,20], which play a fundamental role, for example, in new techniques for detecting diseases, to nano-actuators 16. 17], which may be able to administer medicines with higher precision than current techniques 18, 19. As an example of its potential, it has been demonstrated that a nano-sensor can detect chemical compounds in extremely low concentrations, which makes it possible to find cancer biomarkers at very early stages 20. It can be observed that the integration of this type of nano-sensor in a nano-device with computing and communication capabilities would represent an unquestionable advance in the field of medicine. Despite the wide range of new devices at the nanoscale, these have clear limitations, which go far beyond those of current WSN devices. With the development of nanotechnology, nano-devices may be able to perform very simple monitoring, processing, and even actuation tasks. Besides, they can incorporate a communication system that allows communication with other nodes in the network, which would give the possibility of building autonomous collaborative nano-networks that would perform more complex tasks and allow the implementation of nano-devices in scenarios hitherto inconceivable. Thus, to overcome the main challenges derived from the miniaturization of nano-devices, it becomes necessary to execute most of their tasks in collaboration with other nano-devices. For this reason, the development of protocols and communication techniques adapted to the characteristics of nano-devices is gathering increasing attention in the scientific community.

In this context, two main alternatives for communicating at the nanoscale have been envisaged so far, namely, molecular and EM nano-communications. On the one hand, molecular communication relies on the transmission and reception of information encoded in organic molecules [19, 21, 22. Molecular transceivers are designed to facilitate their integration into nanodevices due to their extremely small size and limited domain of operation. These transceivers can react when receiving certain molecules and release others (as a response to stimulation or after executing some process). On the other hand, EM communication is based on the use of EM waves for the communication between two nanodevices, in line with traditional WSN. This type of nanocommunication is based on nano-components that allow the radiation of EM waves at $\mathrm{THz}$ frequencies with antennas in the order of micrometers in length 13,23 26]. As mentioned above, this thesis is focused on this latter type of nano-communication since, from our point of view, it is more versatile due to its suitability to different propagation mediums (e.g., in-body, free space, on-chip) and potential for meeting a wide range of applications requirements in different scenarios whereas molecular nano-communications are mainly restricted to biological environments 27.

Although these two nano-communication paradigms have been already depicted by the scientific community, there are still different issues that require strong efforts to make Wireless Nano-Sensor Networks (WNSN) a reality. With this objective in mind, this thesis emerges to address and offer solutions to these 
aspects always focused on EM nano-communications, which can be included in different fields of engineering (hardware, radio channel, network protocols, mathematical optimization, among others). In the following paragraphs, we deepen in these aspects, framing them in three main sections:

\section{$1.1 \quad$ Nano-device hardware}

The development of new protocol architectures for nano-communications is closely related to the capabilities of nano-devices. This issue is paramount to estimate the feasibility of, e.g., more or less complex protocols. Error correction or flow control techniques, applied in traditional WSN, may be unfeasible for this type of devices due to their limited capabilities. Even the fact of just storing information and processing it could pose a challenge. Following this premise, in [1] a first conceptual design of a nano-device with radio communication capabilities was made. However, technologies related to the manufacture of nano-components are advancing at a frenetic speed, since they use materials that until recently were in their early stages of development. Therefore, the first block of the thesis focuses on the continuous review of the most advanced technologies in nanoelectronics, as well as new materials for the manufacture of nano-antennas and nano-energy generators.

With the development of new manufacturing techniques together with materials that allow greater miniaturization without the appearance of undesired effects, it is expected that these nano-devices with communication capabilities can reach sizes of a few micrometers or even less, as analyzed in 28. These small dimensions would enable the implantation of nano-devices in scenarios where larger artificial devices would not meet the application requirements, for example, to transport a particular molecule to a given cell in the human body or to travel through the human circulatory system monitoring medical parameters, which would provide a new tool for the detection and treatment of different diseases. This case study requires further technological development, but with a device that integrates future technologies, with an appropriate network structure, and a specific communication protocol, would offer an ideal platform to achieve this application in the not too distant future.

\subsection{Characterization of the communication channel in biological scenarios}

The interior of the human body is one of the potential environments where a network of nano-devices can be deployed, as the medical applications that can be performed by a nano-network are extraordinary. Therefore, the characterization and modeling of the communication channel in biological structures (e.g. a hand or a leg) in the $\mathrm{THz}$ band becomes crucial for the development of communication protocols for WNSN. In particular, we seek to study in-depth three effects that appear in the channel: (i) molecular absorption, (ii) molecular noise, and (iii) effect of interfaces between tissues. 
Molecular absorption occurs when the medium is composed of a high percentage of water, as in biological tissues. This effect, produced by the excitation of the surrounding molecules, generates losses in the communication channel, estimated at around $50 \mathrm{~dB} / \mathrm{mm}$ at $1 \mathrm{THz} 29]$. This implies that the transmission power of a nano-device has to be very high to cover distances of a few millimeters, which is a great challenge considering the limited energy available. In the same way, this effect causes noise in reception, called molecular noise, which makes it difficult to decode the signal. Both losses and noise can be calculated mathematically in an approximate way, but they mostly depend on the composition and structure of the environment, aspects that will be taken into account in the analytical study of this thesis. In addition, the simulation of these parameters using specific software is key to the correct characterization of the environment. Following the same methodology, it will be possible to recreate the effect of the interfaces between biological tissues, that is, the passage of the EM wave from one type of tissue to another, which, to our knowledge, has not been modeled in the $\mathrm{THz}$ band.

\subsection{EM nano-communication techniques and nano-network architectures}

Due to the extremely limited computing capabilities and energy consumption of a nano-device, it is necessary to develop new communication protocols. At this point, a classic network architecture, such as those used in WSN deployments, cannot be directly applied, since a nano-device does not have the computing capacity required to support all the tasks derived from a standard protocol stack. Therefore, the third block of this Thesis focuses on the design of novel architectures for EM nano-communications with the minimum functionalities that guarantee a reliable communication between nano-devices and between the WNSN and the outside, that is, standard devices such as a smartphone or a PC. Even though the classical distinction between protocol layers is faded because of the limited features of nano-devices, we divide the study of WNSN into two essential layers: (i) physical layer together with access to the medium and (ii) routing layer.

The first layer comprises the traditional physical levels and various functionalities of the link level of the OSI model. It includes low-level communication techniques, such as modulation, medium access procedures, flow and error control, or synchronization between nano-devices. This level is especially relevant in nano-communications, since the expected operation frequencies are in the $\mathrm{THz}$ band, and the antenna would be so small that it would only resonate in this band. It should be noted that communications at this frequency are still in an embryonic stage, and no commercial device currently uses this band because of its high attenuation. However, the $\mathrm{THz}$ band offers great possibilities such as a large available bandwidth that can be used to design new modulation techniques that achieve greater energy savings than those obtained with conventional techniques. 
The second layer has the functionalities to route the information, since it is in charge of the communication between devices in the nano-network as well as the data output to the outside. In this layer, multi-hop techniques can be implemented to make the data generated by a nano-sensor reach the destination through successive hops between nano-devices in the network. Here, a hierarchical network would be proposed in which a large number of nano-devices can send data (e.g., if these nano-devices were located inside the human body, they would send medical data such as the body temperature), which are grouped into clusters, each of them controlled by a nano-router (a device that would have a larger size, computing capacity, and energy autonomy) and these in turn connected wirelessly to a nano-device that acts as a gateway (gateway in charge of dispatching the gathered data to the medical personnel) 10. This network structure, which is very common in traditional WSN, is a challenge for WNSN due to the low energy available in each nano-device and the short communication range derived from using $\mathrm{THz}$ frequencies, which makes the synchronization an essential task to ensure that the nano-devices are active at the right time.

Thus, within this block, the management of the energy available in each nano-device must also be studied, due to its vital importance for the proper functioning of a WNSN. These nano-devices do not have replaceable batteries and recharging each one of them individually would not be feasible. Therefore, they must have an energy regeneration system based on piezoelectric materials that transform mechanical movement and vibrations into electrical energy. In addition, another difference of these nano-devices compared to the sensors that comprise standard WSN is that they use capacitors instead of batteries to store energy [1, 30]. Therefore, it is required to thoroughly study concepts related to energy consumption and harvesting, such as: (i) optimal capacity of the capacitor, (ii) maximum stored energy, (iii) active and idle periods per charge cycle, (iv) optimal charge percentage. The optimization of these parameters has a clear impact on the design of nano-communication protocols that ensures acceptable network metrics, such as proper throughput, low latency, or low error rate. 



\section{Chapter 2}

\section{Objectives}

\subsection{General objectives}

Even though the global objectives of this thesis have been outlined in the previous section, here both the general goals and the specific objectives of each one of the papers published are specified. It is worth noting that the general objectives of this thesis are perfectly aligned with the goals set to be achieved in the EM nano-communications field. This ensures that all the objectives accomplished in this thesis are in line with the efforts made by the international scientific community. Overall, six global objectives were pursued in this thesis:

1. To positively contribute to the development of nanodevices endowed with communication capabilities (focused on the use of the $\mathrm{THz}$ band) and better define its specific features to be able to handle an ad-hoc nanocommunication protocol. This objective will be pursued in paper 1 .

2. To positively contribute to the development of realistic nano-network architectures and communication techniques to overcome the high path loss in biological environments with the aim of promoting the applicability of nano-communications in medicine. This objective will be pursued in paper 3 .

3. To positively contribute to standardization tasks in the field of EM nanocommunications. This objective will be sought in papers [2] and [5].

4. To positively contribute to the development of novel and more realistic energy consumption and harvesting models for EM nanodevices, with special emphasis on energy models for medical applications. This objective will be pursued in papers [4] and 3 .

5. To positively contribute to the development of transmission policies specially adapted to EM nanodevices that optimizes the management of their extremely limited resources. This objective will be pursued in paper [6]. 
6. To positively contribute to the design and implementation of novel analytical models and scenarios to accurately predict and evaluate the performance of EM nano-networks deployed inside the human body. This objective will be pursued in papers $[6$ and $[7]$.

Regarding the way these six general objectives are pursued in this work, it is worth noting that, as previously indicated in the Introduction, this thesis aims to contribute to the nano-communication arena from a realistic and viable approach. Keeping in mind that this research field is still in an earlystage, since experimental nanodevices with communication capabilities are not available yet, we have always tried to face every problem from a more realistic point of view than most of the prior works in the related literature. For that purpose, we have not only focused on isolated theoretical models based on other previous also theoretical values (an issue that caused the release of the IEEE 1906.1 standard), but we have deeply investigated each value employed in our research (i.e. energy storing and harvesting capacities, communication range, or memory available, among others) and used it always considering the worst-case to be sure that the application and model proposed are as realistic as possible. In addition, we do not study each one of the typical OSI stack layers (physical layer, data link layer, network layer...) in isolation since the scarce resources of EM nanodevices in terms of energy, memory, processing, and communication range make, from our point of view, this classical distinction quite blurred. Instead, we have aimed at designing novel crosslayer nano-communication techniques and nano-network architectures that embrace the typical three lower OSI layers at once to make the nano-network feasible and useful for the application and scenario under study.

Thus, given the novelty of the EM nano-communication field, we first devoted our efforts to acquire an in-depth knowledge about how THz-based nanocommunication works and what are the specifications of these groundbreaking nanodevices. From this approach, the first article was conceived, in which we develop a conceptual design of an EM nanodevice based on theoretical but realistic technological solutions for each component. Based on the specifications extracted from this article, we could tailor different communication protocols and techniques to the peculiarities of nanodevices that were published in the following papers.

Relying on this knowledge acquired in our first work, we realized that, whilst there were many applications proposed by different authors (softwaredefined metamaterials 31], on-chip communications [32], plants monitoring and plagues defeating systems [33, ultrahigh sensitivity touch surfaces, or chemical defense [10]), the most attractive and feasible for us, based on the features of an EM nanodevice, were those related to medicine. This way, we aim our work at improving the knowledge of how EM waves at $\mathrm{THz}$ frequencies behave in biological environments and how a nano-network should be designed and deployed to provide a useful medical application that boosts the interest of both the scientific community and medical private companies in EM nano-communications. Consequently, this goal led us to thoroughly study each 
factor impacting on the design of a realistic medical application employing EM nano-devices and increase the performance of in-body nano-networks, which motivated objectives 2 to 6 and all the following publications.

\subsection{Specific objectives}

Once the general objectives are introduced, we continue with a detailed description of how they are achieved through the seven presented articles. Although each of these papers seeks the achievement of their respective specific objectives, all of them are always related to the 6 general objectives. To understand how each article contributes to this thesis, in the paragraphs below, the specific objectives attained by each article are detailed as well as how they contribute to each of the 6 general objectives.

First Publication: Conceptual design of a nano-networking device

- Specific Objective 1A: Perform a thorough state-of-the-art review to select the appropriate technological solutions for all the components comprising a nano-device.

- Specific Objective 1B: Design a conceptual but realistic layout of a functional red-blood-cell-sized nanodevice considering the severe area limitations and the characteristics of each selected technology.

- Specific Objective 1C: Determine the main features of a red-blood-cellsized nanodevice with communication capabilities in the $\mathrm{THz}$ band. We ensured that the nanodevice had the required characteristics to operate in a WNSN.

Second Publication: The IEEE 1906.1 Standard: Nanocommunications as a new source of data

- Specific Objective 2A: Perform a detailed study of the last released version of the IEEE 1906.1 standard and envisage how it can be applied to the design of EM nano-communication protocols.

- Specific Objective 2B: Detect remarkable lacks in the IEEE 1906.1 standard that could potentially restrict its usability in EM nano-communications.

- Specific Objective 2C: Propose some guidelines and metrics to be included in future versions of the standard.

Third Publication: A nanoscale communication network scheme and energy model for a human hand scenario

- Specific Objective 3A: Design a hierarchical EM nano-communication network able to perform real-time monitoring of the human body. 
- Specific Objective 3B: Study the biological communication channel at the $\mathrm{THz}$ band simulating the dorsum of a human hand.

- Specific Objective 3C: Perform an ad-hoc communication scheme adapted to the energy limitations of nano-devices.

- Specific Objective 3D: Evaluate the performance of the entire nanocommunication network to ensure its technological feasibility.

Fourth Publication: On the Nature of Energy-Feasible Wireless Nanosensor Networks

- Specific Objective 4A: Identify and analyze the most relevant factors impacting on the energy consumption and harvesting in EM nano-communication networks.

- Specific Objective 4B: Perform a set of analytical expressions to characterize the performance of the powering system in a nano-device.

- Specific Objective 4C: Provide a detailed analysis of the energetic feasibility of an EM nano-communication network in high-lossy environments.

Fifth Publication: The IEEE 1906.1 standard: Some guidelines for strengthening future normalization in electromagnetic nanocommunications

- Specific Objective 5A: Provide a comprehensive analysis of both the definition of "nanoscale communication network" and the framework included in the released version of the IEEE 1906.1 standard from the EM nano-communications perspective.

- Specific Objective 5B: Reveal all the detected gaps that make the standard excessively generic to be embraced by the scientific community.

- Specific Objective 5C: Propose a detailed set of recommendations for each gap that should be addressed in future versions of the IEEE 1906.1 standard.

Sixth Publication: Optimal Transmission Policy Derivation for IoNT Flow-Guided Nano-Sensor Networks

- Specific Objective 6A: Derive optimal transmission policies for flow-guided nano-networks that optimize the resources of EM nano-devices.

- Specific Objective 6B: Provide a generic mathematical framework to derive smart transmission policies for flow-guided nano-networks for different applications and scenarios.

- Specific Objective 6C: Design a realistic case of study based on the monitoring of the human circulatory system to evaluate the performance increase of using proposed transmission policies. 
- Specific Objective 6D: Simulate the proposed case of study employing the derived policies and comparing them to alternative transmission policies under different scenarios.

Seventh Publication: An Analytical Approach to Flow-Guided Nanocommunication Networks

- Specific Objective 7A: Develop an analytical model to predict the performance of a flow-guided nano-network without the need of time-consuming simulations.

- Specific Objective 7B: Provide a set of theoretical boundaries to the achievable throughput of a flow-guided nano-network as a function of different parameters.

- Specific Objective 7C: Calculate the optimal number of nano-nodes to be employed in a flow-guided nano-network to maximize its throughput. 



\section{Part II}

\section{Original Articles}



Chapter 1

Conceptual Design of a Nano-Networking Device 


\title{
Article
}

\section{Conceptual Design of a Nano-Networking Device}

\author{
Sebastian Canovas-Carrasco, Antonio-Javier Garcia-Sanchez *, Felipe Garcia-Sanchez \\ and Joan Garcia-Haro
}

Department of Information and Communication Technologies, Universidad Politécnica de Cartagena (UPCT) Campus Muralla del Mar, E-30202 Cartagena, Spain; sebas.canovas@upct.es (S.C.-C.);

felipe.garcia@upct.es (F.G.-S.); joang.haro@upct.es (J.G.-H)

* Correspondence: antoniojavier.garcia@upct.es; Tel.: +34-968-326-538

Academic Editor: Leonhard M. Reindl

Received: 7 October 2016; Accepted: 6 December 2016; Published: 11 December 2016

\begin{abstract}
Nanotechnology is an emerging scientific area whose advances, among many others, have a positive direct impact on the miniaturization of electronics. This unique technology enables the possibility to design and build electronic components as well as complete devices (called nanomachines or nanodevices) at the nano scale. A nanodevice is expected to be an essential element able to operate in a nanonetwork, where a huge number of them would coordinate to acquire data, process the information gathered, and wirelessly transmit those data to end-points providing innovative services in many key scenarios, such as the human body or the environment. This paper is aimed at studying the feasibility of this type of device by carefully examining their main component parts, namely the nanoprocessor, nanomemory, nanoantenna, and nanogenerator. To this end, a thorough state-of-the-art review is conveyed to discuss, substantiate, and select the most suitable current technology (commercial or pre-commercial) for each component. Then, we further contribute by developing a complete conceptual nanodevice layout taking into consideration its ultra-small size (similar to a blood cell) and its very restricted capabilities (e.g., processing, memory storage, telecommunication, and energy management). The required resources as well as the power consumption are realistically estimated.
\end{abstract}

Keywords: nanodevice; wireless nanosensor network; terahertz band; nanotechnology; ultra-low power device

\section{Introduction}

In recent decades, the technological advances in novel materials have enabled a new generation of increasingly smaller electronics, which have become fundamental tools for the future development of components such as processors, batteries, and sensors/actuators. Ultimately, the downsizing of electronics has led to a new paradigm, the so-called nanodevice. A nanodevice is conceived to be sized on a scale of a few nanometers. This novel nanomachine is drawing broad interest from the scientific community, since nanodevices can operate at the nanoscale as nanosensors and/or nanoactuators, thus opening the analysis of different, unforeseen essential parameters and magnitudes, such as hormone levels, disease detection, control of bio-implants in human/animal bodies or air pollution measurements in the atmosphere, among others. This fact may allow the observation of currently unstudied scenarios, enabling a plethora of potential applications in fields as different as biomedicine, environmental science or industry. Nevertheless, nanodevices must go way beyond, to provide additional capacities for attaining a complete technological solution, which can dispatch the acquired and further processed information to remote end destinations. Therefore, as stated in [1], the expected nanodevice capabilities must encompass many aspects, such as sensing/acting, processing, energy management, and telecommunications. 


\section{CHAPTER 1. CONCEPTUAL DESIGN OF A NANO-NETWORKING DEVICE}

To reckon these expectations, new challenges have arisen in order to propose a nanodevice that can be feasibly deployed in real scenarios. These concerns are mainly posed by the nanoscale nature, which steeply restricts the component size, amount of available resources, capabilities, and performance. For instance, the limited size of nanobatteries has a direct impact on the amount of energy that they can store. This fact, together with the impossibility of accomplishing, in many scenarios, manual recharges or replacements, negatively affects the operational lifetime of the whole nanodevice. In addition, the energy consumption of a nanodevice should be ultra-low for it to be to be powered efficiently by a nanobattery. On the other hand, the miniaturization of classical antennas to meet the size requirements imposes the use of extremely high operating frequencies (expected to be in the terahertz band). However, this band suffers from high propagation losses, which, in turn, limit the communication range between nanodevices to only a few millimeters. Finally, the very restricted computing capacity along with the scarcity of available memory will also limit the volume of information that a nanodevice can handle.

To tackle these limitations, nanodevices cannot operate in isolation; rather, they have to be grouped into what is called nanonetworks/wireless nanosensor networks (WNSNs). A WNSN allows single nanodevices to collaborate and share information among them. As a result, these nanonetworks could cover larger distances and carry out more complex tasks in nanoscale environments. Communications in nanonetworks will be possible thanks to a robust and scalable communication protocol, which must provide a set of straightforward functionalities. They have to be addressed to ensure a reliable communication in a planned environment consisting of a potentially huge number of nanodevices, without being jeopardized by their strong individual restrictions in computing, memory, and power consumption. Therefore, there is a need to conceive a nanodevice that offers, among other functionalities, the capability to satisfy reliable communications between a nanodevice and its neighbors in the coverage area. In this paper, we place special emphasis on the communications aspect, but without going into detail on the design of any particular communication protocol stack.

The objective of this paper is thus to know and better understand the electronics associated with the main nanocomponents required for a nanodevice, its layout as well as its key specifications. To do so, we start from the seminal work by Akyildiz and Jornet [2], who conceived a nanodevice architecture including radiocommunication capabilities. However, the lack of quantitative specifications, such as processor resources, clock frequencies, the amount of available memory or total energy consumed, makes it difficult to demonstrate the feasibility of a nanonetwork. In this paper, we go one step further and we contribute with a more detailed and quantified conceptual design of the main components that integrate a nanodevice using current technologies (by current we mean commercial or pre-commercial technologies). This nanodevice will have the sufficient capability to operate in a WNSN.

To this end, as a first contribution, we provide an insightful state-of-the-art review to select the appropriate technological solutions for the main components comprising a nanodevice. We have divided the nanodevice into four main different components-nanoprocessor, nanomemory, nanoantenna, and nanogenerator - following the lines devised by Akyildiz and Jornet [1]. Regarding this issue, and in accordance with conceptually designing a nanodevice employing up-to-date electronic technologies, we note that the communications surveyed in this work involve the transmission of information via electromagnetic (EM) waves [3] and not with molecules-based communications [4]. Once the technology of each component is described in depth, our second ambitious goal is to conceptualize the nanodevice design, quantifying, on the one hand, its size/dimensions and, on the other hand, the core features for each of the four main components integrating the nanodevice. Special attention has been paid to the communication tasks that a generic nanodevice has to fulfill in the WNSN. For the size of the nanodevice, we have taken as a reference a red blood cell, whose typical measurements are approximately $8 \times 8 \times 4 \mu \mathrm{m}$, which fits the conceived size for a nanodevice [1]. Figure 1 shows a likely environment under consideration, while Figure 2 illustrates our conceptual design for a nanodevice. To the best of our knowledge, this 
is the first work that deals with quantifying the resources required by a generic nanodevice working in a WNSN.

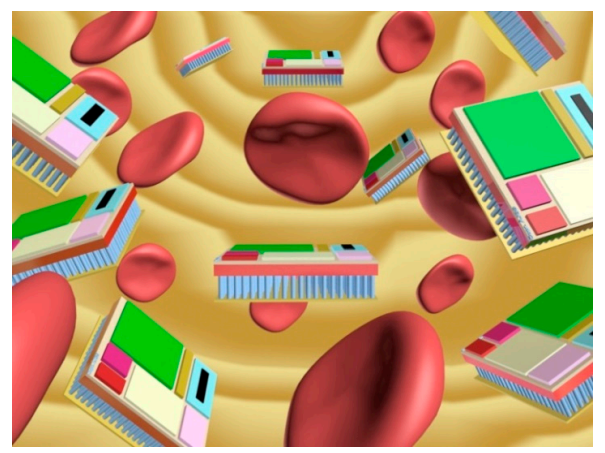

Figure 1. Nanodevices flowing through the bloodstream.

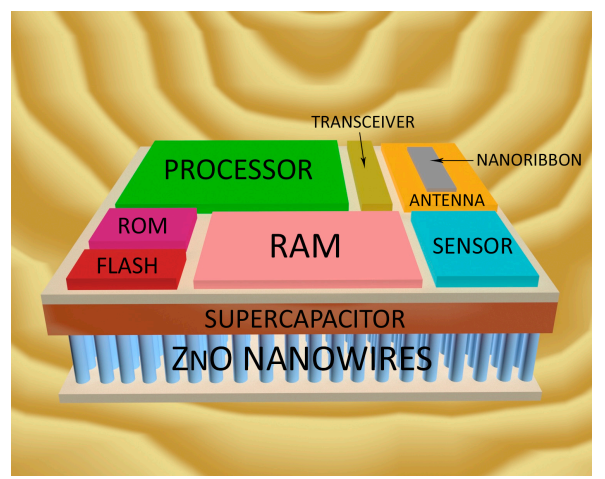

Figure 2. Nanodevice-a general view of our proposal.

The paper is organized as follows: Section 2 reviews the technological solutions for each component of a nanodevice, analyzing its advantages and drawbacks and studying the feasibility of integrating them in the complete nanodevice system. Under these considerations, we establish an appropriate trade-off technological solution for every electronic component in terms of performance, scalability, power consumption, and current state of the technology. In Section 3, we propose our nanodevice layout sizing all the components. We also provide an energy consumption analysis to ensure the feasibility of the nanodevice operation, as well as point out its principal energy limitations. Finally, Section 4 concludes the paper.

\section{Technical Background}

To undertake the design of a nanodevice, different current technologies must be carefully analyzed in order to select a reasonable solution for each component. An end-technological decision per component is a key issue because it directly affects the capabilities and feasibility of the complete nanodevice. For instance, having enough memory and processing resources could enable interesting functionalities and more consistent communication tasks. Therefore, in this section, we review in depth the state-of-the-art for the four principal components composing a nanodevice, namely: (i) 


\section{CHAPTER 1. CONCEPTUAL DESIGN OF A NANO-NETWORKING DEVICE}

nanoprocessor; (ii) nanomemory; (iii) nanoantenna; and (iv) nanogenerator. Since, a priori, multiple technologies can be potential candidates to be part of the nanodevice, we will analyze them, remarking on their pros and cons as well as quantifying their resources per area. Finally, based on their main features, we select the more suitable current technology for each component, which is crucial to ensure a suitable nanodevice performance.

\subsection{Nanoprocessor}

The nanoprocessor is the nanodevice component that will drive all the remaining nanodevice hardware (with the exception of the nanogenerator and nanobatteries). Having in mind this premise and the limited available nanodevice area, its design must satisfy both its own functionality and the operation of the components that directly depend on it. To fulfill these requirements, the nanoprocessor must contain an appropriate number of tiny transistors (the basic element of a processor). Hence, we focus our attention on the typical physical parameters of a transistor, such as the feature size or its area, to carry out a reliable estimation of the number of them needed, which in turn, is in accordance with the required nanodevice capabilities. Moreover, we also consider the energy consumption that is entailed, since, as will be emphasized, it is a critical aspect in the nanodevice design. Then, we review the related transistor manufacturing technologies, comparing their scalability, power consumption and fabrication process. Once they are all examined, we summarize their most relevant features in Table 1, noting their pros and cons.

Table 1. Comparison of nanoprocessor technologies.

\begin{tabular}{|c|c|c|c|c|}
\hline $\begin{array}{l}\text { Transistor } \\
\text { Technology }\end{array}$ & $\begin{array}{c}\text { Minimum } \\
\text { Transistor Size }\end{array}$ & Advantages & Disadvantages & Feasibility \\
\hline \multirow{2}{*}{ Silicon } & \multirow{2}{*}{$14 \mathrm{~nm}$} & Mature technology & \multirow{2}{*}{ Scalability concerns } & \multirow{2}{*}{ Yes } \\
\hline & & Low-cost manufacturing & & \\
\hline \multirow{3}{*}{ SiGe } & \multirow{3}{*}{$7 \mathrm{~nm}$} & Good scalability & \multirow{3}{*}{$\begin{array}{l}\text { Experimental } \\
\text { technology }\end{array}$} & \multirow{3}{*}{ Yes } \\
\hline & & Low-cost manufacturing & & \\
\hline & & Ultra-low power consumption & & \\
\hline \multirow{3}{*}{ CNT } & \multirow{3}{*}{ Sub $20 \mathrm{~nm}$} & Great scalability & $\begin{array}{l}\text { Experimental } \\
\text { technology }\end{array}$ & \multirow{3}{*}{ Yes } \\
\hline & & Ultra-low power consumption & Difficult manufacturing & \\
\hline & & High speed & process & \\
\hline Atomic & One atom thick & Ultra-small size & $\begin{array}{l}\text { Operation under strict } \\
\text { laboratory conditions }\end{array}$ & Not yet \\
\hline
\end{tabular}

2.1.1. Silicon-Based Technologies

Nowadays, silicon-based transistor solutions are mature technologies addressed to manufacture and commercialize all kind of electronic devices. Since the first commercial processor - with a feature size of $10 \mu \mathrm{m}$-was launched on the market [5], many companies have developed novel tiny chips, highlighted by their high processing capabilities and low power consumption. Thus, over the last three decades, as the transistor size has become progressively smaller, the number of transistors in a chip practically has doubled every two years (Moore's law has been accomplished "quasi" faithfully). Smaller transistors influence the chip design from a two-fold perspective. On the one hand, a large number of transistors can be packed in the same chip area, resulting in more functional and powerful microprocessors. On the other hand, there is the challenge of addressing the development of new tiny chips with the same or better capabilities than a microprocessor manufactured some years ago [6].

However, due to the increasingly larger channel length reduction of the metal oxide semiconductor field-effect transistor (MOSFET), some undesired effects arise, known as short-channel effects [7], revealing that the silicon technology is reaching its limit. One of the most important concerns is the subthreshold leakage current effect [8], which makes the design of low power consumption transistors difficult. This effect appears when the channel length shrinks. In this 


\section{CHAPTER 1. CONCEPTUAL DESIGN OF A NANO-NETWORKING}

case, the voltage applied to the gate in order to switch the transistor to the "on" state is lower affecting the threshold voltage (upper bound value that generates a current between the source and the drain), which also decreases. Under these conditions, the voltage range between the "on" and "off" states is tight, impairing the transistor to switch to a complete "off" state. This generates a source-drain current even though the voltage is below the threshold. This subthreshold current was not a problem in previous commercial transistors since they considered higher channel lengths and, therefore, a large threshold voltage. However, for tiny solutions, this undesirable current poses an important downside because, when the transistor operates below $1 \mathrm{~V}$, a substantial fraction of the total energy consumed is lost, wasting a significant amount of energy [9].

The leading semiconductor companies have developed novel transistor architectures to mitigate the undesirable behavior of the short-channel effect, increasing the channel size and benefitting from the third dimension. These non-planar architectures, called FinFET [10] or 3D Tri-gate transistor [11], depending on the company, also incorporate more than one gate for each transistor. This feature, along with the possibility of implementing a third dimension, results in reducing the transistor size $-14 \mathrm{~nm}[12]-$ and, thus, higher transistor density chips. FinFETs were reported for the first time in 1999 [13], but they have not been implemented in commercial processors until recently.

Unfortunately, 3D transistors-focused on powerful processors-are not conceived for applications where the energy savings is a primordial requirement. In this sense, transistors based on planar architectures are commonly used (note that they restrict the number of traditional silicon MOSFET available in a nanoprocessor). As an example, in current state-of-the-art sensor networks, the ARM Cortex-M0+ [6], fabricated with 40-nm lithography, is one the most energy-efficient microprocessors on the market [14]. The dynamic power in its specifications [15], that is, the power consumed when the processor is in active mode, is $3.8 \mathrm{nW} / \mathrm{kHz}$ operating at $1.1 \mathrm{~V}$. Hanson et al. [16] designed and implemented an experimental processor, called Phoenix, that consumes $2.8 \mathrm{nW} / \mathrm{kHz}$ at $0.5 \mathrm{~V}$, which is considered a reference in minimum power consumption.

Therefore, a trade-off between the transistor size and its power consumption is required to design a nanoprocessor granting less area per transistor (and thus, packing the maximum number of transistors in an enclosed chip area) without compromising its operational lifetime. To accomplish this trade-off solution, new semiconductor materials instead of silicon must be considered. These materials are appraised in the following subsections.

\subsubsection{Silicon Germanium (SiGe)-Based Technologies}

To improve channel length scaling and performance in MOSFET transistors, the electron mobility $(\mu)$ of a material is a crucial parameter to take into consideration. In this regard, Equation (1) expresses the current $(I)$ that flows along a MOSFET as a function of the electron mobility [9]:

$$
I \approx \mu \frac{W}{L_{c h}} C_{o x}\left(V_{g s}-V_{t}\right) V_{d s}
$$

where $V_{g^{s}}$ is the gate-source voltage, $V_{t}$ is the threshold voltage, $V_{d s}$ is the drain-source voltage, and $L_{c h}$ and $W$ are the length and width of the channel, respectively. Finally, $C_{o x}$ is the oxide-gate capacitance.

One can observe that if the expression $\mu \frac{W}{L_{c h}} C_{o x}$ increases, the $V_{g s}, V_{t}$ and $V_{d s}$ parameters have to reduce their values to maintain the same amount of current flowing. To increase the value of $\mu \frac{W}{L_{c h}} C_{o x}$, the only channel parameter that can vary is the electron mobility $(\mu)$, since the remaining ones are design constants related to the channel dimensions. Therefore, a larger velocity of the electrons improves the transistor performance in terms of power consumption [17].

One promising material that boosts electron mobility in the channel is silicon-germanium alloy $[9,18]$. For this type of material, the parameter $\mu$ has a high value that, in accordance with the paragraph above, results in transistors operating at very low voltages and, therefore, the energy waste is reduced in comparison with silicon-based transistors. 


\section{CHAPTER 1. CONCEPTUAL DESIGN OF A NANO-NETWORKING DEVICE}

Another benefit of SiGe technology is related to the cost saving in the manufacturing process. For SiGe transistors, the costs associated with their fabrication are similar to traditional silicon technology [19]. Therefore, from both an economic perspective and a technological solution, the SiGe appears as an appropriate replacement for the silicon-based technology.

Under these premises, IBM launched the first $7 \mathrm{~nm}$ transistor based on the SiGe alloy [20], thus validating its feasibility and potential. With this new technology it is viable to include in a chip the size of a fingernail more than 20 billion transistors. This figure is for an area per transistor of 5000 $\mathrm{nm}^{2}$, supposing a clear advance to develop tiny nanoprocessors.

\subsubsection{Carbon Nanotubes}

Carbon nanotubes (CNTs) consist of a single sheet of rolled carbon atoms forming a hollow cylinder structure of approximately $1 \mathrm{~nm}$ in diameter. Among their design attributes, these simple nanocylinders have noteworthy electrical and physical properties [21-23], which can be very useful for building field-effect transistors (FETs). In these CNT-based FETs, the channel is created by nanotubes attaching the source and drain (metallic bilayers composed of palladium and platinum) [24]. CNTs present two advantages; first, high charge-carrier mobility, resulting in a faster "ON-OFF" switching speed in comparison with SiGe and silicon-based transistors [25,26], and second, a significantly lower subthreshold leakage current than the two previous technologies [9].

CNT potential and limitations are thoroughly discussed in the specialized literature [9,27-29]. The latter are due to the difficulties in accurately placing nanotubes on the substrate. Earlier positioning techniques addressed the angular misalignment of nanotubes, which noticeably affected the transistor channel lengths built in the chip. The result was an unacceptable distortion of the transistor performance since CNT-based transistors would have different channel lengths, which could have led to the failure of the electronic circuit [30]. Nowadays, this handicap has been overcome since new positioning techniques [30] improve the placement of a large number of CNT-based transistors on a single chip.

To prove the feasibility of this technology, a computer-based prototype was fabricated employing CNT field-effect transistors (CNFET) [24]. Each CNFET is composed of a variable number of carbon nanotubes, ranging from 10 to 200, which are appropriately aligned. Using this configuration, CNFET obtain the same performance independently of the number of CNT. The authors pointed out that this effect is due to intrinsic problems of the academic fabrication facilities employed. Therefore, it is reasonable to think that more efficient results can be reached as fabrication methods improve. Along these lines, the work in [27] assumes that the ideal CNFET must only contain one CNT between source and drain, therefore reducing the CNFET size.

Hence, CNFET are a promising technological alternative, which would outperform the traditional silicon MOSFET by reducing the transistor size and improving energy efficiency. In particular, CNFET power consumption is estimated one order of magnitude lower than that of silicon-based transistors [23].

\subsubsection{Atomic Technology}

The ability to build nanocomponents on the atomic scale is envisaged as the future of nanotechnology. Unfortunately, current techniques and tools to fabricate chips with atomic precision are in an embryonic state. However, in recent years, the scientific community has reported some progress, in particular, regarding the fabrication of the first single-atom transistor [31]. The channel of this transistor is achieved by just one phosphorous dopant atom placed over a silicon crystal. In addition to this atom, four phosphorous-doped electrodes operate as source, gate and drain. The authors employed a combination of scanning tunneling microscopy and hydrogen-resist lithography to build the single-atom transistor with atomic accuracy. In experimental tests, this transistor operates at cryogenic temperatures so the development of a functional electronic device based on this technology is, at this moment, unfeasible.

\subsubsection{Technology Selection}




\section{CHAPTER 1. CONCEPTUAL DESIGN OF A NANO-NETWORKING}

Once analyzed the main technologies related with the design and implementation of transistors, we concluded that silicon-based and molecular solutions are not appropriate due to their poor scalability and technical unfeasibility, respectively. On the other hand, transistors based on SiGe alloys and CNTs are suitable alternatives in order to design a future nanoprocessor. CNTs present an excellent scalability along with a great potential to reduce both transistor size and power consumption. However, the main problem against this technology is its manufacturing process, since it requires an exhaustive accuracy in the positioning of each CNT into a CNFET. While we note that signifying advances are being achieved [24], the CNT-based technology still needs more research to be a real option.

For continuing with our study, we advocate the SiGe technology since it is currently the most feasible solution to design the future nanoprocessor. It is true, however, that the features of SiGe to achieve smaller MOSFET are not as suitable as, for instance, those of CNTs. Likewise, the SiGe-based transistor has enough abilities to obtain a functional nanoprocessor satisfying the expected nanodevice requirements (read data from the sensor, memory write/read operations, and executing a simple ad-hoc communication protocol), as we will discuss in Section 3 . In this sense, the SiGe-based chip fabricated by IBM is the first approach that groups all the advantages of the SiGe technology. With a $7 \mathrm{~nm}$ technology, the SiGe-based chip leads as the basis of the design and development of future nanoprocessors. Furthermore, SiGe chips can be manufactured employing the same tools as the traditional silicon transistors. Therefore, the costs to fabricate a SiGe-based nanoprocessor are significantly lower in comparison with other emerging technologies. Regarding power consumption, IBM points out that SiGe chips will have at least a $50 \%$ improvement with respect to actual silicon-based solutions [20].

\subsection{Nanomemory}

The storage capacity of an electronic device is an important aspect, because the amount and complexity of the stored programming code relies directly on the available memory. This has an impact on most nanodevice functionalities as, for instance, the communication protocol stack. In this sense, many of its configuration parameters (such as device ID length, packet size, number of bits for error detection, etc.) intrinsically depend on the available memory.

As we will discuss in Section 3, the nanoprocessor should work at low frequency to reduce the power consumption. Unlike traditional memory designs where the write and read access times are a critical concern, the low-frequency operation plays a major role in our nanomemory design, with the write and read times becoming a secondary aspect. Under these circumstances and as it occurs for the remaining components of a nanodevice, the constrained area devoted and the power consumed are the most important issues to overcome. Thus, the key aspects to consider in our study are the cell area size along with the energy required to store a bit. In this section, we review the most remarkable and actual memory technologies, dividing them into two groups: non-volatile and RAM. For each group, we analyze different types of memory and their task into the nanodevice. Tables 2 and 3 specify the main features for each type together with their most notable advantages and drawbacks.

Table 2. Comparison of non-volatile memory technologies.

\begin{tabular}{|c|c|c|c|c|}
\hline Storage Technology & Cell Size & Advantages & Disadvantages & Feasibility \\
\hline \multirow{3}{*}{ NAND Flash SLC } & \multirow{3}{*}{$784 \mathrm{~nm}^{2}$} & Mature technology & \multirow{3}{*}{ Scalability concerns } & \multirow{3}{*}{ Yes } \\
\hline & & Low power consumption & & \\
\hline & & Low-cost manufacturing & & \\
\hline \multirow{2}{*}{ NAND Flash MLC } & \multirow{2}{*}{$392 \mathrm{~nm}^{2}$} & Mature technology & $\begin{array}{l}\text { High power } \\
\text { consumption }\end{array}$ & \multirow{2}{*}{ Yes } \\
\hline & & Low-cost manufacturing & $\begin{array}{c}\text { Low write and read } \\
\text { speed }\end{array}$ & \\
\hline \multirow{3}{*}{ NOR Flash } & \multirow{3}{*}{$1293 \mathrm{~nm}^{2}$} & Mature technology & Scalability concerns & \multirow{3}{*}{ Yes } \\
\hline & & High read speed & High power & \\
\hline & & $\begin{array}{l}\text { Low energy consumption } \\
\text { (read) }\end{array}$ & $\begin{array}{l}\text { consumption } \\
\text { (write) }\end{array}$ & \\
\hline
\end{tabular}


CHAPTER 1. CONCEPTUAL DESIGN OF A NANO-NETWORKING DEVICE

\begin{tabular}{|c|c|c|c|c|}
\hline \multirow{3}{*}{ Racetrack } & \multirow{3}{*}{$200 \mathrm{~nm}^{2}$} & Good scalability & \multirow{3}{*}{$\begin{array}{c}\text { Experimental } \\
\text { technology }\end{array}$} & \multirow{3}{*}{ Not clear } \\
\hline & & High $\mathrm{r} / \mathrm{w}$ speed & & \\
\hline & & Ultra-low power consumption & & \\
\hline \multirow[b]{2}{*}{ GMR } & \multirow[b]{2}{*}{$0.7 \mathrm{~nm}^{2}$} & \multirow[b]{2}{*}{ Excellent scalability } & $\begin{array}{c}\text { Experimental } \\
\text { technology }\end{array}$ & \multirow[b]{2}{*}{ Not clear } \\
\hline & & & $\begin{array}{l}\text { High energy } \\
\text { consumption } \\
\text { (write) }\end{array}$ & \\
\hline
\end{tabular}

Table 3. Comparison of RAM memory technologies.

\begin{tabular}{|c|c|c|c|c|}
\hline Storage Technology & Cell Size & Advantages & Disadvantages & Feasibility \\
\hline \multirow{2}{*}{ DRAM } & \multirow{2}{*}{$2900 \mathrm{~nm}^{2}$} & Mature technology & High energy & \multirow{2}{*}{ Yes } \\
\hline & & High density & consumption & \\
\hline \multirow{2}{*}{ SRAM } & \multirow{2}{*}{$64,000 \mathrm{~nm}^{2}$} & Mature technology & \multirow{2}{*}{ Low density } & \multirow{2}{*}{ Yes } \\
\hline & & Low energy consumption & & \\
\hline \multirow{2}{*}{ A-RAM } & \multirow{2}{*}{$1176 \mathrm{~nm}^{2}$} & High density & \multirow{2}{*}{ Novel technology } & \multirow{2}{*}{ Yes } \\
\hline & & Low energy consumption & & \\
\hline \multirow{2}{*}{ PRAM } & \multirow{2}{*}{$127.5 \mathrm{~nm}^{2}$} & Ultra-high density & Experimental & \multirow{2}{*}{ Not yet } \\
\hline & & High access speed & technology & \\
\hline
\end{tabular}

2.2.1. Non-Volatile Memories

Flash Memory

Nowadays, flash memory is a non-volatile memory that is present in most electronic devices to store data, even if the energy supply is cut off. Due to its low-cost manufacture, low power consumption and high access speed, flash memory is commonly used in many devices, which can be summarized into two main categories: (i) general data storage such as, for instance, memory cards, USB flash drives, or solid-state drives; and (ii) configuration data and user files storage in digital products. Depending on the logic of each memory cell, two types of flash memories have been developed by the industry: NAND and NOR flash.

A NAND flash memory stores bits in memory cells made of floating-gate MOSFET (FGMOS) [32]. These transistors are able to maintain the charge level even in the case where they are disconnected from the power supply. This is possible thanks to its particular design based on two gates, as shown in Figure 3. The first, the lower gate-denoted as floating gate (FG) -is electrically isolated by a dielectric (capacitance) from the upper gate-control gate (CG) - and the substrate. The goal is for the FG to act as a floating node under a given potential threshold in direct current (DC). Then, the FG can be seen as a potential well. The charges that fall into this potential well remain there (as if they were enclosed) until an external voltage is applied.

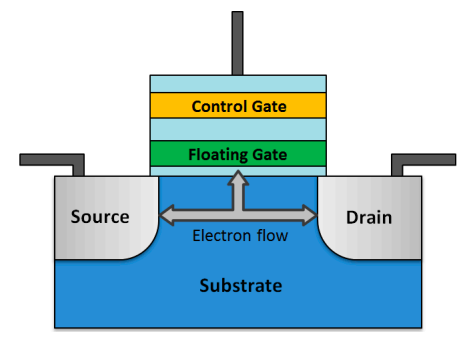

Figure 3. Cross-section of a FGMOS transistor. The transversal voltage between the channel and the control gate attracts part of the electron flow (from source to drain) to the floating gate. This 


\section{CHAPTER 1. CONCEPTUAL DESIGN OF A NANO-NETWORKING}

Among diverse mechanisms to transfer external electric charge to and from the FG, two predominate: (i) hot-electron injection (HEI); and (ii) Fowler-Nordheim (F-N) tunneling [32]. In HEI, a voltage between source and drain "heats" the electrons, providing enough kinetic energy to overcome the potential barrier. Simultaneously, a transversal voltage between channel and CG injects the charges into the FG. Likewise, an inverse voltage can be applied to the CG to remove the charges. The F-N tunneling occurs when the applied electric field between the substrate and the CG is sufficiently large to overcome the potential barrier if the FG is charged. F-N tunneling is widely employed in actual flash memories since the voltage required for the write operation is lower than in HEI-based memory cells [33-35].

Following the guidelines of the previous paragraph, a logic "1" is stored in the FGMOS when electrons are trapped in the FG, and a logic " 0 " when the FG is empty. This cell configuration is known as single-level cell (SLC), since only one bit is stored per cell. Nevertheless, novel FGMOS devices have been developed to store more than one bit per cell, thus increasing the bit density. These technologies are called multi-level cell (MLC), triple-level cell (TLC), and quadruple-level cell (QLC) [34], depending on the bits stored per cell.

The addition of more bits per cell makes it more difficult to distinguish among states, hence the read operation requires multiple stages to detect the state accurately. An SLC flash performs the read operation in only one stage, which involves lower read/write access time than any of the multi-level technologies [33]. In particular, in comparison with SLC technology, MLC requires up to four times more time to write and 2.5 times more to read [36]. Other parameters such as power consumption, reliability and endurance are also affected, obtaining worse values when more bits per cell are stored [36].

To figure out the energy consumption associated with SLC and MLC NAND flash technologies, we refer to the comparison between both as reported in [33]. The energy per bit estimated in the write operation using SLC is 305 pJ, whereas for MLC it is 1200 pJ. On the other hand, the read process demands significantly less energy (30 pJ per bit for SLC and $91 \mathrm{pJ}$ per bit for MLC) Therefore, during the write and read processes, MLC-based technology consumes up to four and three times more than the SLC solution, respectively.

To compare different storage technologies, the cell size is a key metric, which is typically given in units of minimum feature size (F). Concerning NAND flash, and employing a self-aligned STI (SA-STI) cell structure for optimizing the area with respect to older cell structures [34], the cell size can be scaled down to $4 \mathrm{~F}^{2}$. Moreover, using MLC technology, the effective cell size is reduced by half, reaching thus, a value of $2 \mathrm{~F}^{2}$.

The $\mathrm{F}$ value is intrinsically related to the size of the transistor. Thus, considering the most advanced silicon-based transistor $-14 \mathrm{~nm}$ [12]-the cell size for a NAND flash results in $784 \mathrm{~nm}^{2}$ using the SLC technology and $392 \mathrm{~nm}^{2}$ for MLC-based solutions. These values determine the required area to store one bit and are in the range of other novel solutions as the commercial-focused 3D high-density flash memory [37], which attains effective cell sizes of $233 \mathrm{~nm}^{2}$. However, a memory fabricated with this technology is not conceived for low energy consumption.

A NOR flash memory obeys the same electronic principles to store a bit than NAND flash, but the logic employed in each cell is different. The NOR flash cell structure is designed for reaching a better read speed than NAND flash, providing random access times below $100 \mathrm{~ns}$ [38]. NOR flash is, thus, best suited to store data, which requires a low read delay, such as the execution code.

However, the energy consumption associated with the write operation is higher in NOR flash than in NAND flash [39]. To the best of our knowledge and after reviewing the related scientific literature, no works specifying the energy consumption for the read operation could be found. Despite this shortcoming, since NOR Flash is intended to satisfy read-only applications, we can estimate the energy consumed by identifying it as a ROM memory. Thus, in [40], the ultra-low NOR ROM memory consumes $0.2 \mathrm{pJ}$ per operation, which can be taken as a value of reference to estimate 


\section{CHAPTER 1. CONCEPTUAL DESIGN OF A NANO-NETWORKING DEVICE}

the NOR Flash energy consumption. This quantity is two orders of magnitude fewer than that in NAND Flash, which is acceptable due to the read-only nature of the NOR Flash proposed.

Regarding the physical size, the results found in [35] showed that NOR flash memory is scaled down to $50 \mathrm{~nm}$ with a cell size of $6.6 \mathrm{~F}^{2}$. Considering the same $\mathrm{F}$ as the one used for NAND flash cell size estimation $-14 \mathrm{~nm}$ - the cell area of the NOR flash memory is $1293 \mathrm{~nm}^{2}$, which is approximately two times more than for NAND flash.

\section{Racetrack Memory}

Racetrack memory is an innovative non-volatile memory that uses magnetic domains to store bits along a ferromagnetic nanowire, built on a silicon substrate. Each bit is stored in a magnetized region, divided into magnetic domain walls. Pulses of electric current move the bits along the nanowire to read and write data. The nanowire has a diameter of $10 \mathrm{~nm}$ and an approximate length of $200 \mathrm{~nm}$, where these values depend on the number of total bits stored on it $[39,41]$.

It is worth noting that the read and write access times for this type of memory are faster than actual commercial memories, incurring less energy consumption. In addition, it is possible to obtain an extremely high bit density, since nanowires can be placed vertically (using the third dimension), packing a high amount of them in less area. However, this arrangement might not be appropriate for a nanodevice, since this third dimension is also tightly restricted. Under this premise, we calculate the cell size as a function of the horizontal plane, obtaining a value of $2 \mathrm{~F}^{2}$ for a feature size of $10 \mathrm{~nm}$ [39]. Therefore, the cell area is $200 \mathrm{~nm}^{2}$ around, clearly lower than the NAND flash cell area.

This technology, although encouraging, is still in an experimental phase. Therefore, more research and development is necessary in order to combine racetrack memory with standard MOSFET-based electronic circuits.

\section{GMR Effect-Based Memory}

The giant magnetoresistance (GMR) effect is a mechanical magnetoresistance detected in structures composed of ferromagnetic and non-magnetic material layers. A significant variation in the resistance of these layers (sized to molecular scale) occurs when the magnetic orientation of the ferromagnetic layers changes. The magnetization direction can be controlled by applying an external electromagnetic field [42]

Under these foundations, to write a bit, the ferromagnetic layer induces a magnetic moment in the molecules, which is retained in the molecular layer storing a logic " 1 ". Switching the orientation of the moment in the molecular layer by applying an external electromagnetic field or a high voltage, the bit is removed; that is, a logic " 0 " is written in the memory [43].

Using this technology, it is possible to achieve a storage density close to $10^{15} \mathrm{bits}_{\mathrm{inch}}{ }^{2}$ [43]. This density value involves about one bit per $0.7 \mathrm{~nm}^{2}$, which is the highest density value compared to the remaining memory technologies reviewed. Concerning the energy consumption issue, as of today, a high voltage is required to write a bit, but as far as we know, there is no open work accounting for it.

Although GMR effect-based memory has been recently patented, the viability of fabricating a device at a reasonable cost is not yet clear.

\subsubsection{RAM Module}

Another basic storage component for any electronic device is the Random Access Memory (RAM) module. RAMemory is characterized by its fast speed-more than 1000 times faster than a NAND Flash-regardless of the physical position of the data in the memory. A RAM unit is the ideal selection for current cache memories, where data handled by the processor are temporally stored. These data tend to change quickly while the processor is working, thus, high speed memories are required to meet an appropriate performance. Although the nanoprocessor will operate at low frequency-estimated in the $\mathrm{kHz}$ range-it is recommended that a RAM module be included for the most recurrent data, as used in most low-power processors [16,44-46].

Volatile RAM 


\section{CHAPTER 1. CONCEPTUAL DESIGN OF A NANO-NETWORKING}

Nowadays, the two main types of commercial volatile RAM are static RAM (SRAM) and dynamic RAM (DRAM). The major difference between them is the way they store data. DRAM stores each bit in a capacitor whose charge decreases as the time passes, thus, the charge must be refreshed periodically, consuming additional energy. However, in SRAM, a refresh signal is not required; data remain whenever the memory is powered. Under these premises, SRAM usually consumes slightly less power than DRAM. However, SRAM offers less density of bits in comparison with DRAM, whose cell size is about $2900 \mathrm{~nm}^{2}$ [47]. This is because, for a standard cell architecture, traditional SRAM contains six MOSFET-6T SRAM [16,48]-whereas DRAM employs a single transistor. The cell size reported for a 14nm FinFET SRAM cell is $0.064 \mu \mathrm{m}^{2}$ [49], which involves a feature size of $327 \mathrm{~F}^{2}$. Nevertheless, this traditional conception is changing since new SRAM and DRAM architectures [50,51] integrate features of both RAM types. In [50], the authors designed a capacitor-less DRAM cell, called A-RAM. Releasing the use of capacitors-which is the main restricting factor for further DRAM miniaturization-an A-RAM achieves smaller memory cells than their predecessors. Therefore, considering the DRAM cell size reported in [39]-6 $\mathrm{F}^{2}-$ and based on the A-RAM scaling capability, we adopt the value of $1176 \mathrm{~nm}^{2}$ as the A-RAM cell area. In addition, longer data retention times are also achieved-more than $100 \mathrm{~ms}-$ which means less refreshing frequency and, consequently, less energy consumed.

\section{PRAM Memory}

Phase-change RAM (PRAM) is a non-volatile memory that stands out for its great scalability and high speed operation. PRAM is led to compete with both volatile and non-volatile memories, since random access times are similar to DRAM-below $30 \mathrm{~ns}$. In [52], the fabrication of a PRAM module is illustrated (employing Sb-rich Ge-Sb-Te phase change material) with a $17 \mathrm{~nm}$ design rule, proving the scalability potential of the technology. The cell size achieved is $127.5 \mathrm{~nm}^{2}$. Nevertheless, this promising technology is still in the experimental stage, since there are no issued products based on this memory.

\subsubsection{Technology Selection}

In order to obtain the highest bit density preserving good reliability and low energy consumption, the best option for the non-volatile storage of our nanodevice design is the SLC NAND Flash memory. Although the bit density in MLC-based solutions is two times higher than in SLC, their average energy consumption is four times lower, which entails a significant advantage. Other promising technologies reviewed (Racetrack and GMR effect-based memories) reach higher bit densities, but their feasibility has not yet been tested in functional prototypes.

To store permanent data, such as the programming code to boot the nanodevice, a ROM module is required. NOR flash memory assures faster read access times than NAND flash, obtaining an acceptable bit density and a restrained energy consumption when reading. Under these premises, NOR flash appears as the best-suited memory for this purpose.

Finally, in reference to volatile memory, a RAM module should be integrated in the nanodevice. Even though usual low-power devices encompass SRAM memory in their structures $[16,44,46]$, due to the high bit density required in a nanodevice, novel DRAM-based technologies seem the best alternative to ensure a sufficient amount of memory -54 times higher than SRAM. However, the energy consumption of DRAM, which requires a continuous refresh signal, is its principal inconvenience. Combining the advantages of both types of RAM memories, the A-RAM is the technological alternative foreseeing high bit density and preserving restrained energy consumption [50]. Therefore, it will be the technology recommended for our RAM module.

\subsection{Nanoantenna}

Nanodevices, conceived as part of a wireless nanosensor network, require a full radiocommunication system to allow EM communication among them. Traditional patch antennas integrated into electronic devices, such as smartphones or laptops, are usually made of metallic 


\section{CHAPTER 1. CONCEPTUAL DESIGN OF A NANO-NETWORKING DEVICE}

materials. This is because metallic patch antennas (in the order) of few centimeters are able to radiate at the usual frequency range employed by most commercial technologies ( $\mathrm{GHz}$ frequencies). However, metallic antennas are not a feasible solution for a nanodevice since the radiation/resonant frequency of them for the nanoscale rises up to the range of hundreds of $\mathrm{THz}$ and, as a consequence, the channel attenuation at this frequency band would imply extremely poor transmission distances. To overcome this drawback, a different material is required to achieve lower radiation frequencies (units of terahertz) and, therefore, a restrained channel attenuation.

Graphene, a single-atom thick layer of carbon forming a honeycomb lattice, presents unique properties that have attracted the interest of the scientific community for creating a myriad of groundbreaking applications in many interesting disciplines [53-58]. However, the quality what makes graphene appropriate for nanoantennas is the ability to propagate surface-plasmon polaritons (SPP) waves [59]. Typically, SPP waves propagate enclosed in the interface between a metal and a dielectric layer. Noble metals, such as gold or silver, do support the propagation of SPP waves, but at higher frequencies than graphene. Furthermore, SPP waves on graphene exhibit additional advantages including easy tunability and low ohmic losses [60-62]. Under these conditions, graphene-based nanoantennas are envisaged to efficiently radiate electromagnetic waves in the range of 1 to $10 \mathrm{THz}$ [62-64], known as the terahertz band, which, according to the study in [65], involves resonant frequencies two orders of magnitude lower than those in a nanoscale metal antenna.

An additional carbon-based solution for a nanoantenna is the carbon nanotube (CNT). As was reported in [63], a nano-dipole based on a CNT and a graphene nanoribbon present similar radiation properties. Both nanoantennae (CNTs and nanoribbons) are able to radiate in the terahertz band for an antenna length equal to $1 \mu \mathrm{m}$. The resonant frequency of the nanoribbon is, however, slightly lower than that for the CNT, which is an advantage to achieve less propagation losses [66].

However, radiation frequency depends not only on the length of the nanoribbon. Additional parameters, such as the nanoribbon width, the type of substrate and its size, also influence the behavior of the nanoantenna. The width allows us to tune the frequency over a certain range. The nanoribbon resonates at a lower frequency as it becomes narrower [67]. If the antenna is wide enough, SPP waves change their characteristics and the graphene nanoantenna could radiate at frequencies similar to those in a metallic antenna. Therefore, the width must be sufficiently narrow to ensure the appropriate conditions for the SPP wave propagation.

On the other hand, the dielectric substrate employed to deposit the nanoantenna has an impact on the absorption cross-section, which measures the fraction of the incident power that is absorbed by the antenna. Thus, the resonant frequency corresponds to the frequency at which the absorption cross-section value is the maximum. The authors of [67] compared different substrate sizes. In detail, for a $5 \times 0.5 \mu \mathrm{m}$ (length $\times$ width) graphene ribbon placed at the center of the substrate, square substrates sizes varying from $6 \times 6 \mu \mathrm{m}$ to $16 \times 16 \mu \mathrm{m}$ were analyzed and discussed. The results showed that larger sizes reach higher absorption cross-section values, which involve a better antenna performance. The resonant frequency remains constant at $0.5 \mathrm{THz}$ as the substrate size changes. It is noteworthy that the substrate thickness for every simulation was set to $1 \mu \mathrm{m}$.

The dielectric material of the substrate also affects the nanoantenna absorption cross-section. A comparison among silicon, silicon dioxide $\left(\mathrm{SiO}_{2}\right)$ and vacuum for the same graphene nanoribbon was reported in [64]. This study observed that increasing the dielectric constant ( ) of the substrate, the peak of the absorption cross-section shifts to lower frequencies, whereas its value decreases. The outcome is a negative effect on the absorption efficiency. Thus, the substrate composed of $\mathrm{SiO}_{2}(=$ 4.0) achieved a two-fold increase of the absorption cross-section value in comparison with the silicon ( = 11.9), but at slightly higher frequencies.

Regarding the power required to radiate a signal in the terahertz band, a power spectral density value of $10^{-18} \mathrm{~W} / \mathrm{Hz}$ is considered in [68]. Integrating the spectral density over a bandwidth of $1 \mathrm{THz}$ (the expected bandwidth for the communication between nanodevices), the total emission power is 1 $\mu \mathrm{W}$. The estimated transmission distance is in the order of few millimeters for this radiation power [69], since propagation losses are huge in the terahertz band. Therefore, the nanoantenna is a 


\section{CHAPTER 1. CONCEPTUAL DESIGN OF A NANO-NETWORKING}

significant component in the nanodevice design since its power consumption is a limiting factor. Under this constraint, an ad-hoc communication protocol should be implemented to adjust its operation and keep the waste of energy within affordable bounds.

In addition, to complete the radiocommunication system, the nanodevice must integrate a terahertz signal generator to drive the antenna. Several studies have dealt with the development of novel terahertz transceivers, highlighting the graphene-based transceiver theoretically modeled and analyzed in [70]. There are two main advantages of this type of transceiver: (i) it is specifically designed for graphene nanoantennae, minimizing the losses due to impedance mismatch; and (ii) its tiny size, since the length to resonate in the terahertz band must be in the order of nanometers. Two principal blocks, the electric signal generator and the graphene-based plasmonic nano-transceiver compose the transceiver, as shown in Figure 4. To accomplish the transmission, the information bit stream to be sent modulates the electric signal (carrier), which is generated by the electric signal generator. The modulated signal is injected to the graphene-based plasmonic nano-transceiver, transforming the signal into an SPP wave. The SPP wave, which resonates in the terahertz band, reaches the antenna and is directly radiated to the space. In the reception stage, the behavior is the opposite. The nanoantenna receives the analog wave in the terahertz band. Then, the wave reaches the graphene-based plasmonic nano-transceiver that generates a digital signal, which ideally contains the original bit stream. This signal passes through a voltage regulator circuit, adapting the signal to the suitable voltage level, to be read by the nanoprocessor.

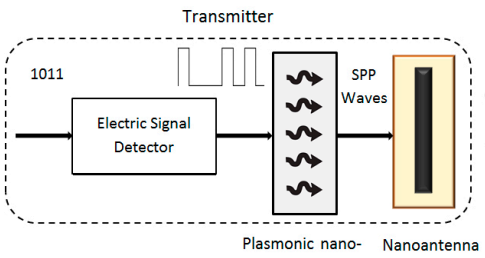

transceiver

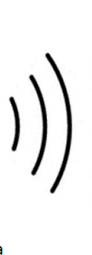$$
\text { (1) }
$$

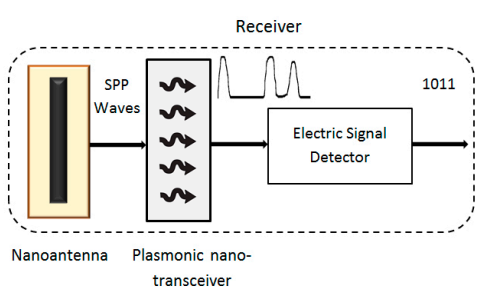

transceiver

Figure 4. Radiocommunication scheme, transmitter and receiver endpoints.

Technology Selection

As aforementioned, graphene is the best material for building a nanoantenna. A graphene nanoribbon and a CNT radiate in a similar fashion, with analogous radiation diagrams. However, in the case of a nanoribbon, the resonant frequencies are lower than those for a CNT nanodipole, which implies less propagation loss [65]. Regarding the nanodevice design, the nanoribbon can be integrated into the nanodevice easier than the CNT-based antenna, which would facilitate the nanodevice manufacture. Hence, we suggest the graphene nanoribbon as a reasonable technological solution for the nanoantenna design.

Additionally, the use of a substrate that improves the absorption efficiency of the antenna aids to increase its performance. This is an important issue due to the restricted transmission power. As was reviewed, $\mathrm{SiO}_{2}$ enhances the nanoantenna efficiency, so this material is seen as ap apropriate substrate candidate for our nanoantenna design.

As regards the nanotransceiver, the preferred solution is the graphene-based plasmonic nano-transceiver. As it employs the same material as the nanoantenna and follows the same principle to radiate in the terahertz band (using SPP waves), this nanotransceiver can be easily integrated into the nanodevice together with the proposed nanoantenna.

\subsection{Nanogenerator}

In the introduction, we proposed that as a general design requirement, our nanodevice size should be similar to the size of a blood cell. This tiny size makes it unfeasible to manipulate it to 


\section{CHAPTER 1. CONCEPTUAL DESIGN OF A NANO-NETWORKING}

replace a depleted battery. Thus, to guarantee an appropriate power level to feed the nanodevice efficiently, we consider two solutions: (i) harvesting energy from the environment (denoted as a self-powered nanodevice); and (ii) wireless energy induced from an external power source. Furthermore, we also propose the combination of both technologies (a hybrid solution), since the power provision is one of the major bottlenecks in the nanosensor networking field.

One of the most promising mechanisms to harvest energy from the environment is the use of zinc oxide $(\mathrm{ZnO})$ nanowires [71-74]. This technology is able to convert mechanical, vibrational or hydraulic energy into electric power due to the piezoelectric properties of the $\mathrm{ZnO}$. Specifically, this compound generates a positive voltage when it suffers a tensile strain-the material is stretched - whereas if the strain is compressive, the generated voltage is negative. Thus, when a $\mathrm{ZnO}$ nanowire bends by, for instance, a flow movement, one of its sides is stretched while the other is compressed simultaneously [75], generating a voltage difference along the nanowire (see Figure 5).

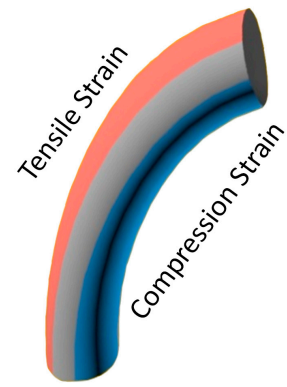

Figure 5. $\mathrm{ZnO}$ nanowire tensile and compression strains.

However, the electrical energy produced by just one nanowire is almost negligible. To produce enough energy to power our nanodevice, thousands of $\mathrm{ZnO}$ nanowires should be assembled into an array. The diameter for each nanowire ranges from 50 to $150 \mathrm{~nm}$ and its length is about $2 \mu \mathrm{m}$, which is suitable to be coupled with our nanodevice design. In order to enhance the nanogenerator performance, it is highly recommended to include a rectifying stage/circuit after the $\mathrm{ZnO}$ nanogenerator to adjust the voltage, since the potential difference can be positive or negative depending on the direction of the nanowires' movement. As evidence of the prospective of this technology, in [76], a self-powered electronic device is fed by a generator consisting of an array of $\mathrm{ZnO}$ nanowires vertically aligned. According to the authors, this layout is the most appropriate to attain the maximum performance of the generator.

Nanodevices are conceived to operate in different environments $[1,72,73]$, such as in the human body or in nature, where forces and vibrations occur in an irregular fashion, varying in frequency (mostly low frequencies) and magnitude. This negatively affects the effectiveness of the nanowires. Under these conditions, we can say that the power generated by a nanogenerator is intrinsically related to the environment under consideration and its behavior. Concerning the alignment of nanowires, the authors in [76] experimentally obtained the volumetric power density generated by an array of $\mathrm{ZnO}$ nanowires vertically aligned. This value, measured under laboratory conditions (supporting regular strains in frequency and intensity), was $0.01 \mathrm{pW} / \mu \mathrm{m}^{3}$, reaching voltage peaks of $10 \mathrm{~V}$. Even under ideal conditions, this result reveals that the amount of power produced by the nanogenerator is insufficient to feed the nanodevice continuously. To overcome it, the gathered energy must be stored in devices such as capacitors or batteries, intermittently powering the nanodevice when the charge level reaches a given value. Next, we analyze each of these storage technologies, condensing their main features in Table 4. 


\section{CHAPTER 1. CONCEPTUAL DESIGN OF A NANO-NETWORKING}

Table 4. Comparison among storing technologies.

\begin{tabular}{|c|c|c|c|}
\hline Storage Technology & Advantages & Disadvantages & Feasibility \\
\hline \multirow{3}{*}{ Batteries } & \multirow{3}{*}{ High energy density } & High degradation & \multirow{3}{*}{ Not clear } \\
\hline & & Mechanical properties & \\
\hline & & Use of toxic materials & \\
\hline \multirow{4}{*}{ Supercapacitors } & High capacitance & \multirow{4}{*}{ Low energy density } & \multirow{4}{*}{ Yes } \\
\hline & Ultra low degradation & & \\
\hline & $\begin{array}{l}\text { Mechanical properties } \\
\text { (flexible and thin) }\end{array}$ & & \\
\hline & Non-toxic materials & & \\
\hline
\end{tabular}

Batteries, as opposed to semiconductors, fail to comply with the Moore's law, since any known material can currently store a huge charge into an arbitrary small volume. Despite this, novel battery designs have been recently proposed [77-79] for the development of high-capacity nanobatteries, overcoming the scaling issues of traditional lithium ion batteries.

In this regard, the battery proposed in [77] is a solution composed by an array of nanobatteries connected in parallel, which will allow the implementation of prototypes to nanoscale. Each nanobattery contains two electrodes made of vanadium pentoxide $\left(\mathrm{V}_{2} \mathrm{O}_{5}\right)$ and a ruthenium $(\mathrm{Ru})$ nanotube acting as the current collector. The electrodes and Ru-nanotube are the principal components of a symmetric cell formed by the anode and cathode, in turn separated by a liquid electrolyte. This battery achieves a volumetric capacity of $147 \mathrm{mAh} \mathrm{g}^{-1}$ with a retention of $95 \%$ after 1000 charge/discharge cycles, and a duration of $12 \mathrm{~min}$. However, when the charge/discharge cycles become shorter ( $24 \mathrm{~s}$ ), the capacity retention drops to $46 \%$. This reduction (unused percentage after the charge/discharge cycles) is caused by the internal electrochemical process for storing energy, and supposes a physical change between the charge and the discharge states that slowly degrade its properties.

Other promising technology is described in [78], where the authors imitated the structure of a pomegranate to design a battery. This arrangement is composed of silicon particles encapsulated by a conductive carbon layer that enables enough space for running an expansion and contraction mechanism (charge and discharge process). The capacity retention achieved by this battery is $97 \%$ after 1000 charge/discharge cycles, but the cycle duration is not stated. In addition, the volumetric capacity is $1270 \mathrm{mAh} \mathrm{cm}^{-3}$, which ensures a suitable capacity for nanobatteries.

On the other hand, supercapacitors offer another form of carrying out the power storage, in a safe (aforementioned batteries usually use acid or toxic materials), durable and scalable fashion. A traditional capacitor consists of two metal plates separated by a thin insulating layer, where the charge is stored electrostatically. This method presents lower degradation than, for instance, the electrochemical process used in batteries. Thus, the amount of charge that can be stored in a capacitor depends on three aspects: (i) the capacitance of the dielectric material; (ii) the area of the metal plates; and (iii) the distance between them. Concerning the latest aspect, supercapacitors minimize this spacing by using a liquid electrolyte to separate both electrodes, increasing the capacitance per unit of area. Therefore, since the area in a nanodevice is hardly restricted, supercapacitors offer, a priori, a suitable solution towards reaching higher storage capacity than typical electrolytic capacitors.

One of the most promising supercapacitors was engineered in [80]. It is called a hybrid supercapacitor, since it integrates the features of the two following types of supercapacitors: (i) electric double-layer capacitors (EDLC); and (ii) pseudocapacitors. The main difference between them is the energy storage mechanism. EDLC implement a non-faradaic mechanism and no chemical oxidation-reduction (redox) reactions occur. In contrast, pseudocapacitors are based on Faradaic redox reactions, involving high energy electrode materials [81]. The energy densities achieved by using such electrode materials are higher than those in EDLC; however, the physical changes due to the faradaic reactions restrict their lifetime. Hybrid capacitors can achieve energy and power densities greater than EDLC without sacrificing the limited endurance of 


\section{CHAPTER 1. CONCEPTUAL DESIGN OF A NANO-NETWORKING DEVICE}

pseudocapacitors. This integration is feasible thanks to the employment of graphene doped with manganese dioxide $\left(\mathrm{MnO}_{2}\right)$. The energy density attained by this hybrid supercapacitor is up to six times larger than commercial carbon-based supercapacitors. Specifically, the volumetric capacitance provided by this hybrid supercapacitor is $1100 \mathrm{~F} / \mathrm{cm}^{3}$ and maintains $96 \%$ of the original storage capacity after 10,000 charge/discharge cycles-one order of magnitude higher than the batteries. Moreover, this supercapacitor exhibits a planar geometry (the typical structure consisting of two electrodes stacked vertically), is highly flexible, and can be folded without affecting its structural integrity.

As was previously mentioned, the main problem of harvesting energy is the variable behavior of the environment. If these conditions are not able to excite sufficiently the piezoelectric properties of the $\mathrm{ZnO}$ nanowires, the charge level could be lower than the one required by a nanodevice to be activated. Under these circumstances, fundamental nanodevice tasks such as processing, memory I/O or communications could not be performed. To face this drawback, we recommend an alternative power source, which should be incorporated into our design to supply "extra energy" to the nanodevice. Thereby, the nanodevice will not only depend on the energy harvested.

This requires the use of mechanical waves, such as ultrasounds, inducing vibrations in the $\mathrm{ZnO}$ nanowires and generating tensile-compressive strains. This is used in a promising mechanism to transfer power continuously as described in [82]. In addition, the same nanogenerator could be employed for both harvesting energy from the environment and gathering energy coming from an external source.

The authors in [83] analyzed different parameters to enhance the power transmission by ultrasounds. Two initial aspects must be taken into account. First, the nanogenerator employed in this work was made of $\mathrm{ZnO}$ nanowires. Second, the environment for the simulation was the human body, where the maximum ultrasound power density is limited by medical recommendations (720 $\mathrm{mW} / \mathrm{cm}^{2}$ ). On the other hand, the ultrasound frequency is also an important design parameter to improve the efficiency of the energy transmission. To this aim, two different frequency values were analyzed: $50 \mathrm{kHz}$ and $1 \mathrm{MHz}$. At low frequency, waves present lower attenuation in the human skin than at $1 \mathrm{MHz}$, which entails higher power transmission to the nanodevice. Moreover, the authors compared different nanowire densities, that is, the number of nanowires per unit of area. The outcome was that densities higher than 20 nanowires per $\mu \mathrm{m}^{2}$ clearly reduce their mobility, and, therefore, the energy absorbed by each one. Finally, authors also estimated the parameter known as conversion efficiency factor of the nanogenerator, comparing the input power (received by the nanowires) with respect to the output power (produced by the nanogenerator). The value of the conversion efficiency factor varies from $0.8 \%$ to $0.55 \%$, which could be improved by optimizing the nanowire fabrication technique (e.g., better nanowires alignment). The total power output density obtained from this work was $38.5 \mathrm{pW} / \mu \mathrm{m}^{2}$.

Technology Selection

As was previously described, powering the nanodevice by means of a ZnO-based (piezoelectric) nanogenerator is an outstanding solution provided that the environment allows it. Considering this primordial requirement, we have opted to power the nanodevice from two complementary energy sources. The first is a harvesting solution, which is feasible in an environment where a flow is in continuous movement, transmitting hydraulic energy to the $\mathrm{ZnO}$ nanowires (e.g., bloodstream). The second is an external source based on ultrasounds able to excite (tensile-compressive efforts) the nanowires constantly, intended to overcome the irregular behavior of the single harvesting solution. Once the energy sources are selected, we need to know their number, size and positioning to ensure a minimum operational availability of the nanodevice. Obviously, one single nanowire is unable to carry it out. Thus, a set of ZnO nanowires with a diameter of about $100 \mathrm{~nm}$ arranged in an array is the starting point to design the nanogenerator.

Other sources to generate electrical energy, such as chemical or heat energy, might be a suitable choice to power a nanodevice; however, as of today and to the best of our knowledge, this type of generator has not yet been developed at the nanoscale. 


\section{CHAPTER 1. CONCEPTUAL DESIGN OF A NANO-NETWORKING}

Regarding the power storage, in our opinion, the most current suitable technology to store the energy harvested is the hybrid supercapacitor. Even though electrochemical batteries can provide higher energy densities, their low efficiency and high degradation owing to the usual nanodevice operation with numerous charging and discharging cycles lead us to discard them. However, the mechanical features of hybrid supercapacitors (planar, flexible and durable) fit appropriately with our nanocapacitor design. In addition, their volumetric capacitance is, a priori, applicable to store sufficient energy and then to power the nanodevice intermittently, following the scheme used in $[72,76]$, where a device is fed by a capacitor storing the energy collected by a $\mathrm{ZnO}$ generator.

\section{Nanodevice Concept}

Once the technology for each component has been chosen, we conceptually outline the nanodevice. To this end, in the following subsections, we estimate the size of every component considering: (i) the overall dimensions of the nanodevice and its functionality; and (ii) the trade-off between the area dedicated to a component and the remaining size for the rest of the components. In relation to this latter aspect, an increase in the nanoprocessor area, for instance, has a noticeable impact on the performance of the rest of the components. Note that the component dimension affects its capabilities and, therefore, its functionality for the required envisaged tasks (monitoring, control, communications, etc.). The end result is shown in Figure 6, where our nanodevice layout is depicted, including the dimensions and technology selected for each component.

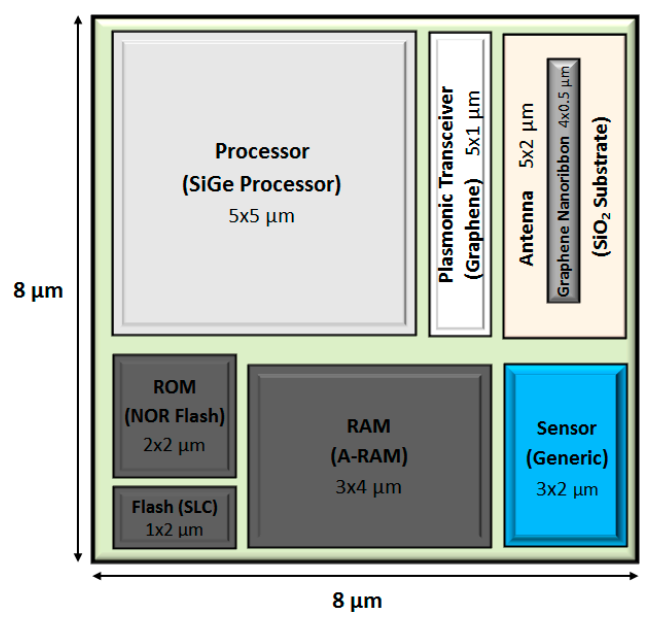

Figure 6. Nanodevice layout.

This layout is soundly conceived to favor the interconnection of components in the sense that it has been taken into consideration the trade-off between the components position in the overall area devoted to the nanodevice and their dimensions, as well as their specific capabilities and operation. The nanoprocessor is in charge of controlling the remaining elements; therefore, it is the core component taking more room. The memory modules (RAM, ROM and Flash) and the transceiver are located next to the nanoprocessor facilitating the information flow between these components. Then, the transceiver is also directly connected to the nanoantenna, in accordance with the radiocommunication scheme described above in Section 2.3. Lastly, the nanosensor is placed near to the RAM memory to store directly the data (which in turn will be managed by the nanoprocessor).

It should be noted that the sensor capabilities have not been tackled in this paper, because the integration of a specific sensor into a nanodevice directly depends on the application for which it is 


\section{CHAPTER 1. CONCEPTUAL DESIGN OF A NANO-NETWORKING DEVICE}

conceived. Therefore, the nanodevice layout described in this section has to be observed as a generalist hardware architecture able to support the basic tasks required to operate in a WNSN, regardless of the specific application. For that reason, the area reserved for the nanosensor $(3 \times 2 \mu \mathrm{m})$ in the layout has not been determined for a particular nanosensor, since many sensors could be placed on that area. For instance, the nanosensor described in [84], based on silicon nanowires (with an average width of $80 \mathrm{~nm}$ ) can detect $\mathrm{pH}$ changes in an aqueous medium, as well as alkali metallic ions ( $\mathrm{Na}$ and $\mathrm{K}$ ) concentrations. Other sensors based on $\mathrm{ZnO}$ nanowires are able to detect diverse parameters, such as pressure, mechanical strains or gas concentrations [85]. As reviewed in Section 2.4, the diameter for each $\mathrm{ZnO}$ nanowire ranges from 50 to $150 \mathrm{~nm}$ and its length is about $2 \mu \mathrm{m}$, which perfectly fits the area reserved in our layout. Also, sensors based on graphene nanowalls can be integrated into the nanodevice due to its great scalability features, as the ultrasensitive temperature sensor designed in [55].

\subsection{Nanoprocessor}

As described in Section 2, the single-transistor area for a SiGe technology chip is approximately $5000 \mathrm{~nm}^{2}$, so we will take this value as a reference in order to calculate the area of the future nanoprocessor. As a preliminary estimation, we propose to reserve a space into the nanodevice for the nanoprocessor of a square of $5 \times 5 \mu \mathrm{m}$, that is, a total area of $25 \mu \mathrm{m}^{2}$. Using these values, the total transistor count is $\approx 5000$ MOSFET, which is more than twice the number of transistors implemented, for instance, in an Intel 4004 processor and slightly more than the Intel 8080 [86]-the core of the Altair 8800 computer [87]. This amount of transistors assures a chip with enough computing capacity to run basic tasks such as read data from the sensor, memory write/read operations or manage a simple ad-hoc communication protocol. Therefore, the area allocated to the nanoprocessor is clearly suitable to operate the nanodevice and guarantee its functionality within a WNSN.

Another basic feature design for the nanoprocessor is the operational frequency. SiGe-based transistors are conceived to operate at higher frequencies than those made of commercial silicon. However, it entails a higher power consumption, which punishes the nanodevice functioning, since saving power is a critical concern. This leads us to consider it as a key requirement for the nanoprocessor to work at low frequencies. As stated in [44,88], a microprocessor working in the $\mathrm{kHz}$ range is able to collect and process sensing data for medical applications, also to switch between sleep and active modes to save energy nimbly. According to the specifications of the processors designed in these works, an appropriate value for the frequency of operation of the nanoprocessor can be established, a priori, around $500 \mathrm{kHz}$ to ensure the aforementioned basic tasks and reach acceptable performance.

SiGe technology chips will consume much less energy than their predecessors, the silicon-based solutions. In this sense, IBM quantifies this reduction by at least $50 \%$. To estimate the consumption of a SiGe-based nanoprocessor, we have taken as a reference the ultra-low-power processor, called Phoenix, designed and implemented in [16], which was mentioned in Section 2 as a reference in low power consumption $(2.8 \mathrm{nW} / \mathrm{kHz}$ at $0.5 \mathrm{~V})$. Following the specifications of this chip, all the components (CPU, ROM and RAM memories, timers and a temperature sensor among others) are included in a total area of $0.837 \mathrm{~mm}^{2}$ fabricated with a $0.18 \mu \mathrm{m}$ silicon technology. Thus, this processor size is around four orders of magnitude larger than our nanoprocessor proposal, so the power consumption also has to be scaled. Considering that (i) the nanoprocessor works at low frequency; (ii) novel technologies consume less energy in comparison with the silicon ones; and (iii) the scale effect-the nanoprocessor could consume $280 \mathrm{pW} / \mathrm{kHz}(0.28 \mathrm{pJ}$ per cycle), operating at 0.5 $\mathrm{V}$. This estimation also contemplates the ROM and RAM memories, since the Phoenix processor includes all of them. To keep the ultra-low energy consumption condition, the operation frequency is set to $500 \mathrm{kHz}$, thus the average power consumption of the nanoprocessor together with the ROM and RAM modules is $140 \mathrm{nW}$. 


\section{CHAPTER 1. CONCEPTUAL DESIGN OF A NANO-NETWORKING}

\subsection{Nanomemory}

The cell size for SLC NAND flash technology enabling write/read of a bit is about $784 \mathrm{~nm}^{2}$ (as justified in Section 2), so this will be our endorsed value to estimate the area devoted to the memory in the nanodevice.

As will be discussed in the following subsection (nanogenerator), the energy consumption for the SLC Flash memory ( $305 \mathrm{pJ} / \mathrm{bit}$ and $30 \mathrm{pJ} / \mathrm{bit}$ for the write and read operations, respectively) involves a truth bottleneck that restricts the amount of flash memory in our nanodevice. The reason is because the energy provided by the nanogenerator can only afford the writing of a small amount of flash memory bits. Therefore, under this limitation, we propose a flash memory module of 2048 bits (256 bytes), which is considered enough for storing specific information, such as the ID number assigned to the different nanodevices in the process of creating the network topology [89]. This amount of bits entails a total area of $1.61 \mu^{2}$ (2048 bits $\times 784 \mathrm{~nm}^{2}$ dedicated to each bit). Thus, we plan to assign a rectangle of $2 \times 1 \mu \mathrm{m}$ to make sure that the flash module can be integrated into the nanodevice layout.

The selected technology for the ROM module is the NOR flash memory, which has a cell area of $1293 \mathrm{~nm}^{2}$ consuming $0.2 \mathrm{pJ}$ per operation. We have projected a $2 \times 2 \mu \mathrm{m}$ rectangle for the ROM module, thus, having a total area of $4 \mu^{2}$. With these values, the ROM memory capacity is of 3093 bits ( $\approx 386$ bytes), which combined with a compact instruction set, as used in $[45,88]$, is sufficient to store the boot code.

Finally, the RAM module is placed in a rectangle of $3 \times 4 \mu \mathrm{m}$, with a total area of $12 \mu \mathrm{m}^{2}$. The cell size for A-RAM, which is envisaged to be of the same size as or even smaller than DRAM, is 1176 $\mathrm{nm}^{2}$, thus, RAM memory could store up to 10,204 bits ( $\approx 1275$ bytes). This space along with that assigned to the NAND flash module is enough to carry out basic monitoring applications, such as the process of measuring a physical variable (e.g., temperature) and to store its value in memory, as was reported in $[16,45]$

\subsection{Nanoantenna}

Concerning the nanodevice design, we must also decide the dimensions of the antenna to radiate in the selected frequency range. To this end, on the one hand, the nanoribbon length should be larger than its width to boost the SPP effect and, thus, radiate in the terahertz band. On the other hand, higher antenna length reaches larger channel capacity, as was reported in [69]. Therefore, to ensure an acceptable channel capacity, the dimensions that we have estimated for the graphene nanoribbon (considering the restrictions of the nanodevice size) are $4 \times 0.5 \mu \mathrm{m}$ (length $\times$ width). The substrate size estimated to allocate the antenna has a total size of $5 \times 2 \mu \mathrm{m}$. Larger substrate sizes would achieve better performance, but due to the limited area available in the nanodevice, a tight substrate size should be selected.

The thickness of the substrate is also a relevant requirement, since the third dimension of the nanodevice is hardly restricted. A substrate thickness of $1 \mu \mathrm{m}$ adapts well to our nanodevice layout and offers an appropriate performance in terms of absorption cross-section.

Considering the works in $[62,64,67]$, a substrate fabricated in $\mathrm{SiO}_{2}$ along with the dimensions selected for the graphene nanoribbon involve a radiation frequency around $1 \mathrm{THz}$. Therefore, as the bandwidth is directly related to the radiation frequency, the achieved throughput between devices in coverage can be extremely high, up to Tbits/s. However, due to the much lower nanoprocessor operational frequency $(500 \mathrm{kHz})$, nanodevices are not able to operate at these transmission rates. Thus, a transmission rate up to $500 \mathrm{Kbps}$ can be allocated in different frequency subbands of the $\mathrm{THz}$ spectrum aimed at, for instance, designing an ad-hoc communication protocol which, in networks formed by multiple nanodevices, was able to save energy (e.g., transmitting ultra-short pulses) or to avoid message/packet collisions, among others features.

To complete the radiocommunication system, the nanotransceiver should be allocated between the nanoprocessor and the nanoantenna. It includes the electric signal generator, which receives a bit stream from the nanoprocessor, and the plasmonic graphene-based transceiver, which drives the nanoantenna. The plasmonic transceiver length must be in the order of a hundred nanometers to 


\section{CHAPTER 1. CONCEPTUAL DESIGN OF A NANO-NETWORKING DEVICE}

generate waves in the terahertz band [70,90]. Thus, an area of $5 \times 1 \mu \mathrm{m}$ should be appropriate to place both components.

As regards the power consumption of the radiocommunication system, most of the energy will be employed on the emission power of the nanoantenna, since the nanotransceiver should be selected to minimize the losses between its own transceiver and the antenna. As was discussed in Section 2, an emission power on the order of $1 \mu \mathrm{W}$ satisfies an acceptable transmission range [70]; thus, we set this value of power consumption for the entire radiocommunication system.

\subsection{Nanogenerator}

In order to encapsulate all of the nanodevice components (memory, antenna, processor, etc.) in the same plane, it would seem reasonable to assume the placement of the nanogenerator and supercapacitor to be below the remaining components (see Figure 1). Using this arrangement, we can take advantage of the third dimension, as proposed in [1].

Therefore, in our layout, we consider the use of a supercapacitor to store the energy generated by the $\mathrm{ZnO}$ nanowires when they are bending. It should be placed below the circuit board and connected to the electrodes of the nanogenerator to be charged. Thus, the nanodevice will operate when the charge level exceeds a given value. In addition, to accumulate energy in the capacitor, the voltage and intensity should also be accurately adjusted at the level required by the different components of the nanodevice.

On the dimensions of the supercapacitor and the $\mathrm{ZnO}$ nanowires array, the aim is to employ the entire circuit board available area, that is, a square of $8 \times 8 \mu \mathrm{m}$ (length $\times$ width). The total available depth for both components is $3 \mu \mathrm{m}$ (in accordance with the blood cell dimension taken as the overall size of reference), divided into $1 \mu \mathrm{m}$ devoted for the supercapacitor, which is an affordable thickness according to [91], and $2 \mu \mathrm{m}$ for the $\mathrm{ZnO}$ nanogenerator. Since the volumetric capacitance reached by the selected supercapacitor is $1100 \mathrm{~F} / \mathrm{cm}^{3}$, the total capacitance for a volume of $64 \mu \mathrm{m}^{3}$ is $70.4 \mathrm{nF}$. Following with the nanogenerator design, another important feature is the number of $\mathrm{ZnO}$ nanowires. The authors in [83], suggested a nanowire density of 20 nanowires per $\mu \mathrm{m}^{2}$, which applied to our proposed layout, entails a total amount of $1280 \mathrm{ZnO}$ nanowires completely covering the nanodevice area.

To estimate the amount of energy that could be generated just by the movement, we take the blood flow as the working environment. This environment behaves in a more regular fashion than other scenarios (e.g., water quality in a river, atmospheric pollution measurement in the air, muscle movement, etc.). In a blood flow scenario, the strain intensity depends on the blood pressure and the frequency of the heart rate (pulse). Under these regular conditions, we can assess that the average power density generated is $0.01 \mathrm{pW} / \mu \mathrm{m}^{3}$. The nanogenerator volume is $128 \mu^{3}$, so the estimated average self-generated power is $1.28 \mathrm{pW}$ in accordance with the methodology found in [76]. Moreover, the output voltage has to be scaled down; a feasible value for the nanogenerator is $0.7 \mathrm{~V}$ [71], which entails a maximum energy stored in the supercapacitor of $17.24 \mathrm{~nJ}$, applying the well-known expression:

$$
E_{\max }=\frac{1}{2} C V_{g}^{2},
$$

where $C$ is the capacitance and $V_{g}$ is the voltage source. Conversely, we might transfer energy from an external source by using ultrasounds if the average power density generated by the nanogenerator is around $38.5 \mathrm{pW} / \mu \mathrm{m}^{2}$ [83]. Note that this value is obtained when emitting ultrasound waves at healthy power levels for humans. Thus, if the nanodevice were in another environment where the negative effect on humans could be considered negligible, higher power levels could be employed to supply additional energy to the nanodevice. Therefore, considering the available area, the total power transferred by ultrasounds is $2.46 \mathrm{nW}$, that is, three orders of magnitude higher than the self-generated power.

In view of the values for the energy consumption obtained for each component (as was described in previous sections and summarized in Table 5), we can estimate the total power consumed by the nanodevice. To calculate it, we adopt what we could consider as the "typical" 


\section{CHAPTER 1. CONCEPTUAL DESIGN OF A NANO-NETWORKING}

operation of a nanodevice in a WNSN, employing three different operating modes: (i) the sensing mode; (ii) the communication mode, and (iii) the sleep mode.

Table 5. Power consumption per component.

\begin{tabular}{cc}
\hline Component & Power Consumption (nW) \\
\hline Processor (ROM and RAM included) & 140 \\
Radiocommunication system & 1000 \\
Sensor & 50 \\
Flash memory module & $30 / 305^{1}$ \\
\hline
\end{tabular}

${ }^{1}$ Read/Write, given in $\mathrm{pJ} / \mathrm{bit}$.

Working in the sensing mode, the nanodevice performs sensing and processing tasks (the radiocommunication system is off). The communication mode encompasses the communications tasks, so all the components are active, with the exception of the sensor. Finally, the sleep mode deactivates the nanodevice, allowing the supercapacitor to be recharged. Notice that the flash memory module is used to store basic protocol parameters, so it will only be written during the network establishment. In this stage, the energy consumption is not a critical issue, since several charge cycles could be dedicated exclusively to this purpose. Thus, flash memory will not be read or written during the regular operation of the nanodevice, and is omitted from this energy consumption study.

Analyzing the data shown in Table 5, the total power consumed by our nanodevice during the sensing mode $\left(P_{s}\right)$ is $190 \mathrm{nW}$ and $1190 \mathrm{nW}$ operating in the communication mode $\left(P_{c o m}\right)$. The power difference between both modes reveals the important amount of energy consumed by the radiocommunication system, which is observed as the main bottleneck in the nanodevice operation.

As the power generated is lower than the power consumed in the sensing and communication cases, the nanodevice must work intermittently, alternating between active and sleep cycles to store energy. For instance, a typical communication task could be the transmission of an acquired temperature value (using for instance a typical payload of 2 bytes), encapsulated in a low-power protocol frame [92], employing a header of 19 bytes. Under these conditions, 21 bytes should be transmitted during a single active cycle. Assuming that one bit is dispatched in each nanoprocessor clock cycle $(2 \mu \mathrm{s})$, the transmission of a single data packet requires $336 \mu$ s. Observing the power consumed in the communication mode, the energy required to send one low-power protocol data packet is $0.4 \mathrm{~nJ}\left(E_{\text {total }}=P_{\text {com }} \times t_{\text {active }}\right)$, whereas the maximum energy stored in the supercapacitor, as was already calculated, is $17.24 \mathrm{~nJ}$. Therefore, the nanogenerator proposed should be able to handle the transmission of a typical information packet, which will be the most energy-demanding task.

Keeping in mind this operational scheme, the nanodevice could alternate among the three operating modes proposed to reduce the energy consumed and use the energy stored when necessary. Monitoring a physical measure, such as pressure or a hormone concentration, and sending the value when it exceeds a certain limit could be a suitable application for a nanodevice, which will require a fraction of the total energy stored in the supercapacitor to complete the communication.

\section{Conclusions}

A comprehensive conceptual design of a future nanoscale device employing current technology has been conducted. To this end, firstly, we analyzed the features/requirements of the main nanodevice components in depth, namely the nanoprocessor, nanomemory, nanoantenna-and its corresponding transceiver-and nanogenerator, for later integrating them into the nanodevice under consideration. For each component, we reviewed and discussed the pros and cons of the most usual and future technologies, paying special attention to the commercial and pre-commercial solutions, and selecting the most suitable one, according to its contribution to the nanodevice capabilities (e.g., sensing/acting, processing, memory, energy management, and telecommunication) and its size. Then, we discussed the feasibility of a nanodevice from a technological approach, 


\section{CHAPTER 1. CONCEPTUAL DESIGN OF A NANO-NETWORKING DEVICE}

selected to balance the ultra-restricted dimensions of the nanodevice (taken as the size of a blood cell), its functionality, and the energy consumption of the different components.

We humbly consider this work as a timely study proposing a viable nanodevice layout and a quantified technological solution. This will allow a grounded starting point in the emerging wireless nanosensor networking field, to further develop, for instance, ad-hoc communication protocols, but also to discover future applications or services in outstanding areas of research such as medicine (e.g., in the fight against cancer $[93,94]$ ) or environmental pollution (e.g., to help to combat climate change [95]), among others. This paper is seen as the foundations for our future work, and several aspects should be tackled. Firstly, a deeper discussion on the available fabrication techniques shall enlighten us about the technical barriers and feasibility to physically build a nanonetworking device, including the modules interconnection and integration issues. Then, the selection of specific sensors for particular applications is an open issue that will influence on refining and fine tuning our generalist nanodevice design. Finally, once the focus is on a given application, the WNSN should be adequately created, consisting of a huge number of nanonodes running cooperatively the communication protocols, and giving rise to a thorough performance evaluation study of their behavior.

Acknowledgments: This research was supported by the MINECO/FEDER Project Grants TEC2013-47016-C2-2-R (COINS) and TEC2016-76465-C2-1-R (AIM)

Author Contributions: Sebastian Canovas-Carrasco and Antonio-Javier Garcia Sanchez conceived the idea. Sebastian Canovas-Carrasco looked into the related literature. Antonio-Javier Garcia-Sanchez proposed the scientific methodology and made the 3D figures. Joan Garcia-Haro and Felipe Garcia-Sanchez contributed to the layout definition and technical improvement. All authors participated in the elaboration of the manuscript and its revision.

Conflicts of Interest: The authors declare no conflict of interest.

\section{References}

1. Akyildiz, I.F.; Jornet, J.M. Electromagnetic wireless nanosensor networks. Nano Commun. Netw. 2010, 1, 319.

2. Jornet, J.M.; Akyildiz, I.F. The internet of multimedia Nano-Things. Nano Commun. Netw. 2012, 3, $242-251$.

3. Akyildiz, I.F.; Jornet, J.M.; Han, C. Terahertz band: Next frontier for wireless communications. Phys. Commun. 2014, 12, 16-32.

4. Walsh, F. Protocols for Molecular Communication. Ph.D. Thesis, Waterford Institute of Technology, Waterford, Ireland, October 2013.

5. Wong, H.; Iwai, H. The road to miniaturization. Phys. World 2005, 18, 40-44.

6. ARM Holdings. ARM Cortex A-Series. Available online: http://www.arm.com/products/processors/cortex-a/index.php, Cambridge, United Kingdom (accessed on 9 December 2016).

7. Bangsaruntip, S.; Cohen, G.M.; Majumdar, A.; Sleight, J.W. Universality of short-channel effects in undoped-body silicon nanowire MOSFETs. IEEE Electron Device Lett. 2010, 31, 903-905.

8. Roy, K.; Mukhopadhyay, S.; Mahmoodi-Meimand, H. Leakage current mechanisms and leakage reduction techniques in deep-submicrometer CMOS circuits. Proc. IEEE 2003, 91, 305-327.

9. Franklin, A.D. Nanomaterials in transistors: From high-performance to thin-film applications. Science 2015, 349, doi:10.1126/science.aab2750.

10. Advancing Moore s Law-The Road to $14 \mathrm{~nm}$. Available online: http://www.intel.com/content/ www/us/en/silicon-innovations/advancing-moores-law-in-2014-presentation.html (accessed on 9 December 2016).

11. $16 \mathrm{~nm}$ Technology. Available online: http://www.tsmc.com/english/dedicatedFoundry/technology/ 16nm.htm (accessed on 9 December 2016)

12. Bohr, M. $14 \mathrm{~nm}$ Process Technology: Opening New Horizons. Intel 2014. Available online: http://www.intel.la/content/www/xl/es/architecture-and-technology/bohr-14nm-idf-2014-brief.html (accessed on 9 December 2016) 


\section{CHAPTER 1. CONCEPTUAL DESIGN OF A NANO-NETWORKING}

13. Huang, X.; Lee, W.; Kuo, C.; Hisamoto, D.; Chang, L.; Kedzierski, J.; Anderson, E.; Takeuchi, H.; Choi, Y.; Asano, K.; et al. Sub 50-nm FinFET: PMOS. In Proceedings of the International Electron Devices Meeting-Technical Digest, San Francisco, CA, USA, 5-8 December 1999.

14. Memsic LOTUS: High-Performance Wireless Sensor Network Platform-Datasheet. Available online: http://www.memsic.com/userfiles/files/Datasheets/WSN/6020-0705-01_A_LOTUS.pdf (accessed on 9 December 2016).

15. ARM Cortex-M0+ Characteristics. Available online: https://developer.arm.com/products/processors/ cortex-m/cortex-m0-plus (accessed on 9 December 2016).

16. Hanson, S.; Seok, M.; Lin, Y.; Foo, Z.; Kim, D.; Lee, Y.; Liu, N.; Sylvester, D.; Blaauw, D. A Low-Voltage Processor for Sensing Applications With Picowatt Standby Mode. IEEE J. Solid-State Circuits 2009, 44, 11451155

17. Del Alamo, J.A. Nanometre-scale electronics with III-V compound semiconductors. Nature 2011, 479, $317-$ 323

18. Lee, M.L.; Fitzgerald, E.A.; Bulsara, M.T.; Currie, M.T.; Lochtefeld, A. Strained Si, SiGe, and Ge channels for high-mobility metal-oxide-semiconductor field-effect transistors. J. Appl. Phys. 2005, 97, doi:10.1063/1.1819976.

19. Silicon Germanium Chips. Available online: http://www-03.ibm.com/ibm/history/ibm100/us/ en/icons/siliconchip/ (accessed on 9 December 2016).

20. International Business Machines. IBM Research Alliance Produces Industry s First $7 \mathrm{~nm}$ Node Test Chips. Available online: http://www-03.ibm.com/press/us/en/pressrelease/47301.wss (accessed on 9 December 2016)

21. Avouris, P.; Martel, M. Progress in Carbon Nanotube Electronics and Photonics. MRS Bull. 2010, 35, 306313.

22. Javey, A.; Guo, J.; Wang, Q.; Lundstrom, M.; Dai, H. Ballistic carbon nanotube field-effect transistors. Nature 2003, 424, 654-657.

23. Shulaker, M.M.; Van Rethy, J.; Wu, T.F.; Suriyasena Liyanage, L.; Wei, H.; Li, Z.; Pop, E.; Gielen, G.; Wong H.S.P.; Mitra, S. Carbon nanotube circuit integration up to sub-20 nm channel lengths. ACS Nano 2014, 8 , 3434-3443.

24. Shulaker, M.M.; Hills, G.; Patil, N.; Wei, H.; Chen, H.Y.; Wong, H.S.; Mitra, S. Carbon nanotube computer. Nature 2013, 501, 526-530

25. Schulz, M. The end of the road for silicon? Nature 1999, 399, 729-730.

26. Appenzeller, J. Carbon nanotubes for high-performance electronics-Progress and prospect. Proc. IEEE 2008, 96, 201-211.

27. Cao, Q.; Han, S.-J.; Tersoff, J.; Franklin, A.D.; Zhu, Y.; Zhang, Z.; Tulevski, G.S.; Tang, J.; Haensch, W. End-bonded contacts for carbon nanotube transistors with low, size-independent resistance. Science 2015 $350,68-72$.

28. Lu, W.; Xie, P.; Lieber, C.M. Nanowire transistor performance limits and applications. IEEE Trans. Electron Devices 2008, 55, 2859-2876.

29. Iwai, H. Roadmap for $22 \mathrm{~nm}$ and beyond. Microelectron. Eng. 2009, 86, 1520-1528.

30. Park, H.; Afzali, A.; Han, S.-J.; Tulevski, G.S.; Franklin, A.D.; Tersoff, J.; Hannon, J.B.; Haensch, W. High-density integration of carbon nanotubes via chemical self-assembly. Nat. Nanotechnol. 2012, 7, 787791.

31. Fuechsle, M.; Miwa, J.A.; Mahapatra, S.; Ryu, H.; Lee, S.; Warschkow, O.; Hollenberg, L.C.L.; Klimeck, G.; Simmons, M.Y. A single-atom transistor. Nat. Nanotechnol. 2012, 7, 242-246.

32. Pavan, P.; Bez, R.; Olivo, P.; Zanoni, E. Flash memory cells - an overview. Proc. IEEE 1997, 85, 1248-1271.

33. Mohan, V.; Bunker, T.; Grupp, L.; Gurumurthi, S.; Stan, M.R.; Swanson, S. Modeling power consumption of NAND flash memories using flashpower. IEEE Trans. Comput. Des. Integr. Circuits Syst. 2013, 32, 10311044

34. Aritome, S.; Kikkawa, T. Scaling challenge of self-aligned STI cell (SA-STI cell) for NAND flash memories. Solid. State. Electron. 2013, 82, 54-62.

35. Kadowaki, T.; Yamakawa, Y.; Nakamura, H.; Kimura, Y.; Niwano, M.; Masuoka, F. A new architecture for high-density high-performance SGT nor flash memory. IEEE Trans. Circuits Syst. II Express Briefs 2008, 55, $551-555$. 


\section{CHAPTER 1. CONCEPTUAL DESIGN OF A NANO-NETWORKING DEVICE}

36. Samsung NAND Basics. Available online: http://www.samsung.com/global/business/semiconductor/ minisite/SSD/M2M/html/whitepaper/whitepaper03.html (accessed on 9 December 2016).

37. Tanaka, T.; Helm, M.; Vali, T.; Ghodsi, R.; Kawai, K.; Park, J.K.; Yamada, S.; Pan, F.; Einaga, Y.; Ghalam, A.; et al. A 768Gb 3b/cell 3D-floating-gate NAND flash memory. In Proceedings of the 2016 IEEE International Solid-State Circuits Conference (ISSCC), San Francisco, CA, USA, 31 January-4 February 2016.

38. Verma, V.; Taneja, S.; Singh, P.; Jain, S.K. A 128-kb 10\% power reduced $1 \mathrm{~T}$ high density ROM with $0.56 \mathrm{~ns}$ access time using bitline edge sensing in sub $16 \mathrm{~nm}$ bulk FinFET technology. In Proceedings of the 28th IEEE International System-on-Chip Conference (SOCC), Beijing, China, 8-11 September 2015.

39. Parkin, S.S.P.; Hayashi, M.; Thomas, L. Magnetic Domain-Wall Racetrack Memory. Science 2008, 320, 190-194.

40. Seok, M.; Hanson, S.; Seo, J.S.; Sylvester, D.; Blaauw, D. Robust ultra-low voltage ROM design. In Proceedings of the 2008 IEEE Custom Integrated Circuits Conference, San Jose, CA, USA, 21-24 September 2008.

41. Zhao, W.S.; Zhang, Y.; Trinh, H.-P.; Klein, J.-O.; Chappert, C.; Mantovan, R.; Lamperti, A.; Cowburn, R.P Trypiniotis, T.; Klaui, M.; et al. Magnetic domain-wall racetrack memory for high density and fast data storage. In Proceedings of the IEEE 11th International Conference on Solid-State and Integrated Circuit Technology, Xian, China, 29 October-1 November 2012.

42. Ranmuthu, K.T.M.; Pohm, A.V.; Daughton, J.M.; Comstock, C.S. New low current memory modes with giant magneto-resistance materials. IEEE Trans. Magn. 1993, 29, 2593-2595.

43. Venkataraman, K.; Moodera, J.S. High Density Molecular Memory Storage with Read and Write Capabilities. U.S. Patent 20,130,100,724, April 2013.

44. Sridhara, S.R.; DiRenzo, M.; Lingam, S.; Lee, S.J.; Blázquez, R.; Maxey, J.; Ghanem, S.; Lee, Y.H.; Abdallah, R.; Singh, P.; et al. Microwatt embedded processor platform for medical system-on-chip applications. IEEE J. Solid-State Circuits 2011, 46, 721-730.

45. Zhai, B.; Pant, S.; Nazhandali, L.; Hanson, S.; Olson, J.; Reeves, A.; Minuth, M.; Helfand, R.; Austin, T. Sylvester, D.; Blaauw, D. Energy-efficient subthreshold processor design. IEEE Trans. VLSI Syst. 2009, 17, $1127-1137$.

46. Kwong, J.; Ramadass, Y.; Verma, N.; Koesler, M.; Huber, K.; Moormann, H.; Chandrakasan, A. A 65nm Sub-Vt microcontroller with integrated SRAM and switched-capacitor DC-DC converter. In Proceedings of the 2008 IEEE International Solid-State Circuits Conference-Digest of Technical Papers, San Francisco, CA, USA, 3-7 February 2008.

47. ITRS Roadmap 2011. Available online: http://www.itrs2.net/ (accessed on 9 December 2016)

48. Ramani, A.R.; Choi, K. A novel 9T SRAM design in sub-threshold region. In Proceedings of the 2011 IEEE International Conference on Electro/Information Technology, Mankato, MN, USA, 15-17 May 2011.

49. Song, T.; Rim, W.; Jung, J.; Yang, G.; Park, J.; Park, S.; Baek, K.-H.; Baek, S.; Oh, S.-K.; Jung, J.; et al. A 14 nm FinFET 128Mb 6T SRAM with Vmin-enhancement techniques for low-power applications. In Proceedings of the 2014 IEEE International Solid-State Circuits Conference Digest of Technical Papers (ISSCC), San Francisco, CA, USA, 9-13 February 2014.

50. Rodriguez, N.; Gamiz, F.; Cristoloveanu, S. A-RAM memory cell: Concept and operation. IEEE Electron Device Lett. 2010, 31, 972-974.

51. Yang, H.Y.; Chang, C.M.; Chao, M.C.T.; Huang, R.F.; Lin, S.C. Testing methodology of embedded DRAMs. IEEE Trans. VLSI Syst. 2012, 20, 1715-1728.

52. Kim, I.S.; Cho, S.L.; Im, D.H.; Cho, E.H.; Kim, D.H.; Oh, G.H.; Ahn, D.H.; Park, S.O.; Nam, S.W.; Moon, J.T.; et al. High performance PRAM cell scalable to sub-20nm technology with below $4 \mathrm{~F}^{2}$ cell size, extendable to DRAM applications. In Proceedings of the 2010 Symposium on VLSI Technology, Honolulu, HI, USA, 15-17 June 2010

53. Avouris, P.; Xia, F. Graphene applications in electronics and photonics. MRS Bull. 2012, 37, 1225-1234.

54. Shahil, K.M.F.; Balandin, A.A. Thermal properties of graphene and multilayer graphene: Applications in thermal interface materials. Solid State Commun. 2012, 152, 1331-1340.

55. Yang, J.; Wei, D.; Tang, L.; Song, X.; Luo, W.; Chu, J.; Gao, T.; Shi, H.; Du, C. Wearable temperature sensor based on graphene nanowalls. RSC Adv. 2015, 5, 25609-25615.

56. Sorkin, V.; Zhang, Y.W. Graphene-based pressure nano-sensors. J. Mol. Model. 2011, 17, 2825-2830.

57. Fowler, J.D.; Allen, M.J.; Tung, V.C.; Yang, Y.; Kaner, R.B.; Weiller, B.H. Practical chemical sensors from chemically derived graphene. ACS Nano 2009, 3, 301-306. 


\section{CHAPTER 1. CONCEPTUAL DESIGN OF A NANO-NETWORKING}

58. Rao, F.; Fan, Z.; Dong, L.; Li, W. Molecular nanosensors based on the inter-sheet tunneling effect of a bilayer graphene. In Proceedings of the 2010 IEEE International Conference on Nano/Molecular Medicine and Engineering, Jeju, Korea, 9-12 November 2011.

59. Hanson, G.W. Dyadic green s functions for an anisotropic, non-local model of biased graphene. IEEE Trans. Antennas Propag. 2008, 56, 747-757.

60. Koppens, F.H.L.; Chang, D.E.; García de Abajo, F.J. Graphene Plasmonics: A Platform for Strong LightMatter Interactions. Nano Lett. 2011, 11, 3370-3377.

61. Gómez-Díaz, J.S.; Perruisseau-Carrier, J. Graphene-based plasmonic switches at near infrared frequencies. Opt. Express 2013, 21, 15490-15504.

62. Jornet, J.M.; Akyildiz, I.F. Graphene-based Plasmonic Nano-Antenna for Terahertz Band Communication in Nanonetworks. IEEE J. Sel. Areas Commun. 2013, 31, 685-694.

63. Jornet, J.M.; Akyildiz, I.F. Graphene-based nano-antennas for electromagnetic nanocommunications in the terahertz band. In Proceedings of the 4th European Conference on Antennas and Propagation (EuCAP), Barcelona, Spain, 12-16 April 2010.

64. Llatser, I.; Kremers, C.; Cabellos-Aparicio, A.; Jornet, J.M.; Alarcón, E.; Chigrin, D.N. Graphene-based nano-patch antenna for terahertz radiation. Photonics Nanostruct. Fundam. Appl. 2012, 10, 353-358.

65. Llatser, I.; Kremers, C.; Cabellos-Aparicio, A.; Alarcón, E.; Chigrin, D.N. Comparison of the resonant frequency in graphene and metallic nano-antennas. In Proceedings of the Fifth International Workshop on Theoretical and Computational Nano-Photonics: TaCoNa-Photonics, Bad Honnef, Germany, 24-26 October 2012.

66. Boronin, P.; Petrov, V.; Moltchanov, D.; Koucheryavy, Y.; Jornet, J.M. Capacity and throughput analysis of nanoscale machine communication through transparency windows in the terahertz band. Nano Commun. Netw. 2014, 5, 72-82.

67. Llatser, I.; Kremers, C.; Chigrin, D.N.; Jornet, J.M.; Lemme, M.C.; Cabellos-Aparicio, A.; Alarcon, E. Characterization of graphene-based nano-antennas in the terahertz band. In Proceedings of the 6th European Conference on Antennas and Propagation (EUCAP), Prague, Czech Republic, 26-30 March 2012.

68. Cabellos-Aparicio, A.; Llatser, I.; Alarcon, E.; Hsu, A.; Palacios, T. Use of Terahertz Photoconductive Sources to Characterize Tunable Graphene RF Plasmonic Antennas. IEEE Trans. Nanotechnol. 2015, 14, 390 396.

69. Llatser, I.; Cabellos-Aparicio, A.; Alarcon, E.; Jornet, J.M.; Mestres, A.; Lee, H.; Sole-Pareta, J. Scalability of the channel capacity in graphene-enabled wireless communications to the nanoscale. IEEE Trans. Commun. 2015, 63, 324-333.

70. Jornet, J.M.; Akyildiz, I.F. Graphene-based plasmonic nano-transceiver for terahertz band communication. In Proceedings of the 8th European Conference on Antennas and Propagation (EuCAP 2014), Hague, The Netherlands, 6-11 April 2014.

71. Wang, Z.L.; Song, J. Piezoelectric Nanogenerators Based on Zinc Oxide Nanowire Arrays. Science 2006, $312,242-246$.

72. Wang, Z.L. Self-Powered Nanotech. Sci. Am. 2008, 298, 82-87.

73. Wang, Z.L. Self-powered nanosensors and nanosystems. Adv. Mater. 2012, 24, 280-285.

74. Xu, S.; Qin, Y.; Xu, C.; Wei, Y.; Yang, R.; Wang, Z.L. Self-powered nanowire devices. Nat. Nanotechnol. 2010, 5, 366-373.

75. Wang, Z.L. Towards self-powered nanosystems: From nanogenerators to nanopiezotronics. Adv. Funct. Mater. 2008, 18, 3553-3567.

76. Hu, Y.; Zhang, Y.; Lin, C.X.L.L.; Snyder, R.L.; Wang, Z.L. Self-Powered System with Wireless Data Transmission. Nano Lett. 2011, 11, 2572-2577.

77. Liu, C.; Gillette, E.I.; Chen, X.; Pearse, A.J.; Kozen, A.C.; Schroeder, M.A.; Gregorczyk, K.E.; Lee, S.B Rubloff, G.W. An all-in-one nanopore battery array. Nat. Nanotechnol. 2014, 9, 1031-1039.

78. Liu, N.; Lu, Z.; Zhao, J.; McDowell, M.T.; Lee, H.-W.; Zhao, W.; Cui, Y. A pomegranate-inspired nanoscale design for large-volume-change lithium battery anodes. Nat. Nanotechnol. 2014, 9, 187-192.

79. Ji, L.; Tan, Z.; Kuykendall, T.; An, E.J.; Fu, Y.; Battaglia, V.; Zhang, Y. Multilayer nanoassembly of Sn-nanopillar arrays sandwiched between graphene layers for high-capacity lithium storage. Energy Environ. Sci. 2011, 4, 3611-3616. 
CHAPTER 1. CONCEPTUAL DESIGN OF A NANO-NETWORKING DEVICE

80. El-Kady, M.F.; Ihns, M.; Li, M.; Hwang, J.Y.; Mousavi, M.F.; Chaney, L.; Lech, A.T.; Kaner, R.B. Engineering three-dimensional hybrid supercapacitors and microsupercapacitors for high-performance integrated energy storage. Proc. Natl. Acad. Sci. USA 2015, 112, 4233-4238.

81. Stoller, M.D.; Park, S.; Zhu, Y.; An, J.; Ruoff, R.S. Graphene-Based Ultracapacitors. Nanto Lett. 2008, 8, 34983502 .

82. Wang, X.; Song, J.; Liu, J.; Wang, Z.L. Direct-Current Nanogenerator Driven by Ultrasonic Waves. Science 2007, 316, 102-105.

83. Donohoe, M.; Balasubramaniam, S.; Jennings, B.; Jornet, J.M. Powering In-Body Nanosensors with Ultrasounds. IEEE Trans. Nanotechnol. 2016, 15, 151-154.

84. Park, I; Li, Z; Pisano, A.P.; Williams, R.S. Top-down fabricated silicon nanowire sensors for real-time chemical detection. Nanotechnology 2010, 21, doi:10.1088/0957-4484/21/1/015501.

85. Shehada, N.; Brönstrup, G.; Funka, K.; Christiansen, S.; Leja, M.; Haick, H. Ultrasensitive silicon nanowire for real-world gas sensing: Noninvasive diagnosis of cancer from breath volatolome. Nano Lett. 2015, 15, 1288-1295.

86. Transistors to Transformations Available online: http://exploreintel.com/assets/pdf/aloha/museum-transistors-to-transformations-brochure.pdf (accessed on 9 December 2016).

87. Altair 8800 Microcomputer. Available online: http://americanhistory.si.edu/collections/search/ object/nmah_334396 (accessed on 9 December 2016).

88. Seok, M.; Hanson, S.; Lin, Y.S.; Foo, Z.; Kim, D.; Lee, Y.; Liu, N.; Sylvester, D.; Blaauw, D. The phoenix processor: A $30 \mathrm{pW}$ platform for sensor applications. In Proceedings of the 2008 IEEE Symposium on VLSI Circuits, Honolulu, HI, USA, 18-20 June 2008.

89. Rodenas-Herraiz, D.; Garcia-Sanchez, A.-J.; Garcia-Sanchez, F.; Garcia-Haro, J. On the improvement of wireless mesh sensor network performance under hidden terminal problems. Future Gener. Comput. Syst. $2015,45,95-113$.

90. Knap, W.; Teppe, F.; Dyakonova, N.; Coquillat, D.; Lusakowski, J. Plasma wave oscillations in nanometer field effect transistors for terahertz detection and emission. J. Phys. Condens. Matter 2008, 20, doi:10.1088/0953-8984/20/38/384205.

91. El-Kady, M.F.; Strong, V.; Dubin, S.; Kaner, R.B. Laser Scribing of High-Performance and Flexible Graphene-Based Electrochemical Capacitors. Science 2012, 335, 1326-1330.

92. IEEE Std $802.15 .4 ; 2011$. Available online: https://standards.ieee.org/findstds/standard/802.15.4-2011.html (accessed on 9 December 2016)

93. A New Superweapon in the Fight against Cancer. Available online: https://hammondlab.mit.edu/news/ new-superweapon- fight-against-cancer (accessed on 9 December 2016).

94. Kosaka, P.M.; Pini, V.; Ruz, J.J.; da Silva, R.A.; González, M.U.; Ramos, D.; Calleja, M.; Tamayo, J. Detection of cancer biomarkers in serum using a hybrid mechanical and optoplasmonic nanosensor. Nat. Nanotechnol. 2014, 9, 1047-53.

95. 7 Ways Nanotechnology Could Provide The Solutions To Combat Climate Change. Available online: http://www.worldnanofoundation.com/blog/paris-climate-summit-2016-nanotechnology-solutions-7 (accessed on 9 December 2016).

(c) 2016 by the authors. Submitted for possible open access publication under the terms and conditions of the Creative Commons Attribution (CC-BY) license (http://creativecommons.org/licenses/by/4.0/) 

Chapter 2

The IEEE 1906.1 standard: Nanocommunications as a new source of data 
CHAPTER 2. THE IEEE 1906.1 STANDARD: NANOCOM. AS A NEW

\section{THE IEEE 1906.1 STANDARD: NANOCOMMUNICATIONS AS A NEW SOURCE OF DATA}

Sebastian Canovas-Carrasco, Antonio-Javier Garcia-Sanchez, Joan Garcia-Haro

Department of Information and Communication Technologies, Technical University of Cartagena, Cartagena, Spain.

Emails: sebas.canovas@upct.es, antoniojavier.garcia@upct.es, joang.haro@upct.es

\section{ABSTRACT}

Nanoscale communications is a new paradigm encompassing all those concerns related to the exchange of information among devices at the nanometer scale. A network infrastructure consisting of a huge amount of nano-devices is envisaged to ensure robust, reliable and coordinated data transmission. This will enable a plethora of forthcoming applications and services in many differen research fields, such as personalized medicine, synthetic biology, environmental science or industry, which will lead to outstanding and unprecedented advances. The IEEE P1906.1 standard provides a conceptual and general framework to set the starting point for future developments in nanoscale communication networks. This paper reviews the latest IEEE P1906.1 recommendations, observing their main features when applied to the electromagnetic (EM) nanocommunication area. We contribute by identifying and discussing the principal shortcomings of the standard, to which further research efforts must be devoted. We also provide interesting guidelines for focusing the object of future investigations.

Keywords - Nanoscale communication networks, nanodevices, EM nanocommunications, terahertz band, IEEE standards

\section{INTRODUCTION}

In the emerging Internet of Things (IoT), objects are expected to be able to sense and capture the physical variables of their surroundings (e g temperature, humidity, pressure, etc ) as well as to process the acquired information and communicate it wirelessly to any other object/node in their network These enhanced objects integrate small sensing/computing/communicating devices in a varied range of sizes, including the nanoscale Moreover, devices in Io' constitute a network infrastructure connecting both physical and virtual worlds by means of all sorts of innovative applications and services, some of them currently unimaginable In this context, a huge amount of data will be generated and should be properly managed to extract useful

This work has been supported by the project AIM, ref

TEC2016-76465-C2-1-R (AEI/FEDER, UE) information Nowadays, the IoT relies on the well-known Wireless Sensor Networks (WSN), in which numerous devices with limited resources are connected, in order to provide feasible solutions in multiple heterogeneous fields, such as agriculture, industry, smart cities, etc Keeping in mind the way WSN operate and due to incessant technological advances, novel devices with progressively smaller dimensions are being developed, to ease their integration into the environment However, as they become smaller, many concerns, such as available energy, transmission range or data processing capacity are far more restricted than in traditional WSN Thus, when the scale of these tiny devices decreases to nanometers, a new paradigm arises, nanoscale communications between nanomachines, and between nanomachines and more conventional devices in the network

These data-driven nanodevices have become a topic of increasing interest for the scientific community, since they would be able to gather physical parameters at the nanoscale with outstanding accuracy This capacity would allow the monitoring of scenarios not explored to date, enabling a plethora of potential applications in fields as varied as biomedicine, synthetic biology, environmental science or industry, among many others Indeed, one of the most promising applications of these nanodevices is aimed at improving medicine, because diverse medical tests, such as blood pressure, virus detection or oxygen levels in blood (Figure 1), could be collected in vivo and directly transmitted to medical personnel (e g information about the variation in number and size of cancer cells will be received by the oncologist)

Several works have dealt with how nanodevices should communicate with each other This is becoming a critical issue, since the extremely limited resources of nanodevice require them to work cooperatively to carry out a useful application Two main alternatives for communicating at the nanoscale have been envisaged so far, electromagnetic (EM) and molecular communication

EM communication is based on the use of electromagnetic waves to transmit a message between two nanodevices Advancements in carbon electronics, mainly those devices made of graphene and carbon nanotubes (CNT), have played a key role in the development of a new generation of electronic nanocomponents, such as nanoantennas or 
CHAPTER 2. THE IEEE 1906.1 STANDARD: NANOCOM. AS A NEW

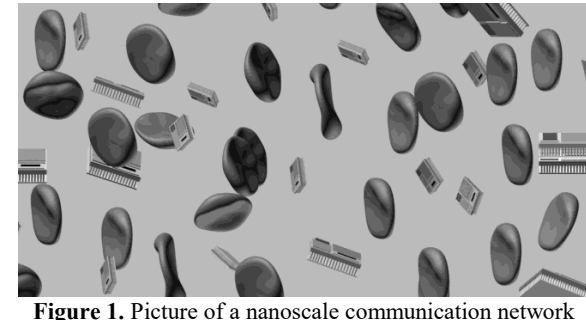

Figure 1. Picture of a nanoscale communication network deployed in the bloodstream

nanotransceivers [1]-[3] These novel radiocommunication nanocomponents possess unbeatable properties, which allow the radiation of EM waves at $\mathrm{THz}$ frequencies with antennas of just a few micrometers in length, i e two orders of magnitude lower than their metallic counterparts Even so, this radiation frequency exhibits high propagation losses, which require a thorough nanoscale communication network design, also known as a nanonetwork On the othe hand, molecular communication is defined as the transmission and reception of information encoded in organic molecules [4], [5] Molecular transceivers are envisioned to facilitate their integration into nanodevice due to their extremely small size and limited domain of operation These transceivers can react when receiving certain molecules and release others (as a response to stimulation or after executing some process) The molecules transmitted are propagated in three different ways: moving through a fluidic medium by free diffusion (diffusionbased); moving through a fluidic medium with a guided flow (flow-based); or through pre-defined pathways by using carrier substances (walkway-based)

Both EM and molecular, nanocommunications are considered by the IEEE P1906 1 standard; the firs approach to normalize diverse aspects related to communications at the nanoscale, released in December 2015 Under this general premise, this standard first defines the concept of a nanoscale communication network itself, to later propose a conceptual framework for developing communications Studies using the guidelines of this standard would implement a similar protocol stack for each nanodevice; it is recommended that this stack be based on the components and procedures specified by the IEEE P1906 1 to share and compare results from a common set of performance metrics as defined by the standard

This paper reviews the IEEE P1906 1 standard, focusing on EM communications; an area in which remarkable technological advances are leading to the first realistic approaches at the nanoscale In particular, we analyze the standard definition, its pros and cons, describe the framework offered along with its components and, finally, introduce the main metrics which will be taken into consideration to evaluate the performance of a nanoscale communication network Furthermore, we provide a functional EM communication scheme in which all the steps required to send/receive a message between a transmitter/receiver pair are explained in detail Analyzing the standard completely, we have identified some lacks and weaknesses, which are further addressed and discussed in this work These shortcomings pose important challenges A few of them have been dealt with in previous works [6]-[9], but most of them are still unexplored, which will undoubtedly be the starting point for future investigation

The rest of the paper is organized as follows In section 2, we review the IEEE P1906 1 standard from the perspective of EM communications Section 3 is devoted to pointing out some weaknesses of the standard for the design of EM nanonetworks In section 4, we indicate how to tackle each detected weakness Section 5 concludes the paper

\section{IEEE P1906.1 STANDARD DESCRIPTION UNDER EM COMMUNICATIONS}

As interdisciplinary research groups are becoming more and more involved in the development of nanoscale communications, the lack of a clear common scope has been confirmed, leading to isolated developments and unrelated knowledge islands In this sense, the different cases of study proposed in the open scientific literature have been thought about and evaluated under very specific conditions, which differ for each work This negatively impacts the exchange of information at the nanoscale, since nanonetwork performance depends on the particular working conditions and capabilities of nanodevices Thus, the IEEE P1906 1 standard [10] is aimed at providing a common framework, in order to join efforts and promote future advances in nanoscale communications In addition, another significan contribution of this standard is allowing sufficient precision for the development of interoperable and reusable components To achieve these goals, we examine the following four aspects of the standard structure: (i) definitions, (ii) framework, (iii) metrics, and (iv) EM communication reference model

\subsection{Definitions}

The first part of the standard provides a complete and detailed definition of "nanoscale communication network", which should pave the way for future studies in this emerging research field This definition intends to strictly establish the scope of this concept but, keeping it general enough to cover both molecular and EM communications The range chosen to delimit the nanoscale is quite narrow (from $1 \mathrm{~nm}$ to $100 \mathrm{~nm}$ ), extracted from the definition of nanoscale provided in [11] The lower limit is simply selected to exclude the use of single atoms as nanoscale systems In contrast, the upper limit is the size at which material properties change substantially from the macroscale This limit could cause controversy, since most of the scientific papers related to EM nanoscale communications consider nanodevices at larger scales Nevertheless, the sentence "at or with the nanoscale" contained in the definition leaves the door open to different considerations, in particular, those concerning the size of 


\section{CHAPTER 2. THE IEEE 1906.1 STANDARD: NANOCOM. AS A NEW}

the nanoscale object under study Therefore, the nanodevices proposed in these papers would support the standard whenever they include a communication element at the nanoscale These communication elements (transmitter, receiver, medium, message, and message carrier) are also named in the definition, even though they are not described in detail

\subsection{Framework}

The second block defined by the standard provides a conceptual, general and small-scale framework consisting of an appropriate number of components comprising welldefined functions and with interoperability among them The framework offers the organization and structure required to implement procedures and models To this end a set of interconnecting components is introduced, namely: (i) message carrier, (ii) motion, (iii) field, (iv) perturbation, and (v) specificity

The message carrier is described as the physical entity which transports the message across the medium In particular, in EM nanocommunications, message carrie would indicate the EM wave The motion componen represents the physical phenomenon that enables the message carrier to move (in EM, the wave propagation and phase velocity) This component may be randomly propagated through the medium, which would hamper the propagation of the wave To avoid this concern, the field component organizes and guides the movement of the motion component Concerning the EM nanocommunication system, this would correspond to the omni/directional antenna The perturbation componen refers to the mechanism required to accommodate the message carrier to the medium in order to transmit the signal that contains the message (equivalent to modulation) Finally, specificity makes reference to the reception of the message carrier by a specific receive (receptor sensitivity/antenna aperture)

This framework is compared to the Open System Interconnection (OSI) model in order to place the five aforementioned components in the traditional communication protocol stack, as specified in Table (extracted from [10]) Due to their tiny size and their close relation to physical aspects, the nanoscale framework components are situated in the lower layers of the OSI stack, even breaching the separation between them In section 3, we will discuss this issue, analyzing the function and requirements of each component

\subsection{Metrics}

The third section of the standard addresses the definition of common metrics to give information about the interoperability among system components, together with the computation and comparison of performance in nanoscale communication network Evaluating networks by
Table 1 OSI to nanoscale communication network mapping

\begin{tabular}{|c|c|c|c|}
\hline $\begin{array}{l}\text { OSI protocol } \\
\text { layer }\end{array}$ & \multicolumn{3}{|c|}{$\begin{array}{l}\text { Framework nanoscale } \\
\text { component }\end{array}$} \\
\hline Application & \multicolumn{3}{|c|}{-} \\
\hline Presentation & \multicolumn{3}{|l|}{-} \\
\hline Session & \multicolumn{3}{|l|}{-} \\
\hline Transport & \multicolumn{3}{|l|}{-} \\
\hline Network & & Field & \\
\hline Data Link & Specificity & \multirow[b]{2}{*}{ Motion } & \\
\hline Physical & $\begin{array}{l}\text { Message } \\
\text { Carrier }\end{array}$ & & Perturbation \\
\hline
\end{tabular}

using these metrics, researchers can measure and objectively compare the grade of improvement or deterioration that different nanoscale network designs experience

The standard classifies the metrics in function of each component So, metrics related to the message carrier measure how the transmitted information is influenced by the radio channel Typical network metrics, such as message lifetime (a message carrier is discarded when exceeding a given time-to-live [TTL]) or information and communication energy (the energy required to move and steer a message carrier) are proposed for this component On the other hand, metrics referring to the motion component differ from usual network metrics and focus on the physics behind the message carrier transmission through the medium Note that these metrics mainly evaluate molecular communications Something similar occurs with the metrics related to the field component, which copes with the extent to which the message carrier motion can be controlled, evaluating whether it follows an intended gradient Specificity metrics point to the capacity of the message carrier to deliver a message to a specific destination These metrics, in fact, are quite similar to those used in conventional EM links Specificity (percentage of message carriers not addressed to an intended nanodevice which are not accepted by the intended nanodevice), sensitivity (percentage of message carriers addressed to an intended nanodevice which are checked and processed by the correct intended nanodevice), or angular spectrum (quantifying the distribution of the intensity of nanoscale communication signals received at each nanodevice as a function of angle-of-arrival), are some of the metrics suggested for this component

Finally, the standard offers some other general metrics to assess the performance of the entire nanoscale network For instance, the metric bandwidth-volume ratio, included in this segment, is employed to evaluate the total amount of information exchanged by nanodevices belonging to the nanoscale network, divided by the total system volume 
CHAPTER 2. THE IEEE 1906.1 STANDARD: NANOCOM. AS A NEW

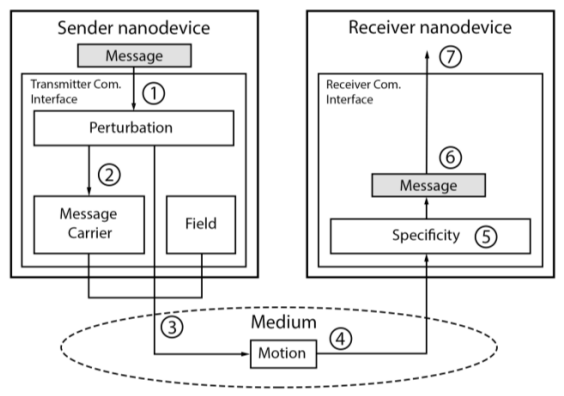

Figure 2 EM communication reference model

\subsection{EM communication reference model}

Figure 2 illustrates the general communication reference model of the standard extended to EM communications Also, the sequence of steps followed to carry out a communication between two nanodevices (in that order) is displayed They are enumerated and commented on in the following paragraphs

1) The sender nanodevice receives a message from the upper layers, in particular, a string of bits encoding the message to be dispatched This message is delivered to the Transmitter Communication Interface

2) The Perturbation component generates the message carrier, considering parameters characterizing the EM transmission, for instance, the central frequency in the $\mathrm{THz}$ band to transmit, the bandwidth (usually from $055 \mathrm{THz}$ to $155 \mathrm{THz}$ ), the transmission power, pulse features, type of modulation, etc Regarding modulation, the Time-Spread On-Off Keying (TS-OOK) modulation is the most widely extended because it is a straightforward scheme that sharply decreases the implementation complexity, alleviating the processing and computing tasks of nanodevices

3) The Transmitter Communication Interface triggers the propagation in the physical medium by passing through the Message Carrier, Perturbation, and Field components Regarding this last component, an omnidirectional antenna is employed

4) The Motion component is created in function of the propagation model in the scenario under consideration (e $g$ the human body), and takes into account requirements such as path loss or background noise [12] to modify properties of the message carrier, for instance, propagation loss or end-to-end delay

5) The receiver Specificity component checks and verifies that all the aforementioned parameters stored within the received message carrier are the same as those contained in the receiver Perturbation component

6) In the case that step 5) is correctly carried out, the message carrier is delivered to the receiver nanodevice

7) Finally, the message is dispatched to the upper layers of the receiver
In order to provide a common development environment, the standard proposes the discrete-event and open source network simulator denoted as NS-3 to integrate all the aforementioned steps and components The objective is that future investigation in the field of nanoscale communications has a starting point for exploiting all the power of the IEEE P1906 1 standard To this purpose, the simulator follows a hierarchical modular structure, dividing the EM communication implementation into two groups both taking into account the guidelines of the standard Specifically, the first group develops the five main framework components, while the second implements other secondary entities involved in the communication process but not classified as "components" (i e , communication interface, transmitter communication interface, receiver communication interface, medium, and net nanodevice) It is worth remarking that the software developed under the NS3 simulator supports the interaction of all these modules, offering a complete communication scheme

\section{IEEE P1906.1 STANDARD WEAKNESSES IDENTIFIED FOR EM COMMUNICATIONS}

Once the main features of the IEEE P1906 1 standard have been introduced, we identified several aspects which make the standard excessively open or even a not well-defined approach In this section, we discuss some of the issues not thoroughly covered by the standard

First of all, we should indicate the difficulty of giving a general definition of the concept "nanoscale communication network", since it requires the inclusion of requirements from two different scientific fields, namely Molecular and EM They are so different, that concepts such as "network" and "communication" may have different meanings in each discipline In addition, in order to maintain the generality of the definition, a communication system is considered at the nanoscale when one or more essential system components are sized at nanometers in at least one dimension Actually, following the guidelines of this definition, most works already published about EM nanocommunications [6], [8], [9], [13] (and therefore, prior to the IEEE P1906 1 standard -draft- was launched) would be included under the umbrella of the standard, since antennas employed in these studies are at the nanoscale In detail, as can be seen in Table 2 (extracted from [10]), the THz waves radiated by graphene or CNT antennas are both considered "components below $100 \mathrm{~nm}$ " and therefore "non-standard physics" So, although these studies built their designs from microscale electronic devices (and thus, the resulting design is at the microscale), the employment of $\mathrm{THz}$ waves as message carriers is enough to consider the communication at the nanoscale As can be observed, the concept of "nanoscale communication network", is diffuse enough to consider microdevices operating in a nanonetwork

Concerning the physical level, the restrictions on the amount of available energy in each nanodevice (we name them nanodevices, although their dimensions may be at the microscale) has an important impact on the communication 


\section{CHAPTER 2. THE IEEE 1906.1 STANDARD: NANOCOM. AS A NEW}

54

SOURCE OF DATA

scheme The most accepted solution for powering nanodevices involves the use of piezoelectric nanogenerators [6], [8], [13], which are able to convert mechanical strains (e g bloodstream movement) into electric energy The energy harvested is stored in a nanocapacitor to feed the nanodevice components when the energy level exceeds a given threshold Nevertheless, the main drawback to these nanogenerators is the scarce amount of energy harvested per unit of area, which strictly limits the communication capabilities of nanodevices In addition, the available energy depends on the physical medium in which nanodevices are deployed (if nanodevices take advantage of environmental movement, the energy harvested will be greater than in a static medium) and the area of the nanogenerator On the other hand, parameters related to the transmission and reception of EM waves, such as power transmission or signal to noise ratio (SNR), are not treated by the IEEE P1906 1 standard This recommendation should attract even more attention when human bodies are involved, since the high transmission power envisaged for nanodevices [9] could affect health The SNR at reception is also an important parameter to consider in order to ensure robust and reliable nanoscale communications Although the standard deals with the channel capacity (computed by using the Shannon theorem), and therefore, calculating the upper limit for the physical data rate, in the case of a low SNR value, the receiver would not be able to demodulate the radio signal

Aside from the shortcomings concerning the physical layer, we have also noticed a remarkable insufficiency of the IEEE P1906 1 standard to give some recommendations about the data link layer As can be observed in Table 1, the standard places the framework components specificity and motion at the data link layer In EM communications, these components are identified with signal radiation (motion) and antenna aperture in reception (specificity) -see Table 2However, as EM nanoscale communication networks must contain a huge number of nanodevices due to thei extremely limited transmission range (derived from the high path loss suffered in the $\mathrm{THz}$ band [12], [14]), some techniques are required to enhance the data transmission robustness between adjacent nanodevices Specifically, medium control access to arbitrate transmissions and avoid message collisions, flow control to encompass the bitrate of the communication link, or error detection mechanism would be required In addition, the number of fields and control/payload/footer length of the reference message is not defined by the standard, which could lead to the design of different and even non-interoperable data link layers

Concerning the network layer, nanodevices may have to reply to a request from an external macroscale device o may need to immediately report new events to external end personnel (e $g$ a doctor) Due to the very limited transmission range of nanodevices, this information flow could require the creation of multi-hop routes The IEEE P1906 1 standard establishes the field component as a piece/part of the network layer, but it does not cover the functionalities related to multi-hop end-to-end communications In addition, the interconnection of the
Table 2 Example of the equivalence between EM nanoscale network components and the IEEE P1906 1

\begin{tabular}{|l|l|}
\hline $\begin{array}{l}\text { IEEE P1906.1 } \\
\text { component }\end{array}$ & Implemented component \\
\hline Transmitter & CNT-based nanoantenna \\
\hline Receiver & CNT-based nanoantenna \\
\hline Message & Sodium concentration \\
\hline Medium & Air \\
\hline Message carrier & Electromagnetic (EM) wave \\
\hline Component $<100 \mathrm{~nm}$ & $\begin{array}{l}\text { Sensor, message carrier } \\
\text { (THz frequency wave) }\end{array}$ \\
\hline Non-standard physics & Impact of scale on resonance \\
\hline Motion & Radiation and waveguide \\
\hline Field & Intensity/directional antenna \\
\hline Perturbation & RF modulation \\
\hline Specificity & $\begin{array}{l}\text { Receptor sensitivity/antenna } \\
\text { aperture }\end{array}$ \\
\hline
\end{tabular}

nanoscale communication network with the macro world is an issue not considered by the standard

Higher OSI layers could be implemented, including traditional functions (e g security techniques to improve the privacy of data); however, due to extremely restricted nanodevice capabilities regarding processing, energy harvesting or memory, serious doubts have been posed about their feasibility

\section{IEEE P1906.1 STANDARD OPEN ISSUES ON EM COMMUNICATIONS}

Analyzing the shortcomings identified in the IEEE P1906 1 standard, we suggest some tips that should be considered in future EM nanoscale communications studies in order to offer the scientific community ways of confronting open research challenges not treated by the standard

As previously mentioned, one of the main goals of the IEEE P1906 1 standard is to join efforts towards the development of nanoscale communications, so the lack of a strict definition leaves the door open to different considerations The ambiguity of the definition may be a practical reason why the IEEE P1906 1 standard has not been taken into account in recent nanoscale communication works [15] [18] Therefore, we believe that a more detailed standard definition should be elaborated to better define the appropriate setting for developing future interoperable nanoscale communication networks, subject to common conditions In particular, the definition should include, firstly, the concept of a nanodevice as a device at the nanoscale, and, secondly, the division of the standard into two clearly separated parts, one focused on EM communications and the other specifically for molecular nanoscale communications The result would be a suitable definition in order to provide a more complete 
CHAPTER 2. THE IEEE 1906.1 STANDARD: NANOCOM. AS A NEW

standardization, encompassing the true dimension of communication nanonetworks

Regarding the reference energy model, more effort should be devoted to characterizing the functions of a nanoscale energy generator and its operating conditions Thus, we believe that the standard should include a reference energy model, considering the energy harvesting restrictions of nanodevices due to their tiny size (and, therefore, pointing to the available area in the nanodevice for the nanogenerator) and the environment under study So, this reference model would establish a more solid starting point to quantifying important aspects of communication, such as coverage area, size of the message to transmit, etc, which can be consistently used to develop realistic communication protocols Furthermore, from our point of view, the standardization of both maximum and recommended power transmission values would be relevant, in order to set a common power consumption model for nanodevices forming the nanonetwork If these power transmission values could be set, it would be possible to estimate the amount of energy that a nanodevice can waste (most of the required energy is dedicated to transmitting a message [6]) These values could vary depending on the application environment of the nanonetwork In addition, an SNR value recommendation should be taken into consideration by the standard, to appropriately demodulate the signal arriving to the receiver Power transmission and SNR, together with the path loss model obtained for each physical medium (e $\mathrm{g}$ human body tissues) would clearly determine the transmission range of each nanodevice for the scenario under study, which would be useful, for instance, in the planning of the required number of nanodevices deployed to cover a particular area

As regards the data link layer, some techniques are needed to improve the data transmission robustness between neighboring nanodevices We divide them into four subgroups: (i) media access, (ii) flow control, (iii) addressing, and (iv) error detection/correction Firstly, due to the very high density of nanodevices expected for nanonetwork deployment, straightforward media access control should regulate the access to the radio channel, to manage simultaneous transmissions in the transmission medium For instance, by using random seeds to activate th nanodevice transceiver and listen to the medium, message collisions will be mitigated In the case that a medium access control technique is not employed, messages dispatched by neighbors could collide, corrupting a high percentage of the transmitted data Secondly, for the same reason, a flow control mechanism is essential to coordinate the communication between nanodevices For example, simple acknowledgement reply to confirm the reception of a message, together with a waiting timer for retransmissions (when collisions occur) could be enough to control the traffic load in the network Thirdly, every single nanodevice in the network requires a unique ID to be identified, facilitating the transmissions from a source nanodevice to a remote destination Finally, error detection methods are mandatory to evaluate the standard metrics, such as sensitivity or specificity, since false positives must be
Table 3 Weaknesses and open issues for the IEEE P1906 1 standard for EM communications

\begin{tabular}{|l|l|}
\hline $\begin{array}{l}\text { Standard weaknesses } \\
\text { for EM } \\
\text { communications }\end{array}$ & Open issues \\
\hline $\begin{array}{l}\text { Excessively open } \\
\text { definition of nanoscale } \\
\text { communication network }\end{array}$ & $\begin{array}{l}\text { More detailed definition of } \\
\text { nanoscale EM communication } \\
\text { devices Two separate chapters } \\
\text { for molecular and EM } \\
\text { nanocommunication are } \\
\text { suggested }\end{array}$ \\
\hline $\begin{array}{l}\text { Lack of a reference } \\
\text { energy model }\end{array}$ & $\begin{array}{l}\text { Definition of a general enough } \\
\text { energy model, but easily } \\
\text { adapted to the technology } \\
\text { employed Energy restrictions } \\
\text { should be better quantified to } \\
\text { design a nanonetwork offering } \\
\text { a real service }\end{array}$ \\
\hline $\begin{array}{l}\text { SNR is not contemplated } \\
\text { in the reference } \\
\text { communication model }\end{array}$ & $\begin{array}{l}\text { Standardization of SNR values } \\
\text { expected at reception to } \\
\text { calculate appropriate receiver } \\
\text { sensitivity thresholds }\end{array}$ \\
\hline $\begin{array}{l}\text { Lack of layer 2 } \\
\text { techniques to enhance } \\
\text { communication } \\
\text { robustness }\end{array}$ & $\begin{array}{l}\text { Recommendation of } \\
\text { techniques referring to media } \\
\text { access control, addressing } \\
\text { scheme, flow control and error } \\
\text { detection }\end{array}$ \\
\hline $\begin{array}{l}\text { Equivalent layer } 3 \text { OSI } \\
\text { reference model } \\
\text { functions are not } \\
\text { rigorously addressed }\end{array}$ & $\begin{array}{l}\text { Definition of routing } \\
\text { procedures to allow multi-hop } \\
\text { end-to-end communications } \\
\text { Design of a complete network } \\
\text { topology }\end{array}$ \\
\hline $\begin{array}{l}\text { Interconnections between } \\
\text { a nanoscale } \\
\text { communication network } \\
\text { and macroscale devices } \\
\text { are not addressed }\end{array}$ & $\begin{array}{l}\text { Design and development of a } \\
\text { link between the nano and } \\
\text { macroscale worlds }\end{array}$ \\
\hline
\end{tabular}

properly detected Hence, we believe the standard should include these data link layer aspects, to provide a more robust and reliable nanoscale communication framework

The interconnection of nanodevices and their respective links with existing communication networks entails the design of network architectures that have to be properly planned So far, we think that the tree-based topology is the most appropriate for EM nanonetworks, which should be further divided into different hierarchical layers [19] The lowest level is composed of nanodevices grouped into clusters Each cluster is connected to a larger and more powerful device (in terms of processing, memory, and energy consumption), which belongs to the upper level (e g a nano-router) Finally, in the top layer is the gateway which interconnects the nanonetwork with the macro world With this topology, a straightforward multi-hop routing algorithm should be designed to convey the data collected by the nanodevices in the lowest level, ultimately, to the Internet 


\section{CHAPTER 2. THE IEEE 1906.1 STANDARD: NANOCOM. AS A NEW}

Table 3 summarizes the identified limitations of current standard and associates them with their corresponding open issues Note that even a simple solution to these open issues is a real research challenge at the nanoscale

\section{CONCLUSIONS}

The IEEE P1906 1 standard establishes a set of recommended practices with the aim of allowing researchers to advance in the development of effective nanoscale communication systems Even though it supposes a sound step forward, more concreteness is necessary to envisage a common framework which can become a solid foundation for designing forthcoming EM nanonetworks Keeping this premise in mind, we have first reviewed the main body of the standard, highlighting those definitions, metrics, and components related to EM communications In addition, we have taken advantage of the general communication model proposed by the standard, and contributed with a refined reference communication mode adapted to EM communications Secondly, we have identified some relevant shortcomings of the standard, dividing them into four main groups The first discusses the generality of the definition of the term "nanoscale communication network" itself, while the three remaining groups reveal important deficiencies in each of the three lowest layers of the OSI reference model (physical, data link and network layers) Finally, we have offered possible guidelines for addressing each detected weakness in order to enhance the feasibility and capabilities of EM nanoscale communications

\section{REFERENCES}

[1] I Llatser, C Kremers, A Cabellos-Aparicio, J M Jornet, E Alarcón, and D N Chigrin, "Graphene-based nano-patch antenna for terahertz radiation," Photonics Nanostructures - Fundam. Appl., vol 10, no 4, pp 353 358 , Oct 2012

[2] J M Jornet and I F Akyildiz, "Graphene-based plasmonic nano-transceiver for terahertz ban communication," in The 8th European Conference on Antennas and Propagation (EuCAP 2014), 2014, pp 492-496

[3] M M Shulaker et al., "Carbon nanotube computer," Nature, vol 501, no 7468, pp 526-530, Sep 2013

[4] M Moore et al., "A Design of a Molecular Communication System for Nanomachines Using Molecular Motors," in Fourth Annual IEEE International Conference on Pervasive Computing and Communications Workshops (PERCOMW'06), pp 554

[5] I F Akyildiz, F Brunetti, and C Blázquez, "Nanonetworks: A new communication paradigm," Comput. Networks, vol 52, no 12, pp 2260-2279, Aug 2008

[6] S Canovas-Carrasco, A -J Garcia-Sanchez, F GarciaSanchez, and J Garcia-Haro, "Conceptual Design of a Nano-Networking Device," Sensors, vol 16, no 12, p 2104,2016
[7] J M Jornet, J Capdevila Pujol, and J Solé Pareta, "PHLAME: A Physical Layer Aware MAC protocol for "PHLAME: A Physical Layer Aware MAC protocol for
Electromagnetic nanonetworks in the Terahertz Band," Nano Commun. Netw., vol 3, no 1, pp 74-81, 2012

[8] J M Jornet and I F Akyildiz, "Joint Energy Harvesting and Communication Analysis for Perpetual Wireless Nanosensor Networks in the Terahertz Band," IEEE Trans. Nanotechnol., vol 11, no 3, pp 570-580, May 2012

[9] J M Jornet and I F Akyildiz, "Femtosecond-Long Pulse-Based Modulation for Terahertz Band Communication in Nanonetworks," IEEE Trans. Communication in Nanonetworks," IEEE Tran
Commun., vol 62, no 5, pp 1742-1754, May 2014

[10] IEEE Recommended Practice for Nanoscale and Molecular Communication Framework IEEE Std $19061-2015,2015$

[11] Nanotechnologies-Terminology and Definitions for Nano-Objects-Nanoparticle, Nanofibre and Nanoplate ISO/TS 27687:2008, 2008

[12] K Yang, A Pellegrini, M O Munoz, A Brizzi, A Alomainy, and Y Hao, "Numerical analysis and characterization of $\mathrm{THz}$ propagation channel for bodycentric nano-communications," IEEE Trans. Terahertz Sci. Technol., vol 5, no 3, pp 419-426, 2015

[13] I F Akyildiz and J M Jornet, "Electromagnetic wireless nanosensor networks," Nano Commun. Netw., vol 1, no 1, pp 3-19, 2010

[14] J M Jornet and I F Akyildiz "Channel Capacity of Electromagnetic Nanonetworks in the Terahertz Band," Commun. (ICC), 2010 IEEE Int. Conf., pp 1-6, 2010

[15] Y Jian et al., "nanoNS3: A network simulator for bacterial nanonetworks based on molecular communication," Nano Commun. Netw., vol 12, pp 111,2017

[16] S E Hosseininejad et al., "Study of hybrid and pure plasmonic terahertz antennas based on graphene guidedwave structures," Nano Commun. Netw., vol 12, pp 34 42, 2017

[17] M A Zainuddin, E Dedu, and J Bourgeois, "Lowweight code comparison for electromagnetic wireles nanocommunication," IEEE Internet Things J., vol 3 , no 1 , pp 38-48, 2016

[18] A Tsioliaridou, C Liaskos, E Dedu, and S Ioannidis, "Packet routing in 3D nanonetworks: A lightweight, linear-path scheme," Nano Commun. Netw., vol 12, pp 63-71, 2016

[19] I Akyildiz and J Jornet, "The Internet of nano-things," IEEE Wirel. Commun., vol 17, no 6, pp 58-63, Dec 2010 
Chapter 3

A nanoscale communication
network scheme and energy
model for a human hand scenario 
CHAPTER 3. A NANO COM. NETWORK SCHEME AND ENERGY

Nano Communication Networks 15 (2018) 17-27

\begin{tabular}{|c|c|c|}
\hline gentis & $\begin{array}{l}\text { Contents lists available at ScienceDirect } \\
\text { Nano Communication Networks }\end{array}$ & $\begin{array}{l}\text { Nano } \\
\text { Communication } \\
\text { Neteritas }\end{array}$ \\
\hline ELSEVIER & journal homepage: www.elsevier.com/locate/nanocomnet & \\
\hline
\end{tabular}

\section{A nanoscale communication network scheme and energy model for a} human hand scenario

Sebastian Canovas-Carrasco, Antonio-Javier Garcia-Sanchez* , Joan Garcia-Haro

Department of Information and Communication Technologies, Universidad Politécnica de Cartagena (UPCT) Campus Muralla del Mar, Department of Information
Cartagena E-30202, Spain

\section{A R T I C L E I N F O}

Artide history:

Received 20 July 2017

Received in revised form 27 December 2017

Accepted 22 January 2018

Available online 31 January 2018

Keywards:

Wireless nanosensor networks

Terahertz band

Energy harves

Human-body scenario A B S T R A C T
Real-time monitoring of medical test parameters as well as biological and chemical substances inside
the human body is an aspiration which might facilitate the control of pathologies and would ensure
better effectiveness in diagnostics and treatments. Future Body Area NanoNetworks (BANN) represent an
ongoing effort to complement these initiatives, although due to its early stage of development, further
research is required. This paper contributes with a hierarchical BANN architecture consisting of two
types of nanodevices, namely. nanonodes and a nanorouter. which are conceptually designed using
technologically available electronic components. A straightforward communication scheme operating at
the THz band for the exchange of information among nanodevices is also proposed. Communications are
conducted in a human hand scenario since, unlike other parts of the human body. the negative impact
of path loss and molecular absorption noise on the propagation of electromagnetic waves in biological
tissues is mitigated. However, data transmission is restricted by the tiny size of nanodevices and their
extremely limited energy storing capability. To overcome this concern, nanodevices must be powered
through the bloodstream and external ultrasound energy harvesting sources. Under these conditions, the
necessary energy and its management have been thoroughly examined and assessed. The results obtained
reveal the outstanding ability of nanonodes to recharge, thus enabling each pair of nanonode-nanorouter
to communicate every 52 min. This apparently long period is compensated by the considerably high
number of nanonodes in the network, which satisfies a quasi-constant monitoring of medical parameter
readings.
02018 The Authors. Published by Elsevier B.V. This is an open access article under the CC BY-NC-ND

O 2018 The Authors. Published by Elsevier B.V. This is an open access article under the CC BY-NC-ND

\section{Introduction}

Nanonetworks will be a powerful technological solution enabling the detection, acquisition and monitoring of physical magnitudes in application scenarios which are hitherto unimaginable. It is even possible to foresee data processing, the communication of results, and decision-making tasks, all being carried out on a nanoscale level and with unprecedented accuracy. Medical parameters such as body temperature, glucose levels or cancer biomarker eters such as body temperature, glucose levels or cancer biomarker destinations through nanodevices (in this work, for nanodevices we mean nanomachines based on electronics with a scale measured in nanometers), which will play an important role in the field of nanonetworking. In this regard, one of the most promising applications of nanonetworks is their use as Body Area NanoNetworks (BANN); in which, for instance, interconnected nanodevices flow into the bloodstream, reporting information to specialist external

\footnotetext{
* Corresponding author.

E-mail addresses: sebas canovas甲upct.es (S. Canovas-Carrasco). antoniojavier.garciađupct,es (A-J. Garcia-Sanchez). joang.haroФupct.e (J.-Garcia-Haro).
}

personnel (e.g. doctors) or information processing systems (e.g. Big Data paradigm)

However, the design, implementation and deployment of nanonetworks in a living biological environment poses considerable problems, which must be overcome; in turn presenting challenges in many different research fields such as signal propagation, antennas, and information technology, among others. The extremely high electromagnetic (EM) frequencies required for communication among nanodevices (expected in the $\mathrm{THz}$ band) togetherwith the particular channel requirements in biological tissues (extremely high path loss and molecular absorption noisevalsues (extremely high path loss and molecular absorption noise val-
ues) restrict signal propagation. A possible approach consists of increasing transmission power in order to successfully send/receive data. However, due to the tiny size of nanodevices, their available energy is highly limited. Under these circumstances, a trade-off between both concerns (transmission power and consumed energy) must be analyzed and quantified. Unfortunately, according ergy) to the best of our knowledge, few studies in literature tackle this
problem $[1,2]$.

Therefore, more effort must be devoted to developing a realistic energy model that would guarantee sufficient power to appropriately supply the nanodevices. In this paper, firstly, we contribute 


\section{CHAPTER 3. A NANO COM. NETWORK SCHEME AND ENERGY MODEL FOR A HUMAN HAND SCENARIO}

with the design and development of a specific energy model for a BANN, which considers and evaluates the concerns related to the channel and the nanoscale. To minimize the negative impact of signal propagation and molecular absorption losses, we consider a human hand scenario, given the thinness of its biological tissues. Unlike other BANN works, a detailed study of radio propagation losses is carried out, taking into account the most relevant biological tissues of the human hand scenario under study.

To achieve energy consumption attuned to the needs of mission-driven nanodevices, a straightforward communication scheme in a thorough design of a network architecture is proposed and assessed. Both the network architecture and communication scheme constitute the second contribution of this work, discussing the facilities for sensing a biological parameter and transmitting it outside the human body. To this end, we propose a hierarchical architecture composed of two types of nanodevices, which we also design conceptually. Namely, (i) nanonodes circulating in (ii) nanorouter implanted into the ski nanorouter, and outside the body, a gateway connects the BANN to the external world, via conventional technologies, such as Bluetooth or WiFi. It is worth mentioning that all the components of the nanodevices have been selected from advanced but available technologies.

Finally, our third contribution relates to energy management, in particular, to supplying the appropriate power to nanodevices to enable them to conduct their tasks. Due to the unfeasibility of to enable them to conduct their tasks. Due to the unfeasibility of ies, the power supply system in both nanonode and nanorouter, should enable energy harvesting and storage. In this endeavor, piezoelectric nanowires and a rechargeable nanocapacitor have to be integrated into the nanodevices. Considering the scenario and the architecture here proposed, the power provision for nanonodes is achieved by employing two types of energy harvesting sources: (i) the bloodstream of the human body itself, and (ii) an ultrasound external source placed in the hand. Both induce movements in the nanowires, ensuring a constant battery recharge. Conversely, the nanorouter battery is continuously recharged by the ultrasound external power supply.

The rest of the paper is organized as follows. In Section 2, we review the related work in the area. In Section 3, we propose a layered channel model that simulates the dorsum of a human hand. Concerning the biological channel, we analyze both path loss and molecular absorption noise. Section 4 is devoted to determining the power consumption of a nanonode and a nanorouter. Likewise, in Section 5, the energy harvesting rate is accurately calculated to obtain the time required by a nanonode to recharge its nanocapacitor. Finally, in Section 6 , a communication scheme is offered, subject to the particular restrictions of the scenario under consideration. Section 7 concludes this work.

\section{Related work}

To the best of our knowledge, a realistic case of study for a BANN contributing with an exhaustive work in terms of nanodevice design, integrating communication mechanisms to extend the lifetime of nanodevices has not been reported yet in the scientific literature. In this regard, different papers have tackled specific asliterature. In this regard, different papers have tackled specific as-
pects referring to nanocommunications, such as network topology, pects referring to nanocommunications, such as network topology, communication schemes, channel characterization, or the energy consumption of nanodevices. However, unlike our work, these nanocommunication topics are regarded as isolated islands, and a comprehensive and $\mathrm{m}$

of them is not offered.

Concerning BANN topologies, diverse studies have dealt with how a BANN should be deployed $[3,4]$, proposing a generic hierarchical architecture consisting of a nanointerface receiving external requests and returning the requested data, a nanorouter collecting data from nanonodes, and in turn, the nanonodes gathering data from the human body. However, the communication scheme portrayed did not suggest any solution to overcoming the huge absorption losses in the biological tissues at the $\mathrm{THz}$ band. Thus, the energy required to accomplish the communication between nanodevices must be significantly higher than the value employed in the communication schemes described in these works. As result, the packet exchange suggested between nanorouter and nanonode cannot be feasibly implemented.

Continuing with works related to the communication scheme in a nanonetwork, in [5], a handshake-based MAC protocol for nanonetworks is proposed. In this protocol, nanodevices are able to dynamically choose different physical layer parameters based on the channel conditions and their remaining energy. Nevertheless, there are two main drawbacks not covered by this protocol. Firstly contemplating a realistic nanonode design [6], the handshake process requires an amount of energy in the nanonode that would deplete the energy stored in its nanocapacitor without sending any useful data. Secondly, nanonodes might not have enough computational resources to find and share the optimal channel communication parameters. In [7], authors designed a routing framework for nanonetworks to optimize energy consumption, thus developing a multi-hop decision algorithm. In this approach, the nanorouter decides upon the distance to the nanonodes; if they must send the data directly to the nanorouter or through multi-hop transmission. However, due to the extremely limited capability of nanodevices in terms of processing and memory, they should employ a simpler communication scheme. It should be mentioned that these studies are mainly focused on general Wireless NanoSensor Networks (WNSN), while the design of energy-efficient protocols for BANNs, remains scarcely explored. In this paper, we provide a straightforward communication scheme adapted to a realistic scenario in order to accomplish communications, considering the limited resources of nanodevices.

Regarding the $\mathrm{THz}$ channel characterization, in [8], a propagation model for EM communications in the THz band is developed. The radiative transfer theory and molecular absorption are considered in this work. The model accounts for the total path loss and the molecular absorption noise that a wave in the $\mathrm{THz}$ band experiences when propagating over very short distances. In particular, for biological tissues, the characteristics of EM waves propagating inside the human body at terahertz frequencies was studied in [1]. Based on the obtained path losses and noise level in different human tissues (skin, fat, and blood), authors evaluated the channel capacity within the human body. However, the total path loss was not computed in a realistic scenario. In this context, the work in [9], took into account the inhomogeneous structure of biological tissues to calculate path loss in a biological medium. Even though the path loss obtained was extremely accurate, a communication scheme to accomplish communication between nanodevices was not contemplated. To face these challenges, our paper considers the path loss in a human hand to properly design a communication scheme for a BANN.

With regards to energy management in a nanonetwork, the pioneering work of Jornet et al. [2], proposed a theoretical analysis of energy harvesting in a nanonetwork. This model was designed considering both the energy harvesting and the energy consumption processes. As in our paper, authors contemplated a capacitor to store energy collected from a piezoelectric nanogenerator. However, authors did not consider current technologies to estimate the maximum energy stored in a nanonode, nor higher transmitted energy values to overcome the channel loss of biological tissues in the $\mathrm{THz}$ band. 


\section{CHAPTER 3. A NANO COM. NETWORK SCHEME AND ENERGY}

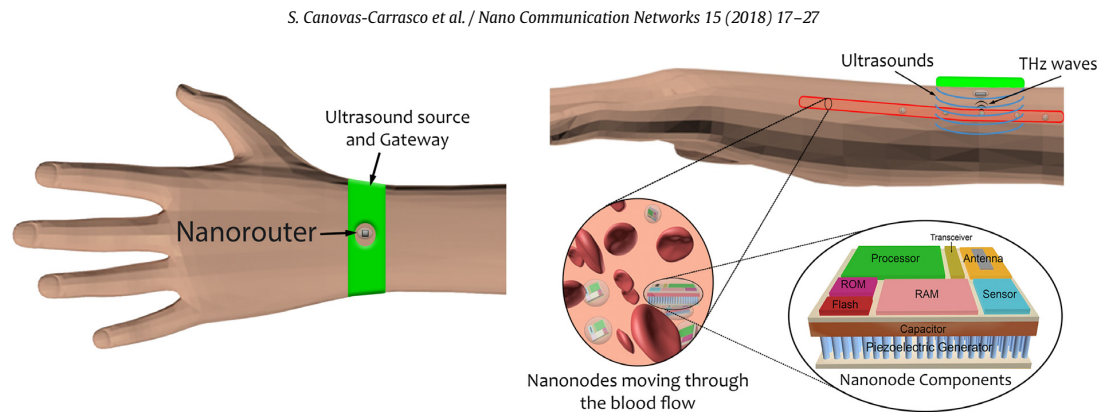

Fig. 1. Hierarchical BANN architecture.

\section{Terahertz biological channel model}

In this section, the characterization of a biological channel in the $\mathrm{THz}$ band is proposed. The dorsum of a hand is taken as a reference scenario, which is modeled as a 4-layered structure (each layer corresponds to a specific type of biological tissue). Once the channel composition is defined, we compute the total path loss by chang the main electrical properties of analyzing the main electrical properties of each type of biological tissue at $1 \mathrm{THz}$. Finally, the molecular noise is also studied to determine its incidence on the communication scheme.

\subsection{Scenario under study/channel composition}

The scenario under study is illustrated in Fig. 1 where an external portable device (gateway), placed at the dorsum of a hand, feeds the nanorouter by ultrasounds. The nanorouter is implanted into the skin to be able to communicate with the nanonodes, which are in constant movement in the bloodstream. Thus, to estimate the path loss between the nanonode and nanorouter, the biological composition of the dorsum of the hand has been modeled as a layered structure, similar to the model proposed in previous works $[1,9]$. The upper layer is composed of skin, split into dermis and epidermis. Both human tissues have very similar electrical properties, as demonstrated in [10], thus, in our study, we have considered both as one single tissue. The middle layer represents the subcutaneous fat, which in the back of the hand is rather thin. This thinness makes this part of the human body one of the most suitable for establishing a BANN, since the distance between the outside of the human body and the vein is fairly short (Table 1). Finally, the lower layer, which models the vein, is made up of blood. This will recreate the situation in which the nanonode is operating in the circulatory system. Both nanodevices must be encapsulated in a biocompatible capsule/artificial cell to be implanted into the human body, as cited in [11].

The thickness of each tissue, specified in Table 1, has been obtained from experimental measurements reported in scientific literature for the dorsum of the hand [12-15]. When a range of thickness is suggested in the literature, the worst case scenario, that is, the greatest thickness, has been adopted. Finally, note that that is, the greatest thickness, has been adopted. Finally, note that the blood
vein.

In our scenario, both nanorouter and nanonode are supposed to be placed inside the human body. The nanorouter is located in the skin, since its thickness allows a straightforward implantation. In our model, it is in the interface between the dermis and epidermis, facing 政 vein and thus facilitating communication with the nanonodes. The nanonodes flow through a vein, and to study the more common scenario, we consider in our model that the nanonode is positioned in the center of the vein.
Table 1

Thickness of each biological tissue (dorsum of the hand).

\begin{tabular}{ll}
\hline Biological tissue & Thickness $(\mathrm{mm})$ \\
\hline Epidermis & 0.19 \\
Dermis & 1.06 \\
Subcutaneous fat & 0.06 \\
Blood & 1.20 \\
\hline
\end{tabular}

Blood

3.2. Path loss

The model proposed by Jornet and Akyildiz in [8] to calculate the path loss of the $\mathrm{THz}$ channel in water vapor paved the way fo later path loss studies in biological tissues. Based on this approach, in [1], a theoretical path loss model is proposed, in which the channel losses are formulated as the addition of two contributions: the spreading loss and the molecular absorption loss. The first term represents the transmission of the EM wave in the physical medium, which can be easily modeled by the Friis path loss formula. We adapt this equation to our biological case of study, which is defined as:

$L_{\text {spread }}(f, d)=\left(\frac{4 \pi d n_{m}}{\lambda_{0}}\right)^{2}$

where $d$ is the propagation distance, $\lambda_{0}$ is the free-space wavelength, and $n_{m}$ stands for the refractive index of the medium. At a frequency of $1 \mathrm{THz}\left(\lambda_{0}=300 \mu \mathrm{m}\right)$, the spreading loss is considerably high, severely limiting the transmission range of nanonodes. This is the reason why our human body scenario sharply reduces the communication distance between the nanorouter and the nanonode.

The second term of the path loss accounts for the attenuation caused by molecular absorption. In general, when EM waves propagate through a material, a fraction of the EM energy radiated by a nanoantenna is converted into the internal kinetic energy of the molecules. This process depends on the working frequency and the biological tissue under consideration (i.e. it differs from a wave propagating in the skin or in the blood). Therefore, from a mathematical viewpoint, the molecular absorption of each material can be characterized by the so-called absorption coefficient $(\alpha)$, which varies with the frequency. Thus, given $\alpha(f)$, the molecular absorption path loss can be obtained by the following expression [8]:

$L_{a b s}(f, d)=e^{\alpha(f) d}$

where $d$ is the propagation distance. In order to calculate the absorption path loss at $1 \mathrm{THz}$, Table 2 shows the absorption coefficient together with $n_{m}$ for each tissue in the model at the required frequency [10]. 


\section{CHAPTER 3. A NANO COM. NETWORK SCHEME AND ENERGY MODEL FOR A HUMAN HAND SCENARIO}

Table 2 Physical properties for skin, subcutaneous fat, and blood respectively.

\begin{tabular}{lllll}
\hline Biological tissue & $\begin{array}{l}\text { Absorption coefficient at 1 } \mathrm{THz} \\
\left(\mathrm{cm}^{1}\right)\end{array}$ & Refractive index $n_{m}$ & $\begin{array}{l}\text { Acoustic absorption coefficient } \\
\left(\mathrm{dB} \mathrm{cm}^{1} \mathrm{MHz}{ }^{1}\right)\end{array}$ & $\begin{array}{l}\text { Acoustic impedance } \\
\text { (MRayl) }\end{array}$ \\
\hline Skin & 110 & 1.73 & 1.84 & 1.99 \\
Subcutaneous fat & 80 & 1.50 & 0.6 & 1.38 \\
Blood & 1.96 & 0.15 & 1.66 \\
\hline
\end{tabular}

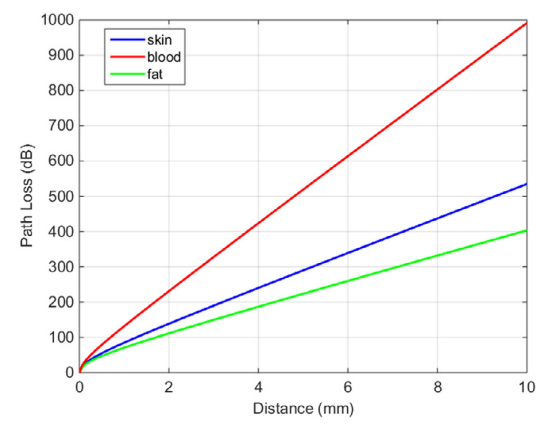

Fig. 2. Total path loss for skin, fat and blood as a function of distance at $1 \mathrm{THz}$.

Once each analytical term of the losses has been detailed, the total path loss of the channel can be expressed as follows:

$L_{\text {path }}(f, d)=L_{\text {spread }}(f, d) * L_{a b s}(f, d)=\left(\frac{4 \pi d n_{m}}{\lambda_{0}}\right)^{2} e^{\alpha(f) d}$

Analyzing the expression (3), it can be seen that the total path loss increases as the frequency, distance or absorption of the medium (or a set of parameters) rises. To be precise in our study, the total path loss must be derived for each biological tissue, since each one will impact differently on the path loss calculation due each one will impact differently on the path loss calculation due
to its specific refractive index and absorption coefficient. Therefore, to calculate $L_{\text {path }}(f, d)$ these two parameters at $1 \mathrm{THz}$ and the distance of each tissue must be taken into consideration. The dependence of the total path loss for skin, fat, and blood on the distance are shown in Fig. 2, where the huge losses per length unit can be perceived. It is noteworthy that, in accordance with the work in [1], the losses caused by reflections in the interfaces between layers are not considered due to the similar electrical properties of the tissues.
In our base scenario, the nanonode could start the information exchange with the nanorouter when it travels through the dorsum of the hand. In the same way as the nanorouter, nanonodes can harvest energy from ultrasounds if they are close enough to the gateway. Under these conditions, they send data during the period of time from the entrance into the ultrasound region to the arrival to the shortest distance point with the router (SDP), which is the location where the distance between both devices is minimal. The length of the ultrasound region, in which energy from ultrasounds can be harvested efficiently, has been considered $5 \mathrm{~mm}$ as a design parameter. This length is enough to ensure the straightforward integration of the ultrasound energy source into a small external wearable (e.g. a bracelet, as shown in Fig. 1). Thus, we calculate the total path loss in this range of distances, in accordance with the scheme depicted in Fig. 3.

Here, the distance between transmitter and receiver depends on the distance from the nanonode to the $\operatorname{SDP}(\Delta d)$. As can be seen, the fraction of distance traveled by the EM wave through the skin $\left(d_{\text {skin }}\right)$, fat $\left(d_{\text {fat }}\right)$, and blood $\left(d_{\text {blood }}\right)$ also varies, which will directly affect the total path loss. Applying basic trigonometry, $d_{\text {skin }}, d_{\text {fat }}$, and $d_{\text {blood }}$ as a function of $\Delta d$ have been correspondingly calculated:

$d_{\text {skin }}(\Delta d)=\sqrt{t_{\text {skin }}^{2}+\left(\Delta d \frac{t_{\text {skin }}}{d_{\text {min }}}\right)^{2}}$

$d_{f a t}(\Delta d)=\sqrt{\left(t_{\text {skin }}+t_{f a t}\right)^{2}+\left(\Delta d \frac{t_{\text {skin }}+t_{\text {fat }}}{d_{\min }}\right)^{2}}-d_{\text {skin }}$

$d_{\text {blood }}(\Delta d)=\sqrt{d_{\min }^{2}+\Delta d^{2}}-\left(d_{\text {fat }}+d_{\text {skin }}\right)$

where $t_{\text {skin }}, t_{\text {fat }}$, and $t_{\text {blood }}$ stand for the thickness of skin, fat, and blood, respectively, and $d_{\min }$ is the minimum distance between nanorouter and nanonode.

Under these conditions, substituting the specific values for $n_{m}$ $\alpha(f)$, and $d$ for each biological tissue in the expression (3), the total path loss as the nanonode moves across the ultrasound region is shown in Fig. 4. It can be observed that the total path loss sharply decreases as the nanonode goes into the ultrasound region (getting closer to the nanorouter) and reaches its minimum value (124 dB)

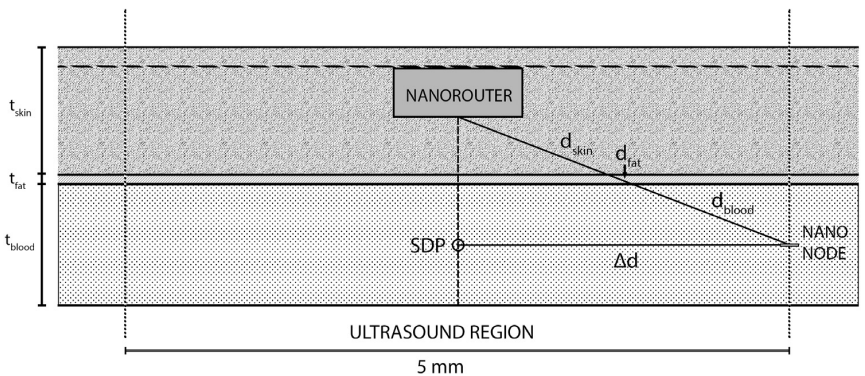

Fig. 3. Distance scheme at the ultrasound region. 


\section{CHAPTER 3. A NANO COM. NETWORK SCHEME AND ENERGY}

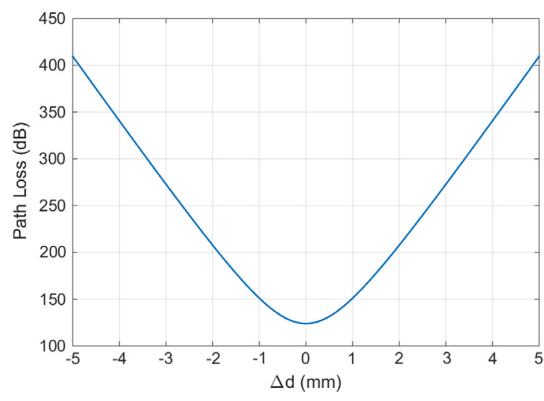

Fig. 4. Path loss as a function of the distance to the SDP.

when $\Delta d$ is equal to zero, that is, when the nanonode is in the SDP.

However, as the nanonode moves away from the SDP, the path loss quickly increases. Therefore, in order to maximize the probability of completing the communication without error (corrupted bility of completing the communication without error (corrupted
or lost information), the nanonode should be activated as close as possible to the SDP.

\subsection{Molecular absorption noise}

The absorption phenomenon from molecules present in the medium, not only affects the properties of the channel in terms of attenuation, but it also introduces noise. As was studied in depth in [8], this is the major noise contribution at the THz band. depth in [8], this is the major noise contribution at the THz band.
The intrinsic noise caused by the electronics systems based on The intrinsic noise caused by the electronics systems based on
graphene (material proposed to build nanoantennas in most researches) can be considered negligible [16,17]. To reduce the effect of molecular absorption noise, the technique proposed by Akyildiz and Jornet, based on the transmission of femtopulses, along with an On-Off Keying modulation (TS-OOK) [18], is the communication procedure chosen for the communication between nanonode and nanorouter. It employs ultra-short pulses ( $100 \mathrm{fs}$ ) to send a logical ' 1 ', while the logical ' 0 ' is transmitted as silence.

As detailed in [8], molecular noise relies on the emissivity of the channel, $(\varepsilon)$, which can be defined as a function of the absorption coefficient $(\alpha(f))$ :

$\varepsilon(f, d)=1-e^{-\alpha(f) d}$

where $d$ is the propagation distance. The equivalent noise temperature due to molecular absorption $\left(T_{m o l}\right)$ expressed in Kelvin is defined as:

$T_{\text {mol }}(f, d)=T_{0} \varepsilon(f, d)=T_{0}\left(1-e^{-\alpha(f) d}\right)$

where $T_{0}$ denotes the reference temperature, which in our case corresponds to normal human body temperature ( $310 \mathrm{~K}$ ). From (8), the noise power spectral density $(N)$ can be expressed as follows:

$N(f, d)=K_{B} T_{m o l}=K_{B} T_{0}\left(1-e^{-\alpha(f) d}\right)$

where $K_{B}$ stands for the Boltzmann constant. Thus, the noise power at the receiver $\left(P_{N}\right)$ can be calculated by integrating $N(f, d)$ over the transmission bandwidth (B):

$P_{N}(f, d)=\int_{B} N(f, d) d f=K_{B} \int_{B} T_{0}\left(1-e^{-\alpha(f) d}\right) d f$

This expression computes the noise power at the receiver. However, this noise is only present when a femtopulse is propagating through the medium, that is, when a logical ' 1 ' is being transmitted. Otherwise, the noise at the receiver is nearly zero. As the time between pulses is seven orders of magnitude larger than the pulse duration, the molecular noise power should not be detected in a different pulse period, thus reducing the effect of noise in bit detection errors. As a consequence of molecular noise behavior, the noise power is added to the power transmitted, resulting in the deforming and spreading of the pulse in time (see Fig. 5). This spread is due to the relaxation time of the molecules, and it is defined as the amount of time required for excited molecules to reduce their amplitudes below 10\% [19]. For this type of femtopulse, the relaxation time is between 1 and $5 \mathrm{ps}$, which is much shorter than relaxation time is between 1 and $5 \mathrm{ps}$, which is much shorter than
the time between pulses ( $2 \mu \mathrm{s}$, justified in Section 4 ) and therefore, intersymbol interference (ISI) is unfeasible.

Hence, the molecular noise at the receiver when a logical ' 0 ' is transmitted (the transceiver remains silent), is negligible. As the electronic noise envisaged for graphene-based devices is extremely low, the difference of energy levels between the reception of a logical ' 1 ' and a logical ' 0 ' is substantial. This implies that the proposed TS-OOK modulation is robust enough to carry out the communication between nanodevices while keeping the communication process as simple and reduced in computer complexity as possible.

\section{Nanonetworking devices: definition, features and power} consumption

As previously mentioned, the limited nanodevice energy resources lead to the use of a hierarchical nanonetwork architecture to enable the junction between macro and nano worlds, following the scheme described in [20]. As shown in Fig. 1, nanonodes are the smallest nanodevices (with just a few $\mu \mathrm{m}$ in size) in our scenario and belong to the lowest level of the hierarchy, traveling through the veins. In the upper level, the nanorouter gathers the data collected by nanonodes and is able to communicate with data collected by nanonodes and is able to communicate with
the gateway using a $\mathrm{THz}$ communication system. The gateway dispatches sensor data to a traditional external system via WiF or Bluetooth (the communication between the gateway and the nanorouter is not covered in this work). It is noteworthy that critical aspect of properly designing the communication scheme is to determine the consumption of each nanodevice. Thus, in this section, we estimate their energy consumption based on feasible section, we estimate their energy consumption based on feasible
technologies in order to design a communication schedule able to work under realistic conditions.

\subsection{Nanonode power consumption}

The energy required by a nanonode to communicate with a nanorouter should be accurately determined to accomplish specific application, since the operating environment conditions change according to the scenario under study (varying the amount of harvested energy). To address this problem, we refer to a previous work [6], in which the nanodevice proposed is in the lowest level of the nanonetwork hierarchy (nanonode). A nanonode must gather, store and process sensor information to later transmit it via a $\mathrm{THz}$ band communication system. These tasks should be carried out under an appropriate energy consumption scheme resulting in an acceptable recharge time for nanodevices. In this proposal, the nanodevice is divided into five main components (see Fig. 1) namely: (i) nanoprocessor; (ii) memory nanomodules (RAM and ROM memories), (iii) graphene radiocommunication nanosystem, (iv) nanosensor, and (v) energy nanogenerator. In order to encompass all these components in a tiny device, the dimensions of the nanonode proposed in [6] are $8 \times 8 \times 3 \mu \mathrm{m}$. In the following subsections, the first four components are reviewed, paying special attention to power consumption, while the energy required for the nanogenerator is further analyzed in Section 5. 


\section{CHAPTER 3. A NANO COM. NETWORK SCHEME AND ENERGY MODEL FOR A HUMAN HAND SCENARIO}

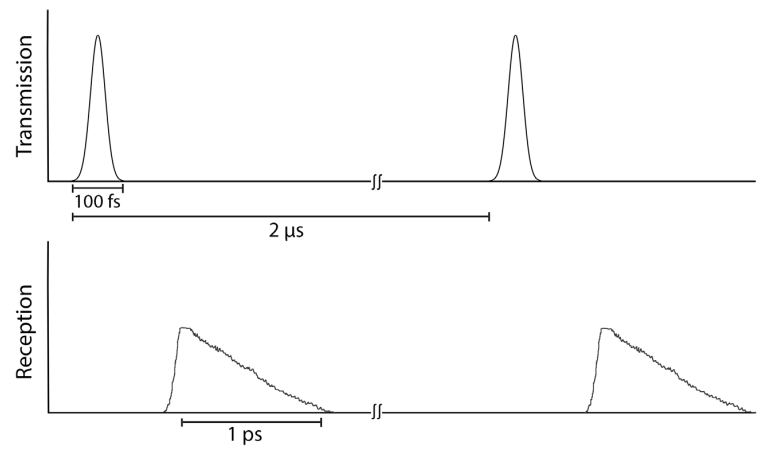

Fig. 5. Pulse spreading at the receiver due to molecular noise.

4.1.1. Nanoprocessor

The nanoprocessor is responsible for handling the basic protocol instructions received from the nanorouter, such as waking up/sleeping, reading data from the sensor or transmitting a frame, among others. Moreover, it will drive the operation of the remaining nanonode components to bring out the best performance of the nanodevice and ensure its proper functioning in terms of sensor data collection, storage, processing and communication. However, carrying out these functions is not an easy task, due to the fact that the available area in the nanonode is tightly restricted, and therefore, the resources available to the nanoprocessor will also be rather limited. This concern was extensively discussed and analyzed in [6], concluding that the SiGe-based transistor (with $7 \mathrm{~nm}$ technology) is the best technological option nowadays to obtain a functional nanoprocessor that satisfies the expected nanonode requirements in both size and energy consumption. Specifically, its power consumption, as analyzed, is estimated to be $140 \mathrm{nW}$ for a processor working at $500 \mathrm{kHz}$. This operating frequency is enough to accomplish the aforementioned required tasks while maintaining the condition of very low energy consumption.

4.1.2. Memory nanomodules

Diverse types of memories are encompassed in the nanonode; each one of them intended to store information according to different purposes. Firstly, to read/write data rapidly, a RAM (Random Access Memory) module is included. Since the physical position of the data to be read or written is irrelevant, it makes RAM an appropriate solution for cache memories, where data handled by the processor will be temporarily stored. Analyzing the complete RAM family, the technological solution that best fits our particular needs is the A-RAM memory, which combines the advantages of dynamic (DRAM) and static (SRAM) memories [21]. However, due to its volatile nature, A-RAM requires a continuous refreshing signal to retain the data. Secondly, to store permanent data, such as the preset software to boot the nanodevice, a ROM module is also required. For this purpose, NOR flash memory is the best choice, since it assures low read access times, preserving an acceptable bit density and restrained energy consumption when reading. Regarding their power consumption, as reported in [6], the consumption of RAM and ROM modules is included in the value given for the nanoprocessor.

4.1.3. Radiocommunication nanosystem

A radiocommunication nanosystem is required to allow the nanonode to communicate, via EM waves, with the nanorouter or other nanonodes. The first component to deal with in our radiocommunication system is the antenna. At the nanoscale, traditional metallic antennas are not a feasible solution, since they must radiate in the range of hundreds of $\mathrm{THz}$, entailing huge channel attenuation and thus, extremely short transmission coverage. Going beyond this traditional technology, research on other nanotechnological areas has led to the choice of graphene as the best material for the design and development of viable nanoantennas, able to furnish EM nanocommunications [22]. The key advantage of graphene nanoantennas is the ability to propagate surfaceplasmon-polariton (SPP) waves at lower frequencies than noble metals [23]. This behavior allows graphene antennas with the size of a few $\mu \mathrm{m}$ to resonate in the range of 1 to $10 \mathrm{THz}$, which involves resonant frequencies two orders of magnitude lower than those in a nanoscale metal antenna. Moreover, a terahertz transceiver must be integrated into the nanonode in order to feed and drive the graphene nanoantenna. The terahertz transceiver proposed in [24], which was specifically designed to be integrated in a nana graphene-based plasmonic nanotransceiver. The former modulates an electric signal, which carries the bit stream to be sent, while the latter transforms the electric signal into an SPP wave at terahertz frequencies. Thus, the complete radiocommunication nanosystem is composed of the two blocks of the transceiver along with a graphene patch nanoantenna.

Concerning energy consumption, the proposed TS-OOK modulation is based on the use of ultra-short pulses in order to reach a high-power value using a slight amount of energy. Under this premise, the communication system is able to inject $100 \mathrm{pJ}$ in a pulse of $100 \mathrm{fs}$, entailing a power transmission of $60 \mathrm{dBm}$. As was previously calculated, the total path loss at the SDP is $124 \mathrm{~dB}$. Both values (power transmission and path loss) are employed to derive the received power at the receiver, which is $-64 \mathrm{dBm}$. This is an acceptable value to fulfill the EM communication. The use of these femtopulses is feasible due to the very large available bandwidth (1 $\mathrm{THz}$, ranging from 0.5 to $1.5 \mathrm{THz}$ ). Besides, in comparison with the transmission of pulses, the radiocommunication nanosystem will consume less energy in receiving a pulse from the nanorouter. So considering the energy consumption values for a nanodevice proposed in [2], we adopt a consumption of $0.1 \mathrm{pJ}$ per pulse received.

\subsubsection{Nanosensor}

New physical, chemical, and biological nanosensors have been developed by employing graphene and other nanomaterials in 


\section{CHAPTER 3. A NANO COM. NETWORK SCHEME AND ENERGY

their fabrication process [25-28], which contribute novel properties different than the well-known traditional materials. So, nanosensors would identify and measure new events at the nanoscale level, for instance, the physical characteristics of nanostructures, chemical compounds in concentrations as low as one part per billion, or the presence of biological agents such as viruses, or cancerous cells.

Thus, the nanosensor included on the nanonode will ultimately depend on the specific application of the BANN. As each nanosensor will require a particular amount of energy to operate, we assume the generic value employed in [6], that is, $50 \mathrm{nW}$.

\subsection{Nanorouter power consumption}

In the same way as the nanonode, nanorouter specifications are crucial in guaranteeing appropriate performance. As the nanorouter must carry out tasks that require greater computing resources than the nanonode, its physical area should ensure the integration of an energy harvesting system large enough to power its hardware. Under these circumstances, the size envisaged for the nanorouter is $1.3 \times 1.3 \times 0.5 \mathrm{~mm}$, as will be justified in Section 5.2 . Concerning the hardware included in the nanorouter, the layout will be analogous to the nanonode but on a larger scale. This increase in size, along with the increment of computing resources, involves higher energy consumption, which must be adapted to our application scenario.

On the one hand, our estimation for the power consumption of the processor is founded on the following requirements: (i) the nanorouter processor is based on the 7-nm SiGe-based transistor, and therefore, it is larger than the one included in the nanonode, coming close to the size of commercial microprocessors [29], and coming close to the size of commercial microprocessors [29], and
(ii) the operating frequency should ensure suitable performance. Therefore, as an upper limit, we consider that the nanorouter processor will consume the same energy per cycle as the processor built in [29], that is, $2.8 \mathrm{nW}$ per $\mathrm{kHz}$. Regarding the operating frequency, it is set to $5 \mathrm{MHz}$, since it is affordable for the energy harvesting system and should be high enough to provide the additional processing power required to manage communication with a large number of nanonodes and exchange data with the gateway. Thus, the average power consumption of the nanoprocessor including the ROM and RAM modules is $14 \mu \mathrm{W}$.

On the other hand, the radiocommunication system encompassed in the nanorouter would be quite similar to that of the nanonode, since the frequency range covered should be the same in both devices (in the THz band). For the sake of simplicity, in this study, we consider that the nanorouter employs the same modulation scheme as the nanonode (TS-OOK), with a pulse duration and energy which have been set to $100 \mathrm{fs}$ and $100 \mathrm{pJ}$, respectively. Regarding the transmission pulse rate, we think it is reasonable that can support. According to the previously mentioned specifications for the nanonode, its nanoprocessor runs at $500 \mathrm{kHz}$, which should for the nanonode, its nanoprocessor runs at $500 \mathrm{kHz}$, which should TS-OOK modulation ( 1 bit per EM pulse) [18]. Considering this reception rate, the nanorouter should send a pulse every $2 \mu \mathrm{s}$.

\section{Energy harvesting systems}

At the nanoscale, traditional operations like the recharging or replacement of a battery are unfeasible, which leads us to look for new ways to appropriately power the nanodevice components. Nanogenerators are the most widely accepted technological solution by the scientific community [2,3], and in particular, those fabricated using Zinc Oxide ( $\mathrm{ZnO}$ ) nanowires. $\mathrm{ZnO}$ nanowires are one of the most promising systems for energy harvesting [30], since they are able to convert mechanical strains and vibrations into electric energy due to their piezoelectric properties. To this end, nanowires are vertically arranged and firmly fixed at one end to a substrate while the other end is free, thus allowing them to bend. Under this scenario, a voltage and current are induced and collected by both electrodes (the ends of the nanowire). Once the voltage is adjusted (since the potential difference has an opposite sign depending on the nanowire movement), the energy produced is stored in a nanocapacitor to later be employed in the feeding of the nanodevice components.

In light of the network structure described in the introduction, the nanorouter will only be fed by ultrasound waves, while the nanonodes will be powered through their movement in blood flow, except for those in the acting range of ultrasounds. Thus, two different harvesting cases are studied: (i) energy harvesting from blood flow, and (ii) energy obtained from an external source through ultrasound waves.

\subsection{Energy harvesting from the bloodstream}

In the scenario proposed in this work, nanonodes are deployed into the blood flow of a human body to form a nanonetwork. Thi working environment acts on a regular basis; the strain intensity depends on the blood pressure and the frequency of the heartbeat. Under these regular conditions, the average power density produced by a $\mathrm{ZnO}$ nanogenerator reaches a value of $0.01 \mathrm{pW} / \mu \mathrm{m}^{3}$ with a peak voltage of $0.7 \mathrm{~V}$ [31]. This value was obtained unde laboratory conditions when a regular strain at a frequency of $0.3 \mathrm{~Hz}$ was applied. In our case, the heartbeat has a minimum frequency of $1 \mathrm{~Hz}$ which would triple the average power produced. However, we assume the worst case scenario since the energy harvested depends on different factors that require more exhaustive study, such as the position of the nanodevice in the vein, blood pressure or the distance to the heart. Thus, adopting the nanonode dimension suggested in [6], the nanogenerator volume is $128 \mu \mathrm{m}^{3}$, so the envisioned average power produced by each nanonode when they are excited just by blood flow movement is $1.28 \mathrm{pW}$.

\subsection{Energy harvesting from an external source}

Concerning our proposal, the nanorouter is only powered by an external source through ultrasounds. This technological solution resolves two main concerns. Firstly, the nanorouter is placed in the epidermis tissue which tightly restricts their movement and, therefore, their energy harvesting capacity. Secondly, the nanorouter requires significantly more energy to work than nanonodes, since (i) the nanorouter components are larger in size and clearly more powerful and a high amount of energy to feed their components is needed, and (ii) their operating periods are longer than those for the nanonodes.

In order to accurately estimate the power generated by ultrasounds, we follow the methodology proposed in [11] since it fits our case study. As a starting point, we set the ultrasound intensity $\left(I_{0}\right)$ transmitted by the external source, which is restricted to a maximum of $720 \mathrm{~mW} / \mathrm{cm}^{2}$ by medical recommendations. Thus, we consider this value in order to maximize the energy harvesting. As the ultrasound signal must propagate through different biological tissues to reach nanodevices, the attenuation experienced must be taken into account. In particular, two different physical phenomena should be accounted for: (i) the absorption of energy caused by the tissues, which is converted into thermal energy, and (ii) the reflection when the tissue changes.

Similar to absorption losses in EM waves, ultrasound absorption is related with the absorption coefficient of the tissue $(\alpha)$ by means of the following expression:

$\frac{I_{a b s}}{I_{0}}=1-10^{-(\alpha f d / 10)}$ 


\section{CHAPTER 3. A NANO COM. NETWORK SCHEME AND ENERGY MODEL FOR A HUMAN HAND SCENARIO}

where $I_{a b s}$ and $I_{o}$ are the absorbed and transmitted ultrasound intensities, respectively, $f$ is the ultrasound frequency in $\mathrm{MHz}$, and $d$ is the distance in $\mathrm{cm}$. The ultrasound absorption coefficient values are shown in Table 2, extracted from [32]

The energy reflected at tissue interfaces is generated by the difference between their acoustic impedances. The greater the difference, the greater the amount of energy reflected. The mathematical relation between the reflected intensity $\left(I_{r}\right)$ and the incident intensity $\left(I_{0}\right)$ in an interface between two different materials is defined as:

$\frac{I_{r}}{I_{0}}=\frac{\left(Z_{2}-Z_{1}\right)^{2}}{\left(Z_{2}+Z_{1}\right)^{2}}$

where $Z_{1}$ and $Z_{2}$ are the acoustic impedances of both materials. In the case under study, there are three well-defined interfaces: air-skin, skin-fat, and fat-blood. The acoustic impedance of air is 429 Rayl $\left(\mathrm{kg} \mathrm{s}^{-1} \mathrm{~m}^{-2}\right)$, while the acoustic impedance of each biological tissue is indicated in Table 2, also extracted from [32]. As can be seen, in the interface air-skin the large difference between acoustic impedances leads to an excessively high amount of energy reflected (99.91\%). Therefore, the ultrasound source must touch the skin to avoid the air-skin interface and allow the ultrasound signal to directly propagate through biological tissues. In the remaining two interfaces the energy reflected is much lower $(3.28 \%$ and $0.85 \%$, respectively), due to the similarity between their acoustic impedances.

Bearing in mind these two phenomena as well as the layered channel composition, the ultrasound intensity arriving at the receiver $\left(I_{r x}\right)$ is determined by expression (13):

$I_{r x}=I_{o} 10^{-(\alpha f d / 10)}\left(1-\frac{\left(Z_{2}-Z_{1}\right)^{2}}{\left(Z_{2}+Z_{1}\right)^{2}}\right)\left(1-\frac{\left(Z_{3}-Z_{2}\right)^{2}}{\left(Z_{3}+Z_{2}\right)^{2}}\right)$

where $I_{0}$ is the intensity transmitted by the external ultrasound source $\left(720 \mathrm{~mW} / \mathrm{cm}^{2}\right), \alpha$ is the absorption coefficient in $\mathrm{dB} /(\mathrm{cm}$ $\mathrm{MHz}), f$ is the ultrasound frequency in $\mathrm{MHz}, d$ is the distance in $\mathrm{cm}, Z_{1}, Z_{2}$, and $Z_{3}$ are the acoustic impedances of the tissues in our model, i.e. skin, fat, and blood.

Once the ultrasound intensity at the receiver has been analytically obtained, the electric power generated by the piezoelectric generator per unit of area $\left(P_{\text {harv }}\right)$ is calculated as follows:

$P_{\text {harv }}=I_{r x} \gamma$

where, $\gamma$ is the piezoelectric conversion factor. According to the work in [11] and the experimental data from [33], this piezoelectric conversion factor is between 0.0055 and 0.008 with a density of 20 nanowires per $\mu \mathrm{m}^{2}$. In our study, we assume the worst case to make the nanonetwork as robust as possible, that is, only the $0.55 \%$ of the mechanical energy arriving the nanogenerator is transformed into electrical energy.

At this point, the only parameter to be set is the ultrasound frequency $(f)$. From (11), we observe that the frequency impacts on the absorption attenuation, so that higher frequencies will entail a higher absorption by the tissue. However, as the tissue thickness is rather low in the scenario under consideration, the energy absorbed is negligible for the nanorouter (less than $1 \%$ of the ultrasound energy is absorbed at an ultrasound frequency of 1 $\mathrm{MHz}$ ), and considerably low in the case of the nanonode ( $0.06 \%$ of the energy lost at $10 \mathrm{kHz}$ and $5.43 \%$ at $1 \mathrm{MHz}$ ). Consequently, the ultrasound frequency might vary within a wide range (from $10 \mathrm{kHz}$ to $1 \mathrm{MHz}$ ) without incurring high absorption losses. Thus, we set the ultrasound frequency to $250 \mathrm{kHz}$, which is also employed in [34] to design a piezoelectric energy harvester for biomedical implants.

Using all the values mentioned above together with the physical parameters of the tissues specified in Table 2, the power harvested per unit of area is solved from (14) for the nanorouter (39.52 $\left.\mathrm{pW} / \mu \mathrm{m}^{2}\right)$, and for the nanonode ( $\left.37.45 \mathrm{pW} / \mu \mathrm{m}^{2}\right)$.

The requirement that fundamentally affects the determination of the nanorouter size is the amount of harvested energy which, in turn, is influenced by the power consumed. In addition, another important aspect which must be taken into consideration in determining the nanorouter size is related to the time periods in which the nanorouter is transmitting and, therefore, consuming energy. During these periods, among other functions, the nanorouter must drive nanonodes, through instructions dispatched via EM communication, and collect data from them. Since the amount of nanonodes should be huge due to their extremely limited transmission range, the nanorouter energy system must provide enough power to allow uninterrupted data transmission to/from all of them. This is the reason why the nanorouter must always remain active. Moreover, being continuously powered, communication with the gateway, which is not addressed in this work, should not affect energy consumption.

Keeping these premises in mind, the processor and memories in the nanorouter would consume $14 \mu \mathrm{W}$, while the radiocommunication system would transmit, at a maximum, 500000 pulses per second (this is the value of the nanoprocessor frequency). Since the energy per pulse is $100 \mathrm{pJ}$ and the time between pulses is $2 \mu \mathrm{s}$, the average power radiated is $50 \mu \mathrm{W}$. As we can observe, the total power consumed by the nanorouter is $64 \mu \mathrm{W}$. To feed the device continuously, the power generator must harvest this power from the external source. So, as the power harvesting harvested per unit of area is valued at $39.52 \mathrm{pW} / \mu^{2}$, a nanorouter area of $1.66 \mathrm{~mm}^{2}$, (e.g. a square of $1.3 \times 1.3 \mathrm{~mm}$ ) must be enough to guarantee nonstop operation. Its thickness is not limited by the generator, since the average length of $\mathrm{ZnO}$ nanowires is a few micrometers. Thus, we consider a conservative value of thickness of $0.5 \mathrm{~mm}$.

Likewise, repeating the aim announced at the beginning of this subsection, the nanonode will harvest energy from ultrasounds when it is within the range. According to its nanogenerator area $\left(64 \mu \mathrm{m}^{2}\right)$, the power scavenged by the nanonode from the external source should be $2.40 \mathrm{nW}$, as calculated in [6].

\section{Nanonetworking energy model and communication scheme}

As mentioned before, the nanonode should accurately know when to wake up to carry out the transmission/reception tasks due to: (i) the restricted amount of energy available, and (ii) excessive path loss when it is not aligned with the nanorouter. In our transmission scheme, the recharging of the capacitors of the nanonodes relies on their movement in blood flow along with the energy harvested from ultrasound. Employing these recharge modes, nanonodes should harvest the required energy to accomplish the transmission of a complete data packet. Then, when they approach the nanorouter and enter the ultrasound region, they should wait to get as close as possible to the SDP to ensure minimum path loss and dispatch the sensor data to the nanorouter.

Under these premises, in this section we derive an energy model that makes the EM communication between nanorouter and nanonode feasible. Firstly, we determine a suitable packet size to face these energy storage restrictions. Secondly, we calculate the time length that the nanonode should remain asleep once it enters the ultrasound region. Finally, we define the complete energy model for the BANN.

\subsection{Packet size}

Bearing in mind the volumetric capacitance of the hybrid supercapacitor $\left(1100 \mathrm{~F} / \mathrm{cm}^{3}\right)$ proposed in [35], the total capacitance of the nanocapacitor integrated in the nanonode is $70.4 \mathrm{nF}$. Using the well-known expression, $E_{\max }=\frac{1}{2} C V_{g}^{2}$, for the generator voltage 


\section{CHAPTER 3. A NANO COM. NETWORK SCHEME AND ENERGY

$\left(V_{g}\right)$ of $0.7 \mathrm{~V}$, the maximum energy stored in the nanocapacitor is $17.24 \mathrm{~nJ}$. As each bit is transmitted in an individual pulse and each pulse requires an amount of energy equal to $100 \mathrm{pJ}$, the maximum packet size that the nanodevice can transmit for a single active cycle is 172 bits. As can be seen, the maximum energy radiated per pulse should be around $100 \mathrm{pJ}$ to ensure an acceptable packet length. Otherwise, if higher energy per pulse is used, the bit stream that the nanonode can support with a single charge would be shorter and, therefore, inadequate to design a robust communication scheme for BANN.

Therefore, taking into account the trade-off between recharge time and communication robustness, we proposed a packet size of 40 bits ( 5 bytes). If a higher packet size is used, the energy harvesting time would be excessively long. In this way, the first 23 bits comprise the header. This header contains the ID of each nanonode ranging from 0 to 8388607 , so it encompasses enough IDs to identify each one of the massive number of nanonodes envisaged for a nanonetwork. This range can be divided into subranges to identify different types of nanonodes in the BANN. For instance, according to the biological magnitude to measure, IDs from 0 to 4194304 would correspond to temperature nanonodes, and from 4194304 to 8388607 would be reserved for glucose nanonodes. Thus, the nanonetwork can collect and process different biological samples. Furthermore, an ID must be assigned to the nanorouter. The next 16 bits are reserved for the payload, that is, the data gathered by the nanonode. In this way, each sample (analog sensor information collected by a nanosensor to later be processed and converted into digital data) can be read with a resolution of 65536 levels, enough for the measurement of medical parameters. Finally, the last bit is a parity bit to make the communication more trustworthy and robust.

\subsection{Nanonode waiting time}

In order to minimize energy consumption, the nanonode remains in sleep mode whilst its nanogenerator is harvesting and storing energy from blood flow. Nevertheless, as soon as a nanonode, charged with enough energy to transmit, detects a sudden ode, charged with enough energy to transmit, detects a sudden
increase in the charge level (this indicates that it has entered the increase in the charge level (this indicates that it has entered the switch to reception mode. In this mode, the nanonode activates the transceiver to listen to the physical medium. This waiting time is set to ensure that: (i) the energy harvested from ultrasounds is equal to the energy consumption in reception mode, and (ii) the reception takes place near to the SDP. Otherwise, the time required to charge the nanocapacitor for communication tasks would increase unnecessarily.

As stated in the study [36], the nanonode arrives at the ultrasound region at a speed of $7.1 \mathrm{~cm} / \mathrm{s}$. Thus, the nanonode takes approximately $70 \mathrm{~ms}$ to reach the SDP. As mentioned above, the nanonode generator is able to harvest $2.40 \mathrm{nW}$ from ultrasounds, no the theoretical maximum energy gathered during this period is $0.168 \mathrm{~nJ}$. Since the nanotransceiver consumes $0.1 \mathrm{pJ}$ per pulse received and the nanorouter radiates a pulse every $2 \mu \mathrm{s}$, the average power consumption of the radiocommunication system will be $50 \mathrm{nW}$ in reception mode. Thus, it can be activated during 3.36 ms to be exclusively fed by ultrasounds. During this short time interval, the nanorouter is able to send a maximum of 1680 bits, which is enough to send basic instructions. Hence, the nanonode stays asleep for a waiting time of $66.64 \mathrm{~ms}$ after the detection of ultrasounds.

\subsection{Communication schedule}

After defining and calculating all the parameters employed in the energy model, in this subsection we describe the transmission schedule for our application scenario. On the one hand, the nanonodes are moving through the human body harvesting energy and recharging their nanocapacitors to store the energy required to send an entire packet. This means the charging time $\left(t_{\text {charge }}\right)$ relies on: (i) the nanonode energy consumption, which includes the power consumption of all nanonode components, and (ii) the power harvested from the environment $\left(P_{h}\right)$. Assuming the wors case scenario, (all the bits sent are $1 \mathrm{~s}$ ) and that one bit is dispatched in each nanoprocessor clock cycle, the energy consumption to transmit a packet $\left(E_{p}\right)$ is the following:

$E_{p}=\frac{n_{p}}{f_{n p}}\left(P_{n p}+P_{s}\right)+n_{p} E_{p u l s e}$

where $n_{p}$ is the number of bits in a packet, $f_{n p}$ stands for the nanoprocessor operating frequency, $P_{n p}$ is the power consumed by both nanoprocessor and memories, $P_{S}$ is the power consumed by the nanosensor, and $E_{\text {puse }}$ is the energy radiated per pulse. As the time required for the nanosensor to capture a sample depends on the type of application, we have taken as an assumption that the nanosensor will be active during the whole transmission time $\left(t_{t x}\right)$. Therefore, since $E_{p}$ should be equal to the energy harvested during $t_{\text {charge }}$, it can be obtained by this expression:

$t_{\text {charge }}=\frac{E_{p}}{P_{h}}=\frac{1}{P_{h}}\left(\frac{n_{p}}{f_{n p}}\left(P_{n p}+P_{s}\right)+n_{p} E_{\text {pulse }}\right)$

As was determined in previous sections, the value of each parameter is as follows: $P_{h}=1.28 \mathrm{pW}, n_{p}=40 \mathrm{bits}, f_{n p}=500 \mathrm{kHz}$, $P_{n p}=140 \mathrm{nW}, P_{s}=50 \mathrm{nW}$, and $E_{\text {pulse }}=100 \mathrm{pJ}$. Using these values, $t_{\text {charge }}$ is approximately $52 \mathrm{~min}$. A priori, this value seems too high, but bearing in mind that millions of nanonodes may be deployed in a BANN (in our case, if the highest ID is 8388607 , this value will be the maximum number of nanonodes), the entire nanonetwork could take a biological measurement every few seconds. It is noteworthy that we consider that the nanonode harvests all its energy from blood flow and just crosses the ultrasound region when it has stored enough energy to send a packet. However, during $t_{\text {charge, }}$, if the nanonode could cross the ultrasound region without a suitable energy level, it would not switch to reception mode and would take advantage of this extra energy, which would reduce the time required to recharge the nanocapacitor.

All in all, each nanonode should be flowing into the bloodstream for at least $52 \mathrm{~min}$ in order to accomplish the communication with the nanorouter. When the nanogenerator gathers enough energy to transmit a packet, the nanonode waits until it detects ultrasounds. Once the nanonode perceives it is within the ultrasound zone, it remains in sleep mode for $66.64 \mathrm{~ms}$. Then it changes to reception mode to receive EM pulses from the nanorouter. After $3.36 \mathrm{~ms}$ in this state (which assures the reception of packets up to 1680 bits, allowing for a comfortable reception of control information), the nanonode transmits its entire packet ( 40 bits), in a transmission time $t_{t x}$ equal to $80 \mu \mathrm{s}$. The communication scheme is indicated in Table 3, and the energy available in the nanodevice during the communication procedure is shown in Fig. 6. Following this procedure, the energy stored in the capacitor reaches a maximum of $4.168 \mathrm{~nJ}$ which guarantees the reception and transmission of information with the nanorouter in the SDP surroundings.

\section{Conclusions}

In this paper, a BANN communication scheme has been realistically and accurately designed to enable the monitoring of, for instance, medical parameters between nanonodes and a nanorouter, thus overcoming the huge path loss and molecular absorption suffered in the human body. To face these limitations and show the feasibility of our BANN communication scheme, a specific human body scenario, i.e. the dorsum of the hand, has been selected. The 


\section{CHAPTER 3. A NANO COM. NETWORK SCHEME AND ENERGY MODEL FOR A HUMAN HAND SCENARIO}

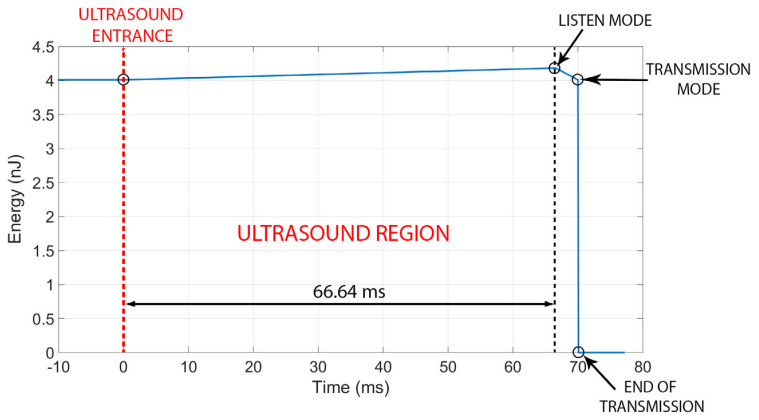

Fig. 6. Nanonode energy level during communication with the nanorouter.

Table 3

\begin{tabular}{lll}
\hline \multicolumn{2}{l}{ Communication timing schedule. } & \\
\hline State & Consumption $(\mu \mathrm{W})$ & Time \\
\hline Blood flow & & \\
\hline Sleep & $\sim 0$ & $>52 \mathrm{~min}$ \\
\hline Ultrasound region & & \\
\hline Sleep & $\sim 0$ & $66.64 \mathrm{~ms}$ \\
Listen & 0.05 & $3.36 \mathrm{~ms}$ \\
Transmission & $50^{\mathrm{a}}$ & $80 \mu \mathrm{s}$ \\
\hline
\end{tabular}

$50 \mu \mathrm{W}$ is the average transmission power, calculated as: $E_{p} / t_{t x}$.

electrical properties for its different biological tissues at $1 \mathrm{THz}$, which is the envisaged operational frequency for nanodevices have been considered. Unlike other BANN works, the adjusted thickness of the biological tissues of the hand (see Table 1) facilitates the of the biological tissues of the hand (see Table 1) facilitates the calculation of the propagation losses. However, a huge amount of power is still required to transmit data over such short distances
(a few millimeters). Therefore, the energy management required has also been analyzed and evaluated to ensure the appropriate operation of the BANN; the power consumption of each type of nanodevice proposed, as well as their energy harvesting capacity have been thoroughly derived.

Going into detail, nanonodes (in the sleeping mode) are recharged by means of: (i) the bloodstream itself, and (ii) an external ultrasound system. The nanorouter is continuously fed by the external ultrasound source system. Once the capacitor of the nanonodes has enough energy stored $(4.168 \mathrm{~nJ})$, the nanonodes will exchange control information and sensor acquired data with the nanorouter in the SDP surroundings. Employing this communication scheme, each nanonode is able to gather and transmit a packet ( 40 bits) to the nanorouter every $52 \mathrm{~min}$. However, taking into account the extremely high number of them expected (a maximum of 8388607 ), the monitoring of a medical parameter will be almost constant. Although the focus of this work has been on nanonode-nanorouter communication, an outline of the interconnexion of the BANN with an external device has been also described. The study of a communication model between the BANN and external devices to dispatch sensor data to end users is left for future work

\section{Acknowledgments}

This work has been supported by the project AIM, ref. TEC201676465-C2-1-R (AEI/FEDER, UE). Sebastian Canovas-Carrasco also thanks the Spanish MECD for an FPU (ref. FPU16/03530) predoctoral fellowship.

\section{References}

1] K. Yang, A. Pellegrini, M.O. Munoz, A. Brizzi, A. Alomainy, Y. Hao, Numerical analysis and characterization of $\mathrm{THz}$ propagation channel for body-centric nano-communications, IEEE Trans. Terahertz Sci. Technol. 5 (2015) 419-426. http://dx.doi.org/10.1109/TTHZ.2015.2419823.

[2] J.M. Jornet, I.F. Akyildiz, Joint energy harvesting and communication analy sis for perpetual wireless nanosensor networks in the terahertz band, IEE Trans. Nanotechnol. 11 (2012) 570-580. http://dx.doi.org/10.1109/TNANO. 2012.2186313.

. stack for body area nano-networks, Nano Commun. Netw. 6 (2015) 74-84.

4] B. Liu Y Z Zhang X Jiang Z Wu An energy-efficient d

Bollection scheme in CANDAR 2015, 2016, pp. 240-245. http://dx.doi.org/10.1109/CANDAR.2015. 66.

[5] J.M. Jornet, J. Capdevila Pujol, J. Solé Pareta, PHLAME: A physical layer aware MAC protocol for electromagnetic nanonetworks in the Terahertz band, Nano Commun. Netw. 3 (2012) 74-81. http://dx.doi.org/10.1016/j.nancom.2012.01. 006.

[6] S. Canovas-Carrasco, A.-J. Garcia-Sanchez, F. Garcia-Sanchez, J. Garcia-Haro Conceptual design of a nano-networking device, Sensors 16 (2016) 2104. http //dx.doi.org/10.3390/s16122104

7] M. Pierobon, J.M. Jornet, N. Akkari, S. Almasri, I.F. Akyildiz, A routing framework for energy harvesting wireless nanosensor networks in the Terahertz Band, $0665-y$

8] J.M. Jornet, I.F. Akyildiz, Channel modeling and capacity analysis for electromagnetic wireless nanonetworks in the terahertz band, IEEE Trans. Wirel.
Commun. 10(2011)3211-3221. http://dxdoi.org/10.1109/TWC 2011.081011. 100545 .

[9] G. Piro, P. Bia, G. Boggia, D. Caratelli, L.A. Grieco, L. Mescia, Terahertz electromagnetic field propagation in human tissues: A study on communication capabilities, Nano Commun. Netw. 10 (2016) 51-59. http://dx.doi.org/10.1016 j.nancom.2016.07.010.

[10] E. Berry, A.]. Fitzgerald, N.N. Zinov'ev, G.C. Walker, S. Homer-Vanniasinkam, C.D. Sudworth, R.E. Miles, J.M. Chamberlain, M.A. Smith, Optical properties of tissue measured using terahertz-pulsed imaging, in: Proc. SPIE Phys. Med. Imaging, 2003, p. 459. http://dx.doi.org/10.1117/12.479993.

[11] M. Donohoe, S. Balasubramaniam, B. Jennings, J.M. Jornet, Powering in-body nanosensors with ultrasounds, IEEE Trans. Nanotechnol. 15 (2016) 151-154. http://dx.doi.org/10.1109/TNANO.2015.2509029.

[12] G.C.R. Melia, M.P. Robinson, I.D. Flintoft, Development of a layered broadband model of biological materials for aerospace applications, in: EMC Eur. 2011 . jsp?.

[13] Y. Lee, K. Hwang, Skin thickness of Korean adults, Surg. Ra
183-189. http://dx.doi.org/10.1007/s00276-002-0034-5.

[14] J.S. Petrofsky, M. Prowse, E. Lohman, The influence of ageing and diabetes on skin and subcutaneous fat thickness in different regions of the body. J. Appl. Res. 8 (2008) 55-61.

[15] A.O. Alradi, S.G. Carruthers, Evaluation and application of the linear variable differential transformer technique for the assessment of human dorsal hand vein alpha-receptor activity, Clin. Pharmacol. Ther. 38 (1985) 495-502. http: //dx.doi.org/10.1038/clpt.1985.214 


\section{CHAPTER 3. A NANO COM. NETWORK SCHEME AND ENERGY}

[16] A.N. Pal, A. Ghosh, Ultralow noise field-effect transistor from multilayer graphene,
3206658.

[17] A.A. Balandin, Low-frequency $1 /$ f noise in graphene devices, Nat. Nanotechnol. 8 (2013) 549-555. http://dx.doi.org/10.1038/nnano.2013.14

[18] J.M. Jornet, I.F. Akyildiz, Femtosecond-long pulse-based modulation for Terahertz band communication in nanonetworks, IEEE Trans. Commun. 62 (2014) 1742-1754. http://dx.doi.org/10.1109/TCOMM.2014.033014.130403.

[19] P. Boronin, V. Petrov, D. Moltchanov, Y. Koucheryavy, J.M. Jornet, Capacity and throughput analysis of nanoscale machine communication through trans-
parency windows in the terahertz band, Nano Commun. Netw. 5 (2014) 72-82. parency windows in the terahertz band, Nano Con
http://dx.doi.org/10.1016/j.nancom.2014.06.001.

[20] I.F. Akyildiz, J.M. Jornet, Electromagnetic wireless nanosensor networks, Nano

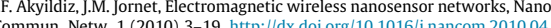
001.

[21] N. Rodriguez, S. Cristoloveanu, F. Gamiz, A-RAM: Novel capacitor-less DRAM memory, in: Proc - IEEE Int. SOI Conf., 2009, pp. 4-5. http://dx.doi.org/10. 1109/SOL. 2009.5318734 .

[22] J.M. Jornet, I.F. Akyildiz, Graphene-based nano-antennas for electromagnetic nanocommunications in the terahertz band, in: Antennas Propag. EuCAP 2010 Proc. Fourth Eur. Conf., 2010, pp. 1-5 http://ieeexplore.ieee.org/xpls/abs_all. jsp?arnumber $=5505569$.

[23] G.W. Hanson, Dyadic green's functions for an anisotropic, non-local model of biased graphene, IEEE Trans. Antennas Propag. 56 (2008) 747-757. http: //dx.doi.org/10.1109/TAP.2008.917005

[24] J.M. Jornet, I.F. Akyildiz, Graphene-based plasmonic nano-transceiver for terahertz band communication, in: 8th Eur. Conf. Antennas Propag., EuCAP 2014,

[25] I. Park, Z. Li, A.P. Pisano, R.S. Williams, Top-down fabricated silicon nanowire sensors for real-time chemical detection, Nanotechnology 21 (2010) 15501.
s.t. sensors for real-time chemical detection, Nanotech
http://dx.doi.org/10.1088/0957-4484/21/1/015501.

[26] N. Shehada, G. Brönstrup, K. Funka, S. Christiansen, M. Leja, H. Haick, Ultrasensitive silicon nanowire for real-world gas sensing. noninvasive diagnosis of cancer from breath volatolome, Nano Lett. 15 (2015) 1288-1295. http://dx.doi.org/10.1021/n1504482t.

[27] V. Sorkin, Y.W. Zhang, Graphene-based pressure nano-sensors, J. Mol. Model. 17 (2011) 2825-2830. http://dx.doi.org/10.1007/s00894-011-0972-0.

[28] P.M. Kosaka, V. Pini, J.J. Ruz, R. a da Silva, M.U. González, D. Ramos, M. Calleja, . Tamayo, Detection of cancer biomarkers in serum using a hybrid mechanical and optoplasmonic nanosensor, Nat. Nanotechnol. 9 (2014) 1047-1053. http: /dx.doi.org/10.1038/nnano.2014.250.

[29] S. Hanson, M. Seok, Y. Lin, Z. Foo, D. Kim, Y. Lee, N. Liu, D. Sylvester, D. Blaauw, low-voltage processor for sensing applications with picowatt standby mode, IEEE J. Solid-State Circuits 44 (2009) 1145-1155. http://dx.doi.org/10.1109/
JSSC.2009.2014205.

301 $S$ X

Nat. Y. Q. Xu, Y. Wei, R. Yang, Z.L. Wang, Self-powered nanowire devices, (2010) 366-373. http://dx.doi.org/10.1038/nnano.2010.

[31] Y. Hu, Y. Zhang, C. Xu, L. Lin, R.L. Snyder, Z.L. Wang, Self-Powered system with wireless data transmissic

[32] H. Azhari, Appendix A: Typical acoustic properties of tissues, in: Basics Biomed. Ultrasound Eng, John Wiley \& Sons, Inc, Hoboken, NJ, USA, 2010. http://dx.doi. org/10.1002/9780470561478.app

[33] Z.L. Wang, J. Song, Piezoelectric nanogenerators based on zinc oxide nanowire arrays, Science 312 (2006) 242-246. http://dx.doi.org/10.1126/science.11240 05.

[34] Q. Shi, T. Wang, C. Lee, MEMS based broadband piezoelectric ultrasonic energy harvester (PUEH) for enabling self-powered implantable biomedical devices,
Sci. Rep. 6 (2016) 1-10. http://dx.doi.org/10.1038/srep24946.
[35] M.F. El-Kady, M. Ihns, M. Li, J.Y. Hwang, M.F. Mousavi, L. Chaney, A.T. Lech, R.B. Kaner, Engineering three-dimensional hybrid supercapacitors and microsupercapacitors for high-performance integrated energy storage, Proc. Natl.

[36] M. Klarhöfer, B. Csapo, C. Balassy, J.C. Szeles, E. Moser, High-resolution bloo flow velocity measurements in the human finger, Magn. Reson. Med. 45 (2001) 716-719. http://dx.doi.org/10.1002/mrm.1096.

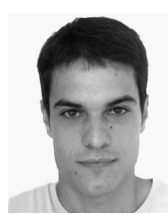

Sebastian Canovas-Carrasco received the B.S. degree in telecommunication systems engineering and the M.S. deree in telecommunications engineering from the Univerrespectively, where he is currently pursuing the $\mathrm{Ph}$. degree. His research interest includes the development of electromagnetic wireless nanonetworks and radiocommunications at the THz band.

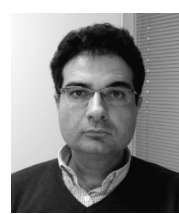

Antonio-Javier Garcia-Sanchez received the Industria Engineering degree (M.S. degree) in 2000 from the Universidad Polltectica de Cartagena (UPCT), Spain. Since 200 he has joined the Department of Information Technologies . the UPCT. He is a (co)author of more thate 50 conferon and journal papers, fifteen of them indexed in the Journa Citation Report (JCR). He has been the main head in several research projects in the field of communication networks and optimization, and he currently is a reviewer of severa journals listed in the ISI-JCR. He is also inventor/co-inventor of 7 patents or utility models and he has been a TPC member or Chair in about thirty Internationa Congresses or Workshops. His main research interests are in the areas of wireles sensor networks (WSNs), streaming services, Smart Grid and soil data collection.

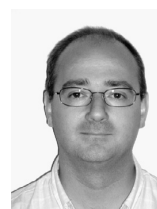

Joan Garcia-Haro received the M.S. and Ph.D. degrees in telecommunication engineering from the Universita 1995. respectively. He is currently a Professor with Unive respectively. He is currenty a Pres) or co-author of more than 70 journal papers mainly in the fields of switching wireless networking and performace evaluation. Prof. Garcia-Haro served as Editor-in-Chief of the IEEE Global Communications Newsletter, included in the IEEE Communications Magazine, from April 2002 to December 2004. He has been Technical Editor of the same magazine from March 2001 to December 2011. He also received an Honorable Mention for the IEEE Communications Society Best Tutorial paper Award (1995). He has been a visiting scholar at Queen's University at Kingston, Canada (1991-1992) and at Cornell University, Ithaca, USA (2010-2011). 
Chapter 4

\section{On the nature of energy-feasible wireless nanosensor networks}




\section{Article}

\section{On the Nature of Energy-Feasible Wireless Nanosensor Networks}

Sebastian Canovas-Carrasco, Antonio-Javier Garcia-Sanchez * and Joan Garcia-Haro

Department of Information and Communication Technologies, Technical University of Cartagena, 30202 Cartagena, Spain; sebas canovas@upct.es (S.C.-C.); joang.haro@upct.es (J.G.-H.)

* Cornespondence: antoniojavier.garcia@upct.es; Tel.: +34-968-326-538

Received: 15 March 2018; Accepted: 24 April 2018; Published: 27 April 2018

Abstract: Electromagnetic nanocommunications, understood as the communication between electronic nanoscale devices through electromagnetic waves in the terahertz band, has attracted increasing attention in recent years. In this regard, several solutions have already been proposed. However, many of them do not sufficiently capture the significance of the limitations in nanodevice energy-gathering and storing capacity. In this paper, we address key factors affecting the energy consumption of nanodevices, highlighting the effect of the communication scheme employed. Then, we also examine how nanodevices are powered, focusing on the main parameters governing the powering nanosystem. Different mathematical expressions are derived to analyze the impact of these parameters on its performance. Based on these expressions, the functionality of a nanogenerator is evaluated to gain insight into the conditions under which a wireless nanosensor network (WNSN) is viable from the energetic point of view. The results reveal that a micrometer-sized piezoelectric system in high-lossy environments (exceeding $100 \mathrm{~dB} / \mathrm{mm}$ ) becomes inoperative for transmission distances over $1.5 \mathrm{~mm}$ by its inability to harvest and store the amount of energy required to overcome the path loss.

Keywords: energy harvesting; nanodevice; piezoelectric generator; terahertz communications; wireless nanosensor networks

\section{Introduction}

Progress in the area of nanotechnology is pushing for new communication techniques to coordinate numerous nanoscale devices. These so-called nanodevices would be able to gather physical parameters at the nanoscale, monitoring and even acting on scenarios yet unexplored. Nanodevices would enable unimaginable applications in many fields such as medicine (e.g., detecting cancerous cells or targeted drug delivery), environmental science (e.g., measurement of atmosphere components) or industry (e.g., critical nanofractures' detection in machines or structures). Nevertheless, owing to their limited size, nanodevice resources are severely limited, which hinders their capacity to perform a stand-alone service. Therefore, the ability to carry out useful applications effectively relies on a cooperative effort among nanodevices, establishing wireless nanosensor networks (WNSN).

Scientific literature reveals how electromagnetic (EM) nanodevices should communicate [1-5], proposing innovative communication techniques for media access control, synchronization or addressing which are adapted to the extreme constraints of nanodevices (low computing resources, transmission in the $\mathrm{THz}$ band, short communication range or high number of nodes, among others) All these works contribute by motivating and suggesting valuable advances in WNSN. However, there is a major limiting factor to be analyzed more thoroughly, energy management, which has a direct impact on the feasibility of an operational WNSN. 


\section{CHAPTER 4. ON THE NATURE OF ENERGY-FEASIBLE WIRELESS NANOSENSOR NETWORKS}

In this regard, authors in [6] proposed the first energy analysis for nanonetworks, introducing a powering nanosystem composed of a piezoelectric nanogenerator and a nanocapacitor. The former converts mechanical energy into electrical while the latter stores the energy generated. Based on this nanosystem, with determined specifications (a size of $1000 \mu \mathrm{m}^{2}$ for both components; 6 pC per charge cycle for the nanogenerator and $9 \mathrm{nF}$ for the nanocapacitor), authors capture the nanonetwork energetic behavior. To this effect, a probabilistic analysis of the network traffic and interferences between nodes is developed to obtain a distribution of the energy available in each node. The energy consumption assumed in [6] for communication is constant and fixed to $1 \mathrm{pJ}$ per pulse $(10 \mathrm{~W})$ for transmission and $0.1 \mathrm{pJ}$ in the reception of a pulse, regardless of the medium in which nodes are deployed.

From a different approach but based on the same powering solution (piezoelectric generator together with a capacitor), this paper aims to investigate the energy scarcity problem in WNSN from a more realistic perspective, analyzing those factors that should be considered in an energy-feasible WNSN. This study is motivated by the high transmission powers that have been assumed by different already published communication schemes [4,7] to overcome the huge path loss at terahertz frequencies when the nanonetwork is deployed in a lossy medium (e.g., the human body). In many cases, these transmission powers might be unaffordable for nanodevices. To this end, we focus on the impact of tuning a set of different factors on the behavior of a nanonetwork, not being constrained to specific values of, for instance, the area or transmission energy treated in isolation, unlike [6]. We also evaluate how the increase in both energy (power) transmission and path loss jeopardize the feasibility of a WNSN. In addition, the influence of different key parameters involved in a WNSN (such as generator and capacitor area, energy source, or particular technology employed) are also analyzed towards the design of a proper and feasible solution depending on the application. In this manner, the paper's organization and its main contributions can be summarized as follows.

In Section 2, we explore and synthesize the main issues impacting on the energy consumption in WNSN. First, we evaluate the influence of the energy devoted to communication compared to the total energy consumption in a nanodevice. The power transmission values considered have been employed in the related literature. Second, a straightforward communication scheme is proposed to illustrate numerically the number of pulses, and thus the energy consumption, required to conduct a realistic communication between two nanodevices. Finally, we review three conceivable network topologies proposed in diverse works, summarizing their strengths and limitations from the energetic point of view.

In Section 3, we concentrate upon the most accepted solution to power nanodevices, based on a piezoelectric generator, highlighting its main features. Then, we analyze the three principal factors impacting on the powering nanosystem performance (area, technology, and energy source). To this effect, different mathematical expressions are derived for each factor. Results obtained lead to relevant conclusions to be considered in future nanodevice and WNSN designs.

Section 4 covers the study of the feasibility of WNSN from an energetic point of view, deriving mathematical expressions to link energy consumption with powering factors. The results provide insight into the communication characteristics of feasible EM-based WNSN. Finally, Section 5 concludes the paper.

\section{Energy Consumption in Wireless NanoSensor Networks (WNSN): Key Factors}

There are several factors impacting on the energy consumption of EM-based WNSN, which should be properly tuned depending on the application. In this section, we identify and review the most important, highlighting their main features.

2.1. Transmission Power

The dominant communication mechanism in EM-based WNSN is the so-called Time Spread On-Off Keying (TS-OOK) modulation [3], which employs a pulse to transmit a logical " 1 " and the logical " 0 " is sent as silence. The time duration of each pulse is set to 100 femtoseconds, although the 


\section{CHAPTER 4. ON THE NATURE OF ENERGY-FEASIBLE WIRELESS}

energy per pulse $\left(E_{\text {pulse }}\right)$ varies for each work in the related literature, leading to different transmission powers. These proposed transmission powers $\left(P_{t x}\right)$ fluctuate from $1 \mu \mathrm{W}$ to $5 \mathrm{~kW}$, that is, from 1 aJ to $500 \mathrm{pJ}$ per pulse [4,5,7-12]. Low power values are used for WNSN deployed in low-loss environments (e.g., the air) and micro-range communications (which depends on network topology). Conversely, whether the medium presents high water content (e.g., biological tissues) or the communication range increases (few millimeters), greater power values are required to overcome the high path loss resulting from working at $\mathrm{THz}$ radiation frequencies. Although the pulse duration is extremely short, these transmission powers entail significant energy consumption when compared to the energy consumption of the remaining components of the nanodevice denoted as $E_{d e v}$, which can be expressed as $E_{d e v}=P_{d e v}$ $t_{o n}$, being $P_{d e v}$ the power consumed by the non-communicating parts of the nanodevice when it is active during a period $\left(t_{o n}\right)$. For the sake of clarity, in Figure 1, the transmission power is compared to the total nanodevice power consumption $\left(P_{\text {total }}\right)$, including all the nanodevice components (processor, memories, buffers, sensor, and radiocommunication system): $P_{t o t a l}=P_{t x}+P_{d e v}$. The value of $P_{d e v}$ is estimated to be approximately $240 \mathrm{nW}$ [13].

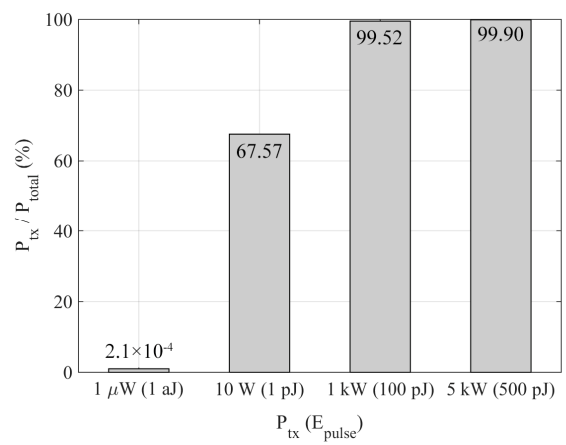

Figure 1. Relation between power required for communication and the total power consumption for different transmission powers.

As can be observed, for a low transmission power the percentage of power with respect to the total is lower than $1 \%$, whereas for a higher transmission power this value rises to $99 \%$. In the latter case, communication among nanodevices poses a real challenge that must be carefully analyzed when developing a communication protocol for WNSN.

\subsection{Pulses Transmitted with a Single Charge}

As mentioned, the EM communication envisioned for WNSN is based on the exchange of electromagnetic pulses. Thus, the total energy required to conduct the transmission of a particular amount of information $\left(E_{t x}\right)$ is determined by the number of pulses $\left(n_{p u l s e}\right)$ radiated during the entire packet exchange: $E_{t x}=E_{\text {pulse }} n_{\text {pulse }}$. As an example, let us suppose a straightforward communication scheme, similar to the protocol proposed in [4], for a WNSN intended to measure blood cholesterol. The communication starts when a nanodevice sends a transmission request packet, containing 24 bits for synchronization, 48 bits devoted to transmitter and receiver addresses, and 8 bits of checksum to detect transmission errors. Upon the acknowledgement packet reception, the transmitter begins the transmission of a data packet. It includes the same fields as the transmission request packet together with the useful data (payload). The number of bits assigned to the payload will ultimately depend on the application for which the WNSN is envisioned. In this case, cholesterol levels in a human body 


\section{CHAPTER 4. ON THE NATURE OF ENERGY-FEASIBLE WIRELESS NANOSENSOR NETWORKS}

usually range from $20 \mathrm{mg} / \mathrm{dL}$ to $300 \mathrm{mg} / \mathrm{dL}$. Thus, in order to cover the entire range with a sensor resolution of $1 \mathrm{mg} / \mathrm{dL}$, the data length must be 9 bits or more, leading to a data packet length of 89 bits.

Thereby, the complete communication consists of 169 bits ( 80 for the transmission request packet and 89 for the data packet), so the transmitter nanodevice must be able to send at least 85 pulses with one single charge, considering that bits ' 1 ' and ' 0 ' are equally distributed (probability of sending a pulse ( $p_{\text {pulse }}$ ) equal to 0.5$)$. Even though this value might seem easily affordable, it is an important concern for nanodevices when the energy per pulse becomes too high, as we will further discuss We should note that low-weight codes for WNSN have been proposed to optimize the probability of sending a ' 0 ' and then reduce the number of pulses transmitted [14]. However, this optimization depends on the channel and network conditions, so for the sake of simplicity, an equitable distribution of ' 1 ' and ' 0 ' has been considered.

\subsection{Network Topology}

Network topology impacts on two key issues: the distance between nanonodes and the communication techniques required. In this sort of network, the EM path loss reaches huge values due to the absorption loss operating in the THz band. In high-lossy mediums (e.g., blood), path loss can reach values up to $120 \mathrm{~dB}$ per millimeter [15]. Thus, the distance between nanodevices belonging to the WNSN is a crucial factor that determines the transmission power needed to ensure an acceptable power density at the receiver. For instance, a small difference of $0.5 \mathrm{~mm}$ entails $60 \mathrm{~dB}$ of attenuation, which is barely enough to cut off the communication. On the other hand, the communication protocols employed in a WNSN will be subject to the network topology, which directly affects the energy consumption. To illustrate both questions, the most common topologies proposed in the literature for WNSN are reviewed, tackling their routing scheme from the energy consumption viewpoint. Table specifies the main strengths and limitations of each network topology.

Table 1. Strengths and limitations for each network topology.

\begin{tabular}{|c|c|c|}
\hline Network Topology & Strengths & Limitations \\
\hline Cluster-based & $\begin{array}{l}\text { Routing complexity falls on } \\
\text { the nanocontroller } \\
\text { - } \quad \text { Address reusability }\end{array}$ & $\begin{array}{l}\text { Problems if the wireless nanosensor network } \\
\text { (WNSN) is not static }\end{array}$ \\
\hline Mesh & $\begin{array}{ll}\text { - } & \text { Simplicity using flooding } \\
\text { - } & \text { All nanodevices are identical } \\
\text { - } & \text { Able to cover a volume or surface }\end{array}$ & $\begin{array}{l}\text { - Flooding implies excessive retransmissions, } \\
\text { whereas other routing schemes are complex } \\
\text { to implement. } \\
\text { - } \quad \text { Routing complexity falls on nanonodes } \\
\text { - } \quad \text { High energy consumption }\end{array}$ \\
\hline Infrastructure-based & $\begin{array}{ll}\text { - } & \text { Routing complexity falls on } \\
\text { the nanorouter } \\
\text { - } \quad \text { Single-hop communication } \\
\text { - } \quad \text { Good solution for nanonode mobility }\end{array}$ & $\begin{array}{l}\text { - Nanorouters (bigger in size) must be fixed over } \\
\text { the medium } \\
\text { Addressing might suppose an inconvenience } \\
\text { when each nanonode has to be identified by a } \\
\text { unique address }\end{array}$ \\
\hline
\end{tabular}

Cluster-based network: a cluster-based hierarchical architecture was first proposed in [1], and it has been taken as a reference for most WNSN topologies. This topology breaks down the WNSN into different tiers, each one at a different level. The lowest level consists of the smallest and less powerful elements, called nanonodes. All nanonodes are grouped into clusters, merely depending on their location. Each cluster is managed by a larger and less resource-constrained device, denoted as nanorouter or nanocontroller, which directly communicates with its cluster, either through single-hop or a multi-hop communication. Finally, nanorouters are usually attached to the so-called gateway which is entrusted with the connection of the WNSN with a (macro) device, for instance, a smartphone or a computer. Under this routing scheme, the assignment of network addresses can be optimized by dividing them into subranges, each one assigned to one cluster, which would act as a subnanonetwork. 


\section{CHAPTER 4. ON THE NATURE OF ENERGY-FEASIBLE WIRELESS 74 NANOSENSOR NETWORKS}

This approach shrinks the number of bits required for addressing, thus reducing the energy employed for data transmission. Furthermore, this hierarchy allows the communication protocol to push the network operation complexity towards the nanorouter, releasing nanonodes from this computing load. This topology supports the design of energy-efficient communication schemes in which each nanonode fits the energy required to send a packet by tuning, in each transmission, different communication parameters, such as power transmission or the number of hops to reach the nanorouter $[2,9,16]$. However, this architecture poses problems when the WNSN is not static, since clusters would change continuously. This variability entails the reconfiguration of the WNSN addressing too frequently, which would result in an excessive waste of energy for nanodevices. Therefore, this topology seems suitable for static WNSN or with limited nanonodes movement (e.g., gathering information about the variation in number and size of cancer cells).

Mesh network: mesh topologies for WNSN are planned to cover a unit of area or volume with a preset number of nanodevices distributed throughout the medium. A good example of this topology is in software-defined metamaterials (SDM), materials whose electromagnetic properties can be reconfigured via software [17]. This technology is enabled by a WNSN composed of identical nanodevices deployed throughout the structure of the metamaterial. The routing in this type of WNSN is based on multi-hop paths to connect two pairs of nanodevices (identified by static or dynamic addresses), which are not usually in coverage range. The principal strength of this network topology lies in the use of a single-type of nanodevice and flood-based schemes to implement nanocommunications [11]. Employing these schemes, when a nanodevice receives a packet, it checks the destination address, which is embedded in the packet. If this address does not match with the nanodevice address under consideration, then it retransmits the packet to its neighbors. However, using this communication mechanism, nanodevices must always be listening to the channel, which entails continuous energy consumption. Alternative routing schemes for mesh WNSN have been proposed [18] with features such as dynamic addresses or synchronization techniques. In comparison with those that are flood-based, these protocols optimize the retransmission process, whereas the processing complexity and memory requirement rise. Therefore, if the energy harvesting rate (i.e., how quickly the energy is gathered) is not high enough, the nanonode consumption under this type of protocols could demand the use of an external energy source. In addition, most of the energy harvested by nanodevices would be spent on retransmission tasks rather than on sending useful information.

Infrastructure-based network: a promising solution for WNSN that are in constant movement (e.g., a WNSN flowing through the bloodstream) is an infrastructure-based topology inspired by conventional mobile networks. In this network, nanorouters are strategically placed at fixed points of the journey. Conversely, nanonodes move through the medium under consideration. Thus, nanonodes are not permanently associated to a nanorouter, but they transmit information to the closest nanorouter in the circuit if and when they are on its coverage range, making this topology a suitable solution for body area nanonetworks (BANN), as proposed in [19]. Finally, nanorouters dispatch all the data collected from nanonodes to the gateway. From the energy point of view, the main advantage of this topology is that the largest energy consumption falls on the nanorouters, whose energy-storing capacity is larger than that in nanonodes. Moreover, since they remain static, an external energy source could easily be implemented to power them. On the other hand, as nanonodes' energy storing is rather limited, they can stay idle while harvesting energy from the environment. When their energy level is high enough, nanonodes wake up to carry out the data transmission to the closest nanorouter by a point-to-point (single-hop) communication. If a nanonode is not within the coverage of any nanorouter, it waits until the signal can be received by one of them. Each nanonode might be identified by a unique address, which is a concern if the number of nodes is huge, due to the large number of bits required for identification. 


\section{CHAPTER 4. ON THE NATURE OF ENERGY-FEASIBLE WIRELESS NANOSENSOR NETWORKS}

\section{Powering Nanodevices}

Owing to the tiny size of nanodevices, manipulating or replacing a depleted battery becomes unfeasible. For this reason, in [6], the authors proposed a built-in piezoelectric generator on each nanodevice to harvest energy from the environment. This type of powering system consists of an array of zinc oxide $(\mathrm{ZnO})$ nanowires, a rectifier circuit and a nanocapacitor, as shown in Figure 2.

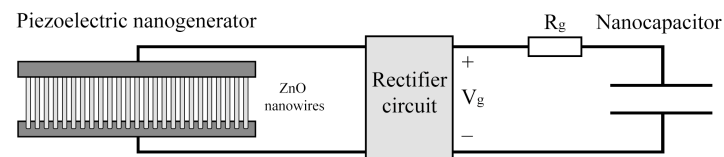

Figure 2. Piezoelectric powering nanosystem diagram.

The operation principle is simple, when the nanowires are bent or compressed, an electric current is generated between the ends of the nanowires, which is used to charge the nanocapacitor. When the nanowires are released, an electric current in the opposite direction is produced allowing the nanocapacitor to be charged once more after rectification. The compress-release cycles of nanowires are induced by an energy source, which could be either natural (e.g., the bloodstream) or artificial (e.g., ultrasound waves) [13]. This solution seems feasible, since both the piezoelectric generator and the capacitor can be miniaturized, ensuring acceptable, but still quite scarce, energy-storing capacity.

In order to illustrate the characteristics of this powering nanosystem, we analyze the results obtained in [6]. The maximum energy stored in the capacitor is $800 \mathrm{pJ}$ and the time required to recharge the capacitor up to $95 \%$ of its total energy capacity is $50 \mathrm{~s}$ or $42 \mathrm{~min}$, depending on the vibration frequency considered. In this case, two sources of energy are suggested, the vents of the air conditioning and the bloodstream, with vibration frequencies of 50 and $1 \mathrm{~Hz}$, respectively. As nanodevices are self-powered and charge and discharge cycles continuously alternate, the frequency of the strains is critical to design a communication scheme, since the charging time varies dramatically depending on the particular energy source. Note that these values are for a nanodevice area of $1000 \mathrm{~mm}^{2}$, which is arguably significant for a nanodevice as the nature of the concept "nanodevice" might be called into question, so these values could be even more restricted.

Even though this solution has been taken as a reference in many works, it was designed for a specific communication scheme. If WNSN conditions change, that is, transmission power rises or the area of the nanodevice needs to be smaller, the design of the powering nanosystem must be adapted. For example, if $100 \mathrm{pJ}$ per pulse are considered, the stored energy in this nanocapacitor is clearly too small for a feasible communication, since the number of pulses that can be transmitted without recharging the capacitor would be 8 at best. Hence, considering a piezoelectric nanogenerator as the powering component for nanodevices, we should highlight three factors that have a direct impact on its performance: (i) area, (ii) technology, and (iii) energy source.

\subsection{Area}

Each $\mathrm{ZnO}$ nanowire generates an electric current when it is bent, so the higher the number of $\mathrm{ZnO}$ nanowires, the higher the charge generated per compress-release cycle. As nanowires have similar diameter (between 50 and $150 \mathrm{~nm}$ ), the total area of the nanogenerator $\left(A_{n g}\right)$ determines the number of $\mathrm{ZnO}$ nanowires that can be integrated. Considering the aforementioned nanogenerator, the charge generated per unit area in each cycle $\left(\Delta Q_{\text {tech }}\right)$ is $6 \mathrm{fC} / \mu \mathrm{m}^{2}$, being the charge generated per cycle $(\Delta Q)$ directly proportional to the area of the piezoelectric nanogenerator: $\Delta Q=\Delta$ Qtech $A_{n g}$. To evaluate how $A_{n g}$ affects the energy-harvesting rate $(\lambda)$, we derive its expression by modelling the generator as a voltage source $\left(V_{g}\right)$ along with a resistor $\left(R_{g}\right)$ in series, following the methodology proposed in [6] Thus, the resistor value is $R_{g}=V_{g} / I_{g}$, where $I_{g}$ is the average generator current per cycle. $I_{g}$ is defined 


\section{CHAPTER 4. ON THE NATURE OF ENERGY-FEASIBLE WIRELESS}

as $I_{g}=\Delta Q f=\Delta Q$ tech $A_{n g} f$, where $f$ is the strain frequency. Using this model, the expression that characterizes the charge stored in a capacitor as a function of the time is as follows:

$$
Q(t)=C_{n c} V_{g}\left(1-e^{\left(\frac{-t}{R_{g} C_{n c}}\right)}\right)=C_{n c} V_{g}\left(1-e^{\left(\frac{-t \Delta Q_{\text {tech }} A_{n g} f}{V g C_{n c}}\right)}\right)
$$

where $C_{n c}$ stands for the nanocapacitor capacitance. The energy stored in a nanocapacitor $\left(E_{n c}\right)$ is stated as a function of the charge $(Q)$ :

$$
E_{n c}=\frac{Q^{2}}{2 C_{n c}}
$$

Combining expressions (1) and (2), the energy stored in a nanocapacitor as a function of time, assuming that the capacitor is initially empty, is as follows:

$$
E_{n c}(t)=\frac{C_{n c} V_{g}^{2}}{2}\left(1-e^{\left(\frac{-t \Delta Q_{\text {tech }} A_{n g} f}{V_{g} C_{n c}}\right)}\right)^{2}
$$

The energy harvesting rate $(\lambda)$ is defined as the variation of energy stored in the nanocapacitor as a function of time, so we obtain its expression by means of the time derivative of $E_{n c}(t)$ :

$$
\lambda(t)=\frac{d E_{n c}(t)}{d t}=V_{g} \Delta Q_{t e c h} A_{n g} f\left(1-e^{\left(\frac{-t \Delta Q_{\text {tech }} A_{n g} f}{V g C h c}\right)}\right) e^{\left(\frac{-t \Delta Q_{t e c h} A n g f}{V_{g} C_{n c}}\right)}
$$

Figure 3 shows $\lambda$ as a function of time for different nanogenerator areas, with $\Delta Q_{t e c h}, f, V_{g}$, and $C_{n c}$ equal to $6 \mathrm{fC} / \mu \mathrm{m}^{2}, 1 \mathrm{~Hz}, 0.42 \mathrm{~V}$, and $9 \mathrm{nF}$, respectively. Note that these parameters have been fixed in order to properly appreciate the impact of $A_{n g}$. Even though these values could be arbitrarily set (within reasonable bounds), we prefer extracting them from an already published work to be as rigorous as possible [6]. As can be seen, when $A_{g}$ becomes larger, the peak of $\lambda$ increases, leading to a quicker nanocapacitor charge. After the maximum, the value of $\lambda$ decreases, tending to zero, indicating that the nanocapacitor is reaching its maximum storing capacity.

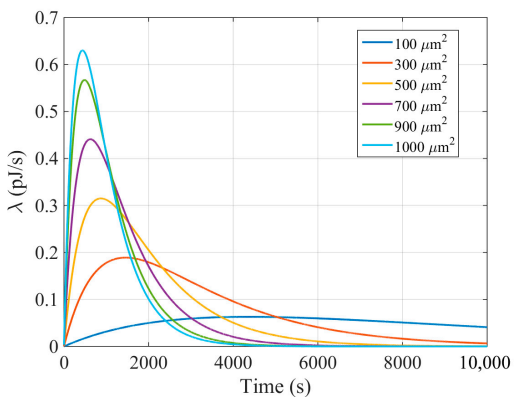

Figure 3. Energy-harvesting rate as a function of time for different nanogenerator areas.

In turn, the area of the nanocapacitor $\left(A_{n c}\right)$ influences its capacitance $\left(C_{n c}\right)$, which is related to the maximum stored energy $\left(E_{\max }\right)$ by the expression:

$$
E_{\max }=\frac{V_{g}^{2} C_{n c}}{2}
$$

where $V_{g}$ is the voltage generator. As $C_{n c}=C_{t e c h} A_{n c}$, where $C_{t e c h}$ is the capacitance per unit of area, for a fixed voltage generator, the maximum stored energy is directly proportional to the capacitance 


\section{CHAPTER 4. ON THE NATURE OF ENERGY-FEASIBLE WIRELESS NANOSENSOR NETWORKS}

and, consequently, to the nanocapacitor area. However, as $E_{\max }$ increases, the time to charge the nanocapacitor becomes longer, so a trade-off emerges between these two parameters. Consequently, this issue directly impacts on the achievable bitrate per nanodevice $(R)$, since nanodevices must remain idle during the charging time $\left(t_{\text {charge }}\right)$.

To illustrate this point, we obtain the equation to calculate the time required to accumulate a given amount of energy by isolating the variable $t$ from expression (3):

$$
t\left(E_{c}\right)=-\frac{C_{t e c h} A_{n c} V_{g}}{\Delta Q_{t e c h} A_{n g} f} \ln \left(1-\sqrt{\frac{2 E_{c}}{V_{g}^{2} C_{t e c h} A_{n c}}}\right)
$$

Note that $C_{n c}$ has been expressed as a function of $A_{n c}$. If $E_{c}=E_{\text {total }}=E_{\text {pulse }} n_{\text {pulse }}+P_{\text {dev }} t_{\text {on }}$, then this time corresponds to $t_{\text {charge }}$ :

$$
t_{\text {charge }}=-\frac{C_{\text {tech }} A_{n c} V_{g}}{\Delta Q_{\text {tech }} A_{n g} f} \ln \left(1-\sqrt{\frac{2\left(E_{\text {pulse }} n_{\text {pulse }}+P_{\text {dev }} t_{\text {on }}\right)}{V_{g}^{2} C_{\text {tech }} A_{n c}}}\right)
$$

For the sake of simplicity, since the objective is to emphasize the impact of $A_{n c}$ on $t_{\text {charge }}$ and thus the achievable bitrate per nanodevice, we assume that the capacitor is empty at the beginning of the recharge period and all the energy is consumed in the transmission. We note that due to the non-linear charging behavior of a capacitor, an optimal range of charge could be found preventing the capacitor from being fully depleted or completely charged, but the influence of $A_{n c}$ remains the same. Thus, as the communication starts after a period of time equal to $t_{\text {charge }} R$ can be expressed as:

$$
R=\frac{n_{\text {bits }}}{t_{\text {charge }}}=-\frac{n_{\text {bits }} \Delta Q_{\text {tech }} A_{n g} f}{C_{\text {tech }} A_{n c} V_{g} \ln \left(1-\sqrt{\frac{2\left(E_{\text {pulse }} n_{\text {bits }} p_{\text {pulse }}+P_{\text {dev }} t_{\text {on }}\right)}{V_{g}^{2} C_{\text {tech }} A_{n c}}}\right)},
$$

where $n_{\text {bits }}$ stands for the number of bits transmitted with a single charge and $n_{\text {pulse }}$ is expressed as a function of the probability of sending a pulse $\left(p_{\text {pulse }}\right): n_{\text {pulse }}=n_{\text {bits }} p_{\text {pulse }}$.

Figure 4 shows $R$ as a function of $A_{n c}$ for the example given in Section 2. The values employed for $E_{\text {pulse }}, n_{\text {bits }}, p_{\text {pulse }}, P_{\text {dev }}, t_{\text {on }}, V_{g}, C_{\text {tech }}, Q_{\text {tech }}, A_{n g}$, and $f$ are $1 \mathrm{pJ}, 169 \mathrm{bits}, 0.5,240 \mathrm{nW}, 1 \mathrm{~ms}, 0.42 \mathrm{~V}$, $9 \mathrm{pF} / \mu \mathrm{m}^{2}, 6 \mathrm{fC} / \mu \mathrm{m}^{2}, 1000 \mu \mathrm{m}^{2}$, and $1 \mathrm{~Hz}$ respectively. The value of each one could be arbitrarily set but, maintaining the rigor of the analysis, has been taken from published works $[6,13]$ already validated by the research community. As can be observed, for a specific application the bitrate achieved per nanodevice can be optimized by selecting the area of the nanocapacitor properly. This is due to the fact that for a given amount of energy required there is an optimal capacity that minimizes the charging time. If the capacitance becomes higher, the maximum energy stored increases, but the period of time to store this determined amount of energy $\left(t_{\text {charge }}\right)$ is higher than in a capacitor with a lower capacitance. This increase of time hinders the bitrate achieved by nanodevices. To the best of our knowledge, this issue has never been considered before in the related literature when designing either a nanonetwork or a nanocommunication protocol. Thus, considering a particular technology with its corresponding $C_{t e c h}$, there is an $A_{n c}$ for which the bitrate is maximum. In the example above, to transmit 169 bits with one single charge, the maximum bitrate is achieved for a nanocapacitor area equal to $268 \mu \mathrm{m}^{2}$

Therefore, the areas of both components of the powering nanosystem are critical to further design nanocommunications, since it will influence $\lambda, E_{\max }$ and $R$. 


\section{CHAPTER 4. ON THE NATURE OF ENERGY-FEASIBLE WIRELESS}

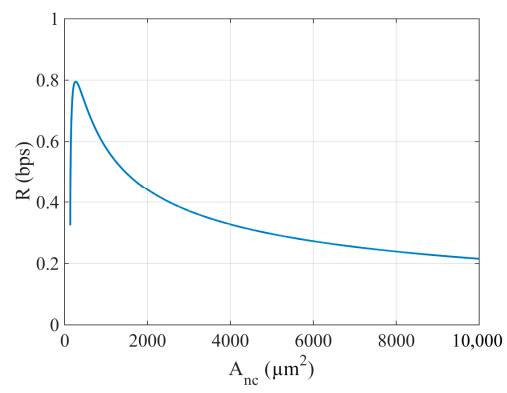

Figure 4. Bitrate per nanodevice as a function of the nanocapacitor area.

\subsection{Technology}

A nanogenerator based on $\mathrm{ZnO}$ nanowires is a promising technology for nanodevices, but its limited energy harvesting becomes evident when its area shrinks. Alternative solutions that improve the energy-harvesting efficiency should gain increasing importance to shorten the charging time of nanodevices, which is of special relevance in scenarios with weak or scarce mechanical forces. Furthermore, as mentioned before, the maximum storable energy in a nanocapacitor depends on its capacitance, which is ultimately determined by the nanocapacitor technology. For instance, Jornet and Akyildiz proposed a nanocapacitor based on onion-like carbon electrodes, which could reach a capacitance of $9 \mathrm{nF}$ for an area of $1000 \mu \mathrm{m}^{2}$, that is, $C_{t e c h}$ equal to $9 \mathrm{pF} / \mu \mathrm{m}^{2}$ [6]. Instead, in [20], a so-called supercapacitor is designed and fabricated with a $C_{t e c h}$ of $400 \mathrm{pF} / \mu \mathrm{m}^{2}, 44$ times higher than the former. This increase in capacitance means 44 times more energy-storing capacity for the same area. This is merely an example of the potential of novel capacitor technologies, which should be reviewed for future research in order to adapt communication techniques for WNSN to the energy-storing capacity available.

\subsection{Energy Source}

Two energy sources have been proposed for WNSN so far [6,21], both harnessing the piezoelectric nature of the nanogenerator, converting mechanical energy into electricity.

Energy harvesting from the environment: this solution is envisaged for WNSN deployed in dynamic environments, where the mechanical forces induced by the medium generate energy enough to power nanodevices. However, to the best of our knowledge, no energy model for nanodevices has studied how the energy-harvesting rate varies for different scenarios in terms of strain magnitude and frequency. In this respect, more effort is required to acquire realistic information.

Wireless power transfer (WPT) with ultrasound: using WPT, the piezoelectric nanogenerator vibrates at the ultrasound frequency, generating compress-release cycles regardless of the environment. This external power supply ensures that the energy-harvesting rate is constant and irrespective of the specific environmental conditions.

In order to compare the energy harvested for both energy sources, Figure 5 illustrates the percentage of stored energy as a function of time for different strain frequencies and WPT, obtained from the ratio between expressions (3) and (5). Values of $\Delta Q_{t e c h}, A_{n g}, V_{g}$, and $C_{n c}$ are equal to $6 \mathrm{fC} / \mu \mathrm{m}^{2}, 100 \mu \mathrm{m}^{2}$, $0.42 \mathrm{~V}$, and $9 \mathrm{nF}$, respectively. As can be seen, when applying WPT, the energy harvested per unit of time is up to three orders of magnitude higher than the energy gathered from environments with low-frequency strains such as the bloodstream, whose strain frequency fluctuates between $1 \mathrm{and} 3 \mathrm{~Hz}$. 


\section{CHAPTER 4. ON THE NATURE OF ENERGY-FEASIBLE WIRELESS} NANOSENSOR NETWORKS

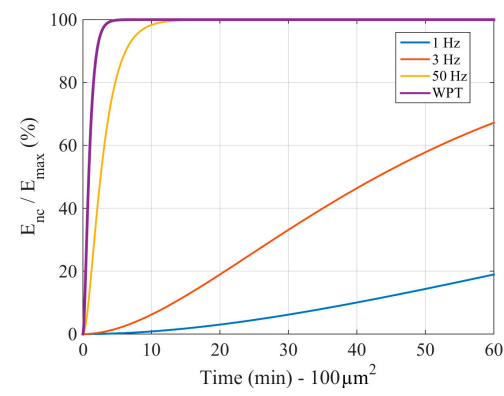

Figure 5. Percentage of energy stored as a function of time for different energy source frequencies.

\section{Energy Feasibility of a WNSN}

Once all the factors governing the energy management have been analyzed, in this section we study the feasibility of a WNSN from the energetic point of view, independent of the network topology employed. To this effect, we first calculate the minimum nanocapacitor area and its charging time, considering energy per pulse and number of pulses radiated as variables. Then, we derive the same area figure but this time characterizing the propagation channel by path loss and transmission distance.

Let us consider that the total energy consumption must be equal to or lower than the $95 \%$ of the total storing capacity of the nanocapacitor, defined by expression (5). This is because the time required to charge a capacitor up to its theoretical $E_{\max }$ tends to infinite, so the upper limit of charge is set to $95 \%$. Therefore, $E_{\text {pulse }} n_{\text {pulse }}+P_{\text {dev }} t_{\text {on }} \leq 0.95 V_{g}{ }^{2} C_{n c} / 2$.

Joining both expressions, the minimum area of the nanocapacitor $\left(A_{\min }\right)$ is obtained as a function of $E_{\text {pulse }}$ and $n_{\text {pulse }}$ :

$$
A_{\min }=\frac{2\left(E_{\text {pulse }} n_{\text {pulse }}+P_{\text {dev }} t_{o n}\right)}{0.95 C_{t e c h} V_{g}^{2}}
$$

In Figure $6, A_{\min }$ is depicted as a function of $E_{\text {pulse }}$ and $n_{\text {pulse, }}$, while the remaining factors of the expression keep constant. Concretely, values considered for $P_{d e v}, t_{o n}, C_{t e c h}$, and $V_{g}$ are extracted from [6,13]: $240 \mathrm{nW}, 1 \mathrm{~ms}, 9 \mathrm{pF} / \mu^{2}$, and $0.42 \mathrm{~V}$, respectively. As can be observed, the capacitor area required to properly power a nanodevice becomes excessive when the energy per pulse is close to $100 \mathrm{pJ}$; a usual value in nanocommunication papers. Only if a few pulses are radiated does the capacitor area remain small enough to be considered a nanodevice.

We now discuss the viability of the powering nanosystem described in Section 3. Based on expression (7), Figure 7 shows $t_{\text {charge }}$ as a function of the energy per pulse and the number of pulses. Values employed for $P_{d e v}, t_{o n}, C_{n c}, V_{g}, \Delta Q$, and $f$ are $240 \mathrm{nW}, 1 \mathrm{~ms}, 9 \mathrm{nF}, 0.42 \mathrm{~V}, 6 \mathrm{pC}$, and $1 \mathrm{~Hz}$ extracted from $[6,13]$. As can be seen, there is a delimited curve for which $t_{\text {charge }}$ tends to infinity, which means that the nanocapacitor is reaching its $E_{\max }$. Beyond this curve, $t_{\text {charge }}$ is equal to 0 , determining the unfeasible energetic zone (in grey), that is, the powering nanosystem is not able to supply enough energy to the nanodevice. In Figure 7 (right), we clearly observe the values of $E_{\text {pulse }}$ and $n_{\text {pulse }}$, achievable with one single charge (that corresponds to the $95 \%$ of the theoretical maximum energy stored in the capacitor, as mentioned above) for which the powering nanosystem is feasible. It is noteworthy that the number of pulses when $E_{\text {pulse }}$ is higher than $10 \mathrm{pJ}$ becomes excessively low $(<50$ pulses $)$ to perform a consistent communication scheme. 


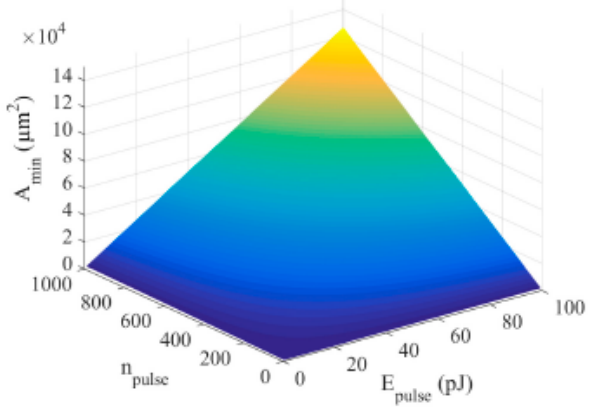

Figure 6. Minimum capacitor area as a function of pulse energy and number of pulses.

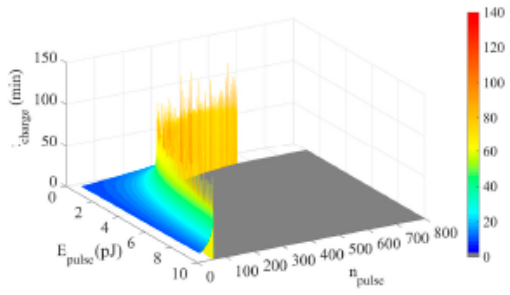

(a)

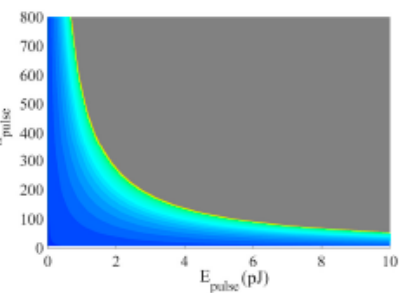

(b)

Figure 7. Charging time for different pulse energy and number of pulses (a). Same graph from top view (b).

Later, we evaluate the feasibility of this powering nanosystem for different environments and transmission distances. To accomplish the communication properly, the transmission power $\left(P_{t x}\right)$ must satisfy $10 \log _{10}\left(P_{t x}\right)-L_{\text {path }} d \geq S$, where $L_{\text {path }}$ is the path loss per unit of distance (in $\mathrm{dB} / \mathrm{mm}$ ) per unit of distance, $d$ is the distance (in $\mathrm{mm}$ ), and $S$ is the receiver sensitivity (in dBW). $P_{t x}$ is set as the relation between the energy per pulse $\left(E_{\text {pulse }}\right)$ and its duration $\left(t_{\text {pulse }}\right): P_{t x}=E_{p u l s e} / t_{\text {pulse }}$. Considering both expressions, the minimum $E_{\text {pulse }}$ to fulfill the communication is:

$$
E_{\text {pulse }}=t_{\text {pulse }} 10^{\frac{s+L_{\text {path }} d}{10}}
$$

Substituting $E_{\text {pulse }}$ in (9), we obtain the following formula:

$$
A_{\min }=\frac{2\left(t_{\text {pulse }} 10^{\frac{s+L_{\text {puth }} d}{10}} n_{\text {pulse }}+P_{\text {dev }} t_{\text {on }}\right)}{0.95 C_{\text {tech }} V_{g}^{2}}
$$

Considering $S=-130 \mathrm{dBW}(-100 \mathrm{dBm})$ and $n_{\text {pulse }}=85$ (i.e., enough to accomplish the blood cholesterol application proposed in a previous section), Figure 8 shows the minimum nanogenerator area $\left(A_{\min }\right)$ in terms of path loss per unit of distance $\left(L_{\text {path }}\right)$ and transmission distance $(d)$. Values considered for $t_{\text {pulser }}, P_{\text {dew }}, t_{\text {on }}, C_{\text {tech }}$, and $V_{g}$ are $100 \mathrm{fs}, 240,1 \mathrm{~ms}, 9 \mathrm{pF} / \mu \mathrm{m}^{2}$, and $0.42 \mathrm{~V}$ respectively, extracted from $[6,13]$. 


\section{CHAPTER 4. ON THE NATURE OF ENERGY-FEASIBLE WIRELESS NANOSENSOR NETWORKS}

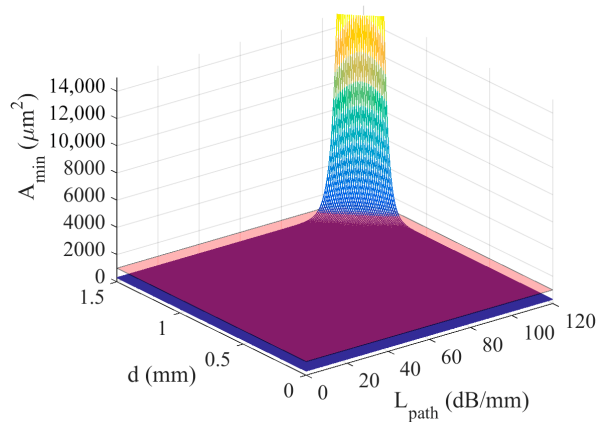

Figure 8. Minimum capacitor area as a function of path loss and transmission distance.

Results reveal that there is a delimited zone (illustrated by a red plane) for which the nanogenerator under study is feasible from the energetic point of view. Note that this solution is unfeasible for transmission distances higher than $1.5 \mathrm{~mm}$ in environments with path loss greater than $100 \mathrm{~dB} / \mathrm{mm}$. Therefore, WNSN must be designed bearing in mind this hard energy restriction, since the nanocapacitor area required when high path losses become excessively high falls outside the nanodevice concept. Therefore, nanocommunications should stay within the feasibility area (in terms of path loss and distance) to be realistic and viable.

\section{Conclusions}

This paper analyzes energy management as a critical issue to be considered in future WNSN designs. We first discuss the main factors influencing the energy consumption in WNSN, highlighting the importance of the energy required for data transmission. Then, we review the most relevant parameters impacting on the functioning of a powering nanosystem, examining the most accepted solution for nanodevices, and evaluating its performance under different conditions After a mathematical analysis, results reveal these main conclusions: (i) many already published works employed high transmission powers (even peaks of kilowatts) inadvisable for a nanogenerator with the technology proposed in [6]; (ii) the WNSN, bitrate can be optimized for a specific application by designing nanodevices with a determined nanocapacitor area; (iii) the energy harvested per unit of time by WPT is up to three orders of magnitude higher than the energy gathered from the environment; (iv) charging time remains acceptable if and when the energy required is not excessive (an unfeasible energetic zone for WNSN is defined); and (v) the solution in [6] becomes inoperative for transmission distances over $1.5 \mathrm{~mm}$ in high-lossy environments (exceeding $100 \mathrm{~dB} / \mathrm{mm}$ ), although this is the scenario usually found when applying WNSN to medicine. Regarding network topologies, more research is needed to numerically estimate and compare their impact on energy consumption. This requires further study and is, therefore, the subject of a future work.

Author Contributions: S.C.-C. developed the mathematical analysis and figures and looked into the related literature. A.-J.G.-S. and J.G.-H. supervised the analysis and evaluated the results. All authors conceived the idea and participated in the elaboration of the manuscript.

Acknowledgments: This work has been supported by the project AIM, ref. TEC2016-76465-C2-1-R (AEI/FEDER, UE). Sebastian Canovas-Carrasco also thanks the Spanish MECD for an FPU (ref. FPU16/03530) pre-doctoral fellowship.

Conflicts of Interest: The authors declare no conflict of interest. 


\section{CHAPTER 4. ON THE NATURE OF ENERGY-FEASIBLE WIRELESS}

\section{References}

1. Akyildiz, I.; Jornet, J. The Internet of nano-things. IEEE Wirel. Commun. 2010, 17, 58-63. [CrossRef]

2. Pierobon, M.; Jornet, J.M.; Akkari, N.; Almasri, S.; Akyildiz, I.F. A routing framework for energy harvesting wireless nanosensor networks in the Terahertz Band. Wirel. Netw. 2014, 20, 1169-1183. [CrossRef]

3. Jornet, J M.; Akyildiz, I.F. Femtosecond-Long Pulse-Based Modulation for Terahertz Band Communication in Nanonetworks. IEEE Trans. Commun. 2014, 62, 1742-1754. [CrossRef]

4. Jornet, J.M.; Capdevila Pujol, J.; Solé Pareta, J. PHLAME: A Physical Layer Aware MAC protocol for Electromagnetic nanonetworks in the Terahertz Band. Nano Commun. Netw. 2012, 3, 74-81. [CrossRef]

5. Wang, P.; Jornet, J M.; Abbas Malik, M.G.; Akkari, N.; Akyildiz, I.F. Energy and spectrum-aware MAC protocol for perpetual wireless nanosensor networks in the Terahertz Band. Ad Hoc Netw. 2013, 11, 2541-2555. [CrossRef]

6. Jornet, J.M.; Akyildiz, I.F. Joint Energy Harvesting and Communication Analysis for Perpetual Wireless Nanosensor Networks in the Terahertz Band. IEEE Trans. Nanotechnol. 2012, 11, 570-580. [CrossRef]

7. Piro, G.; Bia, P.; Boggia, G.; Caratelli, D.; Grieco, L.A.; Mescia, L. Terahertz electromagnetic field propagation in human tissues: A study on communication capabilities. Nano Commun. Netw. 2016, 10, 51-59. [CrossRef]

8. Jornet, J.M.; Akyildiz, I.F. Channel modeling and capacity analysis for electromagnetic wireless nanonetwork in the terahertz band. IEEE Trans. Wirel. Commun. 2011, 10, 3211-3221. [CrossRef]

9. Afsana, F.; Asif-Ur-Rahman, M.; Ahmed, M.R.; Mahmud, M.; Kaiser, M.S. An Energy Conserving Routing Scheme for Wireless Body Sensor Nanonetwork Communication. IEEE Access 2018, 6, 9186-9200. [CrossRef]

10. Piro, G.; Boggia, G.; Grieco, L.A. On the design of an energy-harvesting protocol stack for Body Area Nano-NETworks. Nano Commun. Netw. 2015, 6, 74-84. [CrossRef]

11. Tsioliaridou, A.; Liaskos, C.; Ioannidis, S.; Pitsillides, A. Lightweight, self-tuning data dissemination for dense nanonetworks. Nano Commun. Netw. 2015, 8, 2-15. [CrossRef]

12. Abbasi, Q.H.; Nasir, A.A.; Yang, K.; Qaraqe, K.A.; Alomainy, A. Cooperative In-Vivo Nano-Network Communication at Terahertz Frequencies. IEEE Access 2017, 5, 8642-8647. [CrossRef]

13. Canovas-Carrasco, S.; Garcia-Sanchez, A.-J.; Garcia-Sanchez, F.; Garcia-Haro, J. Conceptual Design of a Nano-Networking Device. Sensors 2016, 16, 2104. [CrossRef] [PubMed]

14. Jornet, J.M. Low-weight error-prevention codes for electromagnetic nanonetworks in the Terahertz Band Nano Commun. Netw. 2014, 5, 35-44. [CrossRef]

15. Yang, K.; Pellegrini, A.; Munoz, M.O.; Brizzi, A.; Alomainy, A.; Hao, Y. Numerical Analysis and Characterization of $\mathrm{THz}$ Propagation Channel for Body-Centric Nano-Communications. IEEE Trans. Terahertz Sci. Technol. 2015, 5, 419-426. [CrossRef]

16. Lee, S.J.; Jung, C.; Choi, K.; Kim, S. Design of Wireless Nanosensor Networks for Intrabody Application. Int. J. Distrib. Sens. Netw. 2015, 11, 176761. [CrossRef]

17. Abadal, S.; Liaskos, C.; Tsioliaridou, A.; Ioannidis, S.; Pitsillides, A.; Sole-Pareta, J.; Alarcon, E.; Cabellos-Aparicio, A. Computing and Communications for the Software-Defined Metamaterial Paradigm A Context Analysis. IEEE Access 2017, 5. [CrossRef]

18. Tsioliaridou, A.; Liaskos, C.; Dedu, E.; Ioannidis, S. Packet routing in 3D nanonetworks: A lightweight, linear-path scheme. Nano Commun. Netw. 2016, 12, 63-71. [CrossRef]

19. Canovas-Carrasco, S.; Garcia-Sanchez, A.-J.; Garcia-haro, J. A nanoscale communication network scheme and energy model for a human hand scenario. Nano Commun. Netw. 2018, 15, 17-27. [CrossRef]

20. El-Kady, M.F.; Ihns, M.; Li, M.; Hwang, J.Y.; Mousavi, M.F.; Chaney, L.; Lech, A.T.; Kaner, R.B. Engineering three-dimensional hybrid supercapacitors and microsupercapacitors for high-performance integrated energy storage. Proc. Natl. Acad. Sci. USA 2015, 112, 4233-4238. [CrossRef] [PubMed]

21. Donohoe, M.; Balasubramaniam, S.; Jennings, B.; Jornet, J M. Powering In-Body Nanosensors with Ultrasounds. IEEE Trans. Nanotechnol. 2016, 15, 151-154. [CrossRef] 
Chapter 5

The IEEE 1906.1 standard:

Some guidelines for

strengthening future

normalization in electromagnetic nanocommunications 
CHAPTER 5. THE IEEE 1906.1 STANDARD: SOME GUIDELINES FOR 84

FUTURE NORMALIZATION IN EM NANOCOM.

\title{
THE IEEE 1906.1 STANDARD: SOME GUIDELINES FOR STRENGTHENING FUTURE NORMALIZATION IN ELECTROMAGNETIC NANOCOMMUNICATIONS
}

\author{
Sebastian Canovas-Carrasco, Antonio-Javier Garcia-Sanchez, Joan Garcia-Haro \\ Department of Information and Communication Technologies, Technical University of Cartagena, \\ Cartagena, Spain. \\ Emails: sebas.canovas@upct.es, antoniojavier.garcia@upct.es, joang.haro@upct.es
}

\begin{abstract}
Nanoscale communications is a new paradigm encompassing all concerns related to the exchange of information among devices at the nanometer scale. A network infrastructure consisting of a huge quantity of nano-devices is envisaged to ensure robust, reliable and coordinated data transmission. This will enable a plethora of forthcoming applications and services in many differen research fields, such as personalized medicine, synthetic biology, environmental science, or industry, which will lead to outstanding and unprecedented advances. The IEEE 1906.1 standard provides a conceptual and general framework to set the starting point for future developments in nanoscale communication networks. This paper reviews the latest IEEE 1906.1 recommendations, observing their main features when applied to the electromagnetic (EM) nanocommunication area. We contribute by identifying the standard achievements and indicating future guidelines for the development of the upcoming standardization of EM nanoscale communications.
\end{abstract}

Keywords - Nanoscale communication networks, nanodevices, EM nanocommunications, terahertz communications, IEEE standards

\section{INTRODUCTION}

In the emerging Internet of Things (IoT), objects are expected to be able to sense and capture the physical variables of their surroundings (e $g$ temperature, humidity, pressure, etc ) as well as to process the acquired information and communicate it wirelessly to any other object/node in their network These enhanced objects integrate small sensing/computing/communicating devices in a varied range of sizes, including the nanoscale Moreover, devices in the IoT constitute a network infrastructure connecting both physical and virtual worlds by means of all sorts of innovative applications and services, some of which are currently unimaginable Nowadays, the IoT relies on the well-known Wireless Sensor Networks (WSN), in which

This work has been supported by the project AIM, ref TEC2016-76465-C2-1-R (AEI/FEDER, UE) Sebastian Canovas-Carrasco also thanks the Spanish MECD for an FPU (ref FPU16/03530) pre-doctoral fellowship numerous devices with limited resources are interconnected, in order to provide feasible solutions in multiple heterogeneous fields, such as agriculture, industry, and smart cities, among others Keeping in mind the way WSN operate and due to incessant technological advances, novel devices with progressively smaller dimensions are being developed to ease their integration into the environment However, as they become smaller, many concerns, such as available energy, transmission range or data processing capacity, are far more restricted than in traditional WSN Thus, when the scale of these tiny devices decreases to nanometers, a new paradigm arises; nanoscale communications between nanomachines, and between nanomachines and more conventional devices in the network

These nanodevices have become a topic of increasing interest to the scientific community, since they would be able to gather physical parameters at the nanoscale with outstanding accuracy This capacity would allow the monitoring of scenarios not explored to date, enabling a myriad of potential applications in fields as varied as biomedicine, synthetic biology, environmental science or industry, among many others Indeed, one of the most promising applications of these nanodevices is aimed at improving medicine, because diverse medical tests, such as blood pressure, virus detection or oxygen levels in blood could be collected in vivo and directly transmitted to medical personnel (e g information about the variation in number and size of cancer cells will be received by the oncologist)

Several works have dealt with how nanodevices should communicate with each other This is becoming a critical issue, since the extremely limited resources of nanodevices require them to work cooperatively to perform a useful function Two main alternatives for communicating at the nanoscale have been envisaged so far, electromagnetic (EM) and molecular communication

EM communication is based on the use of electromagnetic waves to transmit a message between two nanodevices Advancements in carbon electronics, mainly those devices made of graphene and carbon nanotubes (CNT), are playing a key role in the development of a new generation of electronic nanocomponents, such as nanoantennas or 2471-2825/18/\$25 00 C 2018 IEEE DOI: 10 1109/MCOMSTD 20181700082 


\section{CHAPTER 5. THE IEEE 1906.1 STANDARD: SOME GUIDELINES FOR}

FUTURE NORMALIZATION IN EM NANOCOM.

nanotransceivers [1], [2] These novel radiocommunication nanocomponents possess unbeatable properties, which allow the radiation of EM waves at $\mathrm{THz}$ frequencies with antennas of just a few micrometers in length, $i$ e two orders of magnitude smaller than their metallic counterparts Even so, this radiation frequency exhibits high propagation losse which, together with the energy scarcity in nanodevices, require a complete nanoscale communication network design, also known as a nanonetwork On the other hand, molecular communication is defined as the transmission an reception of information encoded in organic molecules [3] Molecular transceivers are designed to facilitate thei integration into nanodevices, due to their extremely small size and limited domain of operation These transceiver can react when receiving certain molecules and release others (as a response to stimulation or after executing some process)

In this context, the IEEE 19061 standard emerges as the first approach to fill an important research gap and to normalize diverse aspects related to communications at the nanoscale, considering both EM and molecular communications [4] Under this general premise, this standard first defines the concept of a nanoscale communication network itself, to later propose a conceptual framework for developing communications Studies using the guidelines of this standard would contribute to the implementation of a viable protocol stack based on th components and procedures specified Thus, the results obtained for each design could be shared and compared from a common set of performance metrics defined by the standard This paper reviews the IEEE 19061 standard, focusing on EM communications; an area in which remarkable technological advances are leading to the first realistic approaches at the nanoscale In particular, we analyze the standard definition, its pros and cons, an describe the framework offered along with its components Always referring to the standard approach, we identify open issues and challenges that should be covered in future versions (aiming at EM nanocommunications) to fulfill the purpose for which it was conceived As the standard is still in an early stage, we do not provide specific solutions for specific problems, since each solution requires a research process before standardization, which falls out of the scope of this paper Thus, we lay the foundation and point out the way to proceed in each one of the six different topics related to EM nanocommunications we address

\section{OVERVIEW OF THE IEEE 1906.1 STANDARD} FOR EM NANOSCALE COMMUNICATIONS As interdisciplinary research groups are becoming more an more involved in the development of nanoscale communications, the lack of a clear common scope has been made evident, leading to isolated developments an unrelated islands of knowledge In this sense, the different cases of study proposed in the open scientific literature have been thought about and evaluated under very specific conditions, which may differ for each work This has a negative impact on the exchange of information in nanonetworks, since their performance depends on the particular working conditions and capabilities of nanodevices Thus, the IEEE 19061 standard [5] is aimed at providing a common framework, in order to join forces and promote future advances in nanoscale communications In addition, another significant contribution of this standard is to allow sufficient precision for the development of interoperable and reusable components In order to provide insight into this standard, we examine the following three aspects of its structure: (i) definitions, (ii) framework, and (iii) metrics

\subsection{DEFINITIONS}

The first part of the standard offers a detailed definition of "nanoscale communication network", which should pave the way for future studies in this emerging research field This definition intends to strictly establish the scope of this concept but to keep it general enough to cover both molecular and EM communications The range chosen to delimit the nanoscale is quite narrow, from $1 \mathrm{~nm}$ to $100 \mathrm{~nm}$ The lower limit has been selected to exclude the use of single atoms as nanoscale systems In contrast, the upper limit is the size at which material properties change substantially from the macroscale This limit could cause controversy, since most of the scientific papers related to EM nanoscale communications consider nanodevices at larger scales Nevertheless, the sentence "at or with the nanoscale" contained in the standard definition leaves the door open to different considerations, in particular, those concerning the size of the nanoscale object under study Therefore, those nanodevices including a communication element (transmitter, receiver, medium, message, and message carrier) at the nanoscale would be covered by the standard

\subsection{FRAMEWORK}

The second block defined by the standard provides a general, conceptual, small-scale framework consisting of an appropriate number of components with well-defined functions and interoperability among them The framework offers the organization and structure required to implement procedures and models To this end, a set of interconnecting components is introduced, namely: (i) message carrier, (ii) motion, (iii) field, (iv) perturbation, and (v) specificity

The message carrier is described as the physical entity which transports the message across the medium In particular, in EM nanocommunications, message carrier would indicate the EM wave The perturbation componen refers to the mechanism required to accommodate the message carrier to the medium in order to transmit the signal that contains the message Referring to EM communication, this component would cover parameters such as the central frequency of the EM wave (in order to be considered nanoscale communication, in the $\mathrm{THz}$ band), bandwidth (usually from $055 \mathrm{THz}$ to $155 \mathrm{THz}$ ), transmission power, pulse features or type of modulation The motion component represents the physical phenomenon that enables the message carrier to move (in EM, the wave propagation and phase velocity) It is created based on the 
Table 1 Example of the equivalence between EM nanoscale network components and the IEEE 19061 framework

\begin{tabular}{|l|l|}
\hline $\begin{array}{l}\text { IEEE 1906.1 } \\
\text { component }\end{array}$ & Implemented component \\
\hline Transmitter & CNT-based nanoantenna \\
\hline Receiver & CNT-based nanoantenna \\
\hline Message & Sodium concentration \\
\hline Medium & Air \\
\hline Message carrier & Electromagnetic (EM) wave \\
\hline Component $<100 \mathrm{~nm}$ & $\begin{array}{l}\text { Sensor, message carrier } \\
\text { (THz frequency wave) }\end{array}$ \\
\hline Non-standard physics & Impact of scale on resonance \\
\hline Motion & Radiation and waveguide \\
\hline Field & Intensity/directional antenna \\
\hline Perturbation & RF modulation \\
\hline Specificity & $\begin{array}{l}\text { Receptor sensitivity/antenna } \\
\text { aperture }\end{array}$ \\
\hline
\end{tabular}

propagation model in the scenario under consideration This component may be randomly propagated through the medium, which would hamper the propagation of the wave Therefore, the field component organizes and guides the movement of the motion component Concerning the EM nanocommunication system, this would correspond to the antenna Finally, specificity refers to the reception of the message carrier by a specific receiver (receive sensitivity/antenna aperture) It verifies that all the parameters stored within the received message carrier ar the same as those contained in the receiver perturbation component Table 1 (extracted from [5]) shows all the framework components with their corresponding elements in a EM nanoscale communication example

This framework is compared to the Open System Interconnection (OSI) model, in order to place the five aforementioned components in the communication protoco stack of reference, as specified in Table 2 (extracted from [5]) Due to their close relation to physical aspects, the nanoscale framework components are situated in the lower layers of the OSI stack, even breaching the separation between them

\subsection{METRICS}

The third section of the standard addresses the definition of common metrics to give information about the interoperability among system components, together with the computation and comparison of performance in a nanoscale communication network Evaluating networks by using these metrics, researchers can objectively compare the grade of improvement or deterioration that different nanoscale network designs experience

Moreover, in order to provide a common environment for development, the standard proposes a discrete-event and open source network simulator implemented in the Network
Table 2 OSI to nanoscale communication network mapping

\begin{tabular}{|c|c|c|c|}
\hline $\begin{array}{l}\text { OSI protocol } \\
\text { layer }\end{array}$ & \multicolumn{3}{|c|}{$\begin{array}{l}\text { Framework nanoscale } \\
\text { component }\end{array}$} \\
\hline Application & \multicolumn{3}{|c|}{-} \\
\hline Presentation & \multicolumn{3}{|l|}{-} \\
\hline Session & \multicolumn{3}{|l|}{-} \\
\hline Transport & \multicolumn{3}{|l|}{-} \\
\hline Network & & Field & \\
\hline Data Link & Specificity & \multirow[b]{2}{*}{ Motion } & \\
\hline Physical & $\begin{array}{l}\text { Message } \\
\text { Carrier }\end{array}$ & & Perturbation \\
\hline
\end{tabular}

Simulator 3 (NS-3) platform Its purpose is to integrate all the aforementioned components with their corresponding interfaces in a common platform, establishing a starting point for exploiting the benefits of the IEEE 19061 standard We note that this simulator was launched at the same time as the standard and no updates have been released, so an effort to keep it updated is required to prevent it from becoming obsolete

\section{IEEE 1906.1 STANDARD ACHIEVEMENTS AND FURTHER CHALLENGES FOR EM COMMUNICATIONS}

Once a general overview of the IEEE 19061 standard has been introduced, we identify different aspects which might be covered in forthcoming standardization activities to prevent future developments from diverging excessively As the first in the series, the standard lays the foundations of nanoscale communication networks However, some issues should be better detailed in order for it to serve as a solid reference for nanocommunications We explore these issues and challenges, separating them into six topics, namely: (i) device architecture, (ii) energy harvesting and storage, (iii) energy consumption, (iv) signal-to-noise ratio (SNR) and channel characterization, (v) data link communication techniques, and (vi) routing techniques Table 3 summarizes the identified gaps in the current standard, highlighting the benefit of filling each one, and associates them with their corresponding recommendations

\subsection{DEVICE ARCHITECTURE}

Some factors of a nanoscale communication protocol directly depend on the electronic architecture on which the communication system is built Among these factors we find energy consumption, available memory, and data processing capacity Thus, the standardization of a basic device architecture required to support an EM communication system is recommended From now on, we denote the device as nanodevice, although its dimensions, according to the open literature, may be at the microscale

The standard scarcely addresses this aspect in the definition of the concept "nanoscale communication network" Specifically, the only requirement for a nano-networking 


\section{CHAPTER 5. THE IEEE 1906.1 STANDARD: SOME GUIDELINES FOR}

FUTURE NORMALIZATION IN EM NANOCOM.

device is that its communication system must include one or more essential components, sized at nanometers in at leas one dimension By contrast, the scope of the standard seems to go beyond classical wireless electronic devices (its title refers to "nanoscale and molecular communication"), but it does not preclude them This lack of specification can be explained by the difficulty of giving a general definition including two types of communication (molecular and EM), which are based on totally different physical principles In this manner, published works employing EM communication systems at $\mathrm{THz}$ frequencies built upon an electronic device in the range of micrometers [6]-[8] are covered by the standard Thus, a basic definition related to device architecture should be considered in future version of the standard, in order to establish boundaries and exclude excessively large devices from becoming the core of a nanoscale communication network

As a first step, the conceptual devices presented in [6], [9] could establish a base upon which researchers could design novel nanoscale communication networks, even though more efforts are required to reach a definitive standardization Towards this end, a trade-off between size and technical viability should be considered to propose realistic electronic platform Some reference values for available memory, processing capacity, and energy consumption based on feasible technologies can be found in [6]

\subsection{ENERGY HARVESTING AND STORAGE}

Restrictions on the amount of available energy in each nanodevice have an important impact on the communication scheme The most widely accepted solution for powering nanodevices entails the use of piezoelectric nanogenerators, which are able to convert mechanical strains into electric energy, together with a capacitor to store the energy harvested The use of a capacitor, instead of a traditional battery, is motivated by its high storing capacity per unit of area, which allows better miniaturization Concretely, the capacitor employed for the energetical study in [7] reaches a capacitance of $9 \mathrm{pF}$ per $\mu \mathrm{m}^{2}$, which for a capacitor of $1000 \mu \mathrm{m}^{2}$ results in the maximum energy stored being 800 pJ Nevertheless, the main drawback to this piezoelectric nanogenerator is the scarce amount of energy collected per unit of area, which strictly limits the communication capabilities of nanodevices In addition, the available energy depends on the physical medium in which nanodevices are deployed (if nanodevices take advantage of environmental motion, the energy harvested will be greater than in a static scenario)

Thus, more effort should be devoted to characterizing the performance of a nanoscale energy generator and its operating conditions, studying its performance (in energy harvesting rate and maximum energy storing capacity) as a function of the area or the external energy source For instance, the use of ultrasound as an energy source could be standardized as well Once the study of these variables has led to realistic and optimized results, they could be standardized to set a common solution for powerin nanodevices Thus, we believe that the standard should include a reference powering solution for nanodevices, considering (i) energy harvesting restrictions due to their tiny size (pointing to the available area in the nanodevice to integrate the nanogenerator) and (ii) the environment under study So, this reference model would establish a more solid starting point to quantifying important aspects of communication, such as coverage area, the size of the message to be transmitted, and so on, which could be consistently used to develop feasible communication protocols

\subsection{ENERGY CONSUMPTION}

In this regard, the standard provides a theoretical upper boundary on quantum computation $\left(6 \times 10^{33}\right.$ operations per second per joule of energy) when describing the perturbation component Nevertheless, to the best of our knowledge, no work on EM nanocommunications makes use of quantum computation to estimate the energy consumption of a nanonetwork This is due to the fact that quantum computers are at too early a stage to be implemented at the nano, or even micro, scale In addition, energy is also mentioned in the Information and Communication Energy metric, providing the analytic expression to measure the energy per bit required to transmit a message

However, in our opinion, the energy required to transmit a bit gains importance in nanonetworks due to two main factors: (i) the huge path losses in the $\mathrm{THz}$ band and (ii) the scarce amount of energy stored in a nanocapacitor As can be seen in Figure 1, the transmission energy per bit when employing a TS-OOK modulation [8] has a critical impact on the total power consumption When the transmission energy remains low, around $1 \mathrm{aJ}$, the percentage of power with respect to the total is lower than $1 \%$, whereas for transmission energy at a higher level, up to $500 \mathrm{pJ}$, this value rises to $99 \%$ All these energy transmission values have been employed in different works published in the related literature [4], [10]-[13] This high variability between energy transmission values in nanonetworks highlights the need for standardization In addition,

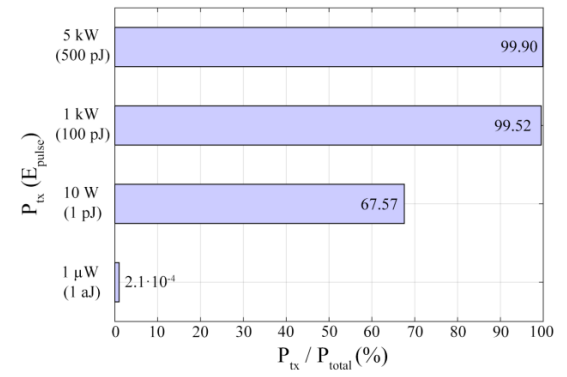

Figure 1 Ratio between the power required for communication and the total power consumption for different values of transmission power taken from the literature 


\section{CHAPTER 5. THE IEEE 1906.1 STANDARD: SOME GUIDELINES FOR}

88

standardization of energy values should attract even more attention when human bodies are involved, since the high transmission power envisaged to overcome absorption losses in human tissues [14] might have some impact

\subsection{SNR AND CHANNEL CHARACTERIZATION}

Parameters related to the reception of EM waves, such as signal to noise ratio (SNR), are not addressed in the IEEE 19061 standard The SNR at reception is an important factor to consider in order to ensure robust and reliable nanoscale communications Although the standard deals with the channel capacity (computed using the Shannon theorem), calculating the upper boundary for the physical data rate, in the case of a low SNR value, the receiver would not be able to demodulate the radio signal

Channel characterization is another crucial aspect to deal with in order to advance towards feasible EM nanocommunications This points to continuing to analyze the behavior of different propagation environments at $\mathrm{TH}$ frequencies, including the human body [15] However, we consider this research to be parallel and complementary steps towards progress in the standardization efforts for developing communications at the nanoscale

Therefore, an SNR value recommendation should be taken into consideration by the standard, to set a threshold to appropriately demodulate the signal at the receiver Powe transmission and SNR, together with the path loss model characterizing each physical medium (e $\mathrm{g}$ human body tissues) would clearly determine the transmission range of each nanodevice for the scenario under study, which would be useful, for instance, in the planning of the required number of nanodevices deployed to cover a particular area

\subsection{DATA-LINK COMMUNICATION TECHNIQUES}

As can be observed in Table 2, the standard places the framework components specificity and motion in the data link layer In EM communications, these components are identified as signal radiation (motion) and antenna aperture in reception (specificity) -see Table 1- However, as EM nanoscale communication networks must contain a huge

Table 3 Suggested gaps to fill and recommended steps to take in the standardization of EM communications

\begin{tabular}{|c|c|}
\hline $\begin{array}{l}\text { Suggested gaps to fill in upcoming } \\
\text { standardization efforts for EM communications }\end{array}$ & Recommendations \\
\hline $\begin{array}{l}\text { Main characteristics of a potential nanodevice for } \\
\text { EM nanonetworks have not yet been addressed by } \\
\text { the standard It would be suitable to join } \\
\text { standardization efforts to encourage a faster } \\
\text { development of nanocommunications }\end{array}$ & $\begin{array}{l}\text { More detailed definition of nanoscale EM communication } \\
\text { devices Two separate chapters for molecular and EM } \\
\text { nanocommunication are suggested }\end{array}$ \\
\hline $\begin{array}{l}\text { The current version of the standard does not include } \\
\text { a reference powering solution/s for nanodevices, } \\
\text { which is of crucial importance to establish realistic } \\
\text { values for energy harvesting and consumption }\end{array}$ & $\begin{array}{l}\text { Definition of a general powering solution for nanodevices } \\
\text { Energy restrictions in both harvesting and consumption should be } \\
\text { better quantified to design a nanonetwork offering a real service }\end{array}$ \\
\hline $\begin{array}{l}\text { The standard would benefit from specifying } \\
\text { recommended values for power transmission because } \\
\text { it would better determine the transmission range of } \\
\text { nanonodes, the energy required to send information, } \\
\text { and ensure that the power employed is not harmful to } \\
\text { human health and the environment }\end{array}$ & $\begin{array}{l}\text { Standardization of power transmission within a defined range to } \\
\text { amend the enormous variation between values employed in the } \\
\text { literature }\end{array}$ \\
\hline $\begin{array}{l}\text { Together with the standardization of power } \\
\text { transmission, a SNR recommended range at } \\
\text { reception would help future researchers to validate } \\
\text { the coverage of nanodevices upon a common } \\
\text { standard }\end{array}$ & $\begin{array}{l}\text { Standardization of a SNR value range expected at reception to } \\
\text { calculate appropriate receiver sensitivity thresholds }\end{array}$ \\
\hline $\begin{array}{l}\text { The inclusion of data-link (layer 2) communication } \\
\text { techniques basic core adapted to the characteristics } \\
\text { of nanonetworks, along with a standardized packet, } \\
\text { would facilitate protocol interoperability }\end{array}$ & $\begin{array}{l}\text { Standardization of fundamental techniques referring to media } \\
\text { access control, addressing scheme, flow control, and error } \\
\text { detection }\end{array}$ \\
\hline $\begin{array}{l}\text { Equivalent layer } 3 \text { OSI reference model functions } \\
\text { should be addressed to improve interoperability of } \\
\text { routing techniques among nanonetworks }\end{array}$ & $\begin{array}{l}\text { Description of recommended routing procedures to allow multi- } \\
\text { hop, end-to-end communications }\end{array}$ \\
\hline $\begin{array}{l}\text { The standard does not include any recommendation } \\
\text { about the interconnection between a nanoscale } \\
\text { communication network and macroscale devices, } \\
\text { which could impact on other communication aspects }\end{array}$ & $\begin{array}{l}\text { Providing some recommendations or best practices about the } \\
\text { design and development of a link between the nano and } \\
\text { macroscale domains }\end{array}$ \\
\hline
\end{tabular}




\section{CHAPTER 5. THE IEEE 1906.1 STANDARD: SOME GUIDELINES FOR FUTURE NORMALIZATION IN EM NANOCOM.

number of cooperating nanodevices due to their extremely limited transmission range, some techniques are required to enhance the data transmission robustness between adjacent nanodevices Specifically, medium access control to arbitrate transmissions and avoid message collisions, flow control to encompass the bitrate of the communication link, or error detection mechanisms would be required In addition, the number of fields; control, payload, and foote field lengths of the reference message are not defined by the standard, which could lead to the design of different and even non-interoperable data link layers

Thereby, future versions of the standard should include some techniques to improve the data transmissio robustness between neighboring nanodevices We divide them into four subgroups: (i) media access, (ii) flow control, (iii) addressing, and (iv) error detection/correction Firstly, due to the very high density of nanodevices expected in nanonetwork deployment, straightforward media access control should regulate access to the radio channel, to manage simultaneous transmissions in the shared medium For instance, by using any type of randomized process (e g a backoff) to activate the nanodevice transceiver and listen to the medium, message collisions will be mitigated In the case that a medium access control technique is no employed, messages dispatched by neighbors could collide, corrupting a high percentage of the transmitted data Secondly, for the same reason, a flow control mechanism is essential to coordinate the communication between nanodevices For example, a simple acknowledgement reply to confirm the reception of a message, together with a time for retransmissions (when collisions occur) could be enough to control the traffic load in the network The retransmission of packets should be carefully designed, due to the scarce memory and energy of nanodevices Thirdly, the management of nanodevice identifiers (ID) in nanonetwork pose an important challenge due to the high amount of nanodevices comprising the network Different ID assignment methods have been proposed, which in part depend on the network topology employed On the on hand, the use of addresses can be optimized by dividing them into subranges, each one assigned to one cluster, which would act as a sub-nanonetwork This approach shrinks the number of bits required for addressing, thus reducing the energy employed for data transmission However, this architecture poses problems when the WNSN is not static, since clusters would change continuously Thi variability entails the reconfiguration of the WNSN addressing too frequently, which would result in an excessive waste of energy for nanodevices On the other hand, the assignment of a unique ID to each nanodevice has the advantage that it would not require reassignment However, the address could be excessively long if th number of nanonodes in the nanonetwork is too high Finally, error detection methods are mandatory to evaluate standard metrics such as sensitivity or specificity, since false positives must be properly detected Hence, considering all these communication aspects, a common protocol for nanonetworks should be included in future versions of the standard (focused on EM communication) to encourage the interoperability between nanoscale communication networks

\subsection{ROUTING TECHNIQUES}

Concerning the network layer, nanodevices may have to reply to a request from an external macroscale device or may need to immediately report new events to external end personnel Due to the very limited transmission range of nanodevices, this information flow would require the creation of multi-hop routes The IEEE 19061 standard establishes the field component as a part of the network layer and defines the element relay as a retransmitter of the message However, it does not cover the functionalities related to multi-hop, end-to-end communications or how the information flows to reach a remote terminal nanodevice This issue has an effect on the packet length, since additional headers (e g time to live [TTL]) may be required, having in turn, an impact on energy consumption In addition, the interconnection of a nanoscale communication network with the macro world should be tackled in future versions of the standard, since the mechanism to make it possible could have an impact on other communication aspects

\section{CONCLUSIONS}

The IEEE 19061 standard establishes a set of recommended practices with the aim of allowing researchers to advance in the development of effective nanoscale communication systems These general requirements involve two contrasting approaches On the one hand, they leave the door open to many different ideas, which with a field in its early stages, is a crucial advantage However, on the other hand, the standard seeks to set a framework to facilitate researchers working in the same direction, but excessively lenient requirements could render it ineffective This is due to the fact that researchers migh not be motivated to change their methodology and design to adapt them to the standard, since most of the prior and divergent works in nanocommunications comply with it This way, even though the current standard has helped to alleviate this problem and provides a significant step forward, more concreteness in future versions is necessary to really meet the purpose for which it was conceived Keeping this premise in mind, we have first reviewed the main body of the standard, highlighting those definitions, metrics, and components related to EM communications Then, we have identified and discussed potential gaps and offered possible guidelines for addressing each one, in order to enhance the feasibility and capabilities of EM nanoscale communications We also highlight the importance of dedicating efforts towards the standardization of the issues analyzed, to adapt all the communication features to the peculiarities of nanodevices

Pursuing this goal, due to the difficulty of combining molecular and EM communication paradigms, we propose the division of the standard into two branches, one focused on EM communications and the other specifically devoted to molecular nanoscale communications The result would 
be a suitable definition for each type of communication, which would provide a more complete standardization, encompassing the true dimension of communication networks at the nanoscale

\section{REFERENCES}

[1] I Llatser, C Kremers, A Cabellos-Aparicio, J M Jornet, E Alarcón, and D N Chigrin, "Graphene-based nano-patch antenna for terahertz radiation," Photonics Nanostructures - Fundam. Appl., vol 10, no 4, pp 353358 , Oct 2012

[2] J M Jornet and I F Akyildiz, "Graphene-based plasmonic nano-transceiver for terahertz band communication," in The 8th European Conference on Antennas and Propagation (EuCAP 2014), 2014, pp 492-496

[3] P Kulakowski, K Solarczyk, and K Wojcik, "Routing in FRET-Based Nanonetworks," IEEE Commun. Mag., vol 55, no 9, pp 218-224, 2017

[4] S F Bush, J L Paluh, G Piro, V Rao, R V Prasad, and A Eckford, "Defining Communication at the Bottom," IEEE Trans. Mol. Biol. Multi-Scale Commun., vol 1, no 1, pp 90-96, Mar 2015

[5] IEEE Recommended Practice for Nanoscale and Molecular Communication Framework IEEE Std 19061,2015

[6] S Canovas-Carrasco, A -J Garcia-Sanchez, F GarciaSanchez, and J Garcia-Haro, "Conceptual Design of a Nano-Networking Device," Sensors, vol 16, no 12, p 2104, Dec 2016

[7] J M Jornet and I F Akyildiz, "Joint Energy Harvesting and Communication Analysis for Perpetual Wireles Nanosensor Networks in the Terahertz Band," IEEE Trans. Nanotechnol., vol 11, no 3, pp 570-580, May 2012

[8] J M Jornet and I F Akyildiz, "Femtosecond-Long Pulse-Based Modulation for Terahertz Band Communication in Nanonetworks," IEEE Trans. Commun., vol 62, no 5, pp 1742-1754, May 2014

[9] J M Jornet and I F Akyildiz, "The internet of multimedia Nano-Things," Nano Commun. Netw., vol 3 , no 4, pp 242-251, 2012

10] J M Jornet, J Capdevila Pujol, and J Solé Pareta, "PHLAME: A Physical Layer Aware MAC protocol for Electromagnetic nanonetworks in the Terahertz Band," Nano Commun. Netw., vol 3, no 1, pp 74-81, Mar 2012

[11] P Wang, J M Jornet, M G Abbas Malik, N Akkari, and I F Akyildiz, "Energy and spectrum-aware MAC protocol for perpetual wireless nanosensor networks in the Terahertz Band," Ad Hoc Networks, vol 11, no 8, pp 2541-2555, 2013

[12] S Canovas-Carrasco, A -J Garcia-Sanchez, and J Garcia-haro, "A nanoscale communication network scheme and energy model for a human hand scenario," Nano Commun. Netw., vol 15, pp 17-27, 2018

[13] G Piro, G Boggia, and L A Grieco, "On the design of an energy-harvesting protocol stack for Body Area NanoNETworks," Nano Commun. Netw., vol 6, no 2, pp 74 84, Jun 2015

[14] G Piro, K Yang, G Boggia, N Chopra, L A Grieco, and A Alomainy, "Terahertz Communications in Human Tissues at the Nanoscale for Healthcare Applications," IEEE Trans. Nanotechnol., vol 14, no 3, pp 404-406, 2015

15] N Chopra, K Yang, J Upton, Q H Abbasi, K Qaraqe, M Philpott, A Alomainy, "Fibroblasts cell number density based human skin characterization at $\mathrm{THz}$ for in body nanonetworks," Nano Commun. Netw., vol 10, pp 60-67, Dec 2016

\section{BIOGRAPHIES}

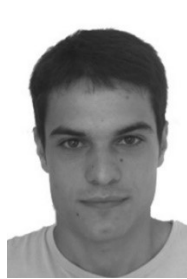

SEBASTIAN CANOVASCARRASCO (M'17) received the B S degree in telecommunication systems engineering and the M S degree in telecommunications engineering from the Universidad Politécnica de Cartagena, Spain, in 2014 and 2016, respectively, where he is currently pursuing the $\mathrm{Ph} \mathrm{D}$ degree His research interest includes the development of electromagnetic wireless nanonetworks and radiocommunications at the $\mathrm{THz}$ band

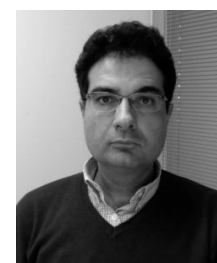

ANTONIO-JAVIER GARCIASANCHEZ received the $\mathrm{PhD}$ degree from the Technical University of Cartagena (UPCT), Spain, in 2005 Currently, he is an Associate Professor at the UPCT $\mathrm{He}$ is a (co)author of more than 70 papers, thirty of them indexed in ISI-JCR journals $\mathrm{He}$ has been the main head in several EU and national research projects, inventor/co-inventor of 11 patents or utility models, TPC/Chair in about forty Congresses/Workshops $\mathrm{He}$ is editor of several ISI-JCR journals

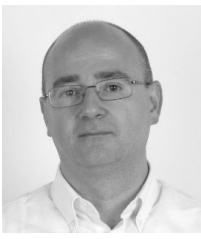

JOAN GARCIA-HARO (M'91) received the $\mathrm{MS}$ and $\mathrm{Ph} \mathrm{D}$ degrees in telecommunication engineering from the Universitat Politecnica de Catalunya, Barcelona, Spain, in 1989 and 1995 , respectively $\mathrm{He}$ is currently a Professor with the Universidad Politecnica de Cartagena (UPCT) He is author or co-author of more than 80 journal papers listed in the Journal Citation Reports $\mathrm{He}$ has been a visiting scholar at Queen's University at Kingston, Canada (1991-1992) and at Cornell University, Ithaca, USA (2010-2011) 
Chapter 6

\section{Optimal Transmission Policy \\ Derivation for IoNT \\ Flow-Guided Nano-Sensor \\ Networks}




\title{
Optimal Transmission Policy Derivation for IoNT Flow-guided Nano-sensor Networks
}

\author{
Sebastian Canovas-Carrasco, Ruben M. Sandoval, Antonio-Javier Garcia-Sanchez, and Joan Garcia-Haro
}

\begin{abstract}
By empowering the IoT with nanoscale communicato monitor complex environments in a nonintrusive way, giving rise to the Internet of Nano-Things (IoNT) paradigm. It is in one of these very complex and critical environments, the human ardiovascular system, where IoNT might stand out. However, he supervision of human health poses significant challenges:

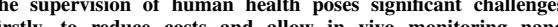
rstly, to reduce costs and allow in vivo monitoring nanensor networks, the number of deployed nano-routers must be mited, which could cause nano-nodes to undergo long out-ofcoverage periods. Lastly, nano-nodes rely on harvested energ rom the medium, which severely reduces the number of potential transmissions. To overcome these two problems, smart policies that direct devices on how to proceed with sensed events ar crucial. In this line, we propose a generic Markov Decisio Process (MDP) model which can be exploited to derive optimal transmission policies that are easily employed by nano-nodes. These policies maximize nano-node throughput and cope with the energy and coverage problems. We have also run a set of simulations to validate our proposal and to compare it to othe alternative policies. Results reveal that: (i) our policy systematically outperforms the rest of policies by a large margin; (ii) recis on pectwee (the Pyth to be easily adaptable to researchers' needs, making it easier for to be easily adaptable to researchers' needs, making it
future IoNT works to add intelligence to the network.
\end{abstract}

Index Terms-Internet of Nano-Things (IoNT), Markov Decision Process, Energy Harvesting, Terahertz Band

I. INTRODUCTION

$\mathbf{T}$ $\mathrm{HE}$ advancements in the emerging field of nanotechnology are leading to novel communication paradigms that re envisaged to revolutionize the Internet of Things (IoT). The connection of size-constrained devices, hereinafter referred a nano-nodes, to the Internet paves the way for the Internet of Nano-Things (IoNT) [1]. Nano-nodes composing this IoNT are in the scale of a few cubic micrometers, enabling non-invasive deployments in mediums where larger sensors would not meet the size requirements. One of the most prominent application fields is the human body, where the use of IoNT networks would significantly improve the effectiveness of disease diag nosis and treatment by monitoring health parameters in vivo, as shown in many works in the recent literature [2]-[6].

In this sense, an IoNT network especially designed to ope ate, for instance, within the circulatory system could provide

All authors are with the Department of Information and Communication , 2327-4662 O2016 IEEE Personal use is pes apct.es). republication/redistribution requires IEEE permission. See http://www.ieee.org/publications_standards/publications/rights/index.html

for more information

Digital Object Identifier: 10.1109/JIOT.2019.2906015 real-time measurements of blood parameters. As discussed in [7], accurately monitoring blood can be effectively used for disease detection in early stages, hence the special interest of employing IoNT for health monitoring. In this scenario, nanonodes will be in constant movement through the bloodstream, eading to a new type of IoNT network, which we refer to as low-guided nano-networks.

Flow-guided nano-networks should be designed considering he nano-node limitations determined by their reduced size, which make the adoption of the IoNT very challenging, as discussed in [8]. Firstly, the miniaturization of the antenna to be installed in a nano-node imposes the use of the erahertz band (1-10 THz) to communicate [3]. Inherently, these working frequencies lead to high path losses when the transmitted signal propagates through the medium, severely constraining the communication range. This handicap is even more restrictive when the medium through which the energy propagates has a high water content, e.g. animal and human body tissues and fluids [4], [9], [10]. In addition, the amount of energy that could be stored in a nano-node battery is extremely reduced and it cannot be manually replaced or recharged. As thoroughly analyzed for wireless body area networks [11], these factors entail the use of a piezoelectric nano-generator integrated into each nano-node to harvest energy from the environment [12], [13], providing a continuous but scarce source of energy.

A potential solution to reduce the impact of these limitations is a three-tier network architecture, following the hierarchy employed in different works [1], [14]-[17], in which three different kinds of devices compose the network. The lowest tier covers the smallest devices (i.e. nano-nodes), which sense the medium, and generate all the raw network data. Nano-nodes are characterized by their very limited computing capacity, which stems from their reduced size [13], [18]. In the middle tier, larger and less resource-constrained devices, called nano-routers, are in charge of gathering and aggregating data generated by nano-nodes and sending them to the upper ier (i.e. Internet gateway), which is directly connected to the Internet. This topology presents two main advantages: (i) it reduces energy consumption by releasing nano-nodes from heavy computing tasks, moving all the protocol complexity to larger and less energy-constrained devices; and (ii) it minimizes the distance of the communication link to the Internet gateway (mitigating the very high path loss) by a multi-hop transmission through the different tiers. Considering this network topology, a well-suited solution for a flow-guided network is the implantation of a nano-router infrastructure along the stream in which nano-nodes flow, enabling the interconnection between nano-nodes and the Internet gateway. 


\section{CHAPTER 6. OPTIMAL TRANSMISSION POLICY DERIVATION FOR IONT FGNN

However, the number of nano-routers deployed must be kept within reasonable values, since they are larger in size and more invasive devices than nano-nodes. This fact, together with the very short communication distance allowed for the nanonode/nano-router link, result in long out-of-coverage periods for nano-nodes. Under these conditions, nano-nodes must rely on optimal transmission policies that determine whether or not it is beneficial to store, transmit, or drop sensed events. Moreover, as energy is extremely limited in IoNT networks, such policies must always ensure that this scarce resource is managed intelligently. Considering the importance of flowguided nano-networks in enabling the application of IoNT to human health, in this work we have focused on minimizing the impact of these two issues (coverage and energy) on nano-network performance. Furthermore, by deriving optimal transmission policies, we also maximize the throughput of this kind of network. To summarize, the most significan contributions of this work are:

- We propose a flow-guided nano-network specially suite for (but not limited to) the human circulatory system where nano-nodes are confined within a well-defined stream.

- We derive a mathematical framework based on Markov Decision Processes (MDP) for the computation of smar transmission policies. This framework is flexible enough to be applied in a wide range of applications in which flow-guided networks could be employed.

- We apply this mathematical framework to a realistic case of study: a nano-network monitoring the human circulatory system. For this scenario, a detailed analysis of the communication link budget, energy harvesting, an power consumption rates is also carried out.

- We simulate the derived smart policies in a wide range of scenarios and compare it to other alternative transmission policies for validating the analytical model and highlighting its strengths. Based on these simulations, we also evaluate the impact on the nano-network throughput of (i) the precision in placing nano-routers, and (ii) thei required number.

- Finally, we release all the programmed Python scripts to foster the reproducibility of results and let researcher easily use or adapt the examples provided to their own needs.

The rest of the paper is organized as follows. The related work is presented in Section II. The mathematical framework for flow-guided networks is defined in Section III. Section IV is devoted to rigorously defining the case of study, describing all the parameters employed in the simulation. Section V compares the prioritized throughput of a simulated flow-guided network when: (i) our proposed MDP-based transmission policy is employed, and (ii) other intuitive and reasonable transmission policies are used. Then, the results obtained under different scenarios are compared to numerically evaluate the benefits of employing our MDP policy. Finally, Section VI concludes.

\section{RELATED WORK}

Deriving optimal transmission policies for macroscale IoT devices is a topic already studied in the literature [19]-[23. In [19], an MDP framework was used to derive a transmission policy suited to the scarce resources of the IoT devices unde study. This policy achieved improvements of up to $69 \%$ in network performance, significantly increasing the number of reported events. However, the proposed methodology canno be directly applied to IoNT, due to the radically different needs of nano-networks. Regarding IoNT, just a few works deal with this topic. In [24], the problem of optimizing the energy utilization in distributed nano-networks (when only nanonodes are considered) is modeled as a one-dimensional MDP This MDP reflects the energy stored in the nano-node battery, where the different states simply indicate energy levels. Thus, this work is devoted to optimizing the energy utilization in nano-nodes, but does not consider nor maximize the nanonetwork throughput. Although the mathematical analysis of the energy-optimization problem is very well presented, the energy harvesting process is over-simplified by considering it to be a simple stochastic energy-arrival process. Furthermore, it is assumed that there are always packets to be transmitted (packet storage or queuing is not considered), which might not hold true in situations where events are generated based on sensor readings (e.g. the obtained value above a certain threshold). In [18], an optimal transmission scheduling for fully-distributed IoNT networks was proposed. This algorithm allows nanonodes to make optimal decisions based on their state in each time slot and subject to a transmission deadline, that is, the packet must be sent before a given time. The mathematical model presented in that work is very rigorous, since the deadline derived ensures that nano-nodes are able to perpetually communicate by balancing energy consumption and energy harvesting. However, this algorithm is focused on reaching an optimal time resource allocation and not maximizing nanonetwork throughput. In addition, the proposed algorithm poses two limitations: (i) it was only evaluated for a low number of nano-nodes, concretely 8 and 10, but IoNT is envisaged to work with a substantially larger number of them; and (ii) it must run in every nano-node, which might be excessively complex for their limited computing capacity.

Regarding the use of Markov chains for IoNT, authors in [12] used them to analyze the effect of energy harvesting in nano-nodes on an IoNT network. The Markov chain proposed considered a non-linear energy harvesting rate and a packet generation rate following a Poisson distribution. Then, authors obtained the stationary distribution of the Markov chain, an relying on this, analyzed the probability of nano-nodes to be in each one of the available energy states for different packet generation rates. Although the work is well detailed and very useful for predicting the behavior of an IoNT network, it does not offer any transmission policy for nano-nodes based on their state, but rather assumes nano-nodes will always transmit events when possible. This hinders the performance of nanonetworks by accepting that it is always in the interest of nodes to report events (regardless of their importance and the energy level of the battery). 


\section{CHAPTER 6. OPTIMAL TRANSMISSION POLICY DERIVATION FOR}

Concerning flow-guided networks for the IoNT, authors in 10] proposed a three-tier IoNT nano-network designed to operate in the human circulatory system with a nano-router mplanted in the skin. In that work, nano-nodes transmit the generated data to a static nano-router when they pass through its coverage range. This nano-router sends the aggregated dat to an Internet gateway placed outside the body, concretely, in a smart wearable. However, the main limitation of this flow-guided nano-network is that only one nano-router is considered. This constraint severely restricts the capacity of the nano-network, since nano-nodes must wait until they are within the nano-router coverage range to transmit generated data. In addition, nano-nodes just send the latest generated event, without applying any smart transmission policy.

\section{Mathematical BACKGRound}

As indicated in Section I, the objective of the propose approach is to add intelligence to nano-networks in order to maximize the throughput, while considering both coverage an energy limitations. To this end, we have modeled the optima policy-derivation task as an MDP [25]. The reason for this is twofold: first, because of the exceptional ability of thi particular mathematical framework to model the throughputmaximization problem as an optimization task over discrete actions [26]; thus making it particularly suitable for deriving transmission policies (as explored by [27]). And second, because the derived policies can be fitted in tables, which are one of the most efficient data structures in terms of reading speed. Note that policies are derived offline, in regular computers, whereas such policies are ultimately installed and used by nano-nodes without needing additional processing tasks; hence the usefulness of a tabulated policy that provides extremely fast usage times.

As indicated above, MDPs lay the mathematical foundation for deriving optimal sequences of action in complex environments. Formally, MDPs are described in terms of the following constituent elements:

- There is an agent whose optimal behavior is to be found. An agent can be seen as an action-taking entity (in ou case, a nano-node)

- The state of such an agent can be fully encoded as vector $s$, where $s \in \mathbf{S}$. The state $s$ encompasses all the inner and outer properties of the agent

- In each state, the agent can take some action $a \in \mathbf{A}$ (e.g. transmit an event, drop it, etc.)

- Whenever an action $a$ is taken, the state of the agent evolves from $s$ to $s^{\prime}$, according to the transition model $T$ of the environment. The transition model reflects how the agent and the environment change as actions are taken.

- Furthermore, after taking an action $a$, the agent obtain a reward $r$ (which can be positive or negative). This is considered to be a feedback signal for the agent to learn.

- The transition model $\mathbf{T}$ does not need to be deterministic and can be modeled as $\mathbf{T}=f\left(s, s^{\prime}, a\right) \in[0,1]$-which indicates the probability of transitioning from state $s$ to state $s$ when an action $a$ is taken-.
- The reward $r$ that the agent perceives can be modeled as a function of the state and the action taken, that is, $r(s, a)=f(s, a) \in \mathbb{R}$

MDP-solving algorithms derive what are called policies, represented by $\pi$. A policy, in its simplest form, is a mapping between states and actions, i.e. $\pi: \mathbf{S} \rightarrow \mathbf{A}$. When the agen is in a particular state, it can derive the action to take by evaluating the policy in that state. As indicated above, one of the simplest (and computationally efficient) ways to store a policy is by means of a table. Each key of the table represent a state, and the value for such an entry, the action prescribed by the tabulated policy.

The final goal of MDP-solving algorithms is to find the optimal policy $\pi^{*}$, maximizing the accumulation of rewards $(r)$ over an infinite time period (i.e. the entire lifetime of the agent). To make this sum converge, a discount rate $\gamma \in[0,1)$ or future rewards is usually incorporated [28]. Aside from making the sum computable, this discount rate also illustrates he fact that agents prefer immediate rewards over distan future rewards. Eq. 1 indicates how $\pi^{*}$ (the optimal policy) is computed as the policy that maximizes the sum of discounted rewards:

$$
\pi^{*}=\underset{\pi}{\arg \max }\left(\sum_{\delta=0}^{\inf } \gamma^{\delta} \cdot r\left(s_{\delta}, a_{\delta}\right)\right),
$$

where $\delta$ is a particular time slot, in which the agent is in state $s_{\delta}$ and takes action $a_{\delta}$. Note that Markovian systems operate in a fully discrete fashion where both time and distances are discretized. Time is divided into time slots of length $T$ second and distances into slices of $S$ meters. Note that $T$ and $S$ can be arbitrarily small to allow for very precise models.

At the beginning of each time slot $\delta$, nano-nodes are assumed to sense their environment and determine if an event $e$ needs to be reported. If so, a packet containing the required information of this event is composed and sent. Naturally, events could have different priority levels based on the detected physical parameter. For example, if a very high glucose concentration is detected in a diabetic patient, the maximum priority could be assigned to the event that reports on such a state. Conversely, if a mild decrease in hemoglobin levels is noticed, a lower priority event can be generated.

Thus, let us define $P$ different types of events $\mathbf{E}=$ $\left(e_{1}, \ldots, e_{P}\right)$, each with a different priority $\mathbf{G}=\left(g_{1}, \ldots, g_{P}\right)$ and probability of being generated $\Theta=\left(\theta_{1}, \ldots, \theta_{P}\right)$. Having different generation probabilities reflects the fact that, for instance, it is not as common to sense a very high increase in glucose concentration as it is to sense a mild decrease in hemoglobin levels. Furthermore, for the sake of generalization, we also consider that events might need a different amount of information (bits) to be transmitted $\mathbf{L}=\left(l_{1}, \ldots, l_{P}\right)$. More precisely, events might require packets of different length to convey their information.

When an event is generated, nano-nodes obtain the action to take from the pre-computed tabulated policy. In the context of nano-networks, we consider that nano-nodes can take one of the following three actions $\mathbf{A}=\{$ drop, store,transmit $\}$. That is, nodes can either drop, store or transmit a generated 


\section{CHAPTER 6. OPTIMAL TRANSMISSION POLICY DERIVATION FOR IONT FGNN

event. If the optimal action is to transmit the event, some energy is consumed $C_{T X}^{e}$ (based on the length, in bits, of the packet associated to the event $e$ ) and some reward $r \in \mathbb{R}$ is obtained. A more thorough discussion on power consumption is presented in Section IV. To maximize the prioritized throughput achieved (the metric that we want to maximize), the reward is shaped to be proportional to the priority of the event $e$ and the amount of bits transmitted, that is, the length of such an event $L(e)$ (see Eq. 2):

$$
r(e)=G(e) \cdot L(e),
$$

where $G(e)$ indicates the priority of the event being reported and $L(e)$ the length of the associated transmitted packet. Note that as we consider a flow-guided network, nano-nodes can only transmit packets when they are under the coverage range of a nano-router. Conversely, if the prescribed action is to store the generated event (e.g. because an event worth transmitting has been generated when the nano-node is not in coverage range of any nano-router), we consider that certain energy is consumed to store such an event in memory. Precisely, due to having considered RAM memories [13] as the main volatile storage units, some energy $\left(C_{K P}\right)$ is drawn in every time slot $(T)$ to keep the event in memory (as the circuits to powe the RAM need constant energy). Note, that in order to eithe transmit or store an event $e$, enough energy must be stored in the nano-node battery $\left(C_{T X}^{e}\right.$ or $C_{K P}$ respectively), otherwise the event will be forcedly dropped. Finally, if no event is generated or the optimal action is to drop it, we assume that the nano-nodes will perform a drop action.

As with time and space, energy is also discretized. Each nano-node presents a battery level $q$ within $\left[0, Q, 2 Q, \ldots, Q_{M A X}\right]$, where $Q_{M A X}$ indicates the maximum capacity of the battery, and $Q$, the chosen energy-quantization value. Similarly, in each time slot, some $q_{h}$ units of energy are recharged as a result of the energy harvesting process (with $q_{h}$ being a multiple of $Q$ and $\frac{q_{h}}{T}$ the effective energy harvesting rate). Since the recharge rate of a capacitor, the constituent element of the battery, is not constant and depends on its actual charge level, $q_{h}$ is characterized as a (discretized) function of $q$, that is, $q_{h}(q)=f(q)$

Regarding the movement of nano-nodes through the guided medium, they are considered to have moved a multiple $k$ of $S$ at the beginning of each time slot (with $k \in \mathbb{Z}^{+}$). This is equivalent to saying that their speed $(V)$ is equal to $V=k \cdot \frac{S}{T}$. Note that $k$ can be a function of time or space to reflect changing speed mediums or even vary stochastically to allow for complex blood flow behaviours. However, in Section IV, specific example for constant speed is described. As nanonodes traverse the guided medium, they successively enter (and leave) the coverage range of nano-routers. We conside that nano-routers are less energy-constrained devices capable of announcing their presence by transmitting beacons. These beacons are received by nano-nodes (when they are within the coverage of a nano-router), so it is suppose that their packets will be received. Since it is unfeasible for nano-nodes to accurately know their exact position with respect to the whole guided medium, we take advantage of this beaconing system to endow them with some concept of location. In this way, and although a nano-node cannot know when it will be within the coverage range of the next nano-router, it can estimate such time by considering the separation between nano-routers and how far it is from the last nano-router in coverage. As beacons need not be decoded, we reasonably assume that enough energy reaches the nano-node for them to be passively detected without a pre-amplification stage. Therefore, no (or negligible) energy consumption is required to detect the presence of nano-routers.

Once all the variables have been defined (see Table I), the three-dimensional Markov chain associated to the MDP can be presented. This chain represents the state $s$ of the nano-node and how it evolves as it changes from one state to another. A representation of such a chain and its axes are shown in Fig. 1.

- On the $\mathbf{X}$ axis, the battery level of the nano-node is considered; ranging from 0 (battery depleted) to $Q_{M A X}$ (battery completely full). The dynamics of this axis are simple: when transmitting or storing events, some energy is consumed $\left(C_{T X}^{e}\right.$ or $C_{K P}$ respectively), whereas at the beginning of the time slot some energy is harvested, determined by $q_{h}(q)$.

- On the $\mathbf{Y}$ axis, the event-generation state is modeled; that is, the type of event that has been generated. Note that this axis will represent the $P$ different types of events plus the state of not having generated any event (denoted as $e_{0}$ hereinafter). When an event is transmitted or discarded, the nano-node changes to $e_{0}$, whereas at the beginning of each time slot, some event $e$ may have been generated (with its corresponding $\theta$ probability).

- Finally, on the $\mathbf{Z}$ axis, the distance of the nano-node from the last traversed nano-router is reflected. At the beginning of the time slot, nano-nodes move forwar (some $k$ steps). If they receive a beacon signal, they are considered to be within nano-router coverage, and thus, return back to state 0 (i.e. they are $0 \cdot S$ meters away from a nano-router). The maximum inter-nano-router distance is denoted as $S_{M A X}$

As can be seen, the full state of any arbitrary nanonode can be expressed by (1) its battery level, (2) its even generation state, and (3) the distance from the last nanorouter in coverage. On the other hand, this state is altered (i) when an event $e$ is generated, (ii) at the beginning of each time slot, when the nano-node has harvested some energy and moved forward in the guided medium, or (iii) when an action is taken (namely, drop, store or transmit). The only element under the direct control of the nano-node and subject to optimization is the action to be taken in each state. This is precisely the objective of the MDP algorithm: to derive the optimal transmission policy for a nano-node.

Considering the three-dimensional Markovian chain depicted in Fig. 1, we can now resort to very efficient MDP algorithms, such as Q-learning [29] to determine the best action to take in each state; that is, the optimal policy. Q learning is an iterative MDP-solving algorithm that derives the quality of any given state-action pair $\mathbb{Q}(s, a)$; that is, the 
CHAPTER 6. OPTIMAL TRANSMISSION POLICY DERIVATION FOR

IONT FGNN

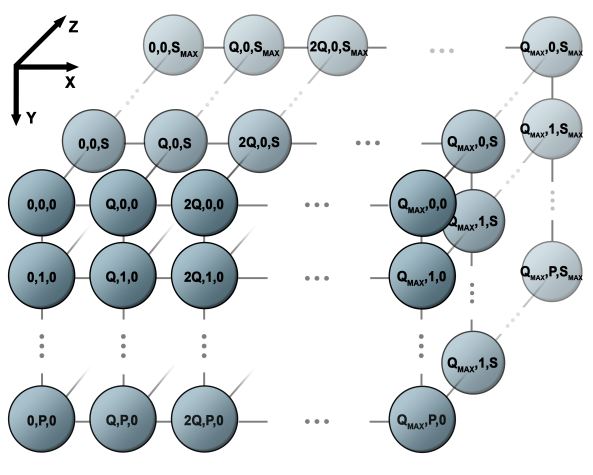

Fig. 1: Three-dimensional Markovian model. The $\mathbf{X}$ axis indicates the battery level of nano-nodes (from 0 to $Q_{M A X}$ ). The $\mathbf{Y}$ axis indicates the type of event generated. The $\mathbf{Z}$ axis denotes the distance from the last traversed nano-router. Transitions between states have not been drawn for the sake of clarity.

appropriateness of taking action $a$ in any given state $s$. By iteratively updating the estimation of $\mathbb{Q}$ with Eq. 3, the quality of the optimal policy $\pi^{*}$ increases.

$\mathbb{Q}^{\text {new }}(s, a) \leftarrow(1-\alpha) \cdot \mathbb{Q}(s, a)+\alpha \cdot\left(r(s, a)+\gamma \cdot \max _{a} \mathbb{Q}\left(s^{\prime}, a\right)\right.$

The parameter $0<\alpha \leq 1$ is a learning rate that determine how much of the newly-acquired information is incorporate to the current estimation of $\mathbb{Q}$.

This optimal policy $\pi^{*}$ is derived so as to maximize the average obtained reward. Due to the way the reward $r(s, a)$ is shaped, this is equivalent to maximizing the prioritized throughput of nano-nodes. As the transmission policy is a simple mapping between states and actions, it can be effectively stored in a table; which in turn, would be programmed into the memory of the nano-nodes before deploying them Note that, as stated above, the utilization of tabulated policie entails a great reduction in computational requirements, which is of special interest if one considers how hardware constrained nano-nodes are.

\section{CASE Study}

In the following paragraphs, a case study of a flow-guided nano-network is thoroughly defined, using realistic and acknowledged values for all parameters employed, to evaluate the performance of the transmission policy derived from the MDP framework. Concretely, this case study consists of a nano-network constantly monitoring the health of a human being. This reference scenario is widely cited in the literature [2], [10], [30]-[32] and has gained momentum in the research community, thanks to the capacity of nano-networks to monitor important aspects of human health in a non intrusive way. Exploiting the extremely restrained size of nano-nodes, we assume that thousands of them are injected into the circulatory

\begin{tabular}{cc}
\hline Parameter & Description \\
\hline$s$ & State of the nano-node \\
$a$ & Action taken by the nano-node \\
$\mathbf{T}$ & Transition model of the MDP \\
$r$ & Reward obtained \\
$\pi$ & Transmission policy \\
$e$ & Generated event \\
$p$ & Type of event \\
$\gamma$ & Discount rate \\
$G(e)$ & Priority of event $e$ \\
$L(e)$ & Length of event $e$ \\
$\alpha$ & Learning rate \\
$S$ & Slice of space \\
$T$ & Slot of time \\
$\lambda$ & Event-generation rate \\
$q$ & Battery level \\
$Q_{M A X}$ & Maximum capacity of the battery \\
$C_{T X}^{e}$ & Energy consumption to transmit event $e$ \\
$C_{K P}$ & Storage energy consumption
\end{tabular}

TABLE I: Abbreviations employed throughout Section III.

system, whereas nano-routers are implanted directly over the vein as depicted in Fig. 2. Note that we consider that information flows strictly from nano-nodes to nano-routers (although the mathematical framework presented in the previous section does not impose any constraint on this).

Thus, the MDP formulation described in Section III is applied to this scenario. The objective of this study is twofold: firstly, to assess the benefits of deriving optimal policies via the MDP framework by comparing the obtained results with other intuitive transmission policies. Secondly, to apply the mathematical model to a particular example so researchers can easily use or adapt the example provided to their own needs, as our implemented Python code is made publicly available [33].

\section{A. Event-Generation Parameters}

As aforementioned, we let nano-nodes travel through the body sensing different types of events with different priorities. Specifically, we distinguish two types of events: low levels of hemoglobin (denoted as $e_{1}$ ) and high levels of glucose (denoted as $e_{2}$ ). Given the potential dramatic effects of quick rises in the glucose levels for diabetic people, we assign priorities $g_{1}=1$ for low-hemoglobin events and $g_{2}=5$ for high-glucose events. It should be noted that the assignment of priorities is made in order to highlight the importance of high-priority events without neglecting low-priority events. For instance, if the priority of high-priority events is excessively high, the reward associated with sending a low-priority event would be so low that most of them would be discarded. Conversely, when different events have similar priority values, the concept of priority is blurred, with both events being discarded with nearly the same probability. However, it should be observed that these priorities can be tuned for each application to be adapted to the different events generated, or alternatively, dynamically-generated approaches (such as the one explored in [34]) could be employed. Regarding the length of the packet generated for each event, we let $L\left(e_{1}\right)=64$ bits and $L\left(e_{2}\right)=128$ bits, so both improved reliability and 


\section{CHAPTER 6. OPTIMAL TRANSMISSION POLICY DERIVATION FOR IONT FGNN

\begin{tabular}{cc}
\hline Scenario & $\Lambda$ \\
\hline Low-Generation & $\lambda_{1}=0.66 \%, \lambda_{2}=0.33 \%$ \\
Mid-Generation & $\lambda_{1}=2 \%, \lambda_{2}=1 \%$ \\
High-Generation & $\lambda_{1}=6 \%, \lambda_{2}=3 \%$
\end{tabular}

TABLE II: Event-generation probabilities per time slot for the three different scenarios studied in Sections V-A and V-B

higher resolution in their readings can be added to glucose events. These packet lengths are short enough to be stored and transmitted by nano-nodes, but sufficiently large to include the header of an ad-hoc protocol, which has not yet been implemented for flow-based nano-networks and is therefore beyond the scope of this work. In addition, these packet lengths are in line with those employed in the literature [10], [12], [35]. With respect to the $\Lambda$ vector, we explore different cases in which events are generated with different probabilities, but generally speaking, we consider that lowpriority events (hemoglobin readings) are twice as likely as high-priority ones (glucose events): $\lambda_{1}=2 \cdot \lambda_{2}$. The specific event-generation probabilities are detailed in Table II.

As indicated in [13], the state of the art for nano-device manufacturing is still far from a reality. Among the presen challenges, we highlight those related to accurately positioning such devices in human tissues. For that purpose, instead of homogeneously distributing nano-routers throughout the body, we assume that precision in placing them is finite. Therefore, we let the distance between nano-routers $\left(D_{N R}\right)$ follow a uniform distribution: $D_{N R}=U\left[D_{N R}^{*}-\delta, D_{N R}^{*}+\delta\right]$. Tha is, we aim to place nano-routers exactly every $D_{N R}^{*}$ meter (the desired distance), but we do so with some imprecision $\delta$. This imprecision is thoroughly analyzed to see how it might impact the performance of the derived transmission policies. Interestingly, the average distance between nanorouters $\left(D_{N R}^{*}\right)$ is also varied to derive conclusions on how to save capital expenditures in future nano-network deployments.

\section{B. Scenario Description}

For the sake of simplicity, we assume that nano-nodes trave through the human body in a non-turbulent blood stream at constant speed of $V=10 \mathrm{~cm} / \mathrm{s}$ (a value consistent with the human circulatory system [36]). Since $V$ is constant, we set $k=1$. In order to determine the coverage range $(C R)$ of nanorouters, we consider the following: a sensitivity value of $S E=$

$-130 \mathrm{dBm}$ (a conservative value [37]), path losses of $P L=$ $130 \mathrm{~dB} / \mathrm{mm}$ (taken from a detailed analysis of path losses in blood [10]), and energy per pulse of $0.0001 \mathrm{pJ}$. This energ per pulse translates into a transmission power of $P_{T X}=1$ $\mathrm{mW}(0 \mathrm{dBm})$ if an On-Off keying modulation with pulses of $100 \mathrm{fs}$ is considered [38]. Using Eq. 4, we can easily derive that the maximum coverage range of nano-routers is $C R=$ $\mathrm{mm}$. All these variables have been specified in Table III.

$$
\begin{aligned}
S E & \geq P_{T X}-P L \\
-130 \mathrm{~dB} & \geq 0 \mathrm{dBm}-130 \mathrm{~dB} \cdot \operatorname{dist}(\mathrm{mm})
\end{aligned}
$$

We consider a vein height of $0.8 \mathrm{~mm}$ (a typical value as described in [36]) and let $S=1 \mathrm{~mm}$, a sufficiently small value for the quantization of the $Z$ axis. As we have defined

\begin{tabular}{cc}
\hline Parameter & Value \\
\hline$S$ & $1 \mathrm{~mm}$ \\
$T$ & $0.01 \mathrm{~s}$ \\
$Q$ & $0.3 \mathrm{fJ}$ \\
$Q_{M A X}$ & $64 \cdot Q=19.2 \mathrm{fJ}$ \\
$G$ & $g_{1}=1, g_{2}=5$ \\
$L$ & $L\left(e_{1}\right)=64 \mathrm{bits}, L\left(e_{2}\right)=128$ bits \\
$\mathrm{V}$ & $10 \mathrm{~cm} / \mathrm{s}$ \\
$\mathrm{CR}$ & $1 \mathrm{~mm}$ \\
$V_{g}$ & $0.2 \mathrm{~V}$ \\
$\Delta \mathcal{C}$ & $3 \mathrm{pC}$ \\
$f$ & $1 \mathrm{~Hz}$ \\
$C_{T X}^{e_{1}}$ & $11 \cdot Q=3.2 \mathrm{fJ}$ \\
$C_{T X}^{e_{2}}$ & $21 \cdot Q=6.4 \mathrm{fJ}$ \\
$C_{K P}$ & $8 \cdot Q=2.4 \mathrm{fJ}$
\end{tabular}

TABLE III: Values employed in the Case Study.

$V, S$, and $k$, we can now derive $T$ (the quantization value of time); as $V=k \cdot \frac{S}{T}$. Using this expression, we have that $T=0.01 \mathrm{~s}$.

\section{Energy Harvesting and Consumption Analysis}

As indicated in Section III, nano-nodes are considered to have harvested some energy at the beginning of each time slot, reflected as a change of state along the $\mathrm{X}$ axis in the MDP. These transitions are modeled by $\lambda_{h}(q)$, which defines the energy-harvesting rate as a function of the actual energy level. Therefore, a comprehensive derivation of its expression is required. As mentioned in Section I, nano-nodes employ energy-harvesting techniques based on piezoelectric nanogenerators to power their circuitry. From the energy analysis of nano-networks developed in [39], we derive the expression of the energy harvested by a piezoelectric nano-generator $(q)$ as a function of: (i) time ( $t$ ) and (ii) the maximum storable energy in a nano-node capacitor $\left(Q_{M A X}\right)$ :

$$
q(t)=Q_{M A X} \cdot\left(1-e^{\left(\frac{t \Delta c f V_{g}}{2 Q_{M A X}}\right)}\right)^{2}
$$

where $\Delta \mathcal{C}$ stands for the charge (in coulombs) generated in a compress-release cycle of the piezoelectric nano-generator, $V_{g}$ is the voltage induced by the nano-generator in a compressrelease cycle, and $f$ is the frequency of compress-release cycles. Following the values employed in the literature [12], [39], we set $\Delta \mathcal{C}$ and $V_{g}$ equal to $3 \mathrm{pC}$ and $0.2 \mathrm{~V}$, respectively, for a nano-generator of $500 \mu^{2}$. It should be noted that these parameters depend on the technology employed, so their values can be adapted to future nano-generators. As nano-nodes flow through the bloodstream, the frequency of the compress-release cycles matches the heart rate, that is, approximately 1 cycle per second $(1 \mathrm{~Hz})$.

From Eq. 5, we isolate the variable $t$ to obtain the time required to harvest an amount of energy $q$

$$
t(q)=-\frac{2 \cdot Q_{M A X}}{V_{g} \cdot \Delta \mathcal{C} \cdot f} \cdot \ln \left(1-\sqrt{\frac{q}{Q_{M A X}}}\right) .
$$


CHAPTER 6. OPTIMAL TRANSMISSION POLICY DERIVATION FOR

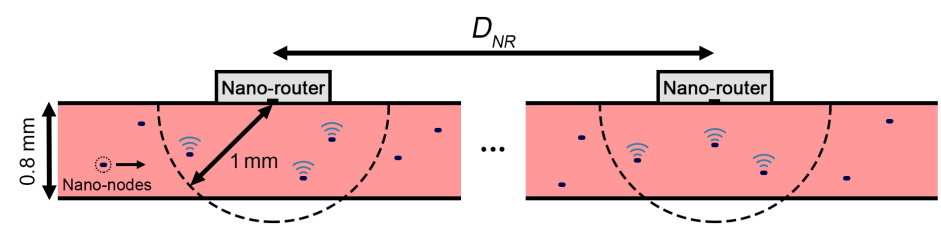

Fig. 2: Flow-guided nano-network architecture. Nano-nodes (indicated in the figure as dots or radiating dots when in nano-router coverage) travel through the circulatory system, entering and leaving nano-router coverage range.

The expression of the energy-harvesting rate as a function of time $\left(\lambda_{h}(t)\right)$ can be obtained from the time derivative of $q(t)$ :

$\lambda_{h}(t)=\frac{\partial q(t)}{\partial t}=V_{g} \cdot \Delta \mathcal{C} \cdot f \cdot\left(1-e^{\left(\frac{t \Delta \mathcal{C} f V_{g}}{2 Q_{M A X}}\right)}\right) \cdot e^{\left(\frac{t \Delta \mathcal{C} f V_{g}}{2 Q_{M A X}}\right)}$

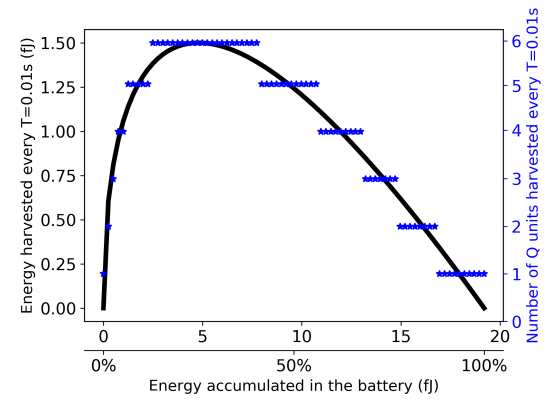

Finally, we compute the expression of the energy-harvesting rate (in joules per second) as a function of the actual level of energy, by substituting Eq. 6 in Eq. 7:

$$
\lambda_{h}(q)=\Delta \mathcal{C} \cdot f \cdot V_{g} \cdot \sqrt{\frac{q}{Q_{M A X}}} \cdot\left(1-\sqrt{\frac{q}{Q_{M A X}}}\right) .
$$

After each time slot of $\mathrm{T}$ seconds, the amount of energy that the nano-node will have harvested $\left(q_{h}\right)$ can be finally expressed as the product of the harvesting rate $\left(\lambda_{h}(q)\right)$ and the duration of such a time slot $(T): q_{h}(q)=\lambda_{h}(q) \cdot T$-plotted in Fig. 3 with a solid black line-.

To incorporate the energy-harvesting behavior of nanogenerators into the MDP, we have to discretize the inherently continuous values of $q_{h}(q)$. To this end, we have employed 6 levels of discretization, linearly quantizing the full range of values of $q_{h}(q)$ in one of the six available levels (shown as blue asterisks in Fig. 3). The number of levels is a tunable design value and has been chosen to provide us with enough precision without sacrificing speed in the derivation of the optimal policy. Therefore, the minimum harvestable amount of energy, $Q$, is equal to $Q=\frac{\max q_{h}(q)}{6}=0.3 \mathrm{fJ}$.

We assume a very conservative value for the maximum storable energy $\left(Q_{M A X}\right)$ in nano-node batteries of $19.2 \mathrm{f}$. [12]. Considering $Q=0.3 \mathrm{fJ}$, we get that $Q_{M A X}=64 \cdot Q$ When fully charged, the battery of nano-nodes could provide enough energy to transmit three events of 128 bits, or alternatively, six events of 64 bits. This is derived from considering energy per pulse $\left(E_{P}\right)$ of $0.0001 \mathrm{pJ}$ (or $0.1 \mathrm{fJ}$ ) and the use of an On-Off keying modulation. In this manner, the energy expected to be consumed in transmitting an event $e$ is given by:

$$
C_{T X}^{e}=L(e) \cdot W \cdot E_{P},
$$

where $W$ stands for the probability of sending a pulse, since the transmission of a logical ' 1 ' is performed by sending a pulse, whereas a logical ' 0 ' is sent as silence. On average, bits ' 1 ' and ' 0 ' are normally expected to be evenly distributed, so we assume $W=0.5$ [12]. Therefore, we have that $C_{T X}^{e_{2}}=$ $6.4 \mathrm{fJ}\left(\right.$ or $21 \cdot Q$ ) for $e_{2}$ and $C_{T X}^{e_{1}}=3.2 \mathrm{fJ}\left(\right.$ or $11 \cdot Q$ ) for $e_{1}$

Fig. 3: The solid black line represents $q_{h}$ as a function of the energy accumulated in the battery, expressed both in linear units (fJ) and as a percentage of $Q_{M A X}$. The blue asterisks are the quantized version of $q_{h}$, where the quantization value is $Q=0.3 \mathrm{fJ}$.

Regarding the energy consumption of the RAM memory, we have considered $C_{K P}=2.4 \mathrm{fJ}$ ( or $8 \cdot Q$ ), a value in line with those found in literature [40].

\section{Results}

Using the SimPy [41] discrete-event simulator, we have simulated the nano-network described in the previous section. To this end, we have considered a closed-loop flow-guided medium (simulating the cardiovascular system) and employed the variables in Table III to simulate 6000 nano-nodes. To further understand the benefits of the proposed policy, we have compared the obtained results with those attained under other common, intuitive and reasonable transmission policies; a common practice in the literature [19], [24]. It is worth remarking that we employ these policies due to the absence of standardized communication policies for this kind of network in the related literature. These policies are described below:

- Always Store Policy (ASP): The generated events are always stored (as long as there is enough battery to do so) and transmitted when nano-nodes are within the coverage of a nano-router. This policy never willingly drops an event.

- Only Store High-Priority Events Policy (OSHP): Discards low-priority events $\left(e_{1}\right)$, storing and transmitting 


\section{CHAPTER 6. OPTIMAL TRANSMISSION POLICY DERIVATION FOR IONT FGNN

(when possible, i.e. within nano-router coverage and high enough energy level) high-priority $\left(e_{2}\right)$ events.

- High-harvesting Policy (HHP): Always transmits when possible (i.e. under the transmission range of a nanorouter) and sets the storing probability proportional to the battery level of nano-nodes. That is, $P($ store $)=\frac{q}{Q_{M A X}}$ where $P$ indicates the probability of the action.

- Conservative Storing Policy (CSP): Estimates whether enough battery will be available to transmit the even by the time the nano-node reaches the next nano-router. If this estimation is positive, the event will be stored, otherwise it will be discarded. To carry out such an estimation, a conservative approach is taken: nano-node assume $D_{N R}$ to be the longest possible distance between nano-routers (that is, $D_{N R}=D_{N R}^{*}+\delta$ ) and the average harvesting rate, $\overline{q_{h}}$ is used, see Eq. 10. Furthermore, events will always be transmitted whenever the nano-node is under the coverage range of a nano-router.

$$
\overline{q_{h}}=\frac{1}{Q_{M A X}} \sum_{q=0}^{Q_{M A X}} q_{h}(q)
$$

- Neutral Storing Policy (NSP): Similar to CSP, it estimates whether enough energy will be available by the time the nano-node gets to the next nano-router. However, the mean value of $D_{N R}$ is assumed for the computation of the policy (which is equivalent to assuming $\delta=0$ ).

These policies (plus the MDP-based proposed one) are evaluated setting several different scenarios with 20 different seeds to obtain reliable prioritized throughput results. As mentioned in Section IV, both the precision in placing nano-routers an the number of such nano-routers are important variables that are investigated. As previously indicated, we have mathematically defined the distance between nano-routers as $D_{N R}=$ $U\left[D_{N R}^{*}-\delta, D_{N R}^{*}+\delta\right]$. In the first experiment we focus on highlighting the importance of the precision in placing nanorouters. To do so, $D_{N R}^{*}$ is fixed to $5 \mathrm{~cm}$ and $\delta$ is varied from $0 \mathrm{~cm}$ to $3 \mathrm{~cm}$. That is, we assume that our aim is to place a fixed set of nano-routers every $5 \mathrm{~cm}$, but we do so with different degrees of precision $(\delta)$. Values for $D_{N R}^{*}$ and $\delta$ are set in line with the envisaged distances for nano-networks [1] [42]. Again, these values can be tuned by future researchers to be adapted to their applications. Conversely, in the second experiment, $\delta$ is set to $1 \mathrm{~cm}$ and $D_{N R}^{*}$ is varied from $1 \mathrm{~cm}$ to $10 \mathrm{~cm}$. This effectively changes the total number of deploye nano-routers (or the linear density of them) and allows us to assess the impact of such a value on the final performance of nano-nodes.

Finally, regarding the event-generation rate, the three conditions depicted in Table II have been analyzed with increasing values of $\lambda_{1}$ and $\lambda_{2}$.

A. Evaluation of the imprecision in placing nano-routers

Fig. 4 represents the obtained prioritized throughput per nano-node vs. $\delta$ under different generation scenarios and for different transmission policies. The first significant observation is that the proposed MDP-based approach (in blue) systematically outperforms the rest of the policies in every evaluated scenario. While the suitability of a particular alternative policy depends on the scenario, the MDP-based approach effectively adapts its behavior to nano-node conditions. This is noticed by the fact that, for example, the OSHP (in black) is the second best policy in the HGS (see Fig. 4c), whereas it falls to the fourth position in the LGS. Also, as one might expect and will be further analyzed in Subsection V-C, for every policy, when the event-generation rates increase, the throughput also increases as more events are being sent.

Another notable fact is that when $\delta$ increases (that is, the accuracy in placing nano-routers decreases), the MDPbased policy tends to perform worse. This arises fundamentally because Markov Decision Processes statistically estimate the best course of action at each state based on current knowledge (expressed as the state of the nano-node). When this knowledge is imperfect, i.e. we only know the position of the next nano-router with some precision, chosen actions do not always receive the largest rewards. However, note that optimal actions are still those that, on average, produce the largest rewards. It can also be noted that the penalty derived from the lack of knowledge increases as the event-generation rate becomes higher. As can be seen in Fig. 4a, the impact of increasing $\delta$ on the MDP-based policy performance is almost negligible, while progressively raises as the event-generation becomes higher (see Fig. 4b and Fig. 4c).

Conversely, those policies that do not rely on the nanonode position (such as ASP, OSHP, or HHP) are invariant to $\delta$; although they, in fact, tend to perform worse than our proposal. In the case of CSP and NSP (that do depend on $\delta$ ), we highlight that NSP always performs better than CSP thus indicating that considering the expected distance to the next nano-router $\left(D_{N R}=D_{N R}^{*}\right)$ is a better approach than considering the worst-case scenario $\left(D_{N R}=D_{N R}^{*}+\delta\right)$. It is also remarkable how assuming a worst-case scenario strongly degrades the performance when the imprecision in placing nano-routers increases (that is, CSP deteriorates rapidly as increases).

\section{B. Evaluation of the number of deployed nano-routers}

Fig. 5 represents the obtained prioritized throughput per nano-node when the density/number of deployed nano-routers is varied. When the average distance between nano-routers $\left(D_{N R}^{*}\right)$ is lengthened, nano-nodes undergo longer out-ofcoverage periods. This increases the delay between generation and transmission of the event and also makes nano-node batteries deplete faster (as events held in RAM memory require constant energy). These two factors ultimately lead to a reduction in the prioritized throughput (which is appreciated in the Y axis). As in the previously studied case, the MDPbased policy outperforms the rest of the policies; especially for the HGS. Regarding the best alternative policy, it varie with respect to the scenario under consideration. For the LGS and MGS (see Fig. 5a and Fig. 5b), the NSP attains higher values of throughput than the ASP, OSHP, HHP, and CSP. In contrast, for the HGS (see Fig. 5c), where more high-priority events are generated, the best alternative policy is the OSHP as it devotes all the nano-node energy to events of higher priority. 


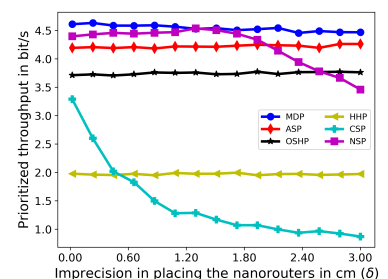

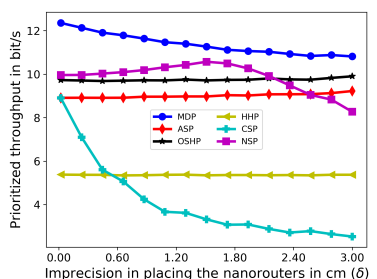

(b)

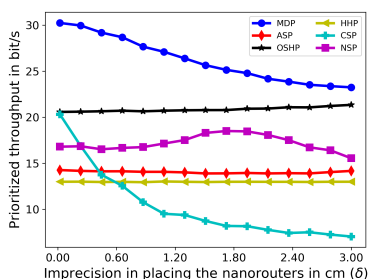

(c)

Fig. 4: Prioritized throughput per nano-node vs. $\delta$ for three event-generation scenarios. Nano-routers are deployed with a distance between them defined as $D_{N R}=U[5 \mathrm{~cm}-\delta, 5 \mathrm{~cm}+\delta]$. (a) Low-Generation Scenario -LGS-, (b) Mid-Generation Scenario -MGS-, and (c) High-Generation Scenario -HGS-.

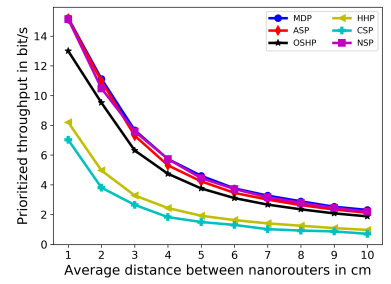

(a)

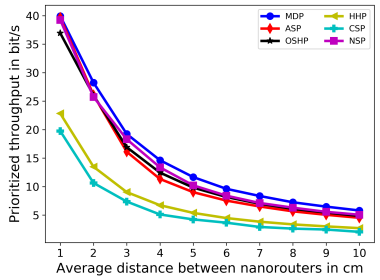

(b)

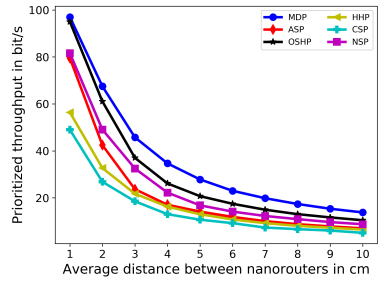

(c)

Fig. 5: Prioritized throughput per nano-node vs. average distance between nano-routers for three event-generation scenarios. Precision in placing nano-routers is set to $\delta=1 \mathrm{~cm}$, thus the distance between them is defined as $D_{N R}=$ $U\left[D_{N R}^{*}-1 \mathrm{~cm}, D_{N R}^{*}+1 \mathrm{~cm}\right]$. (a) Low-Generation Scenario -LGS-, (b) Mid-Generation Scenario -MGS-, and (c) HighGeneration Scenario -HGS-

Moreover, it can be appreciated how, to achieve the same value of throughput, the proposed policy can effectively reduce the number of deployed routers (as the average distance between them, $D_{N R}^{*}$, can be lengthened). For example, analyzing Fig. $5 \mathrm{c}$, it can be seen that to obtain a prioritized throughput of $20 \mathrm{bps}$ in this scenario, the inter-router distance can be relaxe to $7 \mathrm{~cm}$ when the MDP-based policy is used. This represents a reduction of $34.6 \%$ in the number of deployed nano-router with respect to the best alternative policy (OSHP), which requires an inter-router distance of $5.2 \mathrm{~cm}$. When the rest of the policies are employed, this reduction increases up to $57.6 \%$ (NSP), $97.1 \%$ (ASP), $112.1 \%$ (HHP), and $150 \%$ (CSP).

Cutting back on the number of nano-routers to be deployed has many advantages. Among them, we should remark on the reduction of the cost of nano-networks, easing their maintenance and potentially making their deployment less invasive (in the case of nano-networks installed within humans).

\section{Evaluation of the event-generation rate}

Fig. 6 represents the obtained prioritized throughput per nano-node when the probability of generating events (that is, $\lambda_{1}+\lambda_{2}$ ) is varied from $1 \%$ to $20 \%$. The distance between nanorouters is set to $D_{N R}=5 \mathrm{~cm}$ and the imprecision to $\delta=0$.
As observed in previous experiments, the degree to which the MDP-based policy outperforms the second best policy tightly depends on the total event-generation probability. Precisely, what we note is that the higher the traffic generation, the more important smart policies are. This stems from the fact that when very few events are generated, the energy-harvesting rate is large enough to cope with the event-generation rate, and thus, policies that store (and latter transmit) every generated event (such as ASP) tend to perform relatively well. When energy becomes a scarce resource (either because the energyharvesting rate is reduced or the number of generated events increases, as is our case), smart policies such as the proposed one gain in importance. Thus, the MDP-based policy shows a better and more efficient use of the available nano-node resources. Numerically, this can be corroborated by analyzing the performance values obtained for $\lambda_{1}+\lambda_{2}=20 \%$, where the prioritized throughput increases by $97.1 \%$ when our policy is preferred over the second-best policy.

\section{CONCLUSIONS}

The Internet of Nano-Things is a valuable field of study that can pave the way for multiple and promising applications such as in vivo monitoring of the human cardiovascular 


\section{CHAPTER 6. OPTIMAL TRANSMISSION POLICY DERIVATION FOR IONT FGNN

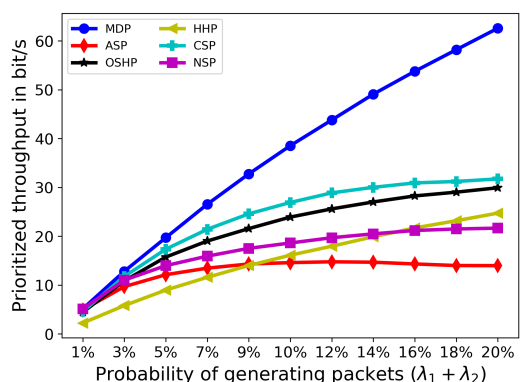

Fig. 6: Prioritized throughput per nano-node vs. the probability of a node generating an event $\left(\lambda_{1}+\lambda_{2}\right)$. The generation ratio between low-priority $\left(\lambda_{1}\right)$ and high-priority $\left(\lambda_{2}\right)$ events has not been modified $\left(\lambda_{1}=2 \cdot \lambda_{2}\right)$.

system. However, and as explored in this work, this particular kind of environment, coined as flow-guided environment, is not trouble-free. Nano-devices undergo long out-of-coverage periods and must carefully manage the harvested energy to promptly and effectively report sensed events. To deal with these two problems, and at the same time, to maximize network throughput, we have proposed a general yet powerfu mathematical framework that can assist designers in deriving smart transmission policies.

To guide researchers through the application of the presented model, we have particularized it to the case of nanonetworks deployed within the cardiovascular system. Along with such a particularization, an extensive set of simulation are carried out to (i) validate the mathematical MDP mode and (ii) demonstrate the superior performance of the propose model when compared to other reasonable alternative transmission policies. By analyzing a wide variety of potential scenarios, we have shown that our method effectively adapts the proposed transmission policies to the particular characteristics of each environment, adjusting the behavior of nanonodes and optimizing their performance. Interestingly, we have also analyzed the impact on network performance of two key factors in flow-guided environments: the number of and separation between nano-routers. Results reveal that while nanonodes benefit a from denser, precisely-placed nano-network infrastructure, the proposed policy can perform on par with other alternative policies with $34.6 \%$ to $150 \%$ fewer routers. By decreasing the number of deployed nano-routers, we not only reduce the cost of nano-networks but also make their implantation less invasive; ultimately facilitating the adoption of this promising technology and making it more achievable in the near future.

\section{ACKNOWLEDGMENT}

This work has been supported by the project AIM, ref. TEC2016-76465-C2-1-R (AEI/FEDER, UE). Sebastian Canovas-Carrasco and Ruben M. Sandoval also thank the
Spanish MECD for an FPU (refs. FPU16/03530 and FPU14/03424) pre-doctoral fellowship.

\section{REFERENCES}

11] I. Akyildiz and J. Jornet, "The Internet of nano-things," IEEE Wireless Communications, vol. 17, no. 6, pp. 58-63, Dec 2010 .

2] M. Stelzner, F. Dressler, and S. Fischer, "Function Centric NanoNetworking: Addressing nano machines in a medical application scenario," Nano Communication Networks, vol. 14, pp. 29-39, 2017.

3] I. F. Akyildiz and J. M. Jornet, "Electromagnetic wireless nanosensor network."
2010 .

4] G. Piro, P. Bia, G. Boggia, D. Caratelli, L. Grieco, and L. Mescia, "Terahertz electromagnetic field propagation in human tissues: A study on
communication capabilities," Nano Communication Networks, vol. 10, no. December 2016, pp. 51-59, Dec. 2016.

[5] Y. Chahibi, "Molecular communication for drug delivery systems: A survey," Nano Communication Networks, vol. 11, pp. 90-102, Mar. 2017 .

[6] L. Felicetti, M. Femminella, G. Reali, and P. Liò, "Applications of molecular communications to medicine: A survey," Nano Communication Networks, vol. 7, pp. 27-45, Mar. 2016.

[7] P. M. Kosaka, V. Pini, J. J. Ruz, R. a. da Silva, M. U. González D. Ramos, M. Calleja, and J. Tamayo, "Detection of cancer biomarkers in serum using a hybrid mechanical and optoplasmonic nanosensor," Nature Nanotechnology, vol. 9, no. 12, pp. 1047-1053, Nov. 2014.

[8] S. Canovas-Carrasco, A.-J. Garcia-Sanchez, and J. Garcia-Haro, "The IEEE 1906.1 Standard: Some Guidelines for Strengthening Future Normalization in Electromagnetic Nanocommunications," IEEE Communications Standards Magazine, vol. 2, no. 4, pp. 26-32, Dec. 2018

[9] K. Yang, A. Pellegrini, M. O. Munoz, A. Brizzi, A. Alomainy, and Y. Hao, "Numerical Analysis and Characterization of THz Propagation Channel for Body-Centric Nano-Communications, IEEE Transactions on Terahertz Science and Technology, vol. 5, no. 3, pp. 419-426, May

[10] S. Canovas-Carrasco, A.-J. Garcia-Sanchez, and J. Garcia-haro, "A nanoscale communication network scheme and energy model for a human hand scenario,"

1] S. M. Demir, F. Al-Turjman, and A. Muhtaroglu, "Energy Scavenging Methods for WBAN Applications: A Review," IEEE Sensors Journal, vol. 18, no. 16, pp. 6477-6488, Aug. 2018.

[12] J. M. Jornet and I. F. Akyildiz, "Joint Energy Harvesting and Communication Analysis for Perpetual Wireless Nanosensor Networks in the pp. 570-580, May 2012 .

[13] S. Canovas-Carrasco, A.-J. Garcia-Sanchez, F. Garcia-Sanchez, and J. Garcia-Haro, "Conceptual Design of a Nano-Networking Device," Sensors, vol. 16 , no. 12 , p. 2104 , Dec. 2016

[14] P. Singh, B.-W. Kim, and S.-Y. Jung, "TH-PPM with non-coherent detection for multiple access in electromagnetic wireless nacommuncations," Nano Communication Networks, vol. 17, pp. 1-13, Sep. 2018 [15] F. Al-Turjman, "A rational data delivery framework for disaster-inspired
internet of nano-things (IoNT) in practice," Cluster Computing, Nov. 2017

16] F. Afsana, M. Asif-Ur-Rahman, M. R. Ahmed, M. Mahmud, and M. S. Kaiser, "An Energy Conserving Routing Scheme for Wireless Body Sensor Nanonetwork Communication," IEEE Access, vol. 6, pp. 91869200, 2018.

17] H. Yu, B. Ng, and W. K. G. Seah, "TTL-based Efficient Forwarding for Nanonetworks with Multiple Coordinated IoT Gateways," IEEE Intern of Things Journal, vol. 5, no. 3, pp. 1807-1815, Jun. 2018.

18] N. Akkari, P. Wang, J. M. Jornet, E. Fadel, L. Elrefaei, M. G. A. Malik S. Almasri, and I. F. Akyildiz, "Distributed Timely Throughput Optima Scheduling for the Internet of Nano-Things," ILEE Internet of Things

[19] R. M. Sandoval, A.-J. Garcia-Sanchez, J. Garcia-Haro, and T. M. Chen "Optimal Policy Derivation for Transmission Duty-Cycle Constrained 3125, Aug. 2018.

20] O. Ozel, K. Tutuncuoglu, J. Yang, S. Ulukus, and A. Yener, "Transmission with energy harvesting nodes in fading wireless channels: Optimal . no. 8, pp. 1732-1743, Sept. 2011. 


\section{CHAPTER 6. OPTIMAL TRANSMISSION POLICY DERIVATION FOR}

[21] C. Tunc and N. Akar, "Markov fluid queue model of an energy Tevice with adative sensing." Performance Evaluation, vol. 111, pp. 1-16, May 2017

[22] W. Zhu, P. Xu, M. Zheng, G. Wu, and H. Wang, "Transmission policies for energy harvesting sensors based on markov chain energy supply," Networks, 2015,

[23] R. M. Sandoval, S. Canovas-Carrasco, A. Garcia-Sanchez, and J. GarciaHaro, "Smart Usage of Multiple RAT in IoT-Oriented 5G Networks: A Reinforcement Learning Approach," in 2018 ITU Kaleidoscope: Machine Learning for a 5 G Future (ITU K), Nov. 2018, pp. 1-8.

[24] S. Mohrehkesh and M. C. Weigle, "Optimizing Energy Consumption in Terahertz Band Nanonetworks," IEEE Journal on Selected Areas in Communications, vol. 32, no. 12, pp. 2432-2441, Dec. 2014.

[25] M. A. Alsheikh, D. T. Hoang, D. Niyato, H.P. Tan, and S. Lin, "Markov Decision Processes With Applications in Wireless Sensor Networks: Survey," IEEE Communications Surveys Tutorials, vol. 17, no. 3, pp. 1239-1267, Apr. 2015.

[26] R. S. Sutton and A. G. Barto, Introduction to Reinforcement Learning 1st ed. Cambridge, MA, USA: MIT Press, 1998.

[27] N. Michelusi, K. Stamatiou, and M. Zorzi, "Transmission policies for energy harvesting sensors with time-correlated energy supply," IEEE Transactions on Communications, vol. 61, no. 7, pp. 2988-3001, Ju

8] M. Wiering and M. Van Otterlo, "Reinforcement learning," Adaptation, Learning, and Optimization, vol. 12, Jan. 2012.

Drundage, and A. A. Bharath, "A Brief Survey of Deep Reinforcement Learning," arXiv e-prints, p.

arXiv:1708.05866, Aug. 2017.
30] Q. H. Abbasi, H. El Sallabi, N. Chopra, K. Yang, K. A. Qaraqe, and for nano-scale body-centric networks," Science and Technology, vol. 6, no. 3, pp. 427-434, May 2016.

[31] P. Johari and J. M. Jornet, "Nanoscale Optical Wireless Channel Model for Intra-Body Communications: Geometrical, Time, and Frequency
Domain Analyses," IEEE Transactions on Communications, vol. 66, no. 4, pp. 1579-1593, Apr. 2018

[32] Q. H. Abbasi, A. A. Nasir, K. Yang, K. A. Qaraqe, and A. Alomainy, "Cooperative In-Vivo Nano-Network Communication at Terahertz Frequencies," IEEE Access, vol. 5, pp. 8642-8647, 2017

[33] R. M. Sandoval and S. Canovas-Carrasco, "Pytho scripts for simulations," 2019. [Online]. Available:

https://github.com/rubmarsan/IoTjournal-IEEE-2019
[34] F. Al-Turjman, M. Imran, and A. Vasilakos, "Value-Based Caching in Information-Centric Wireless Body Area Networks," Sensors, vol. 17,

35] H. Yu, B. Ng, and W. K. Seah, "On-Demand Probabilistic Polling for Nanonetworks under Dynamic IoT Backhaul Network Conditions,

6] M. Klarhofer, B. Csapo, C. Balassy, J. Szeles, and E. Moser, "HighMagnetic Resonance in Medicine, vol. 45, no 4, pp. 716-719, Aps. 2001

[37] H. Elayan, R. M. Shubair, J. M. Jornet, and P. Johari, "Terahertz Channel Model and Link Budget Analysis for Intrabody Nanoscale Communication," IEEE Transactions on NanoBioscience, vol. 16, no. 6, pp. 491-503, Sep. 2017.

[38] J. M. Jornet and I. F. Akyildiz, "Femtosecond-Long Pulse-Based Modulation for Terahertz Band Communication in Nanonetworks," IEEE Transactions on Communications, vol. 62, no. 5, pp. 1742-1754, May 2014

[39] S. Canovas-Carrasco, A.-J. Garcia-Sanchez, and J. Garcia-Haro, "O the Nature of Energy-Feasible Wireless Nanosensor Networks," Sensor. vol. 18 , no. 5, p. 1356, Apr. 2018.

[40] M. H. Ghaed, G. Chen, R.-u. Haque, M. Wieckowski, Y. Kim, G. Kim Y. Lee, I. Lee, D. Fick, D. Kim, M. Seok, K. D. Wise, D. Blaauw, and D. Sylvester, "Circuits for a Cubic-Millimeter Energy-Autonomous and Systems I: Regular Papers, vol. 60, no. 12, pp. 3152-3162, Dec. and Systems I: Regular Papers, vol. 60, no. 12, pp. 3152-3162, Dec.

[41] Simpy, "Event discrete simulation for Python." [Online]. Available:

https://simpy.readthedocs.io
[42] J. M. Jornet and I. F. Akyildiz, "The internet of multimedia NanoThings" Namo Communication Networks, vol. 3, no. 4, pp. 242-251, Dec. 2012 
Chapter 7

An Analytical Approach to
Flow-Guided
Nanocommunication Networks 


\title{
Article
}

\section{An Analytical Approach to Flow-Guided Nanocommunication Networks}

\author{
Rafael Asorey-Cacheda ${ }^{*}, \boldsymbol{+}^{+}$, Sebastian Canovas-Carrasco ${ }^{+}+{ }^{\circ}$, Antonio-Javier Garcia-Sanchez ${ }^{+}(\mathbb{C}$ \\ and Joan Garcia-Haro ${ }^{+}(\mathbb{B}$ \\ Department of Information and Communication Technologies, Universidad Politécnica de Cartagena, \\ 30202 Cartagena, Spain; sebas. canovas@upct.es (S.C.-C.); antoniojavier.garcia@upct.es (A.-J.G.-S.); \\ joang.haro@upct.es (J.G.-H.) \\ * Correspondence: rafael.asorey@upct.es; Tel.: +34-968-326-537 \\ + These authors contributed equally to this work. \\ Received: 30 January 2020; Accepted: 25 February 2020; Published: 29 February 2020

Abstract: Continuous progress of nanocommunications and nano-networking is opening the door to the development of innovative yet unimaginable services, with a special focus on medical applications. Among several nano-network topologies, flow-guided nanocommunication networks have recently emerged as a promising solution to monitoring, gathering information, and data communication inside the human body. In particular, flow-guided nano-networks display a number of specific characteristics, such as the type of nodes comprising the network or the ability of a nano-node to transmit successfully, which significantly differentiates them from other types of networks, both at the nano and larger scales. This paper presents the first analytical study on the behavior of these networks, with the objective of evaluating their metrics mathematically. To this end, a theoretical framework of the flow-guided nano-networks is developed and an analytical model derived. The main results reveal that, due to frame collisions, there is an optimal number of nano-nodes for any flow-guided network, which, as a consequence, limits the maximum achievable throughput. Finally, the analytical results obtained are validated through simulations and are further discussed.

Keywords: flow-guided nano-networks; analytical model; nanocommunications

\section{Introduction}

Recent advances in nanotechnology are laying the foundations for the development of novel medical applications that can significantly improve the effectiveness of disease diagnosis and treatment [1-5]. One of the most promising techniques is based on the deployment of size-constrained devices endowed with a wireless communication module, known as nano-nodes, inside the human body, where larger devices would be inappropriate or excessively invasive. These nano-nodes, measured in micrometers, could be injected into the bloodstream providing real time in-vivo measurements of different elements present in the blood. For example, each nano-node could be equipped with a nano-sensor similar to that proposed in [6], enabling the detection of cancer biomarkers at an early stage. This type of nano-network, in which nano-nodes continuously move within a well-defined flow, is known as a flow-guided nanocommunication network [7].

Flow-guided nanocommunication networks pose two important challenges that must be addressed. On the one hand, as the miniaturization of the radiating antenna integrated into each nano-node demands the use of high frequencies to communicate (around $1 \mathrm{THz}[2,8]$ ), the attenuation of electromagnetic signals within the human body (largely made up of water) is very high $[9,10]$ Inherently, this high path loss severely limits the communication range, making direct communication between nano-nodes and a macro device (e.g., a wearable device or a smartphone) impractical [3,11] 
On the other hand, the extremely limited amount of energy that can be stored in a nano-node (due to technological constraints [12]) requires the use of a piezoelectric nano-generator to recharge the battery periodically $[13,14]$. Therefore, the energy balance in flow-guided nano-networks must be examined considering both the energy consumed and the energy harvested from the environment

To alleviate these two shortcomings caused by the extremely reduced size of nano-nodes, larger and less resource-constrained devices called nano-routers act as a middle network tier that gathers all the raw data generated by nano-nodes, and then sends them to a macro device, which can connect the nano-network to the Internet [15-18]. Even though nano-routers are larger in size than nano-nodes, they are envisaged to be small enough to be implanted in the human body. Thus, through strategic placement, the distance of the communication link between the nano-node and nano-router can be minimized to ensure that nano-nodes are able to transmit the generated data, employing small amounts of available energy [11]. Thereby, nano-nodes will successfully transmit a data frame when they pass through the coverage range of a nano-router. As nano-nodes must keep energy consumption as low as possible, we consider that a nano-node ignores whether it is in the coverage area or not; i.e., no energy is consumed listening to the channel.

As the characteristics of flow-guided nano-networks are quite different to other nano-network topologies, a theoretical framework capturing all their peculiarities is needed to properly model their behavior and set theoretical boundaries on their performance. For this purpose, in this paper, we propose a general analytical model to mathematically evaluate different metrics of flow-guided nano-networks. We then derive closed-form equations for the maximum achievable throughput as a function of the number of nano-nodes in the network, the coverage range of the nano-routers, the battery recharge frequency, the average nano-node velocity, and the number of transmission slots. We also employ these equations to analyze the impact on the throughput of each one of these parameters. To summarize, the main contributions of this work are as follows:

- We develop an analytical model capturing all the particularities of flow-guided networks. We note that this model is general enough to be implemented in different applications in which flow-guided networks could be used. To the best of our knowledge, no previous mathematical model for this type of networks exists.

- We provide insightful results about the achievable throughput of flow-guided nano-networks as a function of different important parameters.

- We calculate the maximum number of nano-nodes that can compose a flow-guided nano-network without penalizing the total throughput of the network. It should be noted that, when the number of nano-node is excessively high, data frame collisions significantly hinder the throughput achieved.

- We assume a time-slotted transmission technique to increase the achievable throughput of flow-guided nano-networks. Results obtained show that this slotted technique substantially increases the total capacity of the network.

- We validate the proposed mathematical framework by simulating a flow-guided nano-network under different scenarios. The results obtained with the simulations have been compared to those attained by the analytical equations, showing that the mathematical framework accurately models the behavior of a flow-guided nano-network.

The rest of the paper is organized as follows. Section 2 outlines all the related work. Section 3 rigorously defines the analytical model. All the results are discussed in Section 4, including those obtained from simulations. The impact on the network throughput of each model parameter is discussed, highlighting the benefits of employing a slotted transmission scheme and the effect of frame length on overall performance. Finally, Section 5 concludes the paper. Appendix A further discusses the behavior of the simultaneous number of collisions in flow-guided nano-networks. 


\section{Related Work}

Since nano-networks are still in their infancy, many works in the literature are devoted to theoretically modeling the behavior of different types of nano-networks. Authors in [19] proposed a routing framework to maximize the overall network throughput of a hierarchical nano-network. The network architecture studied in that work consisted of static nano-sensors and nano-controllers, in which nano-sensors were divided into clusters, each one associated to a nano-controller. In contrast, our work considers mobile nano-nodes and static nano-routers. In [3], a theoretical study of the communication capabilities of a nano-network deployed inside a human body is developed. By thoroughly taking into account the dielectric properties of different biological tissues, the maximum channel capacity as a function of the transmission distance for different communication schemes is obtained. Results suggest that, since the transmission distances are limited to obtain an acceptable capacity, the development of nano-networks in the human body requires multi-hop network topologies (as proposed in our work).

In [20], the maximum achievable throughput of a nano-network composed of $n$ randomly distributed identical nodes is analyzed. The main characteristics of an electromagnetic nano-network are adequately considered and captured in the theoretical model. Results reveal that, for high-density nano-networks (over $10^{7}$ nodes per unit area), interferences between nano-nodes limit the achievable throughput. Similarly, Section 3 of our work determines the maximum achievable throughput and the number of nano-nodes necessary to obtain it particularized to flow-guided nano-networks.

Regarding the study of flow-guided nano-networks, the work in [11] proposed a realistic flow-guided nano-network, following a hierarchical topology based on three network layers: nano-nodes, a nano-router, and an Internet gateway. It was designed to monitor the human body with the nano-nodes injected into the bloodstream communicating with a nano-router strategically implanted in the skin. Nano-nodes transmit the generated data to this static nano-router when they are in its vicinity. In turn, the nano-router sends the received data to an Internet gateway placed outside the body, connecting the nano-network to the Internet. However, the main limitation of this flow-guided nano-network is that only one nano-router is considered, severely restricting the nano-network capacity. It should be noted that this work proposes a deterministic and realistic case of using flow-guided nano-networks. Conversely, our present work provides a general mathematical model to analyze the different performance metrics of the network.

Finally, the authors in [7] proposed a mathematical framework to derive optimal transmission policies for flow-guided nano-networks. Results indicated that intelligently using the limited resources of nano-nodes, the network throughput can be increased in a realistic case of study (based on the human circulatory system). This work laid the foundations to formulate and derive our analytical model as described in the following sections.

\section{Analytical Model}

\subsection{Model Description}

The analytical model is based on the following assumptions:

- $\quad$ There are $n$ nano-nodes uniformly distributed along the flow, $n \geq 1, n \in \mathcal{N}$.

- The flow network is a closed circuit in which the nano-nodes continuously circulate. An example of a flow network might be the blood circulatory system of the human body.

- A nano-node moves within the flow at average speed $v$ and requires $T$ time units to complete a round.

- A nano-node battery is charged every $1 / f$ time units. Moreover, due to energy constraints, a nano-node can only transmit one data frame per battery charge. The work in [14] provides more details on a realistic model based on piezo-electric elements to charge a nano-node battery. In this 
paper, we assume a similar battery charging mechanism. Thus, a nano-node can only harvest enough energy to transmit a single frame every $1 / f$ time units.

- A successful transmission can only occur when a nano-node is close enough to a nano-router and no other nano-node transmits at the same time (see Figure 1). For the sake of simplicity, without a loss of generality, let us assume that there is a region in the flow of length $A$, in which a nano-node frame transmission can successfully reach a network nano-router (a nano-node is under the nano-router coverage zone). Thus, the probability of a nano-node being in the coverage zone $\left(p_{A}\right)$ can be modeled as

$$
p_{A}=\frac{A}{v T}
$$

For instance, nano-router coverage is a volume that could be a section of a vein or an artery; its characterization as the length of magnitude $A$ does not imply a loss of generality. Let us assume that the flow network is cylindrically shaped and denote $r$ as its radius. Thus, the volume of the area of coverage is $A \pi r^{2}$, and the total network volume is $v T \pi r^{2}$. As a consequence, $A \pi r^{2} /\left(v T \pi r^{2}\right)=A /(v T)=p_{A}$. The nano-node coverage model of this paper is based on [11]. It can be consulted for more details on nano-node coverage issues in flow guided nano-networks.

- Let us assume that $A / v<1 / f$, i.e., the time between transmissions is longer than the time used by a nano-node to cross the coverage area. In other words, a nano-node cannot perform more than one transmission when crossing the coverage area.

- A nano-node ignores if it is within the coverage area due to its energy limitations. Thus, any nano-node will try to perform a successful transmission at every battery charge cycle, independently of its position in the flow-guided network. These transmissions can occur at any moment between two consecutive battery charges.

- The model described in this paper assumes that the time between two battery charges, $1 / f$, is divided into $\sigma$ slots $(\sigma \geq 1, \sigma \in \mathcal{N})$ and a nano-node transmits a frame randomly, with a probability $1 / \sigma$, in one of these slots. The maximum number of slots is limited by the frame size. Thus, if $t_{f}$ is the time required to transmit a frame, $t_{f} \leq(f \sigma)^{-1}$.

- A successful frame transmission takes place if it starts and ends within the coverage area and no collision occurs. As a consequence, the transmission zone, $A_{t x}$, is smaller than the coverage area, $A_{t x}=A-v t_{f}$, as any successful transmission must start and end within the coverage area (see Figure 1). Thus, note that the probability of a nano-node being in the transmission zone is as follows:

$$
p_{t x}=\frac{A-v t_{f}}{v T}, v t_{f}<A
$$

- A collision occurs if one or more transmissions start or end within the coverage zone while another transmission in the transmission zone takes place. As a consequence, the collision zone, $A_{c x}$ is larger than the coverage area, $A_{c x}=A+v t_{f}$, as a collision can only be avoided if it starts and ends outside the coverage area (see Figure 1). Thus, the probability of a nano-node being in the collision zone is as follows:

$$
p_{c x}=\frac{A+v t_{f}}{v T}, v t_{f}<A
$$

- $\quad$ For the sake of simplicity, in many cases, it can be assumed that $v t_{f} \lll A$, which is a realistic assumption according to the values used in [7]. Thus, a frame collision event can only be considered when two or more nodes transmit within the coverage area of the nano-router in the same transmission slot. As a consequence, transmissions starting outside the coverage area and ending inside, or vice versa, can be neglected. If $v t_{f} \lll A$ holds, the following approximation can be made:

$$
p_{t x} \approx p_{c x} \approx p_{A}, v t_{f} \lll A
$$


- This model assumes that $A \ll v T$, as in [7], which is a realistic assumption in flow-guided nano-networks.

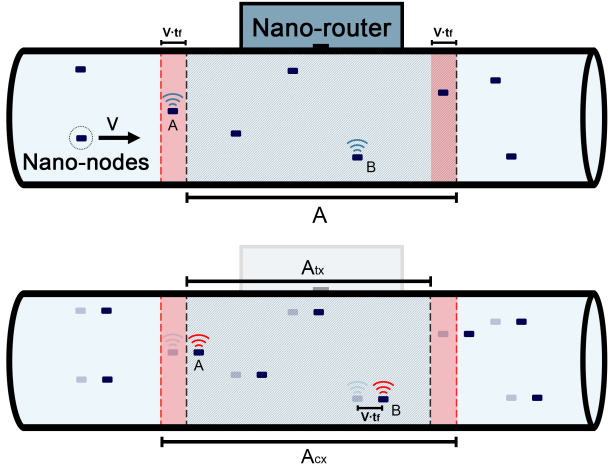

Figure 1. Scenario in which transmissions from Nano-Nodes A and B collide. The upper part of the figure represents the initial state at $t_{0}$ (Nano-Node $\mathrm{A}$ within the transmission zone and Nano-Node $\mathrm{B}$ within the collision zone; i.e., transmissions do not collide yet). The lower part of the figure represents the final state after the transmissions end at $t_{0}+t_{f}$ (Nano-Nodes A and B within the transmission zone; i.e., a collision occurs)

Table 1 specifies all the variables used in this analytical model.

Table 1. Variables used in the analytical model and their description.

\begin{tabular}{ll}
\hline Variable & Description \\
\hline$n$ & $\begin{array}{l}\text { The number of nano-nodes, uniformly distributed, in the flow-guided nano-network, } n \geq 1, \\
n \in \mathcal{N} .\end{array}$ \\
$v$ & Average speed of the nano-node in the flow in $\mathrm{m} / \mathrm{s}$. \\
$T$ & Time required by a nano-node to complete a round in the flow-guided network in s. \\
$f$ & Nano-node charging frequency in $\mathrm{Hz}$. \\
$A$ & Length of the coverage area in $\mathrm{m}$. \\
$A_{t x}$ & Length of the transmission zone in $\mathrm{m}, A_{t x}=A-v t_{f}$. \\
$A_{c x}$ & Length of the collision zone in $\mathrm{m}, A_{c x}=A+v t_{f}$. \\
$\sigma$ & Number of transmission slots between two battery charges, $\sigma \geq 1, \sigma \in \mathcal{N}$. \\
$t_{f}$ & Time required to transmit a frame in s. \\
\hline
\end{tabular}

3.2. Achievable throughput

As a first approach, let us assume that $A \gg v t_{f}$ and $p_{t x} \approx p_{c x} \approx p_{A}$; i.e., frame length can be neglected. At the end of this section, throughput equations will be generalized for arbitrary lengths of $t_{f}$.

According to the the model of Section 3.1, it is possible to obtain an analytical equation of the achievable network throughput. Thus, a successful transmission happens when a nano-node transmits a frame within a nano-router coverage area and no other nano-node is in the coverage area or, if it is within, it does not transmit in the same time slot.

As nano-nodes are uniformly distributed along the flow network, the probability of transmitting within the coverage area depends on (i) $p_{A}$ and (ii) the probability of performing a transmission while crossing the coverage area. The probability of a nano-node being outside of the coverage area can be 
derived from Equation (1) as $1-p_{A}$. Moreover, the probability of another nano-node being in the coverage area and not transmitting in a given time slot, $p_{A, n t x}$, can be modeled as follows:

$$
p_{A, n t x}=p_{A}\left(1-\frac{1}{\sigma}\right)=\frac{A}{v T}\left(1-\frac{1}{\sigma}\right)
$$

The successful transmission probability to a nano-router for a node, $p_{A, t x}$, can be formulated as follows:

$$
p_{A, t x}=p_{A} \frac{1}{\sigma} \sum_{k=0}^{n-1}\left(\begin{array}{c}
n-1 \\
k
\end{array}\right)\left(1-p_{A}\right)^{n-1-k} p_{A, n t x}^{k}
$$

Equation (6) represents the probability of a nano-node within the coverage area transmitting a frame without collisions. The summation of Equation (6) can be simplified as follows:

$$
p_{A, t x}=\frac{p_{A}}{\sigma}\left(1-\frac{p_{A}}{\sigma}\right)^{n-1}=\frac{A}{v T \sigma}\left(1-\frac{A}{v T \sigma}\right)^{n-1}
$$

From Equation (7), the network throughput can be derived as $\operatorname{Th}(n, A, \sigma)=n f \sigma p_{A, t x}$, corresponding to $n$ nano-nodes and the the event transmission rate, $f \sigma$ :

$$
\operatorname{Th}(n, A, \sigma)=\frac{n A f}{v T}\left(1-\frac{A}{v T \sigma}\right)^{n-1}
$$

Equation (8) shows that network throughput increases with the number of slots, as can be expected, as well as for a moderate number of nano-nodes in the network. However, if the number of nano-nodes is too large, throughput converges to 0.

3.2.1. Maximum Achievable throughput

The number of nano-nodes, $n_{t h}$, maximizing Equation (8), can be easily obtained from $\operatorname{dTh}(n, A, \sigma) / \mathrm{d} n=0$ :

$$
\frac{\mathrm{dTh}(n, A, \sigma)}{\mathrm{d} n}=\frac{A f}{v T}\left(1-\frac{A}{v T \sigma}\right)^{n-1}\left(1+n \log \left(1-\frac{A}{v T \sigma}\right)\right)=0
$$

Thus, $n_{t h, \sigma}$ is expressed as follows:

$$
n_{t h, \sigma}=-\log \left(1-\frac{A}{v T \sigma}\right)^{-1}
$$

This model assumes that $A \ll v T \sigma$, and Equation (10) can therefore be approximated by the following:

$$
n_{t h, \sigma} \approx \frac{v T \sigma}{A}, A \ll v T \sigma
$$

As can be observed from Equations (10) and (11), the number of nano-nodes required to achieve maximum throughput grows with the number of slots. This can be seen as a drawback, since maintaining the number of nano-nodes as low as possible might be a desired design goal. However, increasing the number of slots also raises throughput for any value of $n$, as demonstrated in Section 3.2.2. Note that, although slotted transmission mechanisms require synchronization that cannot be straightforward to implement, flow-guided nano-network synchronism is externally and naturally provided to nano-nodes by the battery charging cycles. 
The maximum achievable throughput as a function of $A$ and $\sigma$ will be as follows:

$$
\operatorname{Th}_{n}(A, \sigma)=\frac{-A f}{v T \log \left(1-\frac{A}{v T \sigma}\right)}\left(1-\frac{A}{v T \sigma}\right)^{-\log \left(1-\frac{A}{v T \sigma}\right)^{-1}-1}
$$

Equation (12) can be simplified as follows ( $e$ is Euler's number):

$$
\operatorname{Th}_{n}(A, \sigma)=\frac{-A f}{e v T \log \left(1-\frac{A}{v T \sigma}\right)\left(1-\frac{A}{v T \sigma}\right)}
$$

Since in this model it is assumed that $A \ll v T \sigma$, Equation (13) can be further approximated by the following:

$$
\operatorname{Th}_{n}(\sigma) \approx \frac{f \sigma}{e}, A \ll v T \sigma
$$

As a consequence, the maximum achievable throughput does not rely on $A$, but on the number of slots, $\sigma$

3.2.2. Benefits of Slot Utilization

The positive impact of using slots on the achievable throughput can be quantified as $\eta_{t h}(A, \sigma)=$ $\mathrm{Th}_{n}(A, \sigma) / \mathrm{Th}_{n}(A, 1)$. Using Equation (14),

$$
\eta_{t h}(A, \sigma) \approx \sigma, A \ll v T \sigma
$$

However, this improvement can only be attained by increasing the number of nano-nodes in the flow-guided network. Let us define $\eta_{n}(A, \sigma)=n_{t h, \sigma} / n_{t h, 1}$. Thus,

$$
\eta_{n}(A, \sigma)=\frac{\log \left(1-\frac{A}{v T}\right)}{\log \left(1-\frac{A}{v T \sigma}\right)}
$$

Assuming that $A \ll v T \sigma$, Equation (16) can be approximated by the following:

$$
\eta_{n}(A, \sigma) \approx \sigma, A \ll v T \sigma
$$

Equations (15) and (17) show that achieving any arbitrary improvement in throughput requires increasing the number of slots and therefore the number of nano-nodes proportionally. Let us define $\eta_{s}(n, A, \sigma)$ as the improvement in throughput for an arbitrary number of slots, but with the same number of nano-nodes, $\eta_{s}(n, A, \sigma)=\operatorname{Th}(n, A, \sigma) / \operatorname{Th}(n, A, 1)$ :

$$
\eta_{s}(n, A, \sigma)=\left(\frac{v T-A / \sigma}{v T-A}\right)^{n-1}
$$

If $\sigma$ is large enough, $\sigma \rightarrow \infty$, Equation (18) can be approximated by the following:

$$
\eta_{s}(n, A, \sigma) \approx\left(\frac{v T}{v T-A}\right)^{n-1}, \sigma \rightarrow \infty
$$

Moreover, in this model, $A \ll v T$ allows us to further approximate Equation (19) as follows:

$$
\eta_{s}(n, A, \sigma) \approx 1+\frac{(n-1) A}{v T}, \sigma \rightarrow \infty, A \ll v T
$$


Equation (19) shows that throughput improvement is bounded and depends on the number of nano-nodes. Moreover, Equation (20) imposes that $n \sim v T / A$, in order for $\eta_{s}(n, A, \sigma)$ to be a significant value. For most scenarios, as will be discussed below in Section 4, this can be difficult to satisfy, and $\eta_{s}(n, A, \sigma) \approx 1$; i.e., if the number of nano-nodes remains constant, increasing the number of slots does not provide a substantial improvement in throughput.

\subsubsection{Length of the Coverage Area}

Since the length $A$ modeling the coverage area is relevant for the values of $p_{A}, p_{t x}$, and $p_{n t x}$ respectively; it can be argued that there is a value of $A$ that maximizes throughput as a function of $n$ and $\sigma$. However, note that the value of $A$ is constrained by an upper limit, $A_{\text {max }}$, derived from the maximum transmission power of the nano-node. On the other hand, a nano-node can reduce its transmission power below its maximum and, consequently, reduce the length $A$ of the coverage area:

- A larger value of $A$ increases the probability of transmitting in the coverage area, $p_{t x}$, as well as the probability of additional nodes in this area, $p_{A}$, and the probability of frame collision, $p_{n t x}$.

- $\quad$ A lower value of $A$ reduces $p_{t x}$, as well as $p_{A}$ and $p_{n t x}$.

An optimal value of $A$ can be obtained from $\operatorname{dTh}(n, A, \sigma) / \mathrm{d} A=0$ :

$$
\frac{\mathrm{dTh}(n, A, \sigma)}{\mathrm{d} A}=\frac{n f}{v T}\left(1-\frac{A}{v T \sigma}\right)^{n-2}\left(1-\frac{n A}{v T \sigma}\right)=0
$$

Equation (21) has two solutions. The first one is $A=v T \sigma$, which is only valid if $\sigma=1$ because $A \leq v T$. The second solution is $A=v T \sigma / n$, which requires $n \geq \sigma$ to be valid. In general, the value of $A$ that maximizes throughput is as follows:

$$
A_{t h}=\min \left\{A_{\max }, \frac{v T \sigma}{n}\right\}
$$

Equation (22) evidences that increasing the number of nodes and decreasing the length of the coverage area leads to a higher network throughput. Moreover, from Equation (22), one can also infer that, in order to achieve maximum throughput, the following condition must hold: $n \geq v T \sigma / A_{\max }$. In other words, below this threshold, it is not possible to achieve maximum throughput.

The maximum achievable throughput as a function of $n$ and $\sigma$ is as follows:

$$
\operatorname{Th}_{A}(n, \sigma)=f \sigma\left(1-\frac{1}{n}\right)^{n-1}
$$

If $n$ is large enough, $n \rightarrow \infty$, Equation (23) can be approximated by the following:

$$
\operatorname{Th}_{A}(n, \sigma) \approx \frac{f \sigma}{e}, n \rightarrow \infty
$$

Therefore, Equation (24) reveals that the length of the coverage area is not relevant for the maximum achievable throughput, and is also consistent with Equation (14).

3.2.4. Generalization of throughput for Arbitrary Frame Lengths

If the frame duration, $t_{f}$, cannot be neglected, equations in Section 3.2 can be generalized to introduce the frame length. Thus, the probability of transmitting within the transmission zone depends on $p_{t x}$ and on the probability of performing a transmission while crossing the transmission zone. 
Moreover, the probability of another nano-node being in the collision zone and not transmitting in a given time slot, $p_{A, n t x}$, can be modeled as follows:

$$
p_{A, n t x}=p_{c x}\left(1-\frac{1}{\sigma}\right)=\frac{A+v t_{f}}{v T}\left(1-\frac{1}{\sigma}\right)
$$

The successful transmission probability to a nano-router, for any nano-node, $p_{A, t x}$, can then be formulated as follows:

$$
p_{A, t x}=p_{t x} \frac{1}{\sigma} \sum_{k=0}^{n-1}\left(\begin{array}{c}
n-1 \\
k
\end{array}\right)\left(1-p_{c x}\right)^{n-1-k} p_{A, n t x}^{k}=\frac{p_{t x}}{\sigma}\left(1-\frac{p_{c x}}{\sigma}\right)^{n-1}=\frac{A-v t_{f}}{v T \sigma}\left(1-\frac{A+v t_{f}}{v T \sigma}\right)^{n-1}
$$

From Equation (26), the network throughput can be derived as $\operatorname{Th}\left(n, A, \sigma, t_{f}\right)=n f \sigma p_{A, t x}$ corresponding to $n$ nano-nodes, and the the total event transmission rate, $f \sigma$ :

$$
\operatorname{Th}\left(n, A, \sigma, t_{f}\right)=\frac{n f\left(A-v t_{f}\right)}{v T}\left(1-\frac{A+v t_{f}}{v T \sigma}\right)^{n-1}
$$

Equation (27) represents the frames per time unit that can reach the nano-router. However maximum throughput does not only depend on the number of frames, but also on the duration of these frames. Thus, throughput is maximized when the transmission channel utilization reaches its maximum.

The number of nano-nodes, $n_{t h}$, maximizing Equation (8), can be easily obtained from $\mathrm{dTh}(n, A, \sigma) / \mathrm{d} n=0$. Thus, $n_{t h, \sigma}$ is as follows:

$$
n_{t h, \sigma}=-\log \left(1-\frac{A+v t_{f}}{v T \sigma}\right)^{-1} \approx \frac{v T \sigma}{A+v t_{f}}, A \ll v T \sigma
$$

As can be observed from Equation (28), the number of nano-nodes required to achieve maximum throughput grows with the number of slots.

3.3. Collision Rate

Beyond the analysis of the network throughput in flow-guided nano-networks, it is also worth studying frame collision behavior.

This analysis is quite straightforward, since a collision occurs when two or more nano-nodes transmit at the same time slot within the coverage area. This probability, $p_{c o l}$, can be modeled as follows:

$$
p_{\text {col }}=\sum_{k=2}^{n}\left(\begin{array}{l}
n \\
k
\end{array}\right) p_{A}^{k}\left(1-p_{A}\right)^{n-k}\left(\sum_{m=2}^{k}\left(\begin{array}{l}
k \\
m
\end{array}\right) \frac{1}{\sigma^{m}}\left(1-\frac{1}{\sigma}\right)^{k-m}\right)
$$

Equation (29) determines the probability of two or more nano-nodes being within the coverage area transmitting simultaneously in the same time slot. Moreover, Equation (29) can be simplified as follows:

$$
p_{c o l}=1-\left(1-\frac{A}{v T \sigma}\right)^{n-1}\left(1+\frac{(n-1) A}{v T \sigma}\right)
$$

Let us define $\operatorname{Col}(n, A, \sigma)=f \sigma p_{\text {col }}$ as the number of collisions per unit of time. That is, a collision might occur with a frequency $f \sigma$ :

$$
\operatorname{Col}(n, A, \sigma)=f \sigma\left(1-\left(1-\frac{A}{v T \sigma}\right)^{n-1}\left(1+\frac{(n-1) A}{v T \sigma}\right)\right)
$$


Demonstrating that $\lim _{n \rightarrow \infty} p_{c o l}=1$ is straightforward; i.e., if there are too many nano-nodes in the flow network, all transmissions within the coverage area will collide.

3.4. Channel Utilization and Frame Length

Regarding channel utilization, $\mathrm{U}\left(n, A, \sigma, t_{f}\right)$, it can be defined as the number of frames per time unit times the frame length (throughput is taken from Equation (27)):

$$
\mathrm{U}\left(n, A, \sigma, t_{f}\right)=\operatorname{Th}\left(n, A, \sigma, t_{f}\right) \times t_{f}=\frac{n f t_{f}\left(A-v t_{f}\right)}{v T}\left(1-\frac{A+v t_{f}}{v T \sigma}\right)^{n-1}
$$

From Equation (32), it is easy to derive the value of $t_{f}, t_{f U}$, which maximizes $\mathrm{U}\left(n, A, \sigma, t_{f}\right)$ An upper bound can be obtained considering the following (as shown in Figure 2):

$$
\mathrm{U}\left(n, A, \sigma, t_{f}\right) \leq \frac{n f t_{f}\left(A-v t_{f}\right)}{v T} \Rightarrow t_{f} u \leq \frac{A}{2 v}
$$

Equations (28) and (33) allow us to define a lower bound of the maximum achievable throughput expressed as channel utilization, as follows:

$$
\max \mathrm{U}\left(n, A, \sigma, t_{f}\right) \geq \frac{A \sigma}{6 v e}
$$

Figure 2 represents an example of channel utilization as a function of the frame length $\left(t_{f}\right)$ and the number of nano-nodes, with fixed values of $A, f, v, T$, and $\sigma$. It can be seen that there is a number of nano-nodes and a frame length that maximize channel utilization. Moreover, it can be observed that the lower bound of the maximum of the channel utilization obtained in Equation (33) is close to its real maximum.

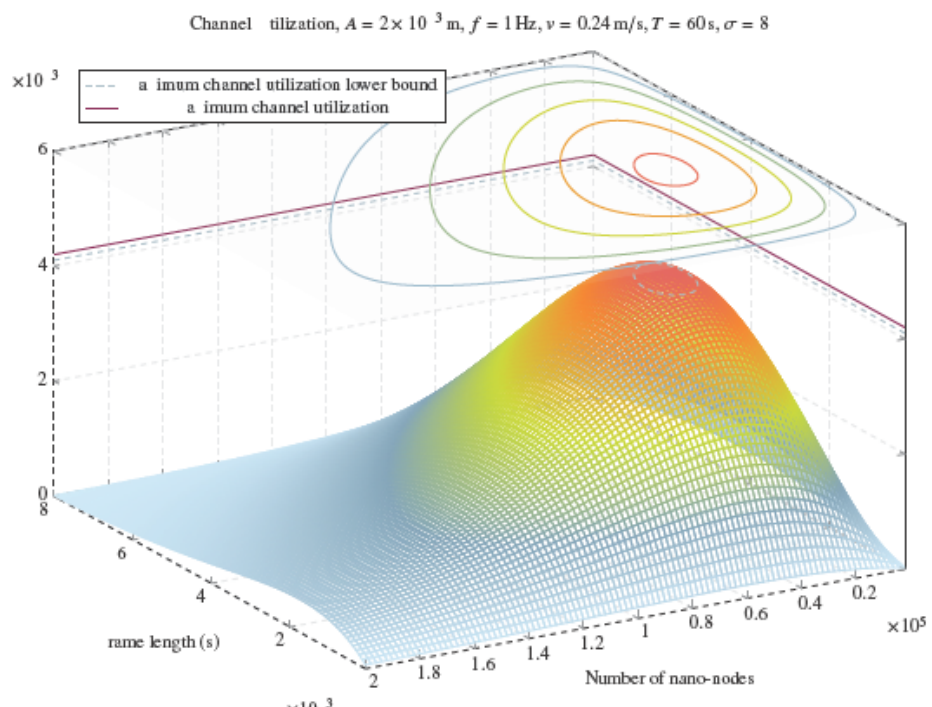

$\times 10^{3}$

Figure 2. Channel utilization as a function of $n$ and $t_{f}$. 


\section{Results}

The analytical model in Section 3 was validated by means of simulations. Network parameters for these simulations are based on those of [7], where the authors modeled a flow-guided nano-network inside the human body. Table 2 specifies these values, including the number of slots used in the simulation.

Table 2. Values of the model parameters used in the simulations.

\begin{tabular}{ll}
\hline Variable & Range of Values \\
\hline$v$ & $0.24 \mathrm{~m} / \mathrm{s}$ \\
$T$ & $60 \mathrm{~s}$ \\
$f$ & $1 \mathrm{~Hz}$ \\
$A$ & $0.5-2 \times 10^{-3} \mathrm{~m}$ \\
$\sigma$ & $1,2,4$ and 8 \\
$t_{f}$ & $0-512 \mu \mathrm{s}$ \\
\hline
\end{tabular}

The aim of these simulations is not to build a very detailed nano-network simulator at the link or physical layers, which is a future goal, but to develop a rigorous transmission model abstracting the following main features:

- Nano-nodes are randomly deployed in a circulatory system of length $v T$.

- There is only one nano-router in the network, although results can proportionally be generalized to more nano-routers, as they are independent.

- The nano-router coverage area is of length $A$.

- Only a single transmission within the coverage of the nano-router is considered to be a successful frame transmission. No frames are received by the nano-router otherwise.

- Two or more simultaneous transmissions within the coverage area always collide.

- Nano-nodes can only send a frame between two battery charges.

- Nano-nodes randomly choose a transmission slot at every transmission cycle.

- Nano-nodes move at an average speed $v$ in the flow.

This model has been programmed in Matlab, and the simulation scenario variables are the number of nano-nodes, the number of slots, the length of the coverage area, the battery charging frequency, the nano-node speed in the flow, the time to complete a round in the flow, and the simulation seed. Every point in the simulations is repeated 10 times with a different seed.

\section{1. throughput Validation}

Figure 3 illustrates a comparison of network throughput using different numbers of slots for every scenario. These simulations assume that $t_{f} \approx 0$. As can be observed, simulations match with the throughput predicted by the analytical model. These plots show that a greater number of slots allows us to reach a larger throughput, but a higher number of nano-nodes is required. In fact, a larger number of nano-nodes without increasing the number of slots would only worsen the achieved throughput, or a greater number of slots without raising the number of nano-nodes would hardly have any effect for any node within the stability zone (i.e., when the collision probability can be still neglected or the number of nano-nodes is not too high).

In the lower part of Figure 3, it can easily be seen that increasing the number of slots allows for a proportional growth of the maximum achievable throughput at the cost of also increasing the number of nano-nodes. This was previously predicted by Equations (10) (the number of nano-nodes that maximize throughput) and (14) (the maximum achievable throughput). 

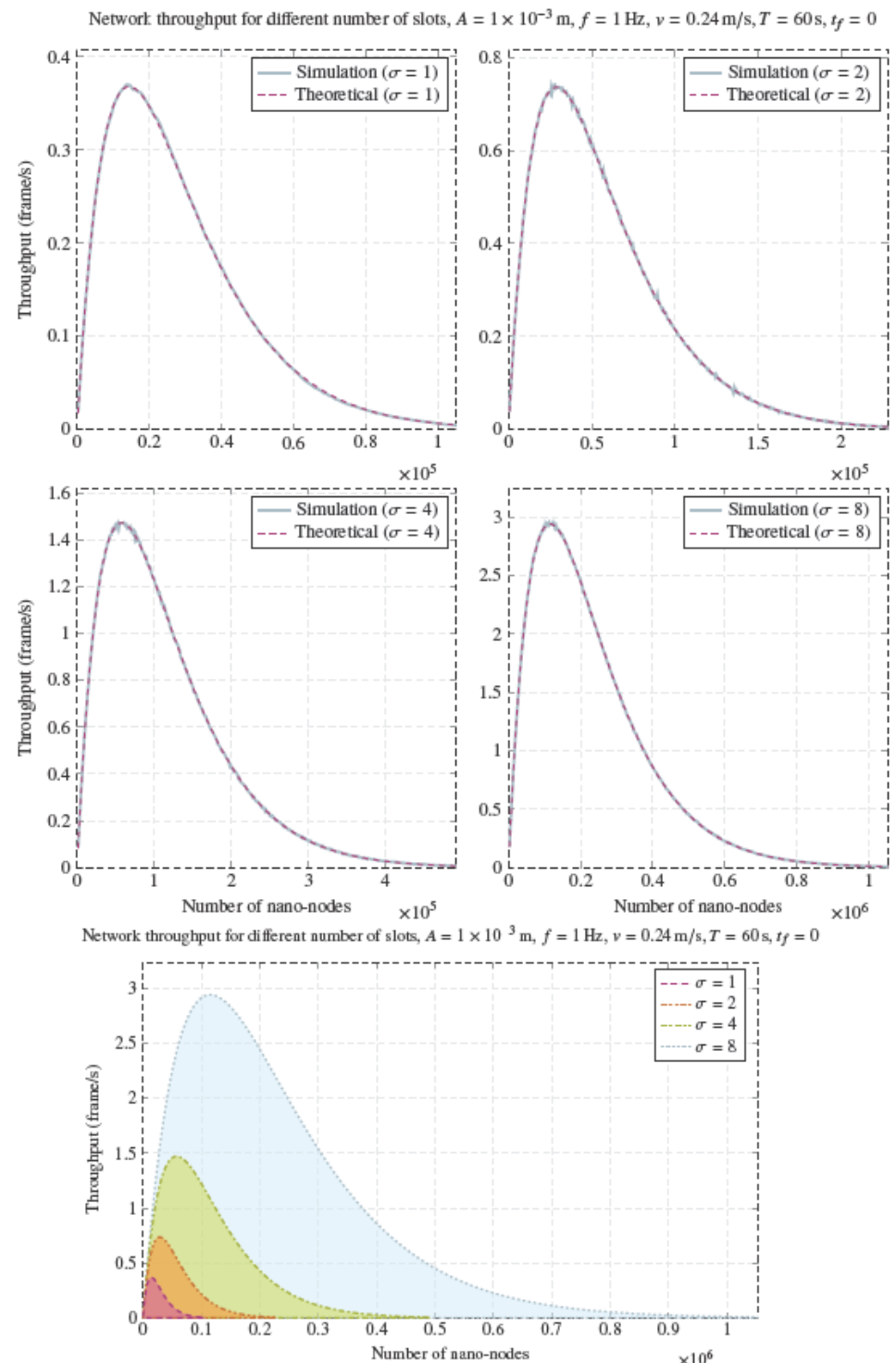

Figure 3. Impact of number of slots on network throughput for $A=1 \times 10^{-3} \mathrm{~m}, f=1 \mathrm{~Hz}, v=0.24 \mathrm{~m} / \mathrm{s}$, $T=60 \mathrm{~s}$, and $t_{f}=0$.

As can be seen, enlarging the number of slots and the number of nano-nodes is the only way to increase the maximum achievable throughput. It might be possible that the number of nano-nodes is 
fixed; consequently, independently of the number of slots, the maximum throughput will be upper bounded by $n A f / v T$, in accordance with Equation (8). This happens when $\sigma \rightarrow \infty$; i.e., for an arbitrary number of nano-nodes, the maximum throughput is achieved when $\sigma \rightarrow \infty$. Consequently, in any flow-guided nano-network setup, the largest $\sigma$ possible should be used. The calculation of this value is beyond the scope of this paper, but it depends on the maximum battery charging frequency and the frame length.

Regarding the length of the coverage area, $A$, Figure 4 plots the simulation results for the achieved throughput using different lengths of the coverage area, maintaining the remaining parameters constant. These simulations also validate the outcomes predicted by theory, and, as can be observed, the length of the coverage area has no significant influence on the maximum achievable throughput. However, lower lengths of the coverage area require more nano-nodes to achieve maximum throughput, as was unveiled by Equations (10) and (11). This can be more easily observed in the lower part of Figure 4.

Network throughput for different lengths of the coverage area, $f=1 \mathrm{~Hz}, v=0.24 \mathrm{~m} / \mathrm{s}, T=60 \mathrm{~s}, \sigma=2, t_{f}=0$
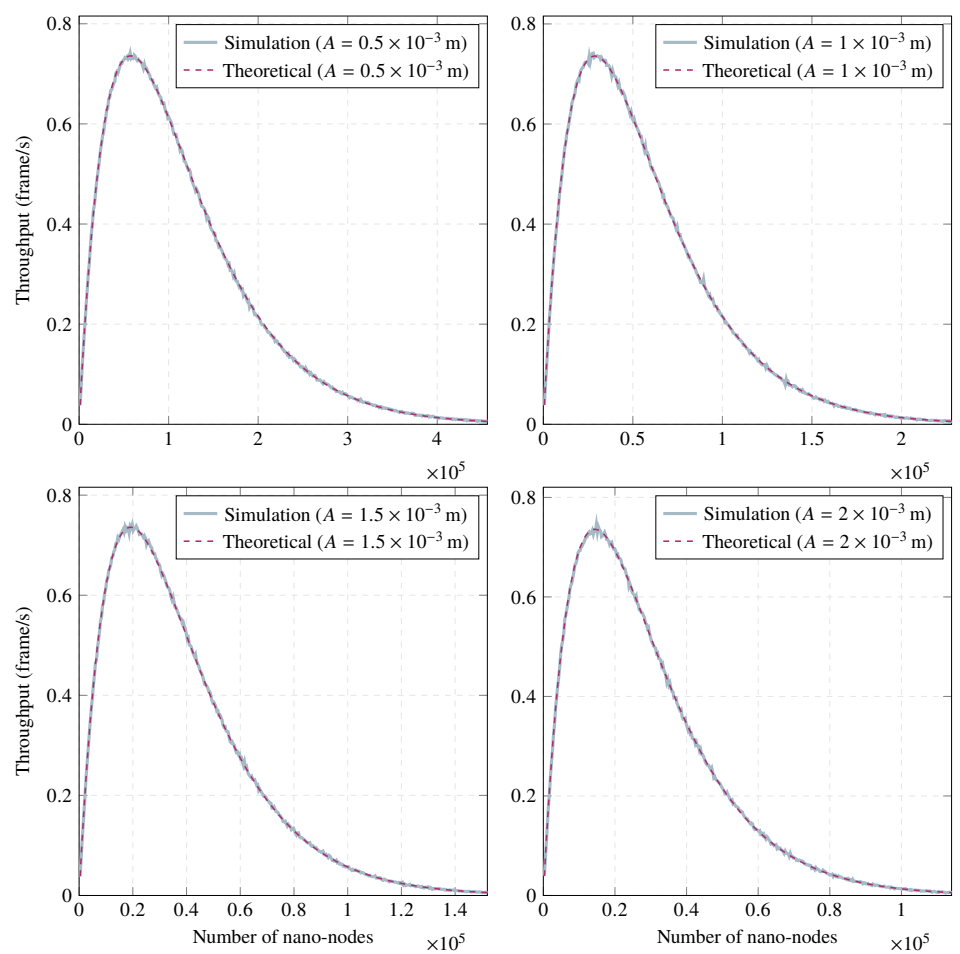

Figure 4. Cont 


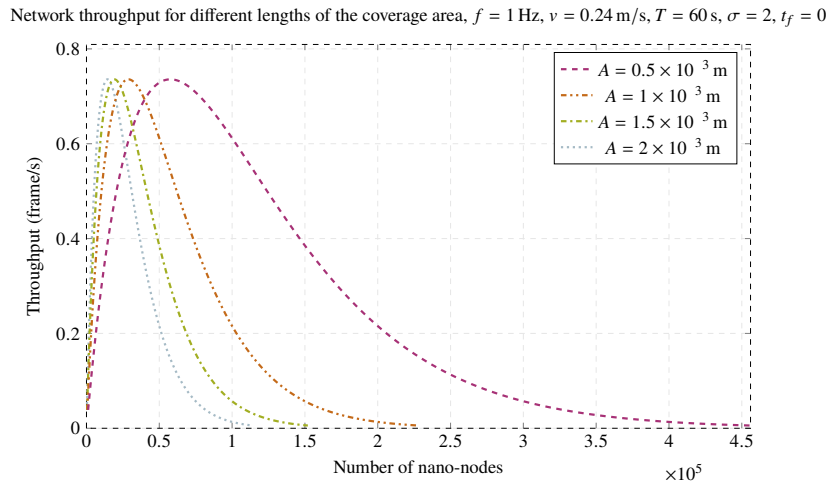

Figure 4. Influence of the length of the coverage area on network throughput for $f=1 \mathrm{~Hz}, v=0.24 \mathrm{~m} / \mathrm{s}$,

$$
T=60 \mathrm{~s}, \sigma=2 \text {, and } t_{f}=0 \text {. }
$$

The intuition behind these findings is that, by choosing larger lengths of the coverage area, a better solution for this type of network exists since fewer numbers of nano-nodes are required to achieve maximum throughput. However, albeit outside the scope of this paper, other parameters must be taken into consideration, such as the network lifetime and the average nano-node lifespan set in the network design. In order to configure a flow-guided nano-network with a given lifetime, it may be necessary to use a larger number of nano-nodes, which as theory and simulations indicate, could lead to poor throughputs. To avoid this, a possible solution could be the use of lower transmission powers in the nano-nodes to reduce the length of their coverage range $(A)$ and, as nano-nodes start dying, increase it to maximize throughput during the network lifetime.

Figure 5 represents the relationship between the length of the coverage area, the number of slots, the number of nano-nodes, and the nano-network throughput. Increasing or decreasing $A$ causes more or fewer nano-nodes to be required to achieve maximum throughput. Moreover, increasing the number of slots allows us to achieve higher network throughput at the expense of proportionally increasing the number of nano-nodes. As mentioned above, Figure 5 shows that the length of the coverage area is not significant to achieve maximum throughput. To this end, a proper setup of the required amount of nano-nodes and the number of slots is sufficient.

Finally, frame length impact on throughput can be observed in Figure 6. It illustrates the achieved throughput for the same scenario for different frame lengths. As can be expected, a longer frame length reduces network throughput as a consequence of increasing the collision area and decreasing the transmission area. However, frame length should be neglected in most cases; if $A \gtrsim v t_{f}$, network throughput reduction can be significant. To this end, larger values of $A$ can provide better network throughput values, as can be seen in the lower part of Figures 6 and 7. 

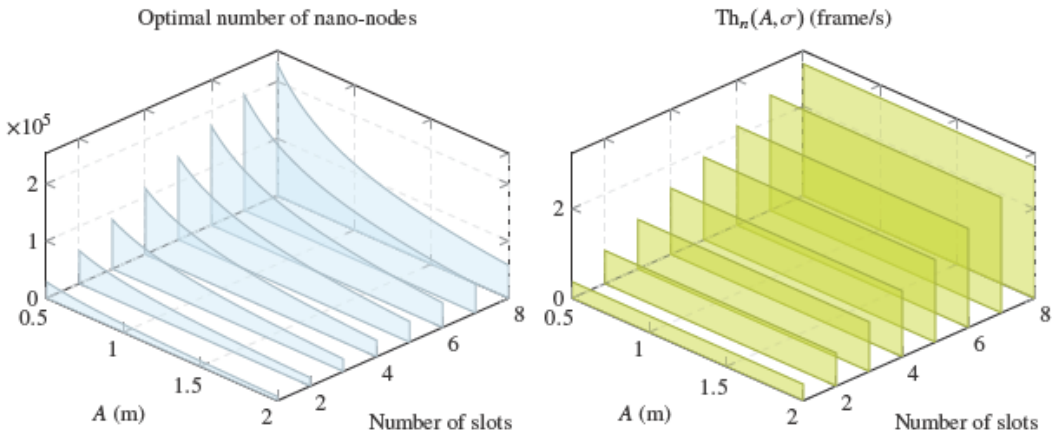

$\times 10^{-3}$

$\times 10^{-3}$

Figure 5. Relation between the optimal number of nano-nodes and the maximum achievable network throughput for $f=1 \mathrm{~Hz}, v=0.24 \mathrm{~m} / \mathrm{s}, T=60 \mathrm{~s}$, and $t_{f}=0$.

Network throughput for different frame lengths, $A=0.5 \times 10^{-3} \mathrm{~m}, f=1 \mathrm{~Hz}, v=0.24 \mathrm{~m} / \mathrm{s}, T=60 \mathrm{~s}, \sigma=1$
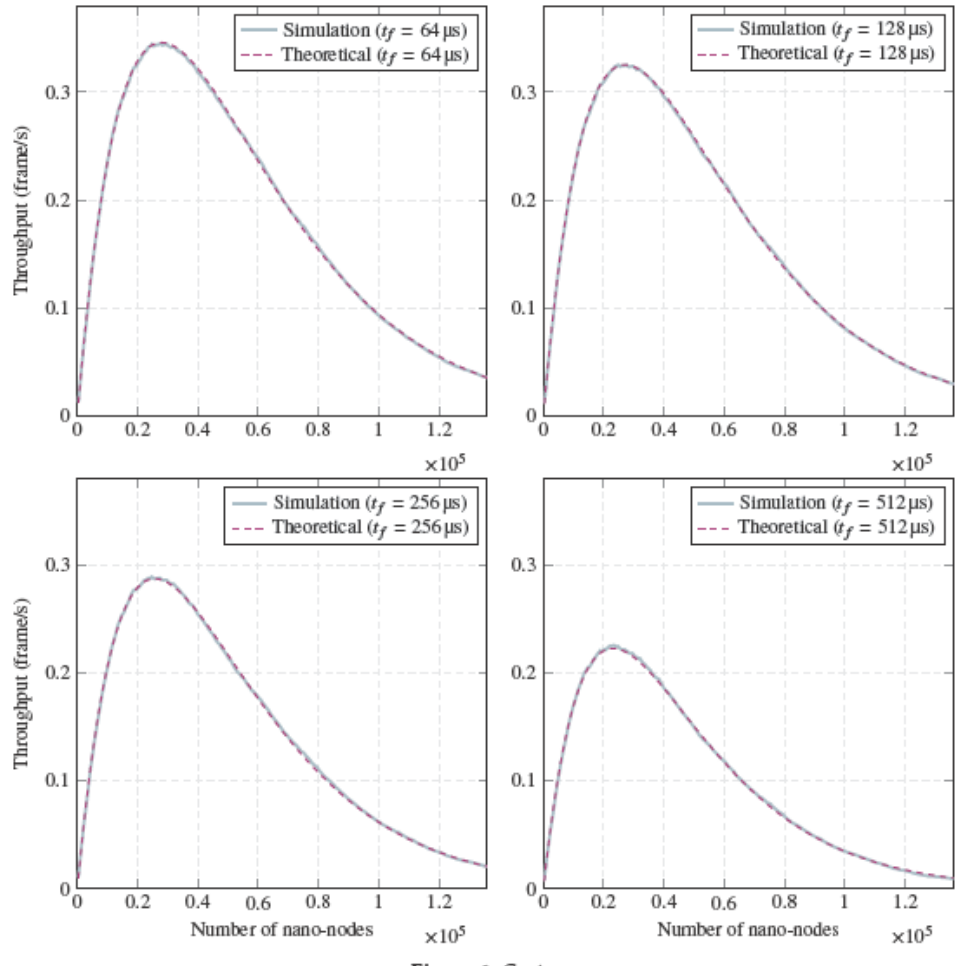

Figure 6. Cont. 


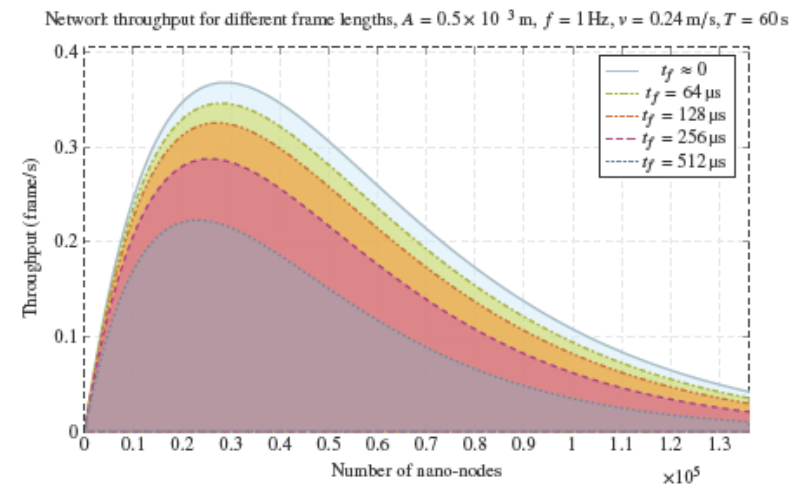

Figure 6. Network throughput for different frame lengths for $A=0.5 \times 10^{-3} \mathrm{~m}, f=1 \mathrm{~Hz}, v=0.24 \mathrm{~m} / \mathrm{s}$, $T=60 \mathrm{~s}$, and $\sigma=1$.

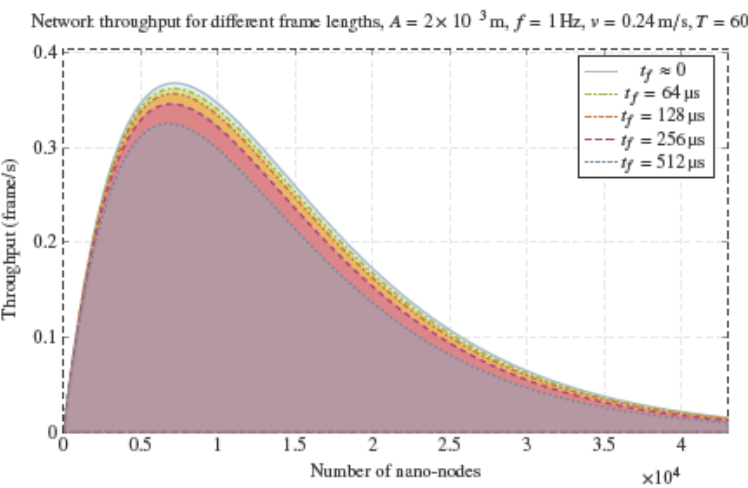

Figure 7. Network throughput for different frame lengths for $A=2 \times 10^{-3} \mathrm{~m}, f=1 \mathrm{~Hz}, v=0.24 \mathrm{~m} / \mathrm{s}$, $T=60 \mathrm{~s}$, and $\sigma=1$.

\subsection{Collision Rate Validation}

The frame collision rate has also been validated by means of computer simulations. Figure 8 depicts the behavior of the collision rate as the number of nano-nodes grows for different numbers of slots, keeping the rest of the parameters constant. Simulations also validate results provided by the analytical model. As can be seen, in all cases the collision rate converges to $f \sigma$ as the number of nano-nodes escalates. In other words, a frame collision occurs with high probability for any transmission attempt (in any time slot) as the number of nano-nodes increases.

Regarding the influence of varying the length of the coverage area on the collision rate, Figure 9 shows several simulation results for different lengths of the coverage area. Again, simulation results validate those obtained by the analytical model. As shown in Figure 9, lower lengths of the coverage area reduce the collision rate for a lower number of nano-nodes. However, all studied scenarios converge to the maximum collision rate as the number of nano-nodes increases. Therefore, if frame collision reduction is the main design concern, it might be interesting to reduce $A$, although less throughput will be achieved. 

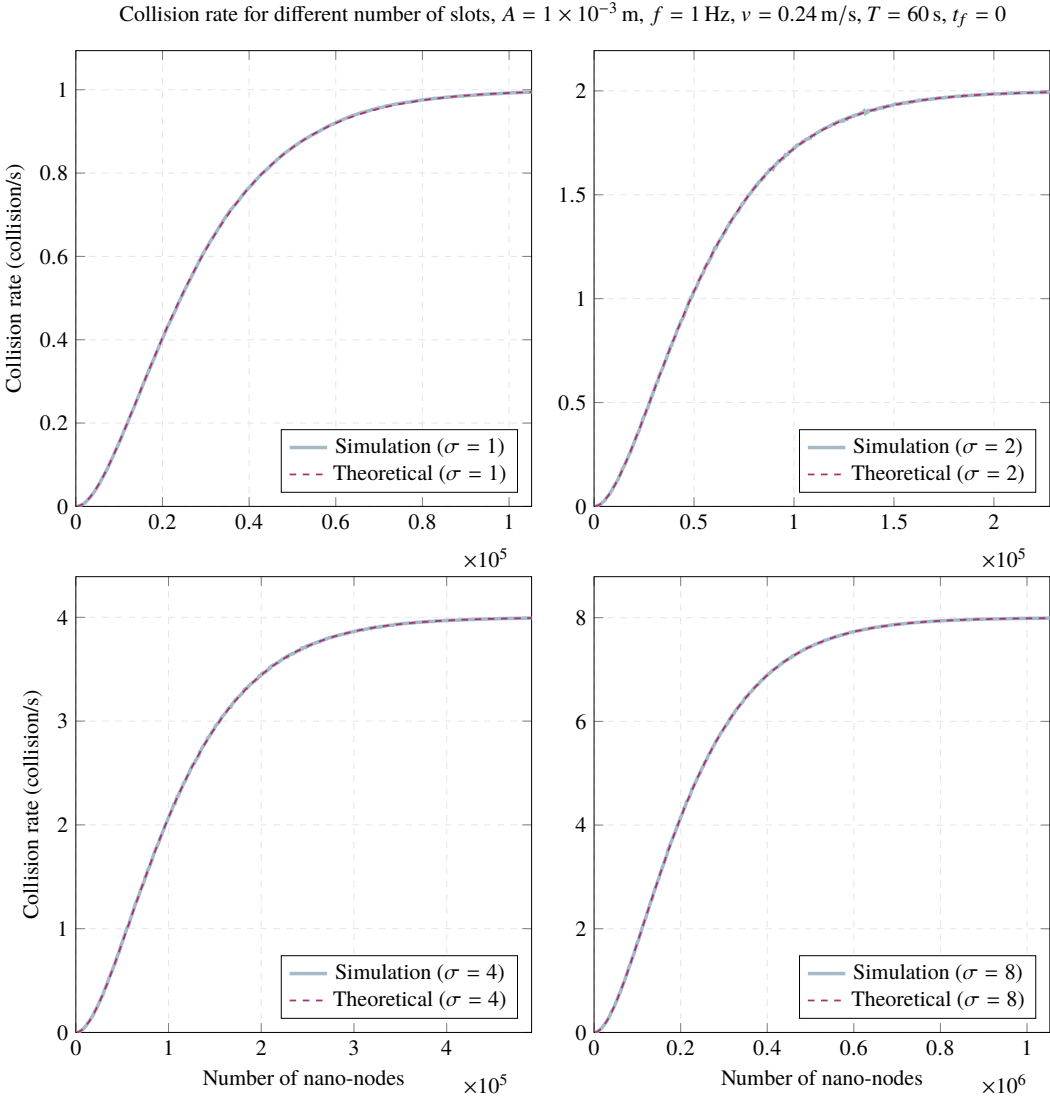

Figure 8. Impact of slots on the network collision rate for $A=1 \times 10^{-3} \mathrm{~m}, f=1 \mathrm{~Hz}, v=0.24 \mathrm{~m} / \mathrm{s}$, $T=60 \mathrm{~s}$, and $t_{f}=0$. 
Collision rate for different lengths of the coverage area, $f=1 \mathrm{~Hz}, v=0.24 \mathrm{~m} / \mathrm{s}, T=60 \mathrm{~s}, \sigma=2, t_{f}=0$
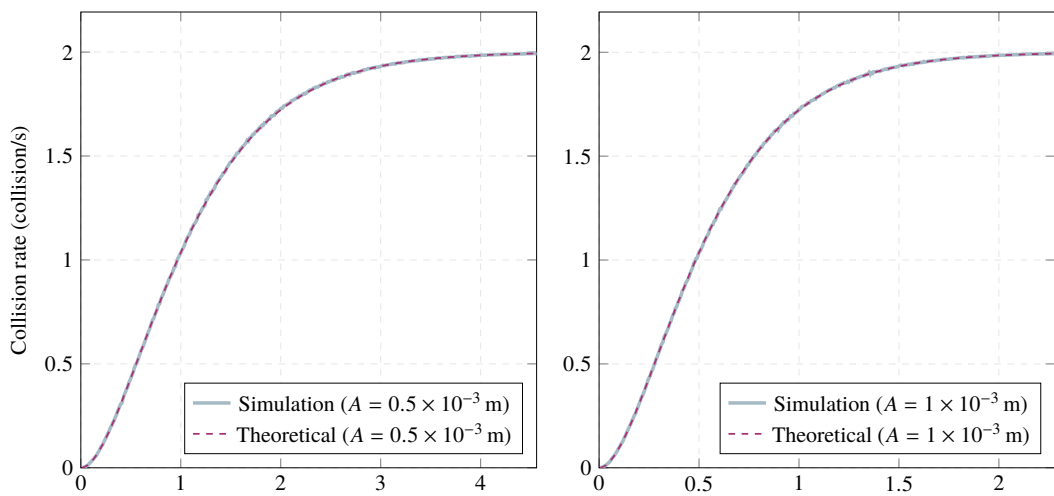

$\times 10^{5}$
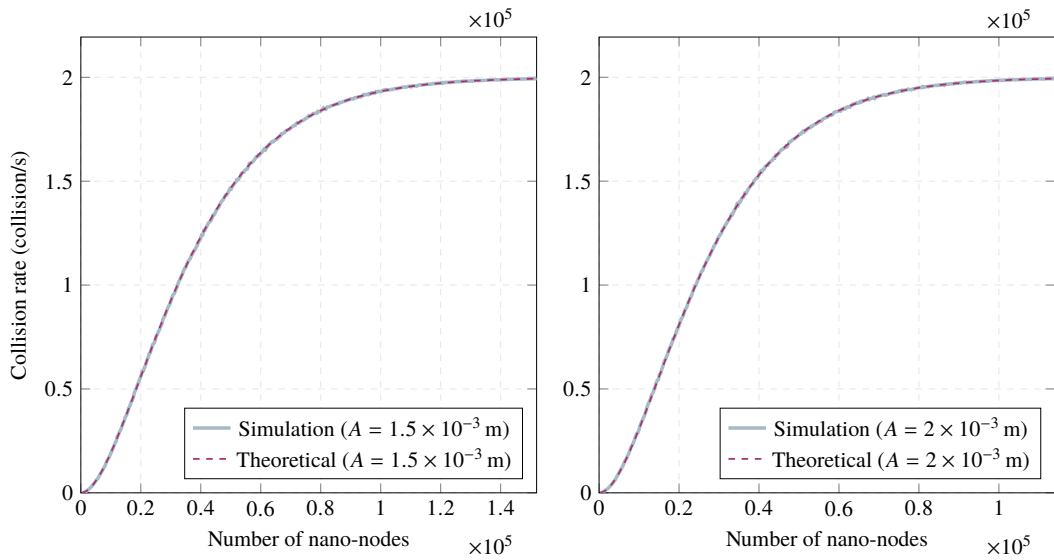

Figure 9. Impact of the length of the coverage area on the network collision rate for $A=1 \times 10^{-3} \mathrm{~m}$ $f=1 \mathrm{~Hz}, v=0.24 \mathrm{~m} / \mathrm{s}, T=60 \mathrm{~s}, \sigma=2$, and $t_{f}=0$.

\section{Conclusions and Future Directions}

This paper establishes a theoretical framework to analyze flow-guided nanocommunication networks. It is further validated by means of computer simulations. The mathematical model presented enables the evaluation of the main metrics of flow-guided nano-networks, such as the network throughput or the frame collision rate, as a function of the number of nano-nodes, the coverage range of nano-routers, the battery recharging frequency, the average nano-node velocity, and the number of transmission slots. Results revealed the existence of a limit for the maximum achievable throughput, which depends mainly on the charging frequency of the batteries and the number of available time transmission slots during the charging cycles. In addition, to reach this limit, an optimal number of nano-nodes in the network are required. Below this limit, the addition of more nano-nodes in the network translates into more successful transmissions and, consequently, a throughput increase. Above this limit, the occurrence of collisions becomes more likely, leading to a throughput reduction. This unstable behavior makes throughput tend toward 0 , due to the saturation of the channel. 
It is also shown that the length of the coverage area has no significant impact on the maximum achievable throughput. However, the optimal number of nano-nodes deployed throughout the network depends on this length. The larger the length of the coverage area is, the fewer nano-nodes are needed to reach the maximum achievable throughput.

Frame length can be neglected if the length of the coverage area is large enough. However, a combination of long frames and a reduced coverage area may have a significant impact on reducing the maximum achievable throughput. Moreover, frame length is important in order to maximize channel utilization. Thus, a proper frame length and the right number of nano-nodes can maximize channel utilization.

Regarding the number of slots, only increasing their number does not imply a substantial improvement in network performance. To have a noticeable effect on network throughput, it is necessary to increase the number of nano-nodes in the same proportion

Simulations presented in this paper should be observed as a first approach to validate our analytical model. In this sense, we are currently developing a simulation framework based on BitSimulator, a wireless nanonetwork simulator $[21,22]$ that is designed to gracefully scale and support millions of nano-nodes. BitSimulator enables the exploration and understanding of the effects of low-level coding and channel access contention. Our aim is to work with simulation models that consider issues that are difficult to model and thus have not been included in the analytical model.

This work represents a step forward in the effort to develop innovative (realistic) applications based on flow-guided nano-networks. It should also be taken as a basis to enable the implementation of feasible protocols for these networks.

\section{Appendix A. The Average Number of Colliding Transmissions}

Section 3.3 offers an analytical equation to calculate the frame collision rate in a flow-guided nanocommunication network, $\operatorname{Col}(n, A, \sigma)$. Let us define $p_{c o l, k}$ as the probability of $k$ nano-node transmissions colliding in the coverage area, $k \geq 2$. From Equation (29), $p_{c o l, k}$ can be expressed as follows:

$$
p_{c o l, k}=\left(\begin{array}{l}
n \\
k
\end{array}\right) p_{A}^{k}\left(1-p_{A}\right)^{n-k}\left(\sum_{m=2}^{k}\left(\begin{array}{c}
k \\
m
\end{array}\right) \frac{1}{\sigma^{m}}\left(1-\frac{1}{\sigma}\right)^{k-m}\right)
$$

Equation (A1) can be further simplified to the following:

$$
p_{c o l, k}=\left(\begin{array}{l}
n \\
k
\end{array}\right)\left(\frac{A}{v T}\right)^{k}\left(1-\frac{A}{v T}\right)^{n-k}\left(1-\frac{k}{\sigma}\left(1-\frac{1}{\sigma}\right)^{k-1}-\left(1-\frac{1}{\sigma}\right)^{k}\right)
$$

Figure A1 depicts an example of the probability of simultaneous transmissions (i.e., collisions) as a function of $n$. As can be expected, frame collisions grow with $n$.

From Equation (A1), it is possible to obtain the average number of collisions as a function of $n$ :

$$
\mathrm{E}\left[p_{c o l, k}\right]=\sum_{k=2}^{n} k\left(\begin{array}{l}
n \\
k
\end{array}\right) p_{A}^{k}\left(1-p_{A}\right)^{n-k}\left(\sum_{m=2}^{k}\left(\begin{array}{l}
k \\
m
\end{array}\right) \frac{1}{\sigma^{m}}\left(1-\frac{1}{\sigma}\right)^{k-m}\right)
$$

Equation (A3) can be simplified as follows:

$$
\mathrm{E}\left[p_{c o l, k}\right]=\frac{n A}{v T}\left(1-\left(1-\frac{A}{v T \sigma}\right)^{n-1}\left(1-\frac{1}{\sigma}\right)-\frac{1}{\sigma}\left(1-\frac{A}{v T \sigma}\right)^{n-2}\right)
$$

If $n$ is large enough, $n \rightarrow \infty, \mathrm{E}\left[p_{c o l, k}\right]$ can be approximated by the following:

$$
\mathrm{E}\left[p_{c o l, k}\right] \approx \frac{n A}{v T}, n \rightarrow \infty
$$


Figure A2 illustrates the behavior of the average number of collisions in a transmission slot. If $n$ is large enough, $\mathrm{E}\left[p_{c o l k}\right]$ can be approximated by a linear function of slope $n A / v T$.

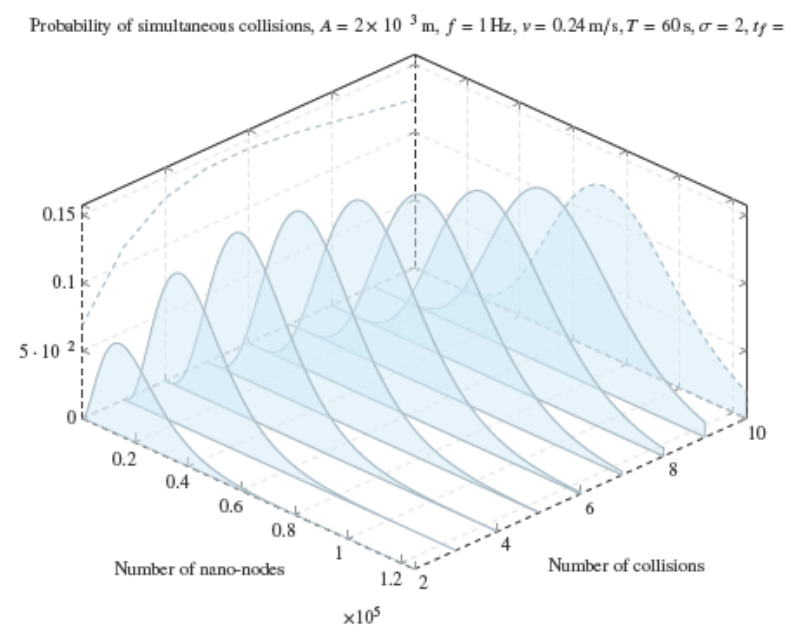

Figure A1. Probability of frame collisions for $A=2 \times 10^{-3} \mathrm{~m}, f=1 \mathrm{~Hz}, \sigma=2$, and $t_{f}=0$.

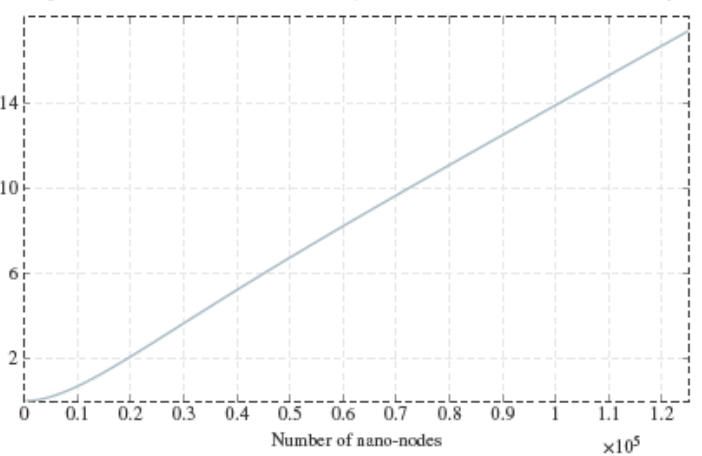

Figure A2. Average number of collisions for $A=2 \times 10^{-3} \mathrm{~m}, f=1 \mathrm{~Hz}, \sigma=2$, and $t_{f}=0$.

Author Contributions: Conceptualization: R.A.-C. and S.C.-C.; methodology: R.A.-C. and S.C.-C.; software: R.A.-C.; validation: S.C.-C. and A.-J.G.-S.; writing -original draft preparation: R.A.-C. and S.C.-C.; writing-review and editing: A.-J.G.-S. and J.G.-H.; supervision: J.G.-H; project administration: J.G.-H.; funding acquisition: J.G.-H. All authors have read and agreed to the published version of the manuscript.

Funding: This research was funded by the projects AIM, ref. TEC2016-76465-C2-1-R (AEI/FEDER, UE), e-DIVITA, ref.20509/PDC/18 (Proof of Concept, 2018), and ATENTO, ref. 20889/PI/18 (Fundación Séneca, Región de Murcia).

Acknowledgments: Sebastian Canovas-Carrasco thanks the Spanish MECD for an FPU (ref. FPU16/03530) pre-doctoral fellowship. 
Conflicts of Interest: The authors declare that there is no conflict of interest. The funders had no role in the design of the study; in the collection, analyses, or interpretation of data; in the writing of the manuscript; or in the decision to publish the results.

\section{References}

1. Stelzner, M.; Dressler, F.; Fischer, S. Function Centric Nano-Networking: Addressing nano machines in a medical application scenario. Nano Commun. Netw. 2017, 14, 29-39. doi:10.1016/j.nancom.2017.09.001. [CrossRef]

2. Akyildiz, I.F.; Jornet, J.M. Electromagnetic wireless nanosensor networks. Nano Commun. Netw. 2010, 1, 3-19. doi:10.1016/j.nancom.2010.04.001. [CrossRef]

3. Piro, G.; Bia, P.; Boggia, G.; Caratelli, D.; Grieco, L.; Mescia, L. Terahertz electromagnetic field propagation in human tissues: A study on communication capabilities. Nano Commun. Netw. 2016, 10, 51-59. doi:10.1016/j.nancom.2016.07.010. [CrossRef]

4. Chahibi, Y. Molecular communication for drug delivery systems: A survey. Nano Commun. Netw. 2017, 11, 90-102. doi:10.1016/j.nancom.2017.01.003. [CrossRef]

5. Felicetti, L.; Femminella, M.; Reali, G.; Liò, P. Applications of molecular communications to medicine: A survey. Nano Commun. Netw. 2016, 7, 27-45. doi:10.1016/j.nancom.2015.08.004. [CrossRef]

6. Kosaka, P.M.; Pini, V.; Ruz, J.J.; da Silva, R.A.; González, M.U.; Ramos, D.; Calleja, M.; Tamayo, J. Detection of cancer biomarkers in serum using a hybrid mechanical and optoplasmonic nanosensor. Nat. Nanotechnol. 2014, 9, 1047. [CrossRef] [PubMed]

7. Canovas-Carrasco, S.; Sandoval, R M.; Garcia-Sanchez, A.; Garcia-Haro, J. Optimal Transmission Policy Derivation for IoNT Flow-guided Nano-sensor Networks. IEEE Internet Things J. 2019, 6, 2288-2298. doi:10.1109/JIOT.2019.2906015. [CrossRef]

8. Canovas-Carrasco, S.; Garcia-Sanchez, A.J.; Garcia-Haro, J. The IEEE 1906.1 Standard: Some Guidelines for Strengthening Future Normalization in Electromagnetic Nanocommunications. IEEE Commun. Stand. Mag. 2018, 2, 26-32. doi:10.1109/MCOMSTD.2018.1700082. [CrossRef]

9. Abbasi, Q.H.; El Sallabi, H.; Chopra, N.; Yang, K.; Qaraqe, K.A.; Alomainy, A. Terahertz Channel Characterization Inside the Human Skin for Nano-Scale Body-Centric Networks. IEEE Trans. Terahertz Sci. Technol. 2016, 6, 427-434. doi:10.1109/TTHZ.2016.2542213. [CrossRef]

10. Yang, K.; Pellegrini, A.; Munoz, M.O.; Brizzi, A.; Alomainy, A.; Hao, Y. Numerical Analysis and Characterization of $\mathrm{THz}$ Propagation Channel for Body-Centric Nano-Communications. IEEE Trans Terahertz Sci. Technol. 2015, 5, 419-426. doi:10.1109/TTHZ.2015.2419823. [CrossRef]

11. Canovas-Carrasco, S.; Garcia-Sanchez, A.J.; Garcia-Haro, J. A nanoscale communication network scheme and energy model for a human hand scenario. Nano Commun. Netw. 2018, 15, 17-27. doi:10.1016/j.nancom.2018.01.005. [CrossRef]

12. Canovas-Carrasco, S.; Garcia-Sanchez, A.J.; Garcia-Sanchez, F.; Garcia-Haro, J. Conceptual Design of a Nano-Networking Device. Sensors 2016, 16, 2104. doi:10.3390/s16122104. [CrossRef]

13. Jornet, J M.; Akyildiz, I.F. Joint Energy Harvesting and Communication Analysis for Perpetual Wireless Nanosensor Networks in the Terahertz Band. IEEE Trans. Nanotechnol. 2012, 11, 570-580. doi:10.1109/TNANO.2012.2186313. [CrossRef]

14. Canovas-Carrasco, S.; Garcia-Sanchez, A.J.; Garcia-Haro, J. On the Nature of Energy-Feasible Wireless Nanosensor Networks. Sensors 2018, 18, 1356. doi:10.3390/s18051356. [CrossRef]

15. Akyildiz, I.; Jornet, J. The Internet of nano-things. IEEE Wirel. Commun. 2010, 17, 58-63. doi:10.1109/MWC.2010.5675779. [CrossRef]

16. Singh, P.; Kim, B.W.; Jung, S.Y. TH-PPM with non-coherent detection for multiple access in electromagnetic wireless nanocommunications. Nano Commun. Netw. 2018, 17, 1-13. doi:10.1016/j.nancom.2018.05.001 [CrossRef]

17. Afsana, F.; Asif-Ur-Rahman, M.; Ahmed, M.R.; Mahmud, M.; Kaiser, M.S. An Energy Conserving Routing Scheme for Wireless Body Sensor Nanonetwork Communication. IEEE Access 2018, 6, 9186-9200. doi:10.1109/ACCESS.2018.2789437. [CrossRef]

18. Yu, H.; Ng, B.; Seah, W.K.G. TTL-based Efficient Forwarding for Nanonetworks with Multiple Coordinated IoT Gateways. IEEE Internet Things J. 2018, 5, 1807-1815. doi:10.1109/JIOT.2018.2812868. [CrossRef] 
19. Pierobon, M.; Jornet, J M.; Akkari, N.; Almasri, S.; Akyildiz, I.F. A routing framework for energy harvesting wireless nanosensor networks in the Terahertz Band. Wirel. Netw. 2014, 20, 1169-1183. doi:10.1007/s11276-013-0665-y. [CrossRef]

20. Yao, X.; Wang, C.; Wang, W.; Jornet, J.M. On the Achievable throughput of Energy-Harvesting Nanonetworks in the Terahertz Band. IEEE Sens. J. 2018, 18, 902-912. doi:10.1109/JSEN.2017.2776301. [CrossRef]

21. Dhoutaut, D.; Arrabal, T.; Dedu, E. Bit Simulator, an Electromagnetic Nanonetworks Simulator. In Proceedings of the 5th ACM International Conference on Nanoscale Computing and Communication; Association for Computing Machinery: New York, NY, USA, 2018; NANOCOM '18. doi:10.1145/3233188.3233205. [CrossRef]

22. BitSimulator, a C++ Wireless Nanonetwork Simulator for Routing and Transport Levels. Available online: http://eugen.dedu.free.fr/bitsimulator/ (accessed on 22 February 2020).

(C) 2020 by the authors. Licensee MDPI, Basel, Switzerland. This article is an open access article distributed under the terms and conditions of the Creative Commons Attribution (CC BY) license (http://creativecommons.org/licenses/by/4.0/). 



\section{Part III}

\section{Summary of Articles}





\section{Chapter 1}

\section{Conceptual Design of a Nano-Networking Device}

\subsection{Brief summary}

By the age of 2016, when this thesis started, all works published in the field were not based on specific nano-devices with quantitative specifications, so this lack of a common framework made that each author considered the limitations of a nano-network differently (in terms of energy harvesting and storage, memory available, processing capacity or communication range), resulting in very disparate works. Therefore, the objective of this first paper was to know and better understand the electronics associated with the main components required for a nano-device, its layout, and key specifications, so that we provided the scientific community with a grounded starting point for future works in the field. To this effect, we developed a thorough state-of-the-art review for each nano-device component, discussing different available technologies (commercial or pre-commercial), and selecting the most suitable for each of them. To complete the conceptual layout of the nano-device, we considered the nanodevice size equal to a red blood cell (i.e., an ultra-small size) to dimension and allocate the components over the nano-device footprint.

\subsection{Methodology and tools}

The methodology followed in this paper was divided into the following steps:

1. Documentation and study of the most advanced technologies available today (e.g. FinFet transistors, A-RAM memories, or graphene-based antennas) for the design of the main components of a nanodevice (processor, memories, power generator, antenna, communication transceiver, and sensor.).

2. Documentation and study of the experimental technologies that, a priori, will be commercially implemented in the future (e.g. atomic tran- 
sistors, carbon nanotubes, racetrack memory, giant magnetoresistance based memories, etc.) for the design of each of the nanodevice components.

3. Based on all the information collected about each technology and considering the features that a nano-device should have, we selected the most suitable technology for each component of the nano-device.

4. Design of the nano-device layout dimensioning each component in order to balance their performance so that (i) the bottlenecks in the design are mitigated and (ii) the nano-devices have sufficient capacity to operate in a WNSN.

5. Once all the components were conceptually designed, a deep study of their expected power consumption was developed.

\subsection{Results and Contributions}

The features of each component of the designed nano-device are shown in Table 1.1 .

Results show that the nano-device designed based on current and feasible technologies has sufficient capability to run ad-hoc communication protocols and perform simple tasks, so we went one significant step further in the feasibility and potential of nano-communications. Besides, we can divide the contributions of this work into two main parts:

1. We provided an insightful state-of-the-art review to select the appropriate technological solutions for all the components comprising a nano-device.

2. We conceptualized the nanodevice design, quantifying its dimensions, and the core features components integrating a functional nano-device. Special attention was paid to the communication tasks that a generic nano-device has to perform in a WNSN.

\subsection{Conclusions}

A conceptual design of a nano-device with communication capabilities in the $\mathrm{THz}$ band was conducted in this work. To this end, we analyzed the requirements of the main components in a potential EM nano-device and then reviewed and discussed the advantages and disadvantages of different current and future technologies. Then, we selected the most suitable one, according to its appropriateness to the nanodevice capabilities and size. Based on the obtained results, we can conclude that a red-blood-cell-sized device has the potential to run simple nano-communication protocols and perform simple tasks in scenarios where larger artificial devices would be excessively invasive. This paper entailed an excellent starting point for future works in this thesis. 
CHAPTER 1. CONCEPTUAL DESIGN OF A NANO-NETWORKING

Table 1.1: Conceptual nano-device features for each component.

\begin{tabular}{|c|c|c|c|c|}
\hline Component & Main feature & $\begin{array}{l}\text { Footprint } \\
\qquad(\mu m)\end{array}$ & $\begin{array}{l}\text { Technology } \\
\text { employed }\end{array}$ & $\begin{array}{c}\text { Power } \\
\text { consumption }\end{array}$ \\
\hline Processor & $\begin{array}{c}500 \mathrm{kHz} \text { with } \\
5000 \mathrm{MOSFET}\end{array}$ & $5 \times 5$ & SiGe transistors & \multirow{3}{*}{$140 \mathrm{nW}$} \\
\hline $\begin{array}{l}\text { Read-Only } \\
\text { Memory }\end{array}$ & 386 bytes & $2 \times 2$ & NOR memory & \\
\hline $\begin{array}{l}\text { Random-Access } \\
\text { Memory }\end{array}$ & 1275 bytes & $3 \times 4$ & A-RAM & \\
\hline Flash Memory & 256 bytes & $1 \times 2$ & $\begin{array}{l}\text { NAND SLC } \\
\text { memory }\end{array}$ & $30 \mathrm{pJ} / \mathrm{bit}$ \\
\hline $\begin{array}{l}\text { Communication } \\
\text { module }\end{array}$ & $\begin{array}{c}\text { Operation } \\
\text { frequency: } 1 \\
\mathrm{THz}\end{array}$ & $5 \times 3$ & $\begin{array}{l}\mathrm{SiO} 2 \text { and } \\
\text { graphene }\end{array}$ & $1 \mathrm{uW}$ \\
\hline Sensor & - & $3 \times 2$ & $\begin{array}{l}\text { Depends on the } \\
\text { application }\end{array}$ & $50 \mathrm{nW}$ \\
\hline Generator & $\begin{array}{c}\text { Energy } \\
\text { harvesting: } 2.46 \\
\mathrm{nW}\end{array}$ & $8 \times 8$ & $\mathrm{ZnO}$ nanowires & - \\
\hline Battery & $\begin{array}{c}\text { Energy stored: } \\
70.4 \mathrm{nF}\end{array}$ & $8 \times 8$ & $\begin{array}{c}\text { Electric } \\
\text { double-layer } \\
\text { (EDLC) } \\
\text { capacitor }\end{array}$ & - \\
\hline
\end{tabular}





\section{Chapter 2}

\section{The IEEE 1906.1 Standard: Nanocommunications as a new source of data}

\subsection{Brief summary}

The lack of a common framework in EM nano-communications was leading to isolated works and unrelated knowledge islands, hindering a better and quicker advance in the field. Thus, works published in the scientific literature were conceived and evaluated under very specific (and sometimes unrealistic) conditions, being very uneven from one paper to another. In this context, the IEEE 1906.1 standard 8 was released in December 2015 to join efforts and promote more cooperation between research groups. It provides a normalized protocol stack that should be implemented by each nano-device working in a WNSN and a set of standardized metrics to evaluate the performance of a WNSN. However, based on the know-how about EM nano-communications acquired the first work of the thesis, we detected several lacks and weaknesses in the IEEE 1906.1 standard. Most of them were derived from the desire to cover the two types of nano-communications (i.e. molecular and EM) under the umbrella of the same standard, even though there are significant differences between them. This made the standard too generic for EM nano-communications, steering away from its original goal. Thus, in this work, we discussed the pros and cons of the standard from the perspective of EM communications and specify how to tackle each detected weakness in order to make the standard useful and acceptable by the scientific community when designing EM nano-communications protocols.

\subsection{Methodology and tools}

The methodology of this work was divided into three main parts: 
1. In-depth analysis of the IEEE 1906.1 standard and envisage how it can be applied to (i) the design of generic nano-communication protocols or (ii) WNSN for specific applications. We even contacted one of the persons in charge of developing the standard, Stephen F. Bush, to clarify how to proceed when applying it.

2. Based on this procedure of how the IEEE 1906.1 standard should be applied to an EM nano-communication development, we detected and documented a set of lacks that should be addressed in future versions.

3. Documentation of different solutions to better define both the framework and the metrics provided by the IEEE 1906.1 standard from the EM nano-communication perspective.

\section{$2.3 \quad$ Results and Contributions}

This paper brought to light different weaknesses of the IEEE 1906.1 standard that should be tackled to really achieve its objective. We divided them into four groups. The first one discussed the definition of the term nanoscale communication network, since it was excessively generic, while the three remaining groups reveal important debilities in each of the three lowest layers of the OSI reference model. Concretely, we detected the following weaknesses:

1. Excessively open definition of nanoscale communication network.

2. Lack of a reference energy model.

3. Signal-to-Noise Ratio (SNR) was not contemplated in the reference communication model.

4. Lack of layer 2 techniques to enhance communication robustness.

5. Equivalent layer 3 OSI reference model functions were not rigorously addressed.

6. Interconnections between a nanoscale communication network and macroscale devices were not addressed.

For each of them, we proposed the following open solutions:

1. More detailed definition of nanoscale EM communication devices. Two separate chapters for molecular and EM nano-communication are suggested.

2. Definition of a general enough energy model, but easily adapted to the technology employed. Energy restrictions should be better quantified to design a nanonetwork offering a real service.

3. Standardization of SNR values expected at reception to calculate appropriate receiver sensitivity thresholds. 
CHAPTER 2. THE IEEE 1906.1 STANDARD: NANOCOM. AS A NEW

SOURCE OF DATA

4. Recommendation of techniques referring to media access control, addressing scheme, flow control, and error detection.

5. Definition of routing procedures to allow multi-hop end-to-end communications. Design of a complete network topology.

6. Design and development of a link between the nano and macroscale worlds.

Therefore, our main contribution in this work was to identify and discuss the principal shortcomings of the IEEE 1906.1 standard and provide different guidelines to solve them.

\subsection{Conclusions}

The IEEE 1906.1 standard specified a set of recommended practices to enable researchers to jointly advance in the development of nano-communication systems. Even though it supposed an important step forward, we detected that more concreteness was necessary to envisage a common framework that became a solid foundation for designing forthcoming EM nanonetworks. Thus, in this work, we identified the most relevant shortcomings of the standard applied to EM nano-communication and offered possible guidelines to address each detected weakness in order to enhance the feasibility and potential of EM nano-communications. 



\section{Chapter 3}

\section{A nanoscale communication network scheme and energy model for a human hand scenario}

\subsection{Brief summary}

After more than one year working on EM nano-communications, we determined that the most promising application for EM nano-communications is in medicine, since nano-devices could be implanted into places where larger devices would be excessively intrusive. One of the potential scenarios was injecting nano-devices into the bloodstream so that they can flow through the human body monitoring different medical parameters such as body temperature, glucose levels, or even cancer biomarkers 20. However, nano-nodes (i.e. the smallest nano-device, which would be flowing through the circulatory system) are not able to directly communicate with external devices by their own. This is mainly due to the high absorption of EM waves at $\mathrm{THz}$ frequencies in aqueous mediums [29, which greatly reduces the communication range. In addition, the limited energy harvesting and storing capacity of nano-nodes should be taking into account to design a WNSN able to work into the human body.

In this context, no work in the related literature jointly considered all nanodevices limitations to design a realistic medical application employing WNSN. Therefore, in this work, we designed and developed a complete Body Area NanoNetwork (BANN), based on the knowledge acquired in our first work [1, able to monitor the human body. First, we designed a hierarchical BANN architecture composed of two types of nano-devices: nano-router and nano-node. Both nano-devices were conceived based on the features stated in [1, adapting the components of the nano-router to its specifications since it is significantly larger than a nano-node. Second, we developed a complete communication channel model at $1 \mathrm{THz}$ considering the biological tissues composing the path between nano-router and nano-nodes, that is, skin, fat, and blood. Finally, based on the nano-device specifications and the nano-nodes communication 
range, a straightforward communication scheme was proposed to allow the communication between nano-nodes and nano-router.

\subsection{Methodology and tools}

The methodology followed in this work was divided into four parts:

1. Analysis of the limitations of a WNSN deployment inside the human body. For that, exhaustive studies of both the EM propagation channel at $\mathrm{THz}$ frequencies into biological tissues and the energy harvesting inside the human body were carried out.

2. Design of the hierarchical BANN architecture to overcome the limitations analyzed and perform a useful application. In this phase, we also specify the features required by both nano-router and nano-nodes. We selected the dorsum of the hand as the scenario where the communication takes place to reduce the shortcomings derived from using $\mathrm{THz}$ frequencies and reduce the length of the nano-node/nano-router link.

3. Once the features of the nano-devices were specified, we conceptually designed both devices using technologically available components, based on the knowledge acquired in our first work.

4. Performance evaluation of the entire BANN, setting all the nano-network features (such as packet length, transmission power, nano-nodes charging time, and communication schedule), so that the entire BANN was technologically feasible.

\subsection{Results and Contributions}

Results revealed that each nano-node was able to send a medical measurement (inside a packet of $40 \mathrm{bits}$ ) to the nano-router every 52 min employing a hierarchical BANN with one nano-router implanted in the hand. The nano-router was designed to include a conventional communication module, allowing it to communicate with a wearable via $\mathrm{WiFi}$ or Bluetooth. In addition, the wearable, in this case a smart bracelet, was equipped with a small ultrasound source to provide a continuous energy source for the nano-router and notify nano-nodes that they are in the nano-router surroundings.

The contributions of this work can be listed as:

1. For the first time in the scientific literature, we provided a complete EM nano-communication network design able to monitor the human body, specifying all the features of the nano-devices composing the network.

2. We designed a specific energy model for a BANN considering and evaluating the limitations related to the biological tissues and the nanoscale. In addition, we developed a detailed study of radio propagation losses in the biological tissues of the human hand scenario under study 


\section{CHAPTER 3. A NANO COM. NETWORK SCHEME AND ENERGY \\ MODEL FOR A HUMAN HAND SCENARIO}

3. We conceived a communication scheme adapted to this BANN architecture that takes into account the energy limitations of nano-devices.

I would like to mention that this work has been very well accepted by the scientific community and is being considered as a reference work when talking about medical applications of nano-communications (it has been cited 30 times by the time of writing this thesis).

\subsection{Conclusions}

In this paper, we realistically designed a BANN architecture and communication scheme to allow the monitoring of the human body by overcoming the path loss and molecular absorption suffered in biological tissues. For that, we thoroughly studied both the EM channel and the energy balance to make the communication feasible employing realistic specifications. Even though this work was based on theoretical values, it provided an important step further in the feasibility of using nano-communications as a potential solution for medical applications. 



\section{Chapter 4}

\section{On the Nature of Energy-Feasible Wireless Nanosensor Networks}

\subsection{Brief summary}

In our previous work [3], we highlighted the importance of the energy balance in nano-devices, being one of the main limitations of a nano-network performance. Since the capacitor integrated into a nano-device is too small to be manually recharged or replaced, a piezoelectric generator is required to gather energy from the environment and provide a continuous source of energy. However, based on the harvesting energy values presented in [1] and 30], a nano-sized piezoelectric generator is not able to keep a nano-device always active, so they alternate between idle and active cycles. In order to optimize the period of these cycles for each scenario and application, an advanced model was needed to capture the impact of the different factors on the energy balance of a WNSN since this topic was not thoroughly studied in the related literature. Motivated by this lack in the literature, in this paper, we addressed the most important factors affecting both the energy harvesting and consumption of nanodevices, highlighting the effect of the communication scheme employed. The factors considered in the model were:

- Pulse transmission power

- Network topology

- EM path loss

- Packet length

- Generator and capacitor area

- Generator and capacitor technologies 
- Energy source

- Strain frequency

Based on all these parameters, we derived different mathematical expressions to measure the impact of each of them. Employing these expressions, we also evaluate the functionality of a nano-generator to determine the conditions under which a WNSN is viable from the energy point of view.

\subsection{Methodology and tools}

The methodology followed in this paper was geared towards obtaining a generic model to be employed in different WNSN developments for different scenarios and nano-devices. Therefore, most of the time was devoted to studying all the possible factors impacting on the energy balance in WNSN. To this effect, most of the works related to EM nano-communications were reviewed to analyze how the energy management of nano-devices was addressed. So, the steps followed are listed as follows:

1. Documentation and study of most of the published papers about EM nano-communications, focusing on how the energy harvesting and consumption of nano-devices were treated. Special attention was paid to the energy devoted to communication, since the values employed in the related literature were very disparate.

2. Documentation and study of how different factors, such as the generator and capacitor area or the technology employed, affect the energy harvesting process.

3. Analysis of the harvesting process in WNSN and derivation of mathematical expressions to model it considering all the parameters under study.

4. Validation of the mathematical expressions derived by comparing the results obtained in different works in the literature with those obtained with our model.

5. Development of a case of study based on the most typical values employed in the literature, delimiting the conditions under which the WNSN is feasible from the energetic point of view.

\subsection{Results and Contributions}

The results obtained in this paper can be divided into two sets:

1. Regarding the optimization of the energy harvesting system, results revealed that an adequate dimensioning of both energy harvesting (piezoelectric generator) and storage (capacitor) systems to the specifications of the communication scheme can significantly increase the bitrate achieved. 
CHAPTER 4. ON THE NATURE OF ENERGY-FEASIBLE WIRELESS

This is derived from the fact that an excessively large capacitor for a communication scheme with a reduced power consumption results in excessively long charging times. However, by adapting the capacitor capacity to the energy consumption requirements, the charging time can be optimized.

2. Regarding the energy feasibility of a WNSN, results showed that a piezoelectric micro-generator becomes inoperative for transmission distances over $1.5 \mathrm{~mm}$ in high-lossy environments (exceeding $100 \mathrm{~dB} / \mathrm{mm}$ ), as happens inside the human body. This is derived from the inability to harvest enough energy to feed the communication module in this scenario.

In relation to the results obtained, the contributions of this work can be listed as:

1. We synthesized the most important factors impacting on the energy consumption and harvesting in WNSN. To this end, we reviewed the most relevant works about WNSN in the scientific literature to illustrate the energy balance problem in nano-communications.

2. We provided a set of mathematical expressions that bring together all the parameters impacting on powering nano-system performance. These expressions constitute an excellent tool for optimizing the energy balance in future WNSN designs.

3. We performed a study of the viability of WNSN from the energy point of view, leading to relevant conclusions about the required characteristics that a EM-based WNSN must accomplish to be technically feasible.

To sum up, this work provided an excellent reference for all about energy management in WNSN and it is been really useful for future works during this thesis.

\subsection{Conclusions}

From this work, we extracted three main conclusions. First, we revealed that many published works about EM nano-communications assumed excessively high transmission powers, thus making the proposed nano-communication techniques energetically unfeasible. Second, the bitrate in WNSN can be increased by an appropriate dimensioning of the powering system. Finally, we conclude that for medical applications working inside the human body, $\mathrm{THz}$ communication links over $1.5 \mathrm{~mm}$ should be avoided to keep the viability of WNSN in terms of energy harvesting and consumption. 



\section{Chapter 5}

\section{The IEEE 1906.1 standard: Some guidelines for strengthening future normalization in electromagnetic nanocommunications}

\subsection{Brief summary}

This paper was conceived as an extended and more complete version of our previous conference paper 2, due to the positive feedback received by the scientific community. Thus, in this work, we presented a thorough review of the IEEE 1906.1 standard 8 from the electromagnetic (EM) nano-communications point of view, the field of study of this thesis. More specifically, we analyzed its definition of nano-communications, highlighting the pros and cons based on the know-how acquired in our previous papers, and discussed the proposed standardized framework with all its components. For that, we matched each framework component with its counterpart in an EM nano-communication network. Based on this application case of study about EM nano-communications, we identified open issues and challenges that should be addressed in future versions of the IEEE 1906.1 standard to meet the objective for which it was designed. As we were aware that the standard was still in an early stage, we did not provide specific solutions for specific problems, since each solution would require a research process before standardization. Thus, we pointed out the way to proceed for six relevant topics related to EM nano-communications, namely: (i) device architecture, (ii) energy harvesting and storage, (iii) energy consumption, (iv) SNR and channel characterization, (v) data link communication techniques, and (vi) routing techniques. 


\subsection{Methodology and tools}

The methodology of this work was similar to that presented in 2, that is:

1. In-depth analysis of the IEEE 1906.1 standard and envisage how it can be applied to (i) the design of generic nano-communication protocols and (ii) an EM nano-communication network case of study.

2. Based on the lacks detected in the previous step, we listed a set of points that should be more thoroughly addressed in future versions of the standard. For each point discussed, we investigated the literature to observe the big picture and detect how the standard should evolve to really meet its objectives.

3. Documentation of potential standard gaps and possible guidelines for addressing them to enhance the feasibility and capabilities of EM nanoscale communications based on the IEEE 1906.1 standard.

\subsection{Results and Contributions}

This paper revealed a set of lacks detected in the IEEE 1906.1 standard for EM nano-communications that should be filled in future versions. For these lacks, we provided different guidelines and recommended steps to take. Thus, contributions can be grouped into the six topics studied:

\section{Device architecture}

Detected lack: Main characteristics of a potential nano-device for EM nano-networks have not yet been addressed by the standard. It would be suitable to join standardization efforts to encourage a faster development of nano-communications.

Recommendation: More detailed definition of nanoscale EM communication devices. Two separate chapters for molecular and EM nanocommunication were suggested.

2. Energy harvesting and storage

Detected lack: The current version of the standard does not include a reference powering solution for nano-devices, which is of crucial importance to establish realistic values for energy harvesting and consumption.

Recommendation: Definition of a general powering solution for nanodevices. Energy restrictions in both harvesting and consumption should be better quantified to design a nano-network offering a real service.

3. Energy consumption

Detected lack: The standard would benefit from specifying recommended values for power transmission because it would better determine the 
CHAPTER 5. THE IEEE 1906.1 STANDARD: SOME GUIDELINES FOR

FUTURE NORMALIZATION IN EM NANOCOM.

transmission range of nano-nodes, the energy required to send information, and ensure that the power employed is not harmful to human health and the environment.

Recommendation: Standardization of power transmission within a defined range to amend the enormous variation between values employed in the literature.

4. SNR and channel characterization

Detected lack: Together with the standardization of power transmission, an SNR recommended range at reception would help future researchers to validate the coverage of nano-devices upon a common standard.

Recommendation: Standardization of an SNR value range expected at reception to calculate appropriate receiver sensitivity thresholds.

5. Data link communication techniques

Detected lack: The inclusion of data-link (layer 2) communication techniques basic core adapted to the characteristics of nano-networks, along with a standardized packet, would facilitate protocol interoperability.

Recommendation: Standardization of fundamental techniques referring to media access control, addressing scheme, flow control, and error detection.

6. Routing techniques

Detected lacks: Equivalent layer 3 OSI reference model functions should be addressed to improve the interoperability of routing techniques among nano-networks. The standard does not include any recommendation about the interconnection between a nanoscale communication network and macroscale devices, which could impact on other communication aspects.

Recommendations: Description of recommended routing procedures to allow multi-hop, end-to-end communications. Providing some recommendations or best practices about the design and development of a link between the nano and macroscale domains.

\subsection{Conclusions}

Even though the standard was aimed at providing a generic framework to facilitate researchers working in the same direction, its excessively permissive requirements could keep it away from its objectives. Based on our study, we concluded that researchers might not be motivated to change their methodology and designs to adapt them to the standard, since most of the prior and divergent works in EM nano-communications would comply with it. Therefore, we required more concreteness in future versions to make it effective. Having this in mind, we identified and discussed diverse gaps and offered possible 
CHAPTER 5. THE IEEE 1906.1 STANDARD: SOME GUIDELINES FOR 148 FUTURE NORMALIZATION IN EM NANOCOM.

guidelines for addressing each one to enhance the feasibility and capabilities of EM nanoscale communications. We also highlight the importance of dedicating efforts toward the standardization of the issues analyzed to adapt the EM communication features to the peculiarities of nano-devices.

Finally, we also detected that a cause of this excessive generalization could be derived from employing the same standard for molecular and EM nanocommunications. Thus, we proposed the division of the standard into two branches, one focused on EM nano-communications and the other for molecular nano-communications which should lead to a more suitable standardization for each type of nano-communication. 


\section{Chapter 6}

\section{Optimal Transmission Policy Derivation for IoNT Flow-Guided Nano-Sensor Networks}

\subsection{Brief summary}

Following the intention of improving the performance and viability of WNSN applied to medical applications, we designed a novel type of nano-network architecture coined as flow-guided nano-network (FGNN). This nano-network is characterized by the movement of nano-nodes through a well-defined stream of liquid, which applied to medicine would be the bloodstream. Together with this feature, FGNN includes a nano-router infrastructure along the stream, enabling the interconnection between nano-nodes and the Internet. Even though we conceived a nano-network architecture based on a similar paradigm in [3], we strictly defined the term flow-guided nano-network in this paper for the first time, specifying its characteristics and proposing a novel communication scheme tailored to this architecture.

In pursuit of achieving the maximum performance, we benefit from the experience of the research group in the IoT and applied a technique that was being recently employed in WSN, known as optimal transmission policies 34, to FGNN. To this end, we capture the benefits of this technique and adapt them to the particular features of nano-devices and FGNN. Concretely, the use of optimal transmission policies allowed nano-nodes to manage its scarce resources intelligently by determining whether or not it was beneficial to store, transmit or drop generated events as a function of its current state (i.e. battery level, distance to the router, or relevance of the sensed event, among others).

Therefore, in this paper, we designed an FGNN able to provide real-time measurements of medical parameters in vivo together with a generic mathematical framework based on Markov Decision Process (MDP) to derive optimal 
transmission policies for FGNN. We conceived this framework to be flexible enough to be applied in a wide range of applications in which FGNN could be employed. Transmission policies derived from our proposed model were validated by a set of simulations and compared with other alternative policies.

\subsection{Methodology and tools}

The first step in this methodology was to mathematically adapt and describe the different possible nano-nodes states in an FGNN, so that it could be modeled as a three-dimensional Markovian model. Even though there are plenty of parameters affecting the state of a nano-node, we determined that the three parameters composing each one of the three dimensions of the Markovian model were: (i) distance to the last traversed nano-router, (ii) battery level, and (iii) type of event generated. In turn, these three main parameters were affected by a set of secondary parameters, such as (i) duration of each time slot, (ii) nano-node velocity, (iii) energy harvesting rate, (iv) power consumption (that varies as a function of the action taken), (v) maximum battery capacity, (vi) packet length, (vii) maximum distance between routers, or (viii) coverage range. Therefore, we had to accurately determine the values of the three main parameters in the MDP considering all these secondary parameters. To this effect, we developed a detailed case of study taking realistic values found in the literature for each parameter, which is extensively explained in the published article [6. In addition, as Markovian models work with discrete values, where all magnitudes must be discretized, we had to determine how accurate was the discretization of all parameters mentioned above. Thus, we had to find a balance between accuracy and number of states, since the larger the number of states in the MDP the greater the accuracy defining the current state of the nano-node, but the higher the memory required to store it (which is quite limited in a nano-node). For that, we took as a reference the memory capacity determined in [1] and designed an MDP that could be stored in a nano-node with those features. Once the Markovian model was designed, we employed an efficient MDP algorithm (Q-learning) to determine the best action to take in each state to maximize a predefined reward. The reward maximized in this case was the prioritized throughput of nano-nodes (measured in bits per second), that is, the number of bits successfully transmitted per second by each nano-node to the nano-router infrastructure.

The final step was to simulate the FGNN under study employing SimPy 35, a discrete-event simulator, considering that the nano-nodes flowed through a closed-loop medium (simulating the cardiovascular system). Nano-nodes generate an event with a certain probability in each time slot, that depends on the scenario under study. There are two types of events generated with different priorities and lengths, detailed in the paper. The number of nano-nodes employed in the simulation was 6000. The policy derived from the MDP framework proposed was compared with five alternative intuitive transmission policies, also suitable for nano-nodes. All policies (including the MDP-based proposed one) 
CHAPTER 6. OPTIMAL TRANSMISSION POLICY DERIVATION FOR IONT FGNN

were evaluated under different scenarios (varying both the nano-routers location and the number of them) with 20 different seeds to obtain reliable results. It should be noted that the Python code employed for both the MDP model and FGNN simulations were programmed to be reusable and adaptable to researchers' requirements, being publicly available in 36 .

As the nano-router infrastructure cannot be considered perfectly deployed, we evaluate the impact of the precision on placing nano-routers. To this end, we defined the distance between nano-routers as $D_{N R}=U\left[D_{N R}^{*}-\delta, D_{N R}^{*}+\delta\right]$, so that $D_{N R}^{*}$ was fixed to $5 \mathrm{~cm}$ and $\delta$ was varied from $0 \mathrm{~cm}$ to $3 \mathrm{~cm}$. In other words, we assumed that the objective was to place a nano-router every $5 \mathrm{~cm}$, but it was done with different degrees of precision, modeled by $\delta$. For the second analysis, $\delta$ was set to $1 \mathrm{~cm}$ and $D_{N R}^{*}$ varied from $1 \mathrm{~cm}$ to $10 \mathrm{~cm}$ to analyze the effect of the total number of deployed nano-routers, since its linear density changes. Finally, we also evaluated three different event-generation rates scenarios to determine the benefits of the proposed policy as a function of the number of events generated. For that, the distance between nano-routers was set to $D_{N R}=5 \mathrm{~cm}$ with $\delta$ equal to 0 .

\subsection{Results and Contributions}

Results revealed that our policy systematically outperformed the other policies under all evaluated scenarios, even if the nano-router location was highly inaccurate. For the first set of simulations (varying the imprecision in placing nano-routers), the performance of the alternative policies heavily depends on the scenario. For example, in a high event-generation rate scenario OSHP (Only Store High-Priority events) policy shows the second-best performance whereas it falls to the fourth position for a low event-generation rate However, the MDP-based policy always performs the best, showing a greater difference over the alternative policies as the number of events generated becomes higher.

For the second set of simulations, the impact of the density of deployed nano-routers on the network prioritized throughput is investigated. By varying this parameter, the average distance between nano-routers is also modified, thus nano-nodes out-of-coverage periods may increase or decrease. Results obtained show that the throughput increases as the number of nano-routers becomes greater. In this case, the proposed MDP-policy performs better in the three event-generation scenarios, whereas the best alternative policy varies in each scenario.

Finally, for the third set of simulations, the prioritized throughput is obtained as a function of the probability of generating events per time slot. Results showed that the performance gap between the MDP-based and the second-best policy is greater as the probability of generating an event increases. This is derived from the fact that when the event-generation rate is quite low, the energy harvested by nano-nodes is large enough to handle the storage and transmission of all generated events. Therefore, simple alternative policies that store (and later transmit) every generated event tend to perform relatively well. 
However, when energy available is limited (either because the energy harvesting rate is reduced or the number of generated events increases, as is this case), smart policies such as the proposed one perform significantly better.

Regarding the main contributions of this paper, they can be summarized in the following four points:

1. A mathematical framework based on Markov decision processes (MDPs) for the computation of smart transmission policies for flow-guided nanonetworks was developed. The framework was conceived to be flexible enough to be applied in different applications and scenarios where flowguided nano-networks could be used.

2. A realistic case of study for the monitoring of the human circulatory system was thoroughly developed to apply the mathematical framework proposed. A detailed analysis of the communication link budget, energy harvesting, and power consumption rates was performed.

3. We provided a complete set of simulations of the derived smart policies in a wide range of scenarios and compare it to other alternative transmission policies to validate the analytical model and highlight its strengths.

4. We released all the programmed Python scripts to let researchers easily use or adapt the examples provided to their own needs and allow the reproducibility of results if desired.

\subsection{Conclusions}

In this paper, we proposed a general yet powerful mathematical framework that can assist designers in deriving smart transmission policies for flow-guided nano-nanonetworks. Relying on the results obtained from the simulation of a scenario based on the human cardiovascular system, we can conclude that our method effectively adapts the nano-node behavior to the particular characteristics of each environment, optimizing the scarce resource available and maximizing the performance. Compared to alternative transmission policies, the proposed policy requires from 35.6 to $150 \%$ fewer nano-routers to achieve similar performance. By reducing the number of deployed nano-routers we make the nano-network infrastructure less invasive and easier to implement, widening the scope and applicability of flow-guided nano-networks. 


\section{Chapter 7}

\section{An Analytical Approach to Flow-Guided Nanocommunication Networks}

\section{$7.1 \quad$ Brief summary}

The nano-network architecture presented in papers 3 and $[6$, in which nanonodes continuously move through a well-defined flow (coined as flow-guided nano-networks), was positively embraced by the scientific community due to its potential to be used in medical applications. By the time those papers were published, this was the first nano-network topology considering mobile nano-nodes, so its characteristics were rather different from other nano-network proposed in the related literature. As our previous papers were focused on specific scenarios and only measuring one metric (i.e. network throughput for a specific number of nano-nodes), there was a lack of generic mathematical models for flow-guided nano-networks that allow the scientific community to evaluate and predict its performance without the need for simulations.

Therefore, in this paper, we presented an extensive analytical framework to mathematically model the behavior of flow-guided nano-networks and evaluate different metrics as a function of diverse parameters considering collisions between nano-node transmissions. With this objective in mind, we derived a set of closed-form expressions for the maximum achievable throughput and collision rate as a function of the number of nano-nodes in the network, coverage range of nano-routers, battery recharge frequency, nano-node average velocity, and the number of transmission slots. Those expressions allowed us to obtain an optimal number of nano-nodes with its corresponding maximum throughput for each flow-guided nano-network and set theoretical boundaries to its performance.

In this context, any work in the related literature jointly considered all nano-devices limitations yet to design a realistic medical application employing WNSN. Therefore, in this work, we designed and developed a complete 
Body Area NanoNetwork (BANN), based on the knowledge acquired in our first work [1], able to monitor the human body. First, we designed a hierarchical BANN architecture composed of two types of nano-devices: nano-router and nano-node. Both nano-devices were conceived based on the features extracted in [1], adapting the components of the nano-router to its specifications since it is significantly larger than a nano-node. Second, we developed a complete communication channel model at $1 \mathrm{THz}$ considering the biological tissues composing the path between nano-router and nano-nodes, that is, skin, fat, and blood. Finally, based on the nano-device specifications and the nano-nodes communication range, a straightforward communication scheme is proposed to allow the communication between nano-nodes and nano-router.

\subsection{Methodology and tools}

The methodology employed in this work is divided into three main stages:

1. Analysis of the behavior of a flow-guided nano-network considering all the potential factors affecting its performance in terms of throughput, latency, and nano-node availability. The list of discussed parameters is as follows:

- Number of nano-nodes in the network

- Average speed of the nano-node in the flow

- Time required by a nano-node to complete a round in the flowguided network

- Nano-node charging frequency

- Nano-node communication range

- Channel path loss

- Length of the nano-router coverage area

- Length of the transmission zone

- Length of the collision zone

- Radius of the vein where the nano-router is placed

- Number of transmission slots between two battery charges

- Frame length

- Transmission bitrate

- Time required to transmit a complete frame

2. Development of an analytical model to characterize the behavior of a flowguided nano-network including all the parameters discussed in the first step. Frame collisions between nano-node frame transmissions (employing On-Off Keying modulation) and a time-slotted transmission technique 
were also considered in this model. Based on this analytical model, we derived different expressions to predict the theoretical achievable throughput and characterize the impact of: (i) using time-slotted transmission techniques, (ii) length of the coverage area, and (iii) frame length.

3. The final step was to validate the proposed mathematical model by simulating a flow-guided nano-network under different scenarios. Simulations were performed in Matlab considering these features:

- Nano-nodes are randomly deployed in a closed-loop system.

- Only a single transmission within the coverage of the nano-router was a successful frame transmission. No frames were received by the nano-router otherwise.

- Two or more simultaneous transmissions within the coverage area always collide.

- Nano-nodes could only send a frame between two battery charges.

- Nano-nodes randomly choose a transmission slot at every transmission cycle.

- Nano-nodes move at a constant average speed through the flow.

The simulation scenario variables were:

- Number of nano-nodes

- Number of transmission slots

- Length of the coverage area

- Battery charging frequency

- Nano-node speed in the flow

- Time to complete a round through the flow

- Simulation seed

Every point in the simulations was repeated ten times with a different seed. The results obtained with these simulations were compared to those resulting from the derived analytical expressions.

\subsection{Results and Contributions}

The results obtained can be grouped into three different sets as a function of the parameter under study, that is, (i) number of time slots, (ii) coverage area, and (iii) frame length. 


\section{Number of time slots}

The impact of the number of slots assigned on the nano-network throughput was noticeable when the number of nano-nodes was optimal to get the maximum throughput. In this case, a greater number of time slots resulted in an increment of the achievable throughput. Beyond the optimal point in which the throughput is maximal, a larger number of nano-nodes without increasing the number of slots would decrease the nano-network throughput. Likewise, if the number of nano-nodes is not enough to reach the maximum throughput (i.e. the probability of collisions between nano-nodes is still low), the impact of raising the number of slots would not have a remarkable impact.

\section{Coverage area}

Results revealed that the impact of the length of the coverage area on the maximum achievable throughput was not significant. However, by increasing the coverage area the number of nano-nodes required to achieve the maximum throughput is decreased. Thus, we can conclude that by designing the nanonetwork infrastructure to get larger lengths of the coverage area, fewer nanonodes are required to get the maximum performance, which can be beneficial for medical applications. Another interesting conclusion derived from these results is that to configure a flow-guided nano-network with a given lifetime (considering that some nano-nodes will eventually fail), a larger-than-optimal number of nano-nodes can be employed but reducing their coverage area (e.g. reducing the transmission power) to keep the nano-network throughput at the maximum. As the number of available nano-nodes becomes lower, the transmission power could be increased to keep the throughput near to the maximum during all the network lifetime.

\section{Frame length}

Regarding the frame length, results showed that a longer frame reduces nanonetwork throughput due to the growth of the area where collisions may occur, thus reducing the effective transmission area and increasing the probability of collisions. This effect is more significant when the coverage zone is small (around $0.5 \mathrm{~mm}$ ) whereas it becomes almost negligible for longer coverage zones.

The most important contributions of this paper are listed as follows:

1. We developed an analytical model capturing all the particularities of flowguided nano-networks. This model was general enough to be adapted to the requirements of different nano-networks.

2. We provided an extensive set of results regarding the achievable throughput of flow-guided nano-networks as a function of different relevant parameters. 
3. We calculated the optimal number of nano-nodes in a flow-guided nanonetwork that maximizes its throughput for diverse scenarios.

4. We proposed a novel time-slotted transmission technique and studied its positive impact on increasing the achievable throughput of flow-guided nano-networks.

\subsection{Conclusions}

In this paper, we proposed a novel theoretical framework to predict and evaluate the performance of flow-guided nano-networks. Results revealed that the model accurately predicts the nano-network performance obtained by the simulations in all cases, thus showing that it is a suitable method to estimate the performance of a flow-guided nano-network without the need for timeconsuming simulations.

Regarding the nano-network throughput, we concluded that there was a limit for the maximum achievable throughput that varies with the parameters studied, that is, coverage area, frame length, and number of time slots. This limit could be reached by using an optimal number of nano-nodes in the network, which can be employed for future flow-guided nano-network designs. 



\section{Part IV}

Conclusions and Future Lines 



\section{Chapter 1}

\section{Conclusions}

Electromagnetic nano-communications are emerging as a groundbreaking technology expected to bring light to a wide number of unexpected applications. The ability of nano-devices communicating at $\mathrm{THz}$ frequencies to be integrated into physical media where larger artificial devices would be excessively invasive can be a game changer in diverse application fields, with special interest in medicine. This is the reason why, even though a functional nano-device is not still available, there is an emerging research field to foresee the huge applicability of this paradigm and encourage its manufacturing.

In this context, this thesis has been focused on contributing to EM nanocommunications in the $\mathrm{THz}$ band from five different planes: (i) hardware, proposing a technologically feasible nano-device; (ii) communication, developing novel communication techniques and architectures for THz-based nanocommunications; (iii) energy, improving the energy consumption and harvesting models to adapt them to realistic conditions; (iv) optimization, proposing smart communication policies that can be implemented on nano-devices to optimize the resource utilization; and (v) standardization, proposing different recommendations for future versions of the IEEE 1906.1 standard.

In this thesis, we have presented seven different articles that successfully achieve the objectives pursued during this Ph.D. program. We humbly believe that these publications have resulted in a clear step further in the EM nano-communication field, proving a more realistic perspective of how nanocommunications should be envisioned. Even though each article shows its own conclusions, we synthesize them in the following paragraphs:

- Our first work provided the scientific community with a solid nano-device platform upon which EM nano-communication protocols could be designed. This aimed at solving the problem of employing excessively disparate values in the literature in terms of energy consumption, battery size, available memory, and processing capacity.

- Although EM nano-communications in the $\mathrm{THz}$ band are still in an early stage, more standardization efforts are required to really join efforts and 
promote future advances in nano-communications. Thus, an adequate standard could allow the development of interoperable and reusable components, protocols, and communication techniques.

- Realistic estimations of the path loss at $\mathrm{THz}$ frequencies must be taken into account for the design of nano-communication protocols and techniques, since both the energy required or the length of communication links can totally vary from the original estimation. These variations could make the protocol useless for most real-world applications.

- Even though the absorption of EM waves in the THz band when traveling through biological tissues is extremely high, useful applications can be developed if employing an adequate nano-network topology that reduces the length of communication links between nano-devices.

- In biological environments like the human body, there is a pressing need for having an accurate propagation model based on updated measurements. All estimations made during this thesis (as well as in published articles in the $\mathrm{THz}$ propagation field) have been performed based on data taken 20 years ago, so better characterizations could be attained nowadays with state-of-the-art equipment.

- More efforts devoted to developing a functional nano-powering system are required to make more accurate predictions at the time of design nano-communication protocols. As the envisioned energy harvesting and storing capacity of nano-devices is extremely limited, a small variation between the predicted (based on theoretical models that work for larger piezoelectric generators) and real energy harvested may lead to the communication protocol being ineffective. As an example of this, in [4], we proved that some communication schemes for EM nano-communications proposed in the literature do not comply with the threshold values identified in our energy analysis, so their energy viability in most scenarios is highly compromised.

- Even though experimental energy harvesting and storing values for EM nano-devices are not available yet, in this thesis, we have contributed with a detailed energy harvesting and consumption analysis assuming the values of each parameter as realistic as possible. We also revealed that the bitrate of a WNSN can be optimized for a specific application by properly designing the power generation system. It represents a great tool for optimizing the energy balance in future EM nano-network designs.

- As a way to optimize the scarce resources of nano-devices, we have demonstrated in this thesis that smart transmission policies can be implemented in nano-nodes (i.e. the smallest device composing an EM nano-network). If the scenario can be appropriately modeled, these policies can be derived offline in regular computers (since the computation capacity required is high) and then installed and used by nano-nodes without the need for 
additional processing tasks. The Markov Decision Process framework proposed for flow-guided nano-networks should be flexible enough to be applied in a wide range of applications.

- Due to the short action range of nano-devices, the number of required nano-nodes in most WNSN is expected to be quite high. This leads to high time-consuming simulations, thus hindering the optimization of different parameters in a nano-network. Therefore, analytical models able to predict the performance of an EM nano-network are of paramount importance to speed up the optimization process. Trying to fill this gap, we have proposed an analytical framework to model the behavior of a flow-guided nano-network and make an extensive analysis of the impact of different parameters on its performance.

In essence, the above conclusions highlight the main areas this thesis has been focused on. Contributions made throughout this thesis have been geared towards making EM nano-communications in the $\mathrm{THz}$ band one step closer to becoming a reality. 



\section{Chapter 2}

\section{Future Lines}

Even though some critical issues that demand research efforts from the scientific community have been already pointed out in the previous section, we outline here some points in which we plan to work in the future.

At the time of writing this thesis, there is one more article under review in one of the most relevant journals in the field. This work delves into:

1. Design a novel THz-based flow-guided nano-network architecture aimed at monitoring both general medical parameters and critical parts of the human body. For that, a medical device located in specific internal parts of the body sensing a vital sign of interest has been conceived. It is equipped with a communication module able to transmit the acquired information to mobile nano-nodes that act as information carriers.

2. Development of an analytical model capturing all the special characteristics of this novel nano-network architecture. An approach based on Markov chains has been employed for that.

3. Analysis of the performance of potential flow-guided nano-networks to be used in different medical applications, providing the specifications of each EM nano-network to meet the application requirements.

We would like to highlight that this work was conducted in cooperation with medical personnel, so all the results rely on realistic values of the cardiovascular system and the medical applications discussed have been approved by them.

Apart from this work, even though the improvement of the presented EM nano-networks and nano-communication techniques and models will still be the foundation of future works, we also plan to follow these three main research lines:

1. Implement a more detailed simulator based on the real flow of the cardiovascular system to get more accurate and closer to reality simulation values. This simulator could also be an excellent platform to propose and test novel EM nano-communication protocols for in-body nano-networks. 
The first steps in this direction have already been taken in cooperation with the development team of BitSimulator 37,38, one of the most promising simulators for EM nano-communications.

2. Develop a more realistic and accurate characterization of the $\mathrm{THz}$ band absorption in biological tissues employing state-of-the-art equipment. The research stay at University of California in Davis (UCDavis) is aimed at this research line.

3. Work on the design of novel $\mathrm{THz}$ nano-generation sources easily embeddable into a micro circuit board. Promising results have been already obtained by the research group at UCDavis but further work is required. 


\section{Part V}

Appendices 



\title{
Impact factor of publications
}

\author{
(Source: JCR Science Edition and Google Scholar)
}

\section{Article 1}

Title: "Conceptual design of a nano-networking device"

Journal and Publisher: Sensors, MDPI

Date of acceptance: 6 December 2016

Impact Factor: 2.677

Quartile: Q1 (Instrument \& Instrumentation)

Cited by: 20

\section{Article 2}

Title: "A nanoscale communication network scheme and energy model for a human hand scenario"

Journal and Publisher: Nano Communication Networks, Elsevier

Date of acceptance: 22 January 2018

Impact Factor: 2.250

Quartile: Q2 (Telecommunications) y Q2 (Engineering, Electrical \& Electronic)

Cited by: 30

\section{Article 3}

Title: "On the Nature of Energy-Feasible Wireless Nanosensor Networks"

Journal and Publisher: Sensors, MDPI

Date of acceptance: 24 April 2018

Impact Factor: 3.031

Quartile: Q1 (Instrument \& Instrumentation)

Cited by: 12

\section{Article 4}

Title: "The IEEE 1906.1 standard: Some guidelines for strengthening future normalization in electromagnetic nanocommunications"

Journal and Publisher: IEEE Communications Standards Magazine, IEEE Date of acceptance: 4 June 2018

Impact Factor: -

Quartile: -

Cited by: 4

Article 5

Title: "Optimal Transmission Policy Derivation for IoNT Flow-Guided Nano- 
Sensor Networks"

Journal and Publisher: Internet of Things Journal, IEEE

Date of acceptance: 12 March 2019

Impact Factor: 9.936

Quartile: Q1 (Telecommunications), Q1 (Computer Science, Information Systems) y Q1 (Engineering, Electrical \& Electronic)

Cited by: 5

\section{Article 6}

Title: "An Analytical Approach to Flow-Guided Nanocommunication Networks"

Journal and Publisher: Sensors, MDPI

Date of acceptance: 25 February 2020

Impact Factor: 3.275

Quartile: Q1 (Instrument \& Instrumentation)

Cited by: 1 


\section{Part VI}

\section{References}





\section{Bibliography}

[1] S. Canovas-Carrasco, A.-J. Garcia-Sanchez, F. Garcia-Sanchez, and J. Garcia-Haro, "Conceptual Design of a Nano-Networking Device," Sensors, vol. 16, no. 12, p. 2104, Dec. 2016.

[2] S. Canovas-Carrasco, A.-J. Garcia-Sanchez, and J. Garcia-Haro, "The IEEE 1906.1 standard: Nanocommunications as a new source of data," in 2017 ITU Kaleidoscope: Challenges for a Data-Driven Society (ITU K), 2017, pp. 1-7.

[3] S. Canovas-Carrasco, A.-J. Garcia-Sanchez, and J. Garcia-Haro, "A nanoscale communication network scheme and energy model for a human hand scenario," Nano Communication Networks, vol. 15, pp. 17-27, Mar. 2018.

[4] S. Canovas-Carrasco, A.-J. Garcia-Sanchez, and J. Garcia-Haro, "On the Nature of Energy-Feasible Wireless Nanosensor Networks," Sensors, vol. 18, no. 5, p. 1356, Apr. 2018.

[5] S. Canovas-Carrasco, A.-J. Garcia-Sanchez, and J. Garcia-Haro, "The IEEE 1906.1 Standard: Some Guidelines for Strengthening Future Normalization in Electromagnetic Nanocommunications," IEEE Communications Standards Magazine, vol. 2, no. 4, pp. 26-32, Dec. 2018.

[6] S. Canovas-Carrasco, R. M. Sandoval, A. Garcia-Sanchez, and J. GarciaHaro, "Optimal Transmission Policy Derivation for IoNT Flow-Guided Nano-Sensor Networks," IEEE Internet of Things Journal, vol. 6, no. 2, pp. 2288-2298, 2019.

[7] R. Asorey-Cacheda, S. Canovas-Carrasco, A.-J. Garcia-Sanchez, and J. Garcia-Haro, "An Analytical Approach to Flow-Guided Nanocommunication Networks," Sensors, vol. 20, no. 5, p. 1332, Feb. 2020.

[8] "IEEE Recommended Practice for Nanoscale and Molecular Communication Framework," IEEE Std 1906.1-2015, pp. 1-64, 2016.

[9] I. Akyildiz and J. Jornet, "The Internet of nano-things," IEEE Wireless Communications, vol. 17, no. 6, pp. 58-63, Dec. 2010. 
[10] J. M. Jornet and I. F. Akyildiz, "The internet of multimedia NanoThings," Nano Communication Networks, vol. 3, no. 4, pp. 242-251, Dec. 2012.

[11] S. Balasubramaniam and J. Kangasharju, "Realizing the internet of nano things: Challenges, solutions, and applications," Computer, vol. 46, no. 2, pp. 62-68, 2013.

[12] A. D. Franklin, "Nanomaterials in transistors: From high-performance to thin-film applications," Science, vol. 349, no. 6249, 2015. [Online]. Available: https://science.sciencemag.org/content/349/6249/aab2750

[13] I. Llatser, C. Kremers, A. Cabellos-Aparicio, J. M. Jornet, E. Alarcón, and D. N. Chigrin, "Graphene-based nano-patch antenna for terahertz radiation," Photonics and Nanostructures - Fundamentals and Applications, vol. 10, no. 4, pp. 353 - 358, 2012, taCoNa-Photonics 2011.

[14] I. Park, Z. Li, A. P. Pisano, and R. S. Williams, "Top-down fabricated silicon nanowire sensors for real-time chemical detection," Nanotechnology, vol. 21, no. 1, p. 015501, nov 2009.

[15] N. Shehada, G. Brönstrup, K. Funka, S. Christiansen, M. Leja, and H. Haick, "Ultrasensitive silicon nanowire for real-world gas sensing: Noninvasive diagnosis of cancer from breath volatolome," Nano Letters, vol. 15, no. 2, pp. 1288-1295, 2015.

[16] Y. Ke, T. Meyer, W. Shih, and G. Bellot, "Regulation at a distance of biomolecular interactions using a DNA origami nanoactuator," Nature Communications, vol. 7, p. 10935, 032016.

[17] C. Xing, Y. Huang, J. Dai, L. Zhong, H. Wang, Y. Lin, J. Li, C.-H. Lu, and H.-H. Yang, "Spatial Regulation of Biomolecular Interactions with a Switchable Trident-Shaped DNA Nanoactuator," ACS Applied Materials E Interfaces, vol. 10, no. 38, pp. 32 579-32 587, 2018.

[18] Y. Chahibi, "Molecular communication for drug delivery systems: A survey," Nano Communication Networks, vol. 11, pp. 90-102, Mar. 2017.

[19] U. A. K. Chude-Okonkwo, R. Malekian, B. T. Maharaj, and A. V. Vasilakos, "Molecular communication and nanonetwork for targeted drug delivery: A survey," IEEE Communications Surveys \& Tutorials, vol. 19, no. 4, pp. 3046-3096, 2017.

[20] P. M. Kosaka, V. Pini, J. J. Ruz Martínez, R. A. d. Silva, M. U. González Sagardoy, D. Ramos Vega, M. Calleja, and F. J. Tamayo de Miguel, "Detection of cancer biomarkers in serum using a hybrid mechanical and optoplasmonic nanosensor," Nature Nanotechnology, vol. 9, no. 12, pp. 1047-1053, Nov. 2014. 
[21] P. Kulakowski, K. Solarczyk, and K. Wojcik, "Routing in fret-based nanonetworks," IEEE Communications Magazine, vol. 55, no. 9, pp. 218$224,2017$.

[22] L. Felicetti, M. Femminella, G. Reali, and P. Liò, "Applications of molecular communications to medicine: A survey," Nano Communication Networks, vol. 7, pp. 27-45, Mar. 2016.

[23] J. M. Jornet and I. F. Akyildiz, "Graphene-based plasmonic nano-antenna for terahertz band communication in nanonetworks," IEEE Journal on Selected Areas in Communications, vol. 31, no. 12, pp. 685-694, 2013.

[24] A. Cabellos-Aparicio, I. Llatser, E. Alarcón, A. Hsu, and T. Palacios, "Use of terahertz photoconductive sources to characterize tunable graphene rf plasmonic antennas," IEEE Transactions on Nanotechnology, vol. 14, no. 2 , pp. 390-396, 2015.

[25] K. Sengupta, T. Nagatsuma, and D. Mittleman, "Terahertz integrated electronic and hybrid electronic-photonic systems," Nature Electronics, vol. 1, pp. 622-635, 2018.

[26] J. M. Jornet and I. F. Akyildiz, "Graphene-based plasmonic nanotransceiver for terahertz band communication," in The 8th European Conference on Antennas and Propagation (EuCAP 2014), 2014, pp. 492-496.

[27] F. Lemic, S. Abadal, W. Tavernier, P. Stroobant, D. Colle, E. Alarcón, J. Marquez-Barja, and J. Famaey, "Survey on terahertz nanocommunication and networking: A top-down perspective," 2019.

[28] I. F. Akyildiz and J. M. Jornet, "Electromagnetic wireless nanosensor networks," Nano Communication Networks, vol. 1, no. 1, pp. 3-19, Mar. 2010.

[29] K. Yang, A. Pellegrini, M. O. Munoz, A. Brizzi, A. Alomainy, and Y. Hao, "Numerical Analysis and Characterization of $\mathrm{THz}$ Propagation Channel for Body-Centric Nano-Communications," IEEE Transactions on Terahertz Science and Technology, vol. 5, no. 3, pp. 419-426, May 2015.

[30] J. M. Jornet and I. F. Akyildiz, "Joint Energy Harvesting and Communication Analysis for Perpetual Wireless Nanosensor Networks in the Terahertz Band," IEEE Transactions on Nanotechnology, vol. 11, no. 3, pp. 570-580, May 2012.

[31] S. Abadal, C. Liaskos, A. Tsioliaridou, S. Ioannidis, A. Pitsillides, J. SoléPareta, E. Alarcón, and A. Cabellos-Aparicio, "Computing and communications for the software-defined metamaterial paradigm: A context analysis," IEEE Access, vol. 5, pp. 6225-6235, 2017. 
[32] S. Abadal, A. Mestres, J. Torrellas, E. Alarcon, and A. Cabellos-Aparicio, "Medium Access Control in Wireless Network-on-Chip: A Context Analysis," IEEE Communications Magazine, vol. 56, no. 6, pp. 172-178, June 2018.

[33] A. Afsharinejad, A. Davy, B. Jennings, and C. Brennan, "Performance Analysis of Plant Monitoring Nanosensor Networks at THz Frequencies," IEEE Internet of Things Journal, vol. 3, no. 1, pp. 59-69, 2016.

[34] R. M. Sandoval, A.-J. Garcia-Sanchez, J. Garcia-Haro, and T. M. Chen, "Optimal Policy Derivation for Transmission Duty-Cycle Constrained LPWAN," IEEE Internet of Things Journal, vol. 5, no. 4, pp. 3114-3125, Aug. 2018.

[35] Simpy, "Event discrete simulation for Python." [Online]. Available: https://simpy.readthedocs.io

[36] R. M. Sandoval and S. Canovas-Carrasco, "Python scripts for simulations," 2019. [Online]. Available: https://github.com/rubmarsan/ IoTjournal-IEEE-2019

[37] D. Dhoutaut, T. Arrabal, and E. Dedu, "Bit simulator, an electromagnetic nanonetworks simulator," in Proceedings of the 5th ACM International Conference on Nanoscale Computing and Communication, ser. NANOCOM '18, 2018.

[38] D. Dhoutaut, T. Arrabal, and E. Dedu, "BitSimulator, a C++ wireless nanonetwork simulator for routing and transport levels," 2019. [Online]. Available: http://eugen.dedu.free.fr/bitsimulator/ 


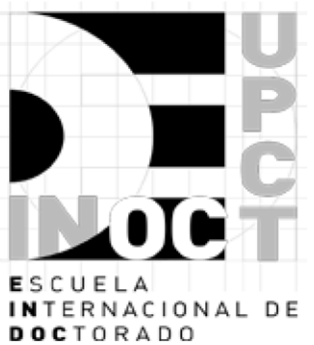

